\title{
Selective Attention and Perceptual Load in Autism Spectrum Disorder
}

\section{Anna Remington}

University College London

A thesis submitted for the degree of Doctor of Philosophy

September 2009 


\section{Declaration}

I, Anna Remington confirm that the work presented in this thesis is my own. Where information has been derived from other sources, I confirm that this has been indicated in the thesis.

A Remington 


\section{Abstract}

This thesis examines selective attention in young adults with Autism Spectrum Disorder (ASD). Existing literature regarding this issue is mixed; some research suggesting an overly-focused attentional style (Rincover \& Ducharme, 1987) while others highlight an abnormally broad attentional lens (Burack, 1994).

The research presented here has, for the first time, examined selective attention in individuals with ASD using a theoretically-led approach based on Lavie's Load Theory of attention and cognitive control (Lavie et al., 2004). Load theory states that the perceptual load (amount of potentially task relevant information) of a task affects selective attention. This theory may explain the equivocal findings in the current data on selective attention and ASD.

Using behavioural measures, the pattern of selective attention under various levels of load was explored in individuals with ASD and matched controls. The results provide evidence of increased perceptual capacity in ASD. This means that, at any one time, individuals with ASD may be able to process more information from the visual environment. This increase in capacity was evident on tasks of both unconscious and conscious perception.

In light of the social deficits observed in the condition, the work in this thesis also explored selective attention in the presence of social distractor stimuli. Results indicated that faces are less salient for individuals with ASD and, unlike for typical adults, are not processed in an automatic and mandatory fashion. These results bring together findings on selective attention with work on social processing in an attempt to find basic abnormalities which might be fundamental in explaining the disorder. 
For Guy 


\section{Contents}

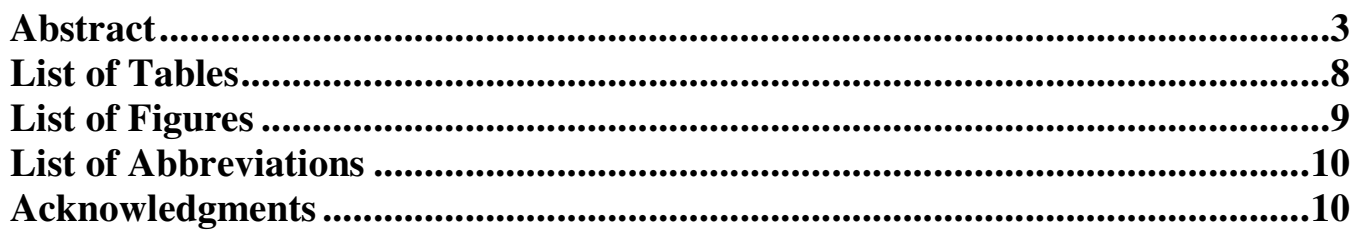

Chapter 1: Autism Spectrum Disorder ..........................................................12

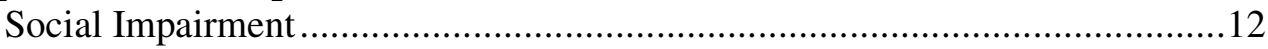

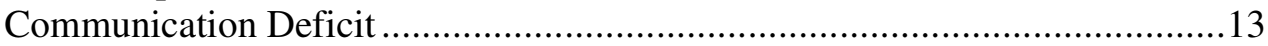

Repetitive and Rigid Behaviour............................................................13

Asperger Syndrome and Autism Spectrum Disorder..........................................14

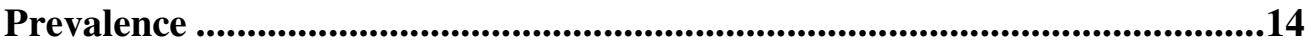

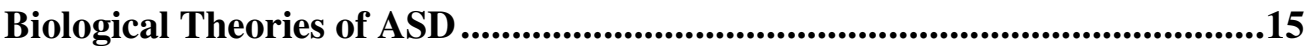

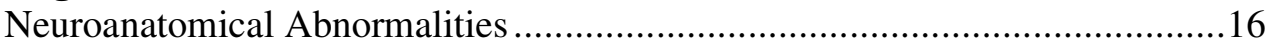

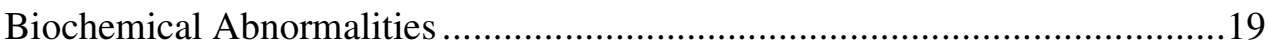

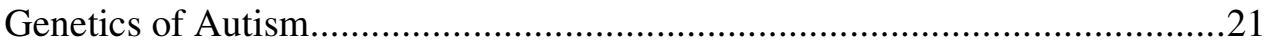

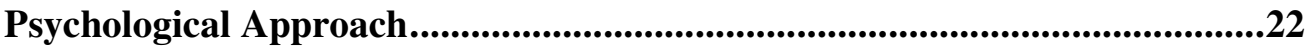

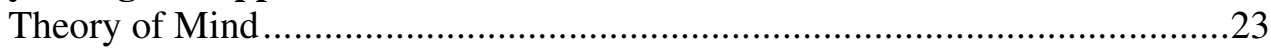

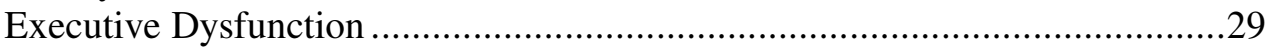

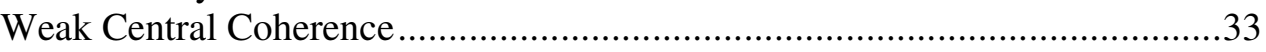

Systemizing, Empathizing and the Extreme Male Brain ...............................36

Summary .....................................................................................................37

Chapter 2: Selective Attention and Perceptual Load ..............................................40

Selective Attention ................................................................................................41

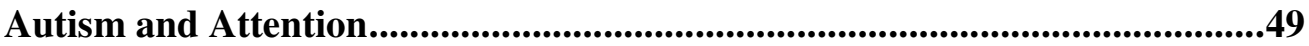

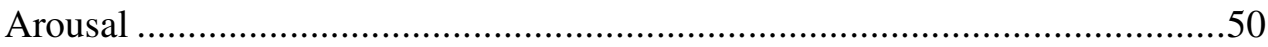

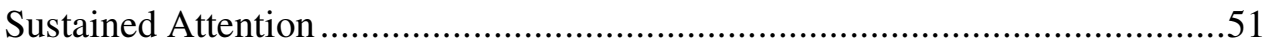

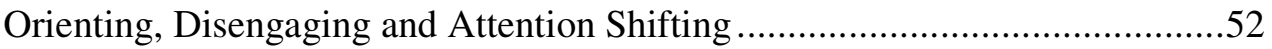

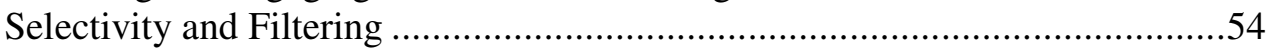

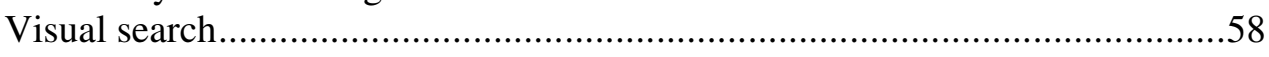

Chapter 3: Selective Attention and Perceptual Load in ASD ..........................62

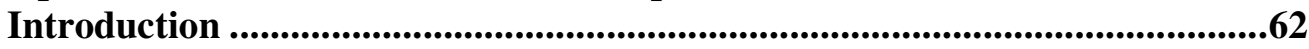

Participants and Diagnosis .........................................................................63

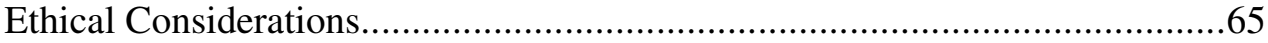

Methodology ...........................................................................................................65

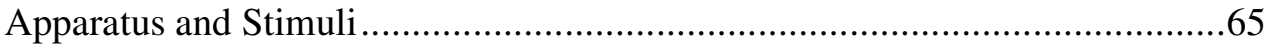

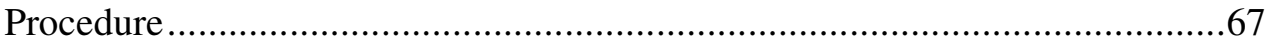

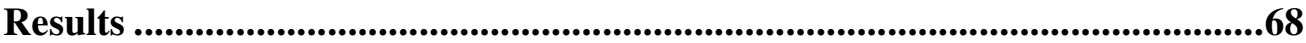

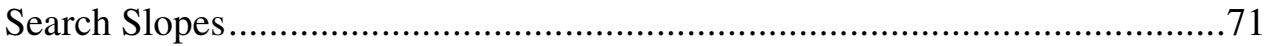

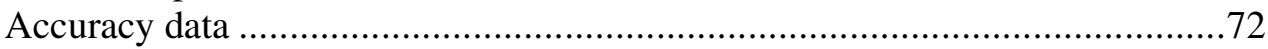

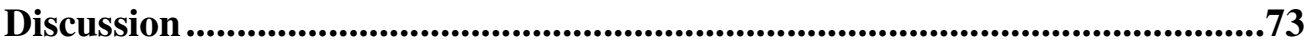

Chapter 4: Perceptual Load and Conscious Perception in ASD.........................76

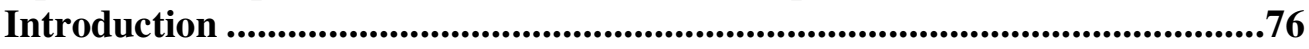


Participants .....................................................................................................................79

Methodology ....................................................................................................80

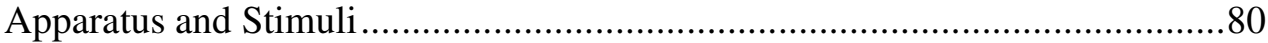

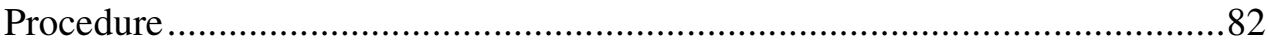

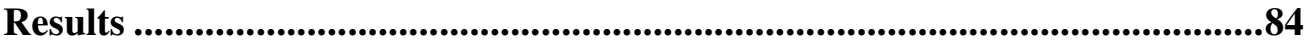

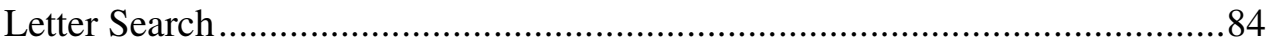

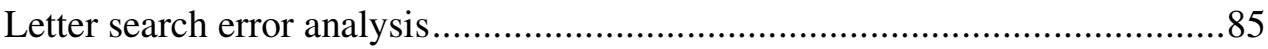

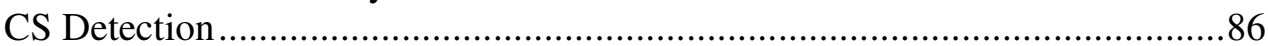

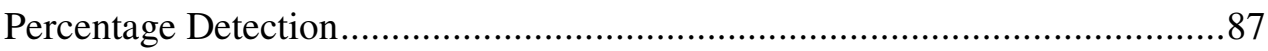

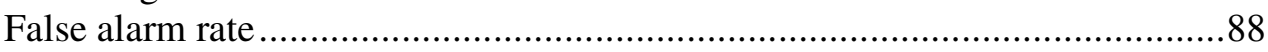

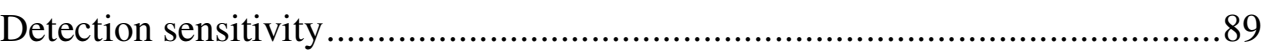

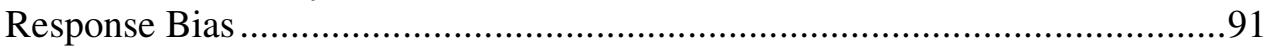

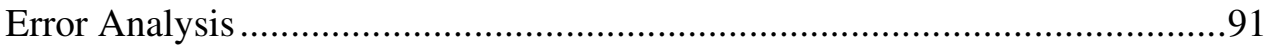

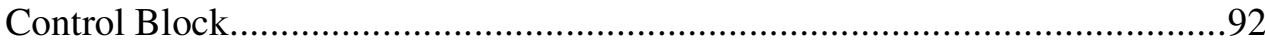

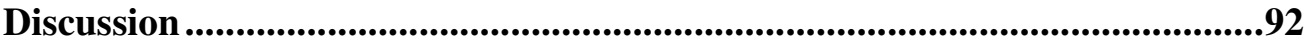

Mixed trial paradigm..............................................................................................99

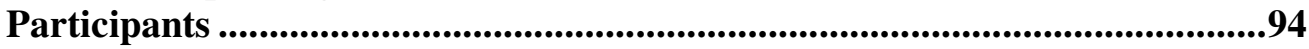

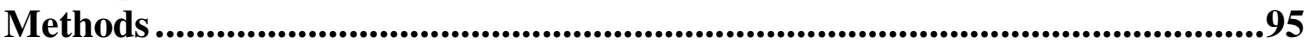

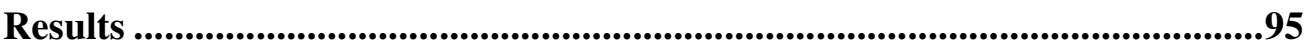

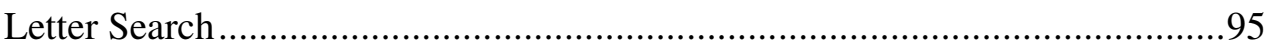

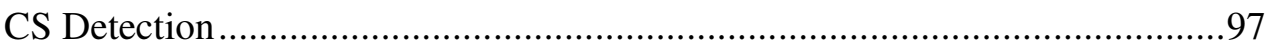

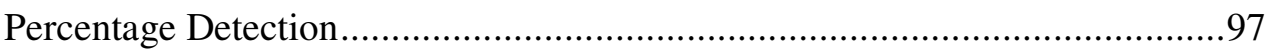

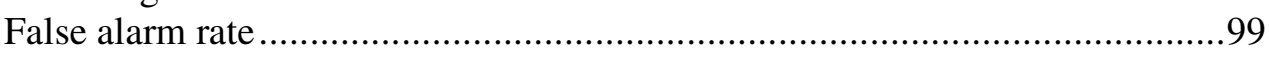

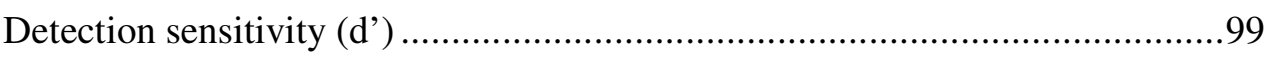

Discussion ....................................................................................................................102

Comparing the blocked and mixed trial procedures ......................................103

Comparison of search task RT data in experiment two and three ..................103

Comparison of RT error rates in experiment two and 3..............................103

Comparison of detection rates in experiment two and 3..............................104

Comparison of false alarm rates in experiment two and 3..........................105

Comparison of detection sensitivity in experiment two and 3....................105

Comparison of CS error rates in experiments two and three..........................106

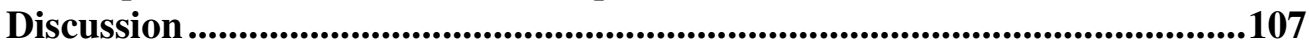

Overall Discussion..........................................................................................109

Chapter 5: Visual Short Term Memory Capacity in ASD ...............................112

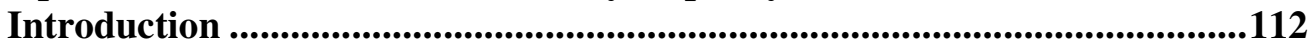

Visual Short-Term Memory ..................................................................113

Calculating visual short-term memory capacity .....................................116

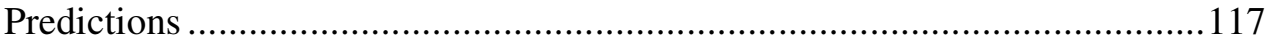

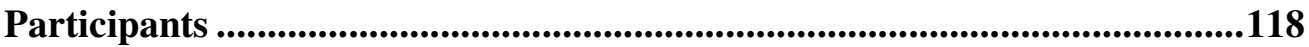

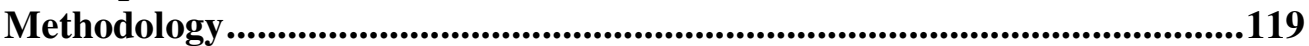

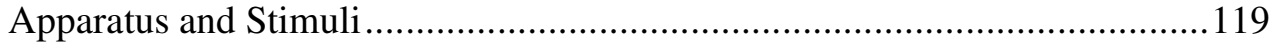

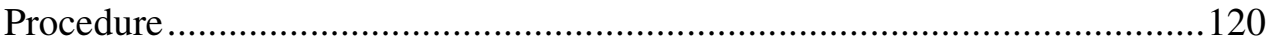

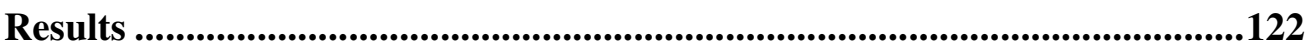

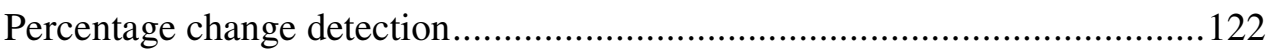

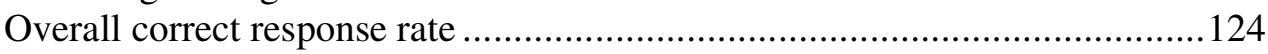

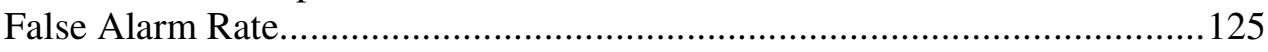

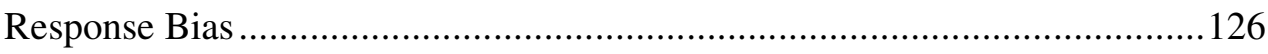

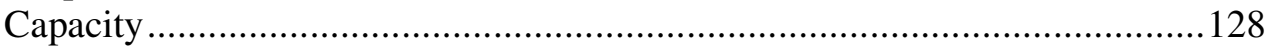


Discussion ................................................................................................................130

Chapter 6: Selective Attention and Perceptual Load with Face Distractors....135

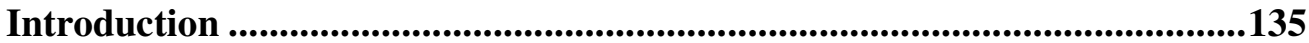

Face Processing in Typical Individuals ..................................................138

The Development of Face Processing Abilities..............................................138

Are Faces Special? .................................................................................... 140

Faces processing as a mandatory and automatic process ............................144

Face Processing in ASD ................................................................145

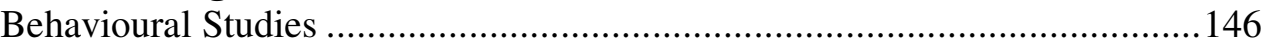

Neuroimaging and electrophysiological Studies ...................................... 147

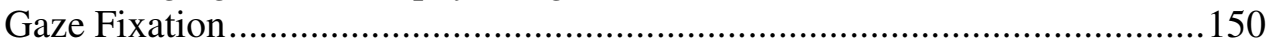

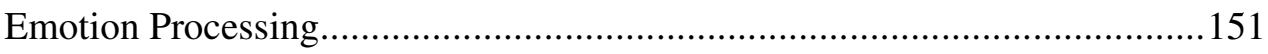

Aetiology of abnormal face processing in ASD ......................................152

Attention to social stimuli - are faces special in ASD? ................................154

Summary .......................................................................................................155

Effect of Non-Famous Faces as Distractors in Perceptual Load Tasks .........156

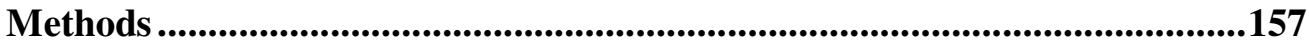

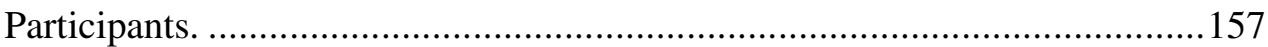

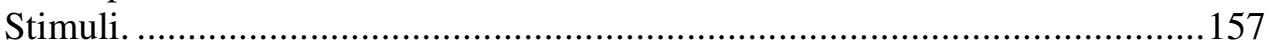

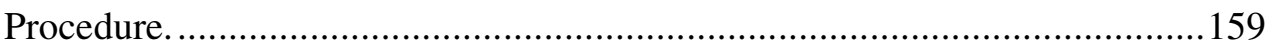

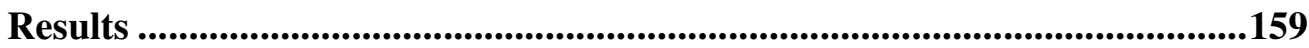

Discussion ...................................................................................................................161

Faces as distractor elements in a task of selective attention .........................162

with ASD. .......................................................................................................................162

Methods .................................................................................................................163

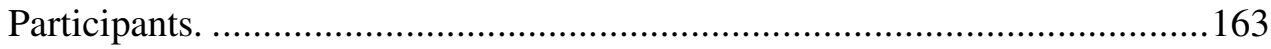

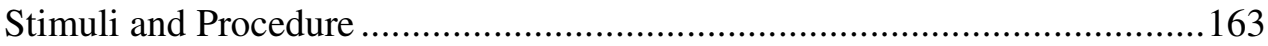

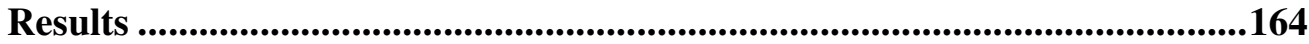

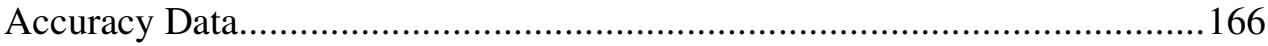

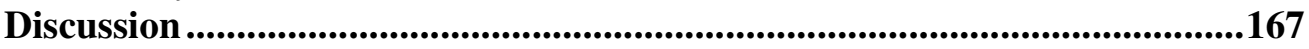

Musical Instruments as distractor elements .............................................167

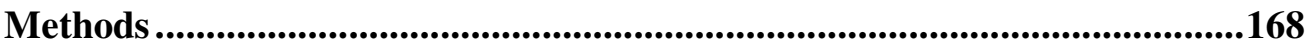

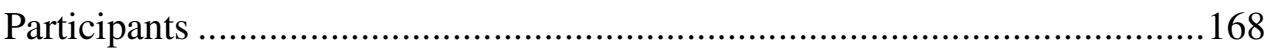

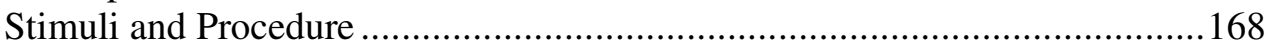

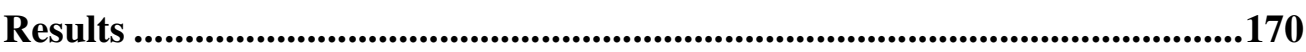

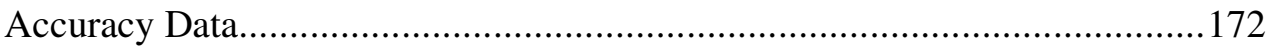

Comparing distractor interference from face and instrument distractors.........174

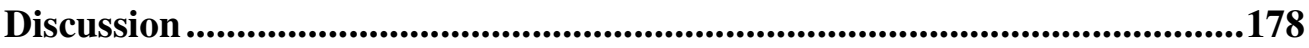

Overall Discussion............................................................................................................179

Chapter 7 - Discussion .....................................................................................183

Implications of increased perceptual capacity ...........................................191

Implications of reduced interference by social distractors ..........................201

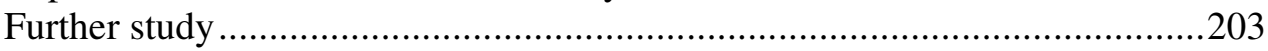

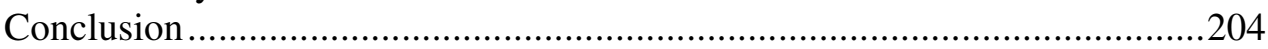

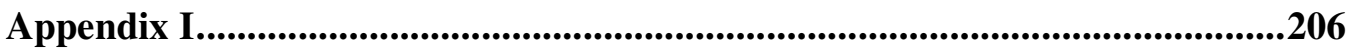

Appendix II ...........................................................................................206

Appendix III....................................................................................................................2069

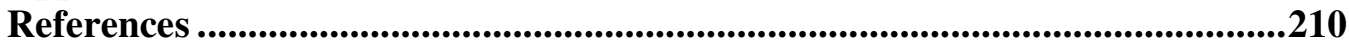




\section{List of Tables}

$\begin{array}{lll}\text { Table 3.1 Descriptive statistics for each group } & 64\end{array}$

Table 3.2 Overall mean response times and standard deviations 68

$\begin{array}{lll}\text { Table 3.3 Accuracy rates and standard deviations } & 72\end{array}$

$\begin{array}{lll}\text { Table 4.1 Descriptive statistics for each group } & 79\end{array}$

Table 4.2 Mean response times and standard deviation 85

Table 4.3 Mean error rates and standard deviations 86

Table 4.4 Mean CS detection rate, false alarm rate and d' 87

$\begin{array}{lll}\text { Table 4.5 } & \text { Mean response bias }(\beta) \text { for each group at each set size } & 91\end{array}$

Table 4.6 Mean error rates and standard deviation 92

Table 4.7 Mean response times and standard deviation 95

Table 4.8 Mean error rates and standard deviation 96

Table 4.9 Mean CS detection rate, false alarm rate and d' 97

$\begin{array}{lll}\text { Table 4.10 } & \text { Mean response bias }(\beta) \text { and standard deviation }\end{array}$

$\begin{array}{lll}\text { Table 4.11 Mean error and standard deviation } & 101\end{array}$

Table 5.1 Descriptive Statistics for each Group 119

Table 5.2 Mean hit rate and standard deviation 122

$\begin{array}{lll}\text { Table 5.3 Mean correct responses and standard deviation } & 124\end{array}$

$\begin{array}{lll}\text { Table 5.4 Mean false alarm rate and standard deviation } & 125\end{array}$

$\begin{array}{lll}\text { Table 5.5 } & \text { Mean response bias }(\beta) \text { and standard deviation } & 127\end{array}$

$\begin{array}{lll}\text { Table 5.6 } & \text { Mean visual short-term memory capacity } & 128\end{array}$

$\begin{array}{lll}\text { Table 6.1 Descriptive Statistics for participants } & 158\end{array}$

$\begin{array}{lll}\text { Table 6.2 Mean response times and standard deviations } & 160\end{array}$

$\begin{array}{lll}\text { Table 6.3 Descriptive Statistics for each group } & 164\end{array}$

$\begin{array}{lll}\text { Table 6.4 Mean response times and standard deviations } & 165\end{array}$

$\begin{array}{lll}\text { Table 6.5 Accuracy rates and standard deviations } & 167\end{array}$

$\begin{array}{lll}\text { Table 6.6 } & \text { Descriptive Statistics for each group } & 169\end{array}$

Table 6.7 Mean response times and standard deviations 172

$\begin{array}{lll}\text { Table 6.8 Accuracy rates and standard deviations } & 174\end{array}$ 


\section{List of Figures}

Figure 1.1 Example of the stimuli used within the Navon Task 35

Figure 2.1 Filter theory of early selection (Broadbent, 1958) 42

Figure 2.2 Late-selection theory of attention (Deutsch \& Deutsch, 1963) 43

Figure 2.3 Filter Attenuation Theory of Attention (Treisman, 1964) 44

Figure 2.4 Examples of experimental stimuli (Maylor \& Lavie, 1998) 47

Figure 3.1 Examples of stimuli for experiment one 66

$\begin{array}{lll}\text { Figure 3.2 Difference between response times } & 69\end{array}$

$\begin{array}{lll}\text { Figure 3.3 Mean response times at each set size } & 70\end{array}$

$\begin{array}{lll}\text { Figure 3.4 Response times for neutral trials at each set size } & 72\end{array}$

Figure 4.1 Examples of experimental stimuli for experiment two 81

$\begin{array}{lll}\text { Figure 4.2 Critical stimulus positions } & 81\end{array}$

$\begin{array}{lll}\text { Figure 4.3 Time-course of experimental trials } & 84\end{array}$

$\begin{array}{lll}\text { Figure 4.4 Critical stimulus detection rates } & 88\end{array}$

$\begin{array}{lll}\text { Figure 4.5 } & \text { False alarm rates. } & 89\end{array}$

$\begin{array}{lll}\text { Figure 4.6 Detection sensitivity } & 90\end{array}$

$\begin{array}{lll}\text { Figure 4.7 } & \text { Critical stimulus detection rates } & 98\end{array}$

Figure 4.8 False alarm rates of each group under each condition 99

$\begin{array}{lll}\text { Figure 4.9 } & \text { Detection sensitivity } & 100\end{array}$

$\begin{array}{lll}\text { Figure 5.1 Time-course of experimental trials } & 121\end{array}$

$\begin{array}{lll}\text { Figure 5.2 Mean percentage of changes detected } & 122\end{array}$

$\begin{array}{lll}\text { Figure 5.3 Mean correct response rates } & 124\end{array}$

$\begin{array}{lll}\text { Figure 5.4 Mean false alarm rates } & 126\end{array}$

$\begin{array}{lll}\text { Figure 5.5 Visual short-term memory capacity } & 128\end{array}$

Figure 6.1 Example of stimuli used by Lavie et al., $2003 \quad 137$

$\begin{array}{lll}\text { Figure 6.2 } & \text { Example of a chimeric face } & 140\end{array}$

Figure 6.3 Examples of stimuli used in experiment five 159

$\begin{array}{lll}\text { Figure 6.4 Time course of experimental trials } & 160\end{array}$

$\begin{array}{lll}\text { Figure 6.5 Median response times for each trial condition } & 161\end{array}$

Figure 6.6 Difference in response times (incongruent/congruent trials) 162

Figure 6.7 Congruency effects for each group at each set size 166

$\begin{array}{lll}\text { Figure 6.8 } & \text { Example of stimuli used in experiment seven } & 171\end{array}$

$\begin{array}{lll}\text { Figure 6.9 Congruency effects for each group at each set size } & 173\end{array}$

$\begin{array}{lll}\text { Figure 6.10 Congruency effects in ASD group } & 176\end{array}$

$\begin{array}{lll}\text { Figure 6.11 Congruency effects in control group } & 177\end{array}$ 


\section{List of Abbreviations}

\begin{tabular}{r|l} 
& \\
ADHD & Attention Deficit Hyperactivity Disorder \\
ADOS & Autism Diagnostic Observational Schedule \\
ANOVA & Analysis of variance \\
AS & Asperger's Syndrome \\
ASD & Autism Spectrum Disorder \\
CAST & Childhood Asperger Syndrome Test \\
CPT & Continuous performance tests \\
CS & Critical stimulus \\
DSM-IV & Diagnostic and statistical manual of mental disorders \\
DZ & Dizygotic \\
ED & Executive Dysfunction \\
EEG & Electroencephalography \\
EFT & Embedded Figures Task \\
EPF & Enhanced perceptual functioning \\
EQ & Empathising Quotient \\
ERP & Event related potential \\
FFA & Fusiform face area \\
fMRI & Functional magnetic resonance imagery \\
IFC & Inferior frontal cortex \\
ITG & Inferior temporal gyrus \\
MEG & Magnetoencephalography \\
MZ & Monozygotic \\
NART & National Adult Reading Test \\
OCD & Obsessive Compulsive Disorder \\
PET & Positron emission tomography \\
PM & Premotor Cortex \\
RT & Reaction time \\
SD & Standard deviation \\
SM & Systemising mechanism \\
SPECT & Single photon emission computed tomography \\
SSRI & Selective serotonin reuptake inhibitor \\
STS & Superior temporal sulcus \\
ToM & Theory of Mind \\
VSTM & Visual short term memory \\
WASI & Wechsler Abbreviated Scale of Intelligence \\
WCC & Weak Central Coherence \\
WCST & Wisconsin Card Sorting Test \\
Then &
\end{tabular}




\section{Acknowledgments}

The completion of this thesis is a testament to the amazing support I have received from those around me over the past three years. Firstly, I would like to thank my supervisor, John Swettenham, for all his help and advice - and for always treating me as a colleague rather than a student. I have so enjoyed our time working together and I hope this is the start of many future collaborations.

Thanks also go to Ruth Campbell for her guidance and encouragement, to Mike Coleman for his invaluable help with task programming, and to Nilli Lavie, whose work provided the starting point for this thesis, for her wise comments and constant enthusiasm for my research.

I would like to extend my thanks to the many members of the Department of Developmental Science at UCL who have helped along the way, and to the participants who gave up their time to take part in my studies - none of this would have been possible without you.

Special thanks go to my hilarious flatmate Schaff, to office-mate, Tanya Denmark for keeping me company and keeping me sane, to Ålex Pine for the countless study sessions and constant motivation and to my parents for inspiring me and for supporting my attempt to become Dr Remington the Third. Lastly, my thanks go to Guy for his unwavering support, for always sharing the burden and for putting up with 'thesis Anna'. This is for you. 


\section{Chapter 1: Autism Spectrum Disorder}

In the 1940s, Leo Kanner (1943) and Hans Asperger (1944) independently described a condition that they had observed where individuals show social impairments, repetitive behaviours and narrow obsessive interests. While subtle differences were present in their definitions, both embraced the idea of an autistic condition that could account for this symptom profile. Confirmation that this was indeed a syndrome rather than a number of co-morbid conditions was provided by Wing and Gould (1979). Their epidemiological study of children living in Camberwell, London demonstrated that all the children with social impairments also showed repetitive stereotyped behaviour and most of them had deficits in language and communication. This formed the basis for the triad of impairments that, to this day, has been consistently used to define autism (American Psychiatric Association, 1994). Each of these deficits will now be discussed.

\section{Social Impairment}

The difficulty with social interaction that is seen in autism typically revolves around creating and sustaining meaningful social relationships. Individuals with autism often fail to make friends and have problems engaging in reciprocal social interaction. They may avoid social contact altogether or may endeavour to interact with others in an inappropriate manner. It seems that autistic individuals have an inability to understand social norms and are incapable of interpreting the social cues that are vital for successful interaction. 


\section{Communication Deficit}

The second defining feature of the condition is a delay or, in severe cases, an absence of communicative ability. Language onset is generally late in development and there are often marked abnormalities in the speech intonation, rate and rhythm. Speech also often contains a degree of echolalia and stereotyped phrases that are repeated frequently. Non-verbal communication abilities are also affected. When displayed at all, eye contact and gesture are often poorly modulated. There is also a distinct lack of communication as a social tool. Language is used for purely functional reasons to produce a desired outcome rather than to connect with other individuals.

\section{Repetitive and Rigid Behaviour}

This third dimension to the triad describes the tendency to rigidly adhere to routines, to show stereotypical movements, such as hand flapping, and to have narrow obsessions and areas of interest. An individual with autism might, for example, follow a daily routine meticulously - never deviating from a route to school/work, eating the same food in the same order or running through the same set of behaviours each time they leave the house. A passionate obsession about a narrow field of interest, such as train timetables, dates on a calendar or television aerials is also characteristic of this third area of the triad.

While this triad is sufficient for the diagnosis of autism, there are a number of other features that are often noted within the condition. One such feature is that of abnormal attentional abilities which have been widely reported (see Burack et al., 
1997 for a review). It is upon this observation that the work of this thesis is based and therefore this issue will be fully discussed in a subsequent chapter.

\section{Asperger Syndrome and Autism Spectrum Disorder}

While closely related, it has been asserted that the two conditions that Kanner and Asperger described were not identical. Asperger's clinical population had a higher language ability than those described by Kanner and were generally less severely impaired. Although there is still a debate about the presence of Asperger Syndrome as a distinct condition, the term is currently used to define highfunctioning individuals who display social impairments and repetitive behaviour but who have no language delay (American Psychiatric Association, 1994).

It is now also accepted that the severity of autism can vary and some researchers such as Simon Baron-Cohen believe that it is one continuous spectrum of attributes that ranges through the population from typical individuals up to severely autistic individuals (Baron-Cohen \& Hammer, 1997). The term Autism Spectrum Disorder (ASD) is now commonly used to refer to all individuals that exhibit a level of autistic traits above the cut off for the condition as prescribed in DSM-IV.

\section{Prevalence}

Epidemiological studies of the condition since the year 2000 report a prevalence of between 7.2 and 40.5 cases of ASD per 10,000 individuals. However, a recent study carried out in the UK estimates the true value to be somewhat higher: with ASD affecting around $1 \%$ of child population (Baird et al., 2006). There is an 
uneven distribution between the sexes; with a male to female ratio of approximately 4:1(Fombonne, 2003; Fombonne, 2009).

\section{Biological Theories of ASD}

A great deal of research has attempted to identify the cause of this condition. The theories and observations can be divided broadly into biological and psychological research. These two areas will be discussed in turn.

Much research has pointed towards an underlying biological deficit that may be involved in ASD. This view is based on observations such as the fact that up to one third of individuals with autism go on to develop epilepsy before they reach adulthood (Gillberg \& Coleman, 1992; Steffenburg, 1990). Likewise, genetic conditions such as fragile $\mathrm{X}$ syndrome, tuberous sclerosis, phenylketonuria, Rett syndrome and neurofibromotosis occur at higher levels than in the general population (Blomquist et al., 1985; Cohen et al., 1991; Gillberg, 1983; Gillberg et al., 1984a; Gillberg et al., 1984b; Gillberg et al., 1985; Gillberg \& Wahlstrom, 1985; Reiss et al., 1986).

Neurological research has highlighted a number of brain regions that may be associated with the condition. The parietal lobes (Courchesne et al., 1993), prefrontal cortex (Prior \& Hoffman, 1990), medial temporal lobe structures (Salmond et al., 2005) and the cerebellum (Courchesne, 1997) have primarily been implicated. 


\section{Neuroanatomical Abnormalities}

\section{Cerebellar Dysfunction}

Using magnetic resonance imaging (MRI) Courchesne and colleagues found cerebellar hypoplasia in individuals with autism - and that the extent of the condition was not correlated with the severity of learning deficit (Courchesne et al., 1988). While this data and corroborating findings in post-mortem studies (Kemper \& Bauman, 1993) led to a cerebellar-based theory of autism, the MRI data has subsequently been difficult to replicate and PET studies have found no evidence of cerebellar hydroplasia (Heh et al., 1989). To date, the most consistent microscopic findings are the absence or reduction in number of the purkinje cells in the cerebral hemispheres (Hossein Fatemi et al., 2002) .

\section{Medial temporal lobe damage}

A body of evidence has also suggested that the medial temporal lobe structures are affected in autism. It was observed that temporal lobe damage resulting from herpes simplex encephalitis has been seen to cause severely autistic behaviour in previously typical adults (Gillberg, 1991). Similarly, autistic traits - both communicative and social - were seen in a boy with a left temporal oligodendroglioma (tumour) (Hoon, Jr. \& Reiss, 1992) and, as mentioned above, the prevalence of temporal lobe epilepsy (Gillberg \& Coleman, 1992) and tuberous sclerosis of temporal lobes (Bolton \& Griffiths, 1997) within the autistic population is much higher than within the general population. Autopsy studies have added further weight to this theory by demonstrating the presence of subtle 
neuropathological changes in the hippocampus, amygdala, septum and mamillary bodies (Kemper \& Bauman, 1993).

\section{Parietal Lobe Abnormalities}

MRI studies have shown that there are parietal abnormalities in over $30 \%$ of individuals with autism (Courchesne et al., 1993) and using Single-Photon Emission Computed Tomography (SPECT), reduced blood flow within the parietal region has also been detected (George et al., 1992). Furthermore, when performing neuropsychological tests of parietal functioning, individuals with autism score poorly irrespective of intelligence level (Haas et al., 1996).

\section{Prefrontal Cortex}

Results regarding the theory that prefrontal cortex deficiencies are evident in autism have been mixed. A handful of PET and MRI studies have indicated the presence of damage in this region (Gaffney et al., 1989; Hashimoto et al., 1989; Siegel, Jr. et al., 1992) but these findings have not been widely replicated. SPECT studies have highlighted a reduction in blood flow (George et al., 1992) which, it has been suggested, is the result of a delay in frontal cortex maturation in individuals with autism (Zilbovicius et al., 1995). More recent studies demonstrate that children with ASD have abnormally large frontal lobes (Carper \& Courchesne, 2000; 2005) which may reflect a lack of synaptogenesis in early life (Belmonte et al., 2004). 


\section{Macrocephaly}

Kanner mentioned in his original definition of the condition that five of the eleven children studied had unexpectedly large heads (Kanner, 1943). This observation has been raised a number of times since then. Bailey et al.(1995) showed that $42 \%$ of autistic twins below the age of 16 year in their study had a head circumference above the $97^{\text {th }}$ percentile, while Bolton et al. (1994) found that this was the case in $22 \%$ of 87 children and adults with autism. Imaging and post mortem studies have shown that this is due to increased brain volume (Bauman, 1996; Piven et al., 1995). The differing figures seem to be a reflection of the discrepancies within the definition of macrocephaly ( $>97^{\text {th }}$ or $>98^{\text {th }}$ percentile) and the incidence of microcephaly in low-functioning individuals with autism that is likely to be due to co-morbid medical disorders. Fombonne and colleagues examined 126 children in an attempt to clarify the levels of macrocephaly seen in autism. They found that one out of every six autistic subjects had macrocephaly (Fombonne et al., 1999). In an even larger collaborative study, 338 individuals with autism were studied (Lainhart et al., 2006). While the distribution of head circumference was seen to be normal within this sample, the mean circumference and rate of macrocephaly were raised in autism. In a recent longitudinal study, Webb and colleagues examined the rate of head circumference growth (extracted from medical records) of a group of boys with ASD and a group with developmental delay over the first three years of life (Webb et al., 2007). They found that there was a significantly higher rate of growth in the ASD group - more specifically, a statistically significant increase in occipitofrontal circumference between 7 and 10 months, relative to norms. It is currently unclear how this physical abnormality is related to the behavioural and neuroanatomical profile of autism. 


\section{Biochemical Abnormalities}

\section{Dopamine}

Damasio and Maurer (1978) compared their neuropsychological patients to individuals with autism and noted that deficits in the structure or functioning of the mesolimbic cortex and its related structures produced a similar symptom profile. Given that the target area of the ascending mesencephalic dopamine projection may therefore be implicated, it may be the case that a neurochemical abnormality which disrupts dopaminergic systems is responsible for the dysfunction. While there is no direct evidence for dopaminergic dysfunction in autism, the hypothesis has been supported up by a number of experimental studies. Abnormal dopamine metabolism has been suggested in autism, as seen by the high levels of the dopamine metabolite homovanillic acid in cerebrospinal fluid and urine (Gillberg \& Coleman, 1992). Furthermore, an increased blink rate in autism (an indication of dopamine hyperactivity) has been reported (Goldberg et al., 1987) and the dopamine antagonist, haloperidol, is used with some success in the treatment of autism (Anderson et al., 1984).

\section{Serotonin}

The neurotransmitter serotonin has also been implicated in autism. Increased platelet serotonin (hyperserotonemia) has been consistently found in about one third of individuals with autism (Cook \& Leventhal, 1996). Most individuals with autism who are given serotonin transporter inhibitors such as the antidepressant clomipramine, or selective serotonin reuptake inhibitors (SSRIs) such as fluoxetine, show a reduction in ritualistic behaviours and aggression. Furthermore, reduction of 
the central nervous system serotonin, caused by induced tryptophan depletion, caused a worsening of stereotyped behaviour (McDougle et al., 1996). Hyperserotonemia has been found to be familial - with a correlation between levels of parental and proband serotonin (Kuperman et al., 1985). It has also been noted that individuals with autism who had a sibling with autism showed higher platelet serotonin levels than other individuals with autism (Piven et al., 1991). Hyperserotonemia may therefore be a useful marker of autism that has a higher risk of sibling recurrence.

\section{Foetal Testosterone}

Baron-Cohen and colleagues have put forward an alternative biological theory concerning autism (Baron-Cohen et al., 2004). They suggest that higher than normal levels of pre-natal testosterone are responsible for the autistic condition. After noting that the classic autistic behaviours seem to be an extreme of the male brain (see later discussion on systemising and empathising), Baron-Cohen examined the levels of foetal testosterone in autistic individuals. This was done by following up a group of 100 children whose foetal testosterone had been measured through amniocentesis (Baron-Cohen et al., 2004). The amount of eye contact at 12 months of age was seen to vary quadratically with amniotic testosterone level, suggesting that foetal testosterone may affect social development. At 48 months, amniotic testosterone levels were seen to be negatively correlated with the quality of social interaction and positively correlated with restricted interests -even when sex differences were accounted for. Subsequently, an additional 400 children were added to the study and were followed up at age five and beyond using the Childhood Asperger Syndrome Test (CAST), the Empathising Quotient (EQ) and the 
Systemising Quotient (SQ). The results indicated that the number of autistic traits shown in children was related to foetal testosterone levels (Auyeung et al., 2008). It remains to be seen, however, whether variations in foetal testosterone relate to the severity of symptoms within individuals with ASD. As yet, only variations within the normal population have been examined.

\section{Genetics of Autism}

In the 1960s, 20 years after Kanner's original description, Rutter (1968) noted that approximately $2-3 \%$ of families in the early case studies had more than one autistic child. He highlighted that this was 50-100 times greater than that which would be predicted by population prevalence. Reinforced by the first demonstration that there was a higher concordance rate for autism among monozygotic (MZ) twins than among dizygotic (DZ) twins (Folstein \& Rutter, 1977), the idea of a genetic component to autism began to emerge. Twin studies have produced a wealth of information regarding the heritability of autism (Bailey et al., 1995; Folstein \& Rutter, 1977; Steffenburg et al., 1989). The results from the most recent of these three epidemiological twin studies (Bailey et al., 1995) indicated a MZ concordance rate of $60 \%$ and a DZ concordance rate of $0 \%$ for pure autism. When the analysis was broadened to include the wider ASD phenotype, the MZ concordance rate increased to $92 \%$ and the $\mathrm{DZ}$ rate to $10 \%$. Using these figures and the recurrence risk, the heritability estimate was calculated to be more than $90 \%$. While this result is striking, it may also be based on the fact that there was very little environmental variation within the populations that were studied. It is accepted, however, that there must be some sort of genetic influence on the condition. The nature of this influence is yet to be explicitly defined. Pickles et al. (1995) reject single locus and 
heterogeneity models and suggest a multilocus model with between two and ten loci and three epistatic (interacting) loci. A multi-gene model such as this is consistent with the observation that family members of an autistic proband may exhibit mild autistic-like phenotypes or Asperger syndrome. While the exact genes implicated differ between studies, the most consistent signals are on chromosome $7 \mathrm{q}$ and $15 \mathrm{q}$ (Gillberg, 1998; Scherer et al., 2003; Schroer et al., 1998). Given the uneven male:female sex ratio (4:1) in autism, the $\mathrm{X}$ chromosome has also been the focus of much research. Two genome-wide scans have recently found evidence of linkage to the X-chromosome but the findings are inconsistent (Liu et al., 2001; Shao et al., 2002). Furthermore, evidence of father-to-son transmission, examination of extended pedigrees and the broader phenotype suggest that X-linkage would only explain a small percentage of the genetic variance (Hallmayer et al., 1996).

As is evident from the information above, there are many suggestions regarding an organic basis for autism but there is no consistent and direct evidence for a particular area of neurobiological damage or dysfunction or a specific gene that may underlie the condition. It is possible that we are not yet able to accurately identify the crucial neurobiological substrate or it may be that autism is a complex condition resulting from a number of diverse aetiologies (Goodman, 1989).

\section{Psychological Approaches}

In response to the presence of a distinct behavioural profile and the absence of an accepted biological aetiology for autism, a great deal of research has been done to investigate whether a primary psychological deficit could be responsible. Three major theories have dominated the literature; Theory of Mind (ToM), Executive 
Dysfunction (ED) and Weak Central Coherence (WCC), and these will be discussed in turn before mentioning a more recent theory regarding autism as the extreme male brain.

\section{Theory of Mind}

Premack and Woodruff (1978) introduced the phrase 'theory of mind' to describe the capacity to ascribe mental states to oneself and others. It encompasses the ability to make appropriate inferences about the feelings, beliefs, desires and knowledge of others and therefore both understand and predict their actions. It has been argued that the possession of ToM is a major factor involved in meaningful social behaviour (Baron-Cohen et al., 1985) and the ToM hypothesis of autism proposes that an absence of ToM in individuals with autism is a fundamental deficit within the condition (Baron-Cohen et al., 1985; Frith, 1989; Leslie \& Frith, 1987; Leslie \& Happe, 1989). Wulff's observation that children with autism show an absence of early pretend-play fuelled this theory (Wulff, 1985). Leslie (Leslie \& Frith, 1987) argued that make-believe play that does not interfere with real world knowledge depends on two levels of representation: primary representation of objects and events, and metarepresentations that define people's propositional attitudes to those objects and events (i.e. ToM). He went on to suggest that individuals with autism have difficulty forming metarepresentations and therefore would not be able to successfully perform tasks that require ToM (Baron-Cohen et al., 1985; Frith, 1989; Leslie \& Frith, 1987; Leslie \& Happe, 1989).

ToM is classically tested by using a false belief task. This is where participants are asked to predict the behaviour of another individual who holds a false belief. Baron-Cohen et al.(1985) devised the Sally-Anne task to assess 
understanding of false belief. In this task, the experimenter tells the child about a doll, Sally, who puts her marble into a basket and then leaves the room. While Sally is out of the room, a second doll, Anne, moves the marble into a box. The story is acted out with dolls and props to help the child follow the events and a series of control questions are asked to check that the child has followed the events. The child is then asked to say where Sally will look for the marble when she comes back into the room. If the child understands that Sally has a false belief that differs from the real state of the world then s/he will indicate Sally will look in the original location (the basket). Such a response neatly demonstrates that the child understands that Sally's mental state (the belief that the marble is in the basket) differs from their own (the belief that the marble is in the box). Wimmer \& Perner (1983) showed that typically developing children of three to four years old tend to fail the test. From about four to five years old, children begin to pass this test and indicate that Sally will look in the original location. By the age of six years old, the children consistently understood that another person can hold a false belief about the world.

Baron-Cohen et al. (1985) tested twenty children with autism with a verbal mental age of over four years on the Sally-Anne task. $80 \%$ of the children failed the task - something that could not simply be attributed to general mental handicap as $86 \%$ of a control group of children with Down's Syndrome passed the test, even though they had a lower mean verbal mental age than the group with autism. The deficit therefore seemed to be specific to autism and not developmental delay. This finding has been subsequently replicated a number of times, and has also been found when using alternative ToM tasks such as 'The Smarties Test' (Perner et al., 1989).

As is evident from Baron-Cohen et al's results above, some children with ASD do manage to pass the ToM test. It was shown, however, that the majority 
of these children fail to pass more sophisticated versions of the false belief task (Baron-Cohen, 1989). This study used a more difficult test of second order false belief understanding created by Perner and Wimmer (1985) that involves secondorder belief (understanding what one person thinks about another person's belief). The extra level of embedding increases the complexity of the task and typically developing children generally pass the test only after the age of 7 years. BaronCohen demonstrated that children with autism with a mental age above seven years failed this second order task - even many of those who had managed to pass the first order task (Baron-Cohen, 1989). Happé further examined the subgroup of children with ASD that seemed to be able to pass false belief tasks. She created the Strange Stories Test, a more naturalistic test of ToM. Within this task, participants are presented with stories about situations where people do not say literally what they mean (e.g. telling someone that their new dress is nice when you believe it is ugly). Using this more contextually embedded paradigm, Happé required the children to determine whether a statement made by a character in the story was true or false, and to comment on why the character said the statement. The results showed that even children with autism who passed both first and second order false-belief tasks were impaired on the Strange Stories Test (Happe, 1994).

The question is, therefore, whether the ToM hypothesis can explain the behavioural profile of autism. The theory definitely seems consistent with the social deficits observed. Young children with autism are seen to treat other people as objects (Baron-Cohen, 1990), and often show an interest in other children but cannot appropriately interact with them (Lord, 1993). They also appear to successfully understand simple emotions which do not require the decoding of mental states (happiness, sadness) but fail to appreciate complex emotions that are based on 
attitudes and beliefs of others (surprise, embarrassment, pride) (Baron-Cohen et al., 1993; Prior \& Hoffman, 1990). Furthermore, Tager-Flusberg (2003) has demonstrated that ToM scores were significantly related to both scores on the socialisation domain of the Vineland Adaptive Behavior Scales and to social- and communication-symptom severity (as measured by the Autism Diagnostic Observation Schedule).

Researchers in favour of the ToM hypothesis of autism assert that the communication deficits and repetitive behaviours can be explained by ToM impairment. Tager-Flusberg $(1981 ; 1985 ; 1992)$ provides evidence that, phonological, semantic and syntactic development do not seem to be impaired but that there are impairments in the acquisition and use of mental state terms within autism. Furthermore, interpretation of the pragmatic aspects of language seems to be lacking in autism. The language of an individual with autism is often lengthy, inappropriate, and functional - all without regard for the nature of the listener. There is a striking absence of language use as a social tool purely to connect with others. Non-verbal communication deficits also fit the pattern of an absence of ToM. Instrumental gestures such as pointing to something wanted, a sign to tell others to be quiet or leave the room are seen in autism but pointing in order to direct the attention of others is absent (Attwood et al., 1988).

With respect to repetitive behaviours, the ToM hypothesis states that these are a coping strategy used in an attempt to reduce the anxiety that stems from the inability to understand the social world. 


\section{Problems with ToM Hypothesis}

While the ToM hypothesis provided a unified cognitive explanation for the deficits seen within ASD, further studies have revealed a number of issues with this theory. Firstly, the existence of a minority of individuals with ASD who are able to pass certain false belief tasks has undermined the universality of the deficit and hence has cast doubt on the hypothesis that a problem with ToM is the core deficit that underlies the condition. Happé noted, however, that this need not totally undermine the ToM hypothesis as there have been no cases of children with ASD passing first order false belief tasks at the appropriate mental age: on average a verbal mental age of nine years is needed (Happe, 1995). Such a deficit may also not be specific to ASD. Research has demonstrated that children older than four years with different disorders (e.g. non-signing deaf individuals) fail false belief tasks suggesting that a ToM deficit may not be a defining characteristic of ASD (Peterson, Wellman \& Liu, 2005).

Secondly, there seem to be at least two major problems with the false belief tasks. Firstly, it may be the case that passing false belief tasks may not solely be based on ToM, but might also require cognitive skills such as the ability to remember the sequence of the story, inhibit knowledge regarding the actual location of the object and correctly interpret the verbal task instructions and questioning (Birch \& Bloom, 2003; Bloom \& German, 2000). Both young typically developing children and children with ASD may find these task demands challenging due to deficits in executive functioning and/or reduced linguistic abilities (American Psychiatric Association, 1994; Astington \& Baird, 2005; Joseph \& Tager-Flusberg, 2004; Ozonoff et al., 1991; Ozonoff et al., 2004; Tager-Flusberg, 1981). 
In recent years, a non-verbal version of the false-belief task has been developed for use with infants (Onishi \& Baillargeon, 2005). They showed that 15month old typically developing infants were surprised when they saw an actor, who had not seen a toy being moved, searched in the correct location. This suggests that these infants had attributed a false belief to the actor and clearly contradicts the previous view that children below the age of 3 years cannot represent others' mental states (Perner, 1995; Saxe et al., 2004). Following these findings, Southgate, Senju and Csibra (2007) designed a similar non-verbal task that used eye-tracking equipment to measure anticipatory looking, and hence determine whether young children possess ToM, and confirmed that two-year-old children can correctly interpret an actor's false belief and subsequent behaviour. They then used this task with a group of children with ASD and showed that the clinical group were impaired on even this non-verbal task of false-belief attribution.

While this may have gone some way to remove confounding factors inherent due to task demands, the second problem concerns the observation that it is possible to correctly answer the questions on the false belief task without appreciating the mental states involved. Ability on false belief tasks does not seem to correlate with the level of social interaction and communication in children with ASD (Ozonoff \& Miller, 1995) and individuals with ASD who do pass the false belief tasks can still exhibit problems in related tasks such as attribution of mental states to geometric shapes (Abell et al., 2000) or to people (based on photographs of the eyes) (BaronCohen et al., 1997; Baron-Cohen et al., 2001).

While false belief tests should not be ruled out completely it is clear that there are still a number of issues to be resolved if the ToM hypothesis is to be accepted as a likely explanation for autism. 


\section{Executive Dysfunction}

The second major theory to be discussed here concerns executive function; the term used to describe goal-directed and future oriented processes that are thought to be controlled by the frontal lobes (Duncan, 1986). These behaviours include attention, flexibility, planning, generation, inhibition of pre-potent responses and working memory. All these involve the need to disengage from the external environment and employ mental models and representations to guide subsequent actions (Dennis, 1991). The ED hypothesis suggests that due to neuroanatomical dysfunction, difficulties with higher-order cognitive functions will be seen in individuals with ASD (Pennington \& Ozonoff, 1996; Verte et al., 2005) and that this deficit accounts for the behavioural features seen in autism (Russell, 1997; Turner, 1999a; Turner, 1999b).

The impetus for this line of research came from a study by Damasio and Maurer (1978) who demonstrated the similarities between the behavioural profile of autism and the symptoms of patients with neurological damage to frontal lobe structures. Both populations appeared inflexible, perseverative, overly focussed on details and unable to inhibit overlearned responses. The repetitive behaviours and rigidity - one of the diagnostic triad for autism - may therefore be caused by executive dysfunction. As discussed above, there is evidence for neuroanatomical abnormalities in the frontal lobes on individuals with ASD (Carper \& Courchesne, $2000 ; 2005)$ and reduced functional connectivity between frontal and parietal regions has also been highlighted in ASD during a task of executive functioning (Just et al., 2007). 
Executive function in autism has most commonly been tested using the Wisconsin Card Sorting Task (WCST) (Grant \& Berg, 1948) which relies on cognitive shifting ability. In this task, participants sort cards and are given feedback based on a rule that they have not been told. The feedback allows the rule to be ascertained but, without the participant's knowledge, the rule is changed every time ten cards have been sorted. Participants are scored based on how many perseverative responses they make. That is, the number of cards they sort based on a previously correct category despite being given negative feedback when the rule changed. The majority of studies have found that individuals with autism perform worse on the WCST, displaying increased perseveration and difficulties in shifting between sorting strategies (Bennetto et al., 1996; Ozonoff \& McEvoy, 1994; Prior \& Hoffman, 1990). Similar results are found when using Tower of Hanoi (Bennetto et al., 1996; Ozonoff \& McEvoy, 1994) or Tower of London tasks (Shallice, 1982) that involve planning and working memory. Individuals with autism show poor task performance relative to control individuals.

Unlike the ToM hypothesis which focussed on the behavioural profile of autism, ED attempts to locate the neurobiological substrate. It can, however, be linked to the symptom profile seen in the condition. The lack of flexibility resulting from ED could underlie the inability to engage appropriately in reciprocal social interaction and the problem with inhibiting prepotent responses could account for inappropriate behaviour. Russell and colleagues (1997) suggest that ED could be responsible for the mentalising and ToM deficits - an inability to disengage from the immediate context and switch over to internally driven behaviour (Hughes \& Russell, 1993). There also appear to be significant correlations between socialcognitive and executive function abilities (Grattan \& Eslinger, 1989; 1992). 
Specifically, Ozonoff found that executive function scores were highly correlated with mentalising ability in individuals with autism (Ozonoff et al., 1991) and executive function has been shown to be an accurate predictor of long term prognosis in autism (Berger et al., 1993). More recently, Hill and Bird (2006) showed that impaired performance on a set of executive function tasks (requiring planning, abstract problem solving and multitasking) was correlated with autistic symptomatology.

The suggestion from neuroimaging research that mentalising capability may be controlled by the prefrontal cortex (Baron-Cohen et al., 1994; Fletcher et al., 1995) could also link the social deficits to ED due to the proximity of the neural structures involved. Regarding the communication deficits in autism, the nature of speech seen in individuals with the condition is consistent with ED. The repetitive language and overall poverty of speech can be attributed to deficits in generating sequences of behaviour and a failure of inhibition that leads to the stereotypical perseverations. Adding weight to this argument is the demonstration that patients with frontal lobe damage appear to have similar communication impairments to individuals with autism. Problems are seen with metaphors, social inferencing and talking about the intentions of others (Frith \& Snowling, 1983; Happe, 1991). The problems individuals with autism have concerning the pragmatics of language are not, however, replicated in patients with frontal lobe damage (Bishop, 1993) and it is unclear how ED could account for such difficulties.

Repetitive behaviours, the final component of the diagnostic triad, can easily be accounted for by ED. A failure to generate or activate appropriate actions and an inability to inhibit the repetition of previously employed action plans is consistent with the rigid and repetitive nature of autism (Turner, 1997). 


\section{Problems with the ED Hypothesis}

The main issue that undermines the validity of the ED hypothesis of autism is the presence of executive function deficits in a number of other conditions and disorders. ED is seen in Parkinson's disease (Owen et al., 1995) obsessive compulsive disorder (OCD) (Head et al., 1989), schizophrenia (Axelrod et al., 1994), attention deficit hyperactivity disorder (ADHD) (Chelune et al., 1986), Tourette Syndrome (Gladstone et al., 1993) and Fragile X Syndrome (Mazzocco et al., 1997). The presence of ED in so many different conditions undermines the idea of a specific and causal relationship between ED and autism. It may be the case that ED is purely the result of a damaged brain - irrespective of the nature of that damage. On the other hand, Pennington and colleagues have argued that there are a number of ways in which different conditions can share the same underlying cognitive deficits (Pennington \& Ozonoff, 1996). They suggest that the different behavioural profiles of the conditions mentioned above reflect differing patterns of ED onset. Alternatively, it may be the case that the term 'executive dysfunction' is too broad and should be split into smaller, more specific, components. It is a possibility that different disorders are underpinned by deficits within difference executive function components.

Using this approach, Hughes, Russell and Robbins (Hughes et al., 1994) looked specifically at set-shifting ability in autism and revealed that there was a flexibility deficit. Inhibition was examined by Ozonoff and Strayer (Ozonoff \& Strayer, 1997) and results indicated that both motoric and cognitive inhibition was unaffected in autism. This particular pattern of executive functioning does seem to be distinct from other conditions that also show ED. For example, unlike autism, there 
appears to be a deficit in inhibition within ADHD (Aman et al., 1998), OCD (Enright \& Beech, 1993) and schizophrenia (Beech et al., 1989). Using this multi-component approach may therefore begin to highlight a specific deficit in executive function within autism that could be responsible for the condition.

\section{Weak Central Coherence}

The third major theory of autism is that weak central coherence (WCC) underlies the condition (Frith, 1989). WCC refers to a difficulty integrating component parts of a visual scene to create a coherent whole. Frith suggested that within autism there is a deficit in the cognitive system that is responsible for this process of extracting overall meaning, causing individuals with autism to process constituent parts of an item as separate entities rather than seeing the item in its entirety. The evidence that led to this theory comes not from observed deficits but from the superior performance that individuals with autism demonstrate on various tasks. One of the distinctive characteristics of autism is the uneven cognitive profile that is seen when administering IQ measures. While typical individuals show equivalent performance across all subtests, individuals with autism consistently show below average performance in the verbal tasks and above average performance in non-verbal measures such as block design and pattern construction (Shah \& Frith, 1983; 1993). An even more striking advantage is seen on The Embedded Figures Task (EFT) (Witkin et al., 1971). This involves the detection of a shape that is hidden within a picture, e.g. a triangle that is hidden in the line-drawing of a baby's pram. Both children and adults with autism seem to detect the target faster than their typically developing counterparts (Jolliffe \& Baron-Cohen, 1997; Shah \& Frith, 1983). 
It has been proposed that this theory of WCC can also explain the social and language deficits within the condition. Frith (1989) argued that in order to determine the mental states of others one needs to integrate information from many different sources: emotional expression in the eyes, tone of the voice etc. An inability to do this would undermine the development of theory of mind and subsequently affect social interaction. The same logic has been used to argue that language deficits in ASD could be due to WCC. In order to understand the meaning of speech, the context of a sentence must be taken into account. A difficulty in integrating the many aspects that allow context to be evaluated would lead to the literal interpretation and pragmatic difficulties that are often seen in ASD (Happe, 1991).

In more recent years, Happé has re-examined the idea of WCC as a deficit. She has suggested that central coherence is a cognitive style that varies (weak to strong) across the typical population; where individuals with autism show a cognitive style that is shifted towards the weak end of this continuous spectrum (Happe, 1999). This weak coherence style could manifest itself behaviourally as attention to detail, obsessive interests and resistance to change.

Problems with WCC

A number of studies have produced results that contradict the theory of WCC in ASD. Ozonoff, Strayer, McMahon and Filoux (Ozonoff et al., 1994) used a Navon task (hierarchical stimuli where small letters are arranged to form a large letter) to investigate WCC (see figure 1.1). They instructed participants to identify either the local or the global target and found that both typically developing children and children with autism showed a global advantage and a global interference effect. 
Figure 1.1 Example of the stimuli used within the Navon Task

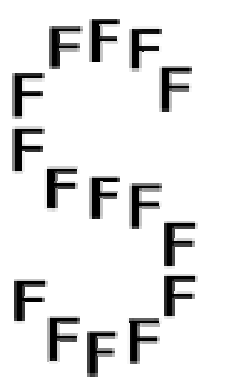

In an attempt to explore these findings, Plaisted, Swettenham and Rees (1999) used two versions of the Navon task. One version, similar to that employed by Ozonoff et al, was a selective attention task, involved detecting a target which could appear at the local (small letter) or global (big letter) level. The other, a divided attention task required participants to focus on the local or global level depending on the task instructions. In the divided attention task, results were in line with WCC; the children with ASD demonstrated a local advantage whereas typically developing children did not. Conversely, in the task of selective attention, both groups of children showed the typical global advantage and global interference effect. These results are consistent with those found by Ozonoff et al. (1994) and may undermine the validity of WCC as a theory that underlies ASD. Mottron and colleagues (Mottron et al., 1999; Mottron et al., 2003) also find evidence that global and configural processing is not altered in ASD, and suggest that the idea of local bias and global impairment within WCC may need to be re-examined. This is echoed by López and Leekam (2003) who conclude that children with ASD do not show the general difficulty in connecting context information and item information that would be predicted by the WCC theory. Mottron and Burack (2001) have proposed a model of perceptual functioning in ASD that offers an alternative to the WCC framework. The Enhanced Perceptual 
Functioning (EPF) model suggests that the superior performance seen by individuals with ASD on tasks outlined above is due to enhanced perception. Within the term 'perception' the authors include processes ranging from feature detection to pattern recognition and suggest that there is a superiority of perceptual flow of information in ASD. This leads to both positive symptoms, such as enhanced memory of the surface properties of visual patterns, and also detrimental effects such as a difficulty in controlling perceptual processes - which then disrupt the development of other abilities (Mottron et al., 2006). This idea of superior ability on low-level perceptual operations contrasts with the deficit in global processing that was proposed within the WCC model.

\section{Systemizing, Empathizing and the Extreme Male Brain}

Baron-Cohen puts forward a slightly different theory to account for this apparent difference in cognitive style between individuals with autism and the typically developing population. He defines two concepts, systemizing and empathizing, which form the two opposing extremes of a continuum of processing styles (Baron-Cohen, 2002). Both are mechanisms that are used to respond to change in the environment. Empathizing is the process used to respond to agentive change: where an object change is believed to be self-propelled and therefore the object is seen by the brain as an agent with a goal. This involves the attribution of mental states (mentalizing) in order to make sense of the change. Systemizing is the process used to respond to non-agentive change: a structured change (i.e. non-random) that is not self propelled. For example, a light switch causing the light to go off. When systemizing, we use the many observations of real-world occurrences to derive laws that can predict an event (Baron-Cohen, 2006). This works best for more predictable 
(lawful) changes with narrow variance and is therefore less useful when dealing with complex social behaviours. Baron-Cohen asserts that an individual's processing style is dependent on the balance between these two mechanisms; with men generally placed further towards the systemizing end of the spectrum and women towards the empathizing end. Within the general population he highlights the presence of 8 ascending levels of the systemizing mechanism (SM). Females mostly exhibit level 2 and males mostly exhibit level 3; both demonstrating a fairly even balance between systemizing and empathizing. Baron-Cohen suggests that above this level, hypersystemizing leads to autistic traits such as superior attention to details and resistance to change. Individuals with a SM at level four or five include those who excel at mathematics and science and those with Asperger's Syndrome. Levels six, seven and eight are seen in people with high functioning, medium functioning and low functioning autism respectively. He suggests that hyper-systemizing in autism leads to an inability to deal with systems that are less than $100 \%$ lawful - something that would cause the distinctive cognitive profile of ASD: reduced IQ and language delay but talent in systemizable areas.

\section{Summary}

It is clear from the discussion above that ASD is multi-faceted and complex with many potential aspects that may underlie the condition. Researchers are now beginning to move away from a grand unified theory of autism in favour of more specific hypotheses that address certain aspects of the autistic phenotype. Francesca Happé and colleagues have noted that despite decades of research, there is still very little unity between the core areas of impairment. In fact, the early epidemiological study which established the triad in the first place is the only full examination of the 
issue (Wing \& Gould, 1979). Happé et al. question the assumption that the three impairments must be explained together (Happe et al., 2006). Citing the evidence that, within the general population, the three autistic traits do not cluster together or show more than a modest correlation (Ronald et al., 2006a; Ronald et al., 2006b), they assert that the social deficits, communicative difficulties and rigid behaviours can be fractionated and should be studied separately. Furthermore, they note that studies have demonstrated different developmental trajectories for each of the triad components: with social and communicative deficits emerging earlier than repetitive behaviours (Charman \& Swettenham, 2001).

It is evident from the literature outlined here, that attempts to apply one hypothesis to all three core deficits have been largely unsuccessful. The cognitive theories that currently exist tend to focus on one component of the triad as a primary deficit and then extend the rationale to include the other aspects of the symptom profile. (E.g. ToM theory views social behaviour as the primary deficit whereas ED focuses on rigidity and repetitive behaviours). As yet, there is no unified cognitive account that posits a primary deficit which can account for the complete triad.

At a genetic level, this fractionation also seems to be the case. Using a sample of 3,000 twin pairs, Happé and colleagues demonstrated that each aspect of the triad appears to be highly heritable (Ronald et al., 2006a; Ronald et al., 2006b) and that genes that seem to contribute to variation in social ability are independent from those that contribute to variation in communicative skills (Ronald et al., 2005).

With all this in mind, there may be a case for abandoning both the search for a unified cognitive theory of autism and the search for 'autism genes' that underlie the whole condition (Happe et al., 2006). Instead, the future of autism research may lie in the examination of aspects of ASD which, while it may mean giving up on the 
idea of one overall 'cure', could also remove much of the heterogeneity within ASD; something that is a constant, and often prohibitive, difficulty with research in this field. 


\section{Chapter 2: Selective Attention and Perceptual Load}

In order to interact meaningfully with the world around us, it is vital that we have the ability to focus on particular aspects of the environment while ignoring others. Defined as 'selective attention', this ability is crucial given that the brain has limited sensory and information-processing systems which are constantly bombarded with an excess of information (Broadbent, 1958). "Without selective interest, experience is an utter chaos. Interest alone gives accent and emphasis, light and shade, background and foreground - intelligible perspective, in a word" (James, 1890).

Anecdotally, this "chaos" is often observed within ASD where individuals with the condition are seen to fixate inappropriately on seemingly irrelevant information in the environment and are unable to make sense of the world around them (Bryson et al., 1990; Hayes, 1987). The original definition of autism by Kanner made reference to attentional deficits associated with the condition (Kanner, 1943) and while a great deal of research has looked into attentional abnormalities, there is relatively little work that has focused specifically on selective attention and ASD (see Allen \& Courchesne, 2001; Burack et al., 1997 for a review). Based on recent advances within the field of attention research, this thesis seeks to investigate selective attention in ASD. Relevant aspects of mainstream selective attention research will first be discussed before considering the issue of attentional abnormalities within ASD. 


\section{Selective Attention}

Much of the research on attention has focussed on the ability to prioritise the processing of one aspect of the environment over another. As James (1890) remarked, "Millions of items of the outward order are presented to my senses which never properly enter my experience. Why? Because they have no interest for me. My experience is what I agree to attend to." Initially, selective attention was studied within the auditory modality following observations that, despite a barrage of noise, people at a party could successfully focus on the conversation that they were involved in without being distracted by other guests. Cherry (1953) used dichotic listening tasks to replicate this effect experimentally. He used headphones to play different auditory streams to each ear and asked participants to concentrate on the input to one ear only and repeat it back to him. Cherry then investigated what aspects of the message in the unattended channel listeners were aware of, and what affected how successfully one input could be shadowed. He found that only physical properties (pitch, volume etc) of the unattended stream appeared to be processed. Listeners were unaware of semantic content, individual words or even when the language changed (from English to reversed speech). Furthermore, supplying the two streams from different locations (i.e. to each ear) or using physically different voices (male vs. female) was sufficient to elicit effective shadowing of one channel only. Broadbent (1958) took these results and those from a number of other studies and put forward the first detailed theory of attention. He concluded that selection occurs after only basic physical properties of stimuli have been processed (see figure 2.1). 
Figure 2.1 Filter theory of early selection (Broadbent, 1958)

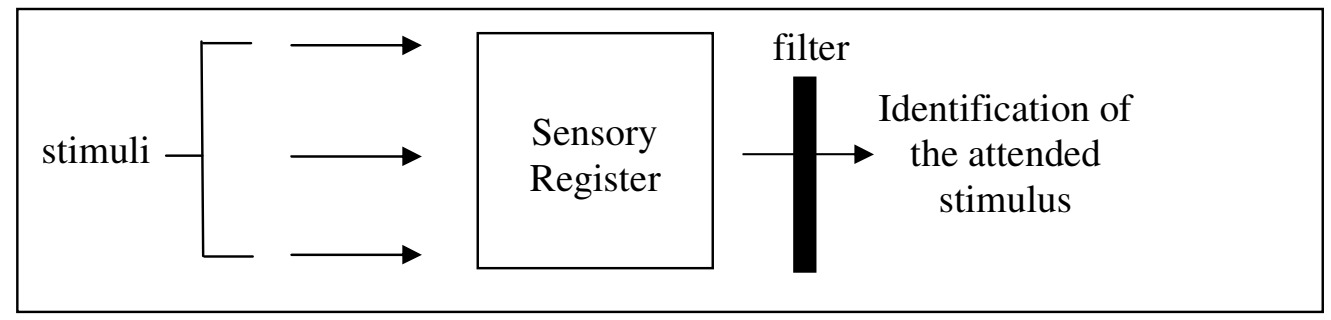

In his early selection model, Broadbent claimed that stimuli are first processed in a parallel fashion to extract physical attributes such as pitch, location and volume. The subsequent mechanisms for semantic identification, he argued, have a much smaller capacity and therefore incoming sensory information has to pass through a bottleneck before passing onwards for further processing. This bottleneck filters the stimuli in an all-or-nothing fashion (on the basis of physical attributes) to prevent more than one input from reaching the later processing stages at the same time. This idea fitted well with the conclusions drawn by Cherry (1953) regarding the very limited awareness of the unattended message.

There were, however, a number of observations that undermined Broadbent's model. Cherry described "The Cocktail Party Effect" where individuals who were seemingly focussed on a conversation would immediately detect their name being called on the other side of the room. Moray (1959) demonstrated this experimentally using dichotic listening tasks to highlight that listeners showed own name recognition in the unattended channel. Furthermore, in an experiment where participants were asked to "tap when you hear tap" in the attended channel, listeners were unable to tap when their name was presented simultaneously in the unattended channel (Corteen \& Wood, 1972). Thirdly, Corteen \& Dunn (1974) used fear conditioning to show that a galvanic skin response was elicited even when the 
relevant words were presented to the unattended side. Critically, words related to the fear-conditioned words elicited a reaction which implies some semantic processing of the unattended message.

In light of these factors, Deutsch and Deutsch (1963) proposed an alternative late-selection model based on the premise that all stimuli are fully analysed and then the most relevant stimuli are selected for awareness, memory and response. The limited capacity processing stages begin only after full semantic analysis has been performed (see figure 2.2).

Figure 2.2 Late-selection theory of attention (Deutsch \& Deutsch, 1963)

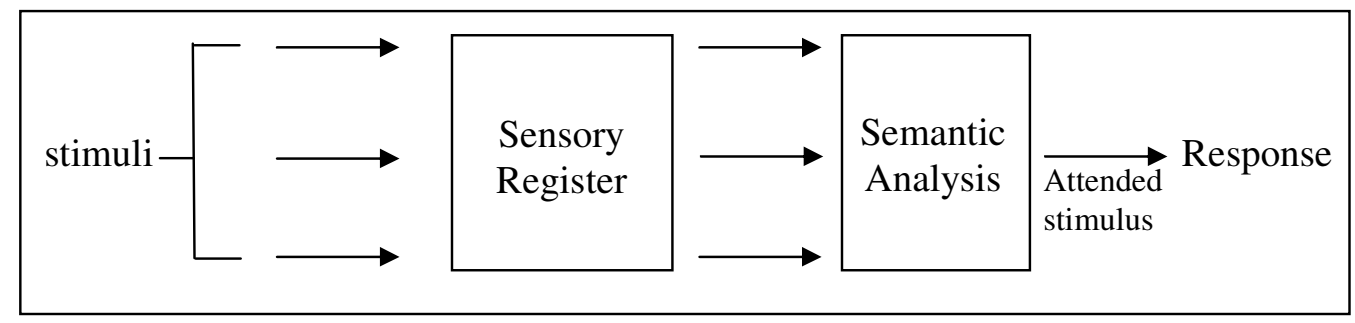

If this late-selection model is accurate then it follows that in dichotic listening experiments requiring participants to detect a target word in either ear, you would see no difference in detection rates between the attended and unattended stimulus. This, however, was not the case. It was shown that the shadowed message had a detection rate of $87 \%$ compared to $8 \%$ for the unattended message. (Treisman \& Geffen, 1967, as cited in Treisman, 1969)

Having undermined the late-selection model, Treisman put forward her Filter Attenuation Model (Treisman, 1964) which served as a compromise between the two conflicting selection theories. She asserted that rejected stimuli are not completely filtered out at the sensory register, but rather are attenuated. These reduced stimuli do 
not themselves reach the threshold for recognition but do activate detector units which are then accumulated. If the detector then identifies an item that is related to a recently activated unit (priming), then even partial activation may result in recognition of the "rejected" stimulus.

Figure 2.3 Filter Attenuation Theory of Attention (Treisman, 1964)

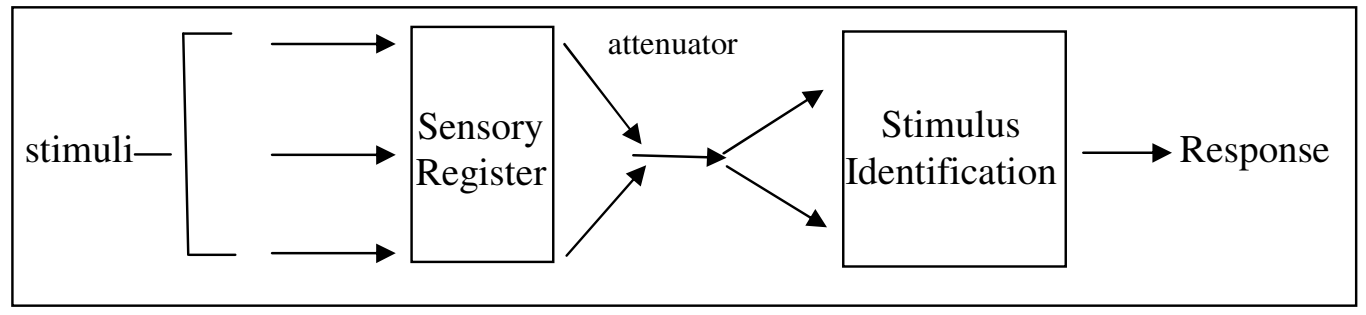

While the early theories outlined above were all grounded in hearing research, the focus has now moved towards selective attention within the visual domain. From the 1960s, researchers used visual filtering tasks to investigate visual attention. Sperling (1960) demonstrated that when participants are shown briefly a set of letters, they are unable to recall all of them. This was interpreted as evidence for limited capacity. Using similar methodology, Von Wright (1970) showed participants a set of characters and asked them to recall a subset based on various criteria. It was shown that performance was best when the subset was defined by colour, size, location or brightness rather than identification (letter vs. number or vowel vs. consonant).

Neisser (1976) and Rock and Gutman (1981) added further weight to the early selection argument by demonstrating that there was very little memory for the unattended visual stimuli. Rock and Gutman presented a pair of drawings (one red, one green) that were superimposed upon each other and asked subjects to give their 
opinions on the red figure. In a subsequent test, the number of green figures recognised was only just above chance level.

While such evidence seems compelling, there was also a body of work that suggested that while the unattended visual information could not be consciously remembered, this may not truly be evidence that it was not processed. Tipper (1985) used images based on Rock and Gutman's superimposed images and manipulated the relationship between the unattended image in one trial and the attended image in the next trial. He showed that the reaction times were slower when the ignored object was related to the subsequent attended object (negative priming). This provided evidence of late selection and indicated that there may well be full semantic processing of unattended visual stimuli. This is consistent with a number of studies based on the classic Stroop effect (Stroop, 1935) where, when naming the text colour of a word, an incompatible colour name (i.e. "RED" printed in green ink) elicits a slower response time than for a neutral word (i.e. "BED" printed in green ink). The difficulty of 'turning off' the semantic processing mechanism, even for an irrelevant word, is therefore observed.

In the 80s, Treisman and Gelade (1980) put forward a theory that differed from both the early and late selection models. Using visual search experiments they demonstrated that "feature search" (where the target and non target differ in a single salient property) was more efficient than "conjunction search" (where the target is defined by two criteria, e.g. colour and orientation). The former, it seemed was carried out in a parallel fashion while the latter appeared serial. (A more detailed account of visual search research is provided subsequently when discussing attentional abnormalities within ASD.) Based on these results, Treisman and Gelade (1980) created the "feature integration theory". The model asserts that simple 
individual features such as colour are represented in separate feature maps in a preattentional' stage and then attention is the process by which these maps are joined to facilitate identification of a given object.

Treisman's theory was very well received but was soon undermined by a number of studies. For example, research by Johnston \& McClelland (1974) and LaBerge (1983) seemed to imply that letters and indeed words behave as single units - something that goes against the Feature Integration Theory. The debate regarding the locus of selection and potential mechanisms continued.

An important new proposal was put forward by Lavie (1995) that may have helped to resolve the dispute. Lavie (1995) noted that during the 50s, 60s and early 70s, studies had found support for the early selection theories, whereas from the late 70s onwards selective attention studies seemed to find support for the late-selection approach. This, it was argued, may have been because different methodologies were used in the later experiments. Early studies, such as the dichotic listening experiments, used a 'filtering paradigm' which relied on bombarding subjects with a great deal of information to examine what stimuli are processed and which are ignored. Later studies, such as priming experiments, used the 'selective set paradigm' which involves presenting only a limited amount of visual information. Lavie suggested that the locus of selection is dependent on the perceptual load of the task in question - where perceptual load is defined as the amount of potentially task relevant information available in the external environment (Lavie, 1995). She used the Eriksen paradigm (accepted as a diagnostic tool to detect early or late selection) (Eriksen \& Eriksen, 1974) to observe the extent of distractor interference in tasks with varying levels of perceptual load. 
Participants sat in front of a computer and were presented with a central ring of letters on the screen. On every trial, a target letter - either an $\mathrm{X}$ or $\mathrm{N}$ - was present in the ring. Participants had to identify the target by pressing the corresponding key. Outside the ring of letters a slightly larger distractor letter was presented in one of two possible conditions: neutral or incompatible. A neutral distractor was a letter that had no association with the targets (i.e. $\mathrm{T}$ or L) whereas an incompatible distractor was the alternative target (i.e. $\mathrm{N}$ when the target in the ring was $\mathrm{X}$ ). It was expected that if the distractors were being processed then incompatible trials would elicit slower response times than neutral trials due to the interference. Perceptual load of the task was manipulated by altering the number of non-target elements in the central ring ('set size') (see figure 2.4). At each set size the extent of distractor processing and therefore the locus of selection - could be determined by comparing the reaction times for neutral and incompatible trials.

Figure 2.4 Examples of experimental stimuli used to investigate selective attention and perceptual load

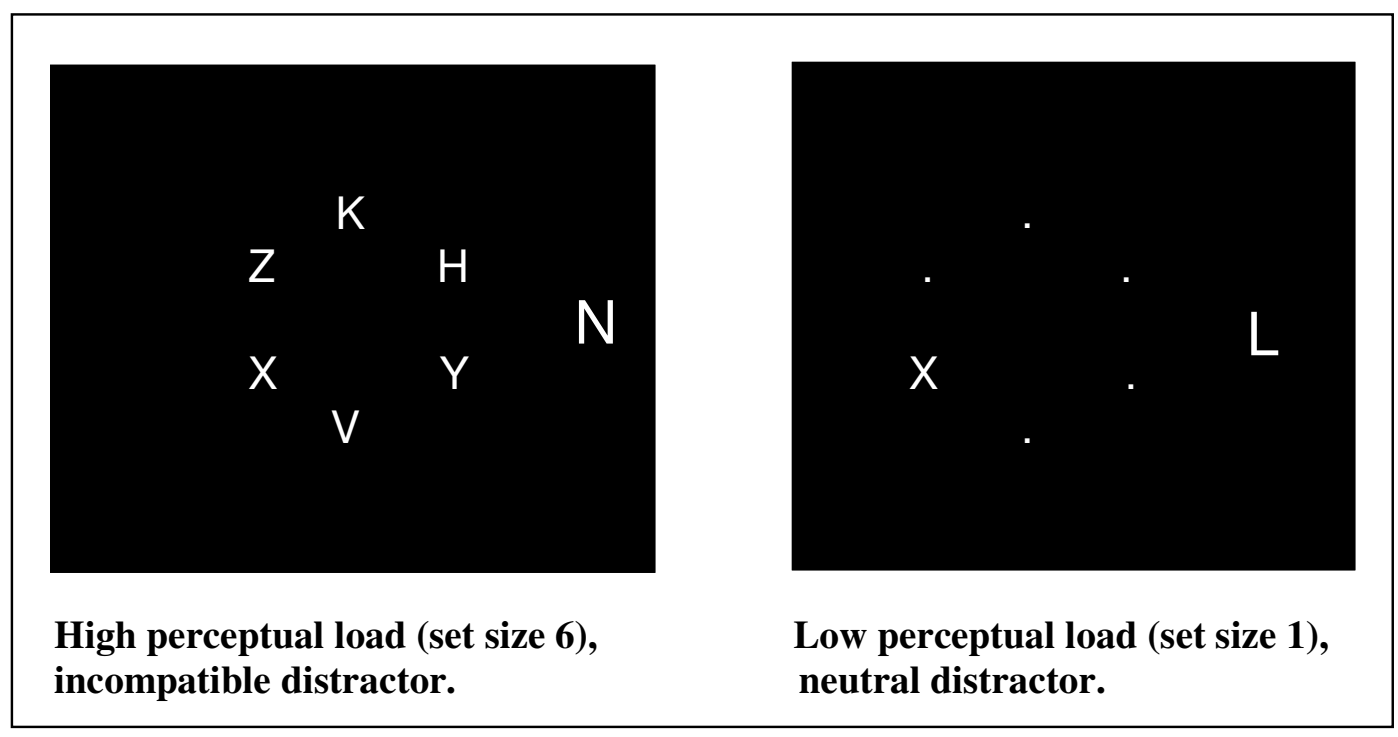


Lavie noted that when the perceptual load of a task is low, and does not exceed the perceptual capacity, then distractors are processed (late selection). Conversely, when the perceptual load of a task is high, interference from irrelevant distractors is eliminated, indicating that they are not processed (early selection) (Lavie, 1995). It should be noted at this point that perceptual load is not synonymous with taskdifficulty. Increasing the difficulty of a task by, for example, reducing the size or contrast of the targets may slow down performance but does not reduce distractor interference (Lavie \& de Fockert, 2003). The perceptual load theory of selective attention is based on the assertion that it is not possible to assign any less than the total capacity that is available at a given time (Lavie \& Tsal, 1994) so distractors are automatically processed when the central task does not exhaust all processing capacity.

A number of subsequent studies carried out both by Lavie et al. and other groups have corroborated these results. Neuroimaging studies have shown that the perceptual load of a task modulates the neural activity associated with irrelevant distractors. In one study, motion distractors were used and it was noted that responses in motion-selective cortices (MT, V1/V2) were only evoked in low-load conditions (Rees et al., 1997). In a further study by Rees et al. (Rees et al., 1999) it was seen that distractor words did not elicit neural responses in the high-load condition where participants were focusing on the central picture task. The perceptual load theory has also been investigated within subsets of the population. It has been demonstrated that for individuals aged between 65-79 years, the effect of distractors is eliminated at lower set sizes than for young adults (Maylor \& Lavie, 1998). Huang Pollock, Carr and Nigg (2002) showed that this is also seen in children - where early selection is seen at lower set sizes. They conclude that this is evidence 
of a reduced attentional capacity in these two population groups. If this perceptual load paradigm can indeed be used to investigate selective attention and perceptual capacity, then it may have a valuable application within research into atypical populations such as Autism Spectrum Disorder (ASD) where altered attentional abilities have often been highlighted (Burack et al., 1997).

\section{Autism and Attention}

Since the condition was first identified, there have been anecdotal reports of attentional abnormalities within ASD. Parents and clinicians have noted that individuals with ASD appear to fixate inappropriately on apparently irrelevant stimuli and perseverate on highly specific areas of personal interest. Gold and Gold (1974) stated that "the clinical syndrome of early infantile autism results from a malfunction in basic alerting and attentional mechanisms". The nature of a proposed attentional deficit has been based on various different attentional components and the research can be divided loosely on this basis. Within this chapter, literature concerning arousal, sustained attention, orienting and attention shifting in ASD will be outlined, before focussing on the more relevant issue of selectivity and filtering ability. It should be noted that a number of studies on attention and ASD have included a social component. The issue of social attention, however, will be dealt with separately in chapter six and therefore the discussion that follows here will only include experiments that used socially neutral stimuli. 


\section{Arousal}

A number of early studies looked at arousal in ASD in an attempt to explain the behavioural profile seen within the condition. Hutt and colleagues noted high levels of desynchronised electroencephalography (EEG) recordings in children with ASD and consequently stated that the autistic child is in a chronic state of overarousal (Hutt et al., 1964; Hutt et al., 1965), arguing that the repetitive behaviours and social withdrawal so often seen within the condition are attempts to reduce arousal. This rejection of sensory stimuli in order to cope with the overload of information that they face is a concept echoed by a number of other studies (Cohen \& Johnson, 1977; Kootz \& Cohen, 1981, van Engeland, 1984 as cited in Burack 1997). This theory was somewhat reinforced by Hermelin and O'Connor's (1970) demonstration that continuous monotonous stimuli cause arousal rather than habituation in children with autism, and Raymaekers et al's study (Raymaekers et al., 2004) that used a go/no go paradigm with varying rates of stimulus presentation to show that the ASD group had particular difficulty with the fast presentation rate condition - a finding they cite as evidence for over arousal in the condition. However, as is the case with most of the proposed attentional abnormalities in ASD, there is also contradictory research that suggests an alternative deficit in arousal mechanisms. Rimland (1964) claims that individuals with ASD in fact suffer from under-arousal, based on observation that children with ASD actively prefer conditions that involve greater stimulation (Graveling \& Brooke, 1978). Ornitz \& Ritvo (1968) modified these two conflicting arousal hypotheses to suggest that autism is "characterised by fluctuations between states of over and under-arousal resulting in a failure to modulate sensory intake adequately". The idea of an inability to effectively modulate arousal has also been supported by Kinsbourne (1987) who 
argues that within the same child there can be both extremes; producing both overand under-responsiveness.

In recent times the arousal theory has mostly been abandoned following the failure to obtain any significant supporting evidence (Burack et al., 1997; Goldstein et al., 2001). However, the idea of abnormal arousal levels may yet have relevance as a secondary outcome of an attentional deficit. Belmonte has presented electrophysiological data that suggest individuals with ASD compensate for impaired attention by applying a coarser mechanism of sensory gating. This results in a generalised level of arousal that heightens the response to all stimuli (Belmonte, 2000).

\section{Sustained Attention}

Closely related to the concept of arousal is that of sustained attention, defined as "the capacity to maintain attentional focus on a task over an extended period of time" (Bowler, 2007). The pattern of restrictive interests and obsessions seen in ASD suggests that there may well be enhanced capabilities for sustaining attention (Burack et al., 1997). The ability to sustain attention is most commonly measured using continuous performance tests (CPT) (see Rosvold, mirsky, sarason et al, 1956) which involve the rapid and repeated presentation of stimuli, within which the target stimuli are embedded.

Despite the theoretical basis for predicting otherwise, the studies on sustained attention in high-functioning individuals with ASD have failed to show any difference in performance levels when compared to control individuals (Buchsbaum et al., 1992; Casey et al., 1993; Goldstein et al., 2001; Pascualvaca et al., 1998; Siegel, Jr. et al., 1992). It has been suggested, however, that in some of these studies 
the way in which the groups were matched resulted in the ASD group having a considerably lower IQ than the control group (Burack et al., 1997). This may have masked any group differences that were present and led to an underestimation of sustained attention abilities in ASD. Furthermore, the low IQ individuals with ASD in Casey et al's study (1993), in line with the typical individuals, performed at a level close to ceiling.

Garretson, Fein and Waterhouse (1990) examined sustained attention in lower-functioning individuals with ASD and found that performance was at typical levels. The only difference between the groups was seen in a version of the paradigm that included a social reward component. The efficacy of social reinforcement was seen to decline at a faster rate for the individuals with ASD. This finding suggests that sustained attention may be linked to motivation - and therefore while no behavioural differences have been found in experimental situations, it may be the case that when faced with the subject of a particular interest, individuals with ASD may have enhanced capacity for sustained attention. Belmonte et al. (2003) has demonstrated that even when behavioural performance is equivalent, the brain regions activated in individuals with ASD during a task of sustained attention (ventral occipital and striate regions) are different from those of typical individuals (superior parietal, middle temporal, dorsolateral, prefrontal, premoter and medial frontal cortices).

\section{Orienting, Disengaging and Attention Shifting}

One of the most basic ways of assigning attention to a stimulus is to align sensory receptors to the relevant location. This process of orienting can be further divided into exogenous (reflexive), stimulus-driven, involuntary orienting and 
endogenous (controlled), information-based, voluntary orienting. Orienting behaviours can also either be overt: consisting of body, head and eye movements, or covert: involving the alignment of the mind's eye/ear. There are three main stages in the orienting process: disengaging, shifting and re-engaging (Posner \& Petersen, 1990). A number of studies have investigated these components in ASD. While there is no absolute consensus, the general interpretation of the literature is that automatic, exogenous orienting is intact (Burack \& Iarocci, 1995; Iarocci \& Burack, 2005; Minshew et al., 1999), while an impairment is seen in voluntary, endogenous orienting (see Bowler, 2007 for a review; Townsend et al., 1996a; WainwrightSharp \& Bryson, 1993). The deficit seems to manifest itself as a delay or a slowing of the orienting process (Harris et al., 1999; Townsend et al., 1996a; Townsend et al., 1996b; Wainwright-Sharp \& Bryson, 1993).

Attention disengaging and shifting, while part of the orienting process, has also been investigated specifically. Deficits in switching attention between locations (Casey et al., 1993; Wainwright \& Bryson, 1996; Wainwright-Sharp \& Bryson, 1993), object features and modalities (Courchesne et al., 1994; Rinehart, 2001) have been highlighted in ASD. Individuals were generally slower to shift attention between stimuli during the tasks. The presence of such an abnormality has been reinforced by the electrophysiological study by Belmonte (2000) who found altered Event Related Potential (ERP) responses to attention shifting in individuals with ASD. As mentioned above, his results suggest a more general level of arousal rather than the spatiotopically specific pattern of activation seen in typical adults. Other studies, however, have found no evidence of this deficit (Burack \& Iarocci, 1995; Iarocci \& Burack, 2004; Leekam et al., 2000). 
An alternative suggestion is that a problem disengaging attention underlies the abnormal orienting behaviour seen in ASD. Landry and Bryson (2004) used three computer screens to present stimuli both centrally and peripherally while a child fixated on the central screen. On certain trials the central stimulus remained on the screen while the peripheral stimulus was presented (disengage condition) while in others the central stimulus was removed before the presentation of the peripheral stimulus (shift condition). The authors found that children with ASD, verbal age matched typically developing children and children with Down syndrome all look longer to shift their gaze in the disengage condition, but this difference was greatest for the ASD group. This finding was taken as evidence for 'sticky attention': a difficulty in disengaging attention in ASD. This result conflicts with previous studies that do not find that individuals with ASD have a problem with disengagement (Townsend et al., 1996a; Townsend \& Courchesne, 1994) but the discrepancy may be due to differences in the age of children tested and nature of the stimuli used (Bowler, 2007).

\section{Selectivity and Filtering}

The most relevant set of studies with respect to the perceptual load theory of selective attention, and therefore this thesis, are those considering selectivity and filtering ability within ASD. Often cited is the theory that individuals with ASD show overselectivity. In one of the earliest studies regarding this issue, individuals with ASD were presented with complex stimuli composed of visual, auditory and tactile components (Lovaas et al., 1971). It was shown that, unlike typically developing children and children with intellectual retardation, children with ASD have a limited ability to use information from incoming stimuli and therefore attend 
only to one arbitrarily selected feature. This fits well with observations regarding the inability to integrate multiple components within the environment and the extremely focussed attention to detail (Lovaas et al., 1979). It has been suggested that this may be due to "tunnel vision" or an attentional gaze that is reduced in size - where individuals focus on an area that is overly contracted and therefore only allows processing of a reduced number of the relevant stimuli. This was demonstrated by (Rincover \& Ducharme, 1987) who separated the form and colour of a stimulus in space and found that, under these conditions, the individuals with autism responded to only one feature whereas control participants responded to both. The view that individuals with ASD have overly focussed attention has also been put forward by Courchesne and colleagues who interpret their findings from attention shifting paradigms as evidence of this (Townsend et al., 1996a; Townsend \& Courchesne, 1994). These findings may, however, have been due to developmental level and have not been replicated in older individuals (Burack et al., 1997; Enns \& Akhtar, 1989; Pasto \& Burack, 1995). Mann and Walker (2003) also disagree with Courchesne's interpretation, arguing instead that the deficits seen are more appropriately explained by a difficulty in broadening an existing focus of attention. Using a task that involved presenting participants with cross-hairs and asking them to indicate which line was longer (horizontal or vertical), they found that children with ASD showed a reduction in accuracy and an increase in response time when a large cross-hair followed a small one - but not the other way round.

As was discussed in the previous chapter, the issue of filtering and selectivity is commonly investigated by examining the impact of distractor stimuli on task performance. Burack (1994) showed that individuals with autism appear to have an inefficient attentional lens that fails to focus on the narrow visual field that is more 
appropriate for successfully attending to a stimulus. He asked participants to classify a target in the centre of the screen (either $\mathrm{O}$ or + ) in conditions that varied with respect to: a) presence/absence of a spatial window that enclosed the target and central region of the screen, b) number of non-target distractors and c) the distance between the target and the distractors. In the condition with no distractors, the children with autism showed more improvement with the presence of the window than typically developing children did. There was also an increased effect of distractors on the performance of the ASD group compared with the control group. These data lead to the hypothesis that children with ASD have an overly broad attentional field that does not contract appropriately. Burack's results could also be evidence of reduced filtering ability - something that is also suggested by Bryson, Wainwright-Sharpe and Smith (1990). Indeed, Ciesielski et al. (Ciesielski et al., 1990) used a divided-attention task (where participants had to identify the less frequently presented of two targets in visual and auditory modalities) and a filtering task (that involved ignoring the modality within which the target had been presented in the previous task) to show that participants with autism had more difficulty filtering out distracting stimuli than the corresponding control group. In this study the authors demonstrated that, while performing the tasks, there was an altered pattern of ERPs seen in the adults with ASD. It was seen that the selective attention-related potentials that indicate interchannel ( $\mathrm{Nd}$ and N270) and intrachannel ( $\mathrm{Nc}$ and P3b) stimulus selection were observed in the control group. Conversely, Nd, N270 and Nc were not seen in the ASD group and P3b was significantly reduced. Crucially, this difference was seen even in the few individuals with ASD for whom behavioural task performance appeared to be at normal levels (as measured by reaction time and accuracy data). The same group, however, published a subsequent study that 
demonstrated no behavioural differences between a group of high-functioning adults with ASD and a group of control adults with higher IQ than in the first study (Ciesielski et al., 1995) and later studies by Burack and colleagues (Burack et al., 1996; Iarocci \& Burack, 2004) also found no filtering deficits in ASD.

Neuroanatomical abnormalities in ASD have also been investigated in an attempt to clarify the seemingly contradictory evidence regarding selective attention ability within the condition. Studies using magnetic resonance imaging have revealed parietal abnormalities (most commonly, increased sulcal widths in superior posterior parietal lobes) in individuals with ASD (Courchesne et al., 1993; Heilman et al., 1993). It was hypothesised that these anatomical differences may explain the attentional profile seen in ASD (Townsend \& Courchesne, 1994). ERP studies were used to investigate this by measuring the P1 "attention effect" in response to visual stimuli in typical individuals and individuals with autism both with and without the parietal abnormalities: $\mathrm{P}+$ and $\mathrm{P}$ - respectively. The pattern of $\mathrm{P} 1$ responses indicated that $\mathrm{P}+$ individuals seem to show an enhanced attention effect at the attended location but narrow distribution of attention overall, whereas P- individuals show a broad area of reduced activation (Townsend \& Courchesne, 1994). In addition, the individuals with parietal abnormalities showed faster responses to the target stimuli than either the P-ASD individuals or the typical controls. This dichotomy may help resolve the conflicting reports of both over- and under-selectivity within ASD. The observation that a group of individuals with ASD show enhanced attention may also explain the superior performance that has been highlighted on selective attention paradigms such as visual search tasks. 


\section{Visual search}

Visual search tasks have become one of the most widely used tool in the study of selective attention (Huang \& Pashler, 2005). They involve presenting a target, hidden amongst non-target elements, and asking the participant to locate the target. There are two main types of visual search: feature- and conjunctive-search conditions. In the feature-search condition the target is defined by one distinct attribute (e.g. a red X among green Ns and blue Xs) and the time taken to detect the target is generally independent of the number of distractors present. In such cases, it seems that the search is carried out in a parallel fashion where information about all the items in the display is processed simultaneously (Treisman \& Gelade, 1980). In the conjunctive-search condition the target is defined by a combination of two features (e.g. searching for a red X among blue Xs and red Ts). In typical individuals, the response time to the target increases linearly with number of distractors. This is indicative of serial search - where one item is processed at a time until the target is located. Performance on visual search tasks has been used to examine processing capacity limits, based on the suggestion that the inefficiency of visual search seen as the number of stimuli increases is indicative of capacity limits (e.g. Nagy \& Sanchez, 1990).

Attentional abilities within ASD have been investigated by looking at visual search behaviour. Plaisted, O'Riordan and Baron-Cohen (1998b) carried out a series of studies where typically developing and ASD participants had to search for a target hidden amongst distractor items. In the feature-search condition there was no difference between the groups: the time taken for both the ASD and control individuals to find the target was independent of the number of distractors present, suggesting that the search was carried out in a parallel fashion. In the conjunctive- 
search condition the typical children showed the predicted rise in response times as the number of distractor stimuli increased. Conversely, the children with autism showed very little increase in response time and therefore seemed to be using parallel search mechanisms as with the feature-search tasks. In the following years, further studies were carried out to clarify these findings. Using more difficult tasks to eradicate ceiling effects that may have influenced the results from the 1998 study, it was seen that superior visual search behaviour is observed in both feature- and conjunctive-search tasks (O'Riordan et al., 2001). It is generally accepted that a search rate lower than $10 \mathrm{~ms}$ per item reflects parallel search, whereas higher rates suggest serial search (Davis \& Driver, 1998; Treisman \& Gelade, 1980). Serial search is also indicated by an approximate ratio of 2:1 target-absent to target-present search rate ratio - i.e. serial search will be exhaustive in target-absent trials but, in target-present trials, would end after searching, on average, $50 \%$ of the stimuli. In O'Riordan et al's study (2001) The pattern of response times for conjunctive search tasks of increasing difficulty was not consistent with parallel search (as hypothesised in the former study) but seemed to be more efficient serial search. It is also important to note that this ability was not simply the result of increased general functioning as two groups were matched for IQ. More recently, this superior visual search has also been demonstrated in adults with ASD (O'Riordan, 2004).

O'Riordan et al. (2001b; 2001) interpreted the results from these visual search studies in a number of ways. Firstly, superior visual search may be due to an increased ability within ASD to discriminate between similar items. An alternative suggestion was that children with ASD have increased 'inhibition of return' whereby they have better memory for distractor locations that have already been inspected and therefore are more proficient due to reduced likelihood of revisiting a non-target 
item. Joseph and colleagues, however, have shown that the superior search behaviour is unlikely to be due to memory ability. They compared search efficiency in dynamic and static conditions. If memory is a factor in search ability then the efficiency in the dynamic condition should be lower (due to the inability to memorise visited locations). While the ASD group responded faster overall, there were no apparent differences in the search efficiency or the eye movement patterns of children with ASD and typical controls (Horowitz et al., 2007; Joseph et al., 2009).

It seems that more evidence has come down on the side of the former suggestion regarding increased discrimination between similar items. A series of tasks where the similarity between the target and the distractors was manipulated demonstrated that discriminability was the rate determining factor in visual search. The increased target-distractor similarity did not adversely affect the ASD group to the same extent as the control group indicating that the superior performance by individuals with ASD on visual search tasks is likely to be due to an increased ability to discriminate between items (O'Riordan \& Plaisted, 2001b; O'Riordan \& Plaisted, 2001a)

Indeed this has been backed up by other studies looking at responses of individuals with ASD to highly similar novel stimuli (O'Riordan \& Plaisted, 2001b; Plaisted et al., 1998a) and by Kemner et al. (Kemner et al., 2008) who showed that the individuals with ASD made fewer fixations than typical controls (indicative of enhanced discriminability).

One intriguing possibility is that the superior visual search abilities in ASD could be explained in the context of Lavie's (1995) perceptual load theory of selective attention. Given that the tasks used by Lavie and colleagues to test the perceptual load theory involve visual search, the two should be intrinsically linked; 
although the relationship between them is a little counterintuitive. When applying the perceptual load theory to visual search, it would be predicted that more efficient search results in more distractor processing. When the search is 'easy' (i.e. when distractors and targets are dissimilar and therefore easily distinguishable) the perceptual load is low as fewer aspects of the non-target stimuli are relevant. Perceptual capacity is therefore left over and results in unintentional processing of irrelevant distractor items. This outcome was demonstrated experimentally by manipulating the difficulty of a search task (dissimilar target and non-targets vs. similar target and non-targets) and evaluating the level of distractor interference in each condition. In the 'easy-search' condition, distractor interference was seen whereas this was eliminated when the search was more difficult (Lavie \& Cox, 1997).

It is possible therefore, that the enhanced performance may be a result of increased perceptual capacity in ASD. If an individual is able to process a greater number of visual stimuli simultaneously, then they will be able to locate the target item more quickly and will demonstrate more efficient visual search patterns. It may be the case therefore, that individuals with ASD can process more items within the visual search display before their capacity becomes saturated.

The research presented here will explore this hypothesis by re-examining the issue of selective attention in ASD with respect to the perceptual load theory of selective attention and cognitive control (Lavie, 1995), and will investigate whether the varying pattern of attentional behaviour seen in ASD is more appropriately explained within this theoretical framework. 


\section{Chapter 3: Selective Attention and Perceptual Load in ASD}

\section{Introduction}

It is clear from the discussion in the previous chapter that the attentional abnormalities that are seen within ASD are diverse and often contradictory. Among the various reports of altered attention, it has been suggested that a reduced ability to filter out irrelevant information could be causing individuals with ASD to be bombarded with information - leading to over arousal (Dawson \& Lewy, 1989). Indeed, Ciesielski et al. (1990) showed that participants with autism had more difficulty filtering out distracting stimuli than the corresponding control group. Ciesielski et al. (1990) also showed that there was an altered pattern of event related potentials (ERPs) in the autism group during selective attention tasks in both visual and auditory modalities. Similarly, Burack (1994) noted that there was an increased effect of distractors on the performance of the ASD group compared with the control group but concluded that individuals with ASD appear to have an inefficient, overly broad attentional lens that fails to contract appropriately when attending to stimuli.

Given the theory put forward by Lavie et al. (1995), it is now important to reexamine the issue of selective attention in ASD within this new frame of reference. Based on the studies outlined above that demonstrate an increased effect of distractor stimuli on individuals with ASD, it was predicted that when compared to the control group, the ASD group would require a higher level of perceptual load to eliminate distractor processing. According to load theory such a finding would indicate increased perceptual capacity in individuals with ASD. 
In the first experiment of this thesis, a version of the Eriksen flanker paradigm (Eriksen \& Eriksen, 1974) similar to that employed by Maylor and Lavie (1998) was used to examine the extent of distractor interference at varying levels of perceptual load. A clinical and control group of participants took part in a two-way forced-choice reaction time task and were required to identify a target letter presented within a circle of letters in the central area of a computer screen. The perceptual load of the task was manipulated by adding non-target letters to the circle. There was also a distractor letter presented - offset from the central letter circle which participants were told to ignore. The distractor could either be incompatible or neutral (see method section for details). If the distractor letter is processed (late selection), then a slower response time will be seen for trials with incompatible distractors than for those with neutral distractors (which should not affect the response times). Conversely, if the distractors are being ignored (early selection), then the distractor type will have no differential effect on the response times.

It was predicted that, in line with Lavie (1995), the typical adults would show distractor interference at low levels of perceptual load (set size 1 and 2) whereas this interference would be eliminated as the perceptual load of the task was increased (set size four and six). For the ASD group it was predicted that while a pattern of interference modulated by perceptual load would still be seen, the distractor effects would persist at higher levels of perceptual load than for the control group.

\section{Participants and Diagnosis}

Participants in this study were 15 adults with ASD and 25 typical adults. The participants with ASD were recruited by advertising on the National Autistic Society (NAS) website, handing out information packs at Prospects (NAS employment and 
training service for people with autism and Asperger syndrome who wish to work) and contacting people in online communities and on social networking websites. Control participants were recruited from undergraduate and postgraduate courses at University College London and through social networking websites. A number of the participants took part in more than one of the studies presented within this thesis (see appendix I for details of participant overlap across studies). One individual with ASD and two control individuals were removed from the sample because their error rates were higher than 2.5 standard deviations above the relevant group mean. All participants in the ASD group had received a clinical diagnosis of autism or Asperger's Syndrome (AS) (3 autism, 11 AS), based on criteria listed in DSM-IV (American Psychiatric Association, 1994), from a trained, independent clinician. Diagnosis was then confirmed by assessment with module 4 of the Autism Diagnostic Observational Schedule (ADOS) (Lord et al., 2002). None of the participants suffered from any other mental or neurological disorder and all had normal, or corrected-to-normal, vision. Groups were matched for non-verbal IQ using a sub-scale from the Wechsler Abbreviated Scale for Intelligence (WASI) (Wechsler, 1999) (see table 3.1).

Table 3.1 Descriptive Statistics for each Group

\begin{tabular}{lccccc}
\hline Group & Statistic & $\begin{array}{c}\text { Age } \\
\text { (years: } \\
\text { months) }\end{array}$ & $\begin{array}{c}\text { WASI } \\
\text { vocabulary } \\
\text { subtest }\end{array}$ & $\begin{array}{c}\text { WASI } \\
\text { matrix } \\
\text { reasoning }\end{array}$ & $\begin{array}{c}\text { WASI } \\
\text { full scale IQ } \\
\text { (2 subtests) }\end{array}$ \\
\hline ASD $(\mathrm{n}=14)$ & mean & $23: 6$ & 64.1 & 52.9 & 115 \\
7 males & S.D & $4: 4$ & 7.0 & 8.4 & 12.3 \\
7 females & range & $18: 10-30: 8$ & $58-79$ & $39-67$ & $97-136$ \\
\hline Control $(\mathrm{n}=23)$ & mean & $26: 7$ & 65.7 & 56.9 & 119 \\
13 males & S.D. & $2: 2$ & 6.4 & 7.6 & 11.1 \\
10 females & range & $21: 5-30: 0$ & $52-74$ & $41-67$ & $101-138$ \\
\hline
\end{tabular}


Independent-sample t-tests indicated that there was no significant difference between the verbal, non-verbal and full scale IQ scores of the two groups (all $\mathrm{p}$ values $>0.14$ ).

\section{Ethical Considerations}

Ethical approval for all the studies within this thesis was obtained from the UCL ethics committee. All participants were given an information sheet about the tasks and had the study explained to them orally. Individuals were only included in the study once they had given written consent. Participants were informed that they could withdraw from the study at any time without penalty. This was the case for all the studies presented within this thesis.

\section{Methodology}

\section{Apparatus and Stimuli}

Computer based stimuli were created using visual basic and were run on a custom built small form desktop computer and displayed on a ProLite 15" flat LCD screen (1280 x 1024 pixel resolution, 2ms response rate). The participant's chair was positioned such that viewing distance was $60 \mathrm{~cm}$.

The task was a response competition paradigm based on Maylor \& Lavie (1998) where participants were required to respond to a relevant target while ignoring irrelevant distractors.

Stimuli were presented in light grey against a black background. Following the presentation of a fixation cross in the centre of the screen for $1000 \mathrm{~ms}$, a target letter $(\mathrm{X}$ or $\mathrm{N})$ was presented in any of six positions that were positioned around the circumference of an imaginary circle with radius of $2.1^{\circ}$ visual angles from the 
fixation point. The other positions in the ring were occupied by a non-target letter $(\mathrm{Z}$, $\mathrm{H}, \mathrm{K}, \mathrm{Y}$ or $\mathrm{V}$ ) or marked by a small dot depending on the condition. Target and nontarget elements measured $0.4^{\circ}$ x $0.6^{\circ}$ visual angles. A slightly larger distractor letter $\left(0.5^{\circ} \times 0.9^{\circ}\right)$ was presented on the right or left of the ring of letters, $4.3^{\circ}$ from the centre (see figure 3.1).

The perceptual load of the task was manipulated by changing the number of non-target letters in the ring to create trials with set size of one (only the target letter in the ring), two (target + one other non-target in the ring), four (target + three nontarget elements) and six (target + five non-target letters). The irrelevant distractors were either neutral (unrelated to the target letter: $\mathrm{T}$ or L) or incompatible (an $\mathrm{X}$ distractor when the target is $\mathrm{N}$ and vice versa). The experimental display was presented for only $100 \mathrm{~ms}$ in order to preclude voluntary eye movement.

Six blocks of 72 trials were created with each set size and distractor condition appearing equally. The target and non-target letters were equally likely to appear in each of the six ring positions and the proximity to the distractor letter was counterbalanced. For set sizes two and four, the position of the group of letters and the position of the target within the group (edge/middle) was also counterbalanced. 
Figure 3.1 Examples of Experimental Stimuli

3.1a: Example of a high perceptual load trial with an incompatible distractor. In this case the set size is six and the target is X. 3.1b: Example of a low perceptual load trial with a neutral distractor. In this case the set size is one and the target is X.

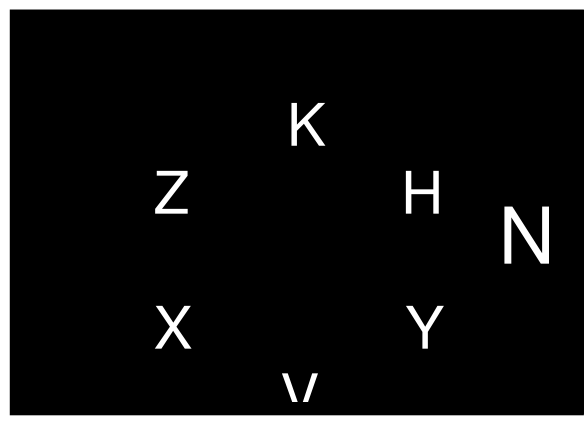

Figure 3.1a

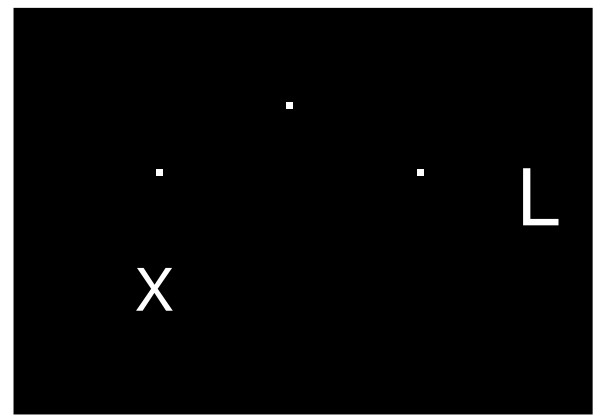

Figure $3.1 b$

\section{Procedure}

Participants were told that they would see a ring made up of letters in the centre of the screen and that one of those letters would be either an X or an N. They were told to press the $\mathrm{X}$ key if an $\mathrm{X}$ was present and the $\mathrm{N}$ if an $\mathrm{N}$ was present. It was emphasised that they should ignore the letters that were presented outside the ring. Following a set of practice trials, the participants completed the six experimental blocks and were able to take breaks between blocks if required. Every response was followed by a $1000 \mathrm{~ms}$ inter-trial interval and incorrect responses elicited a brief computer tone. The accuracy and response time for each trial was recorded by the computer program and subsequent comparison of reaction times for the two distractor trial types within high and low load conditions allowed the influence of distractors to be ascertained. 


\section{Results}

Reaction times above $3000 \mathrm{~ms}$ were discarded and median correct RT and error rates for each distractor condition at set size one, two, four and six were calculated. All incorrect trials were excluded from further analyses. An analysis of variance (ANOVA) was performed on the median correct RTs with group (ASD vs. control) as the between-subjects factor and distractor congruency (neutral vs. incompatible) and set size (one, two, four \& six) as within-subject factors (see table $3.2)$.

Table 3.2 Overall mean RTs (ms) and standard deviations (SD) for the two groups under each condition

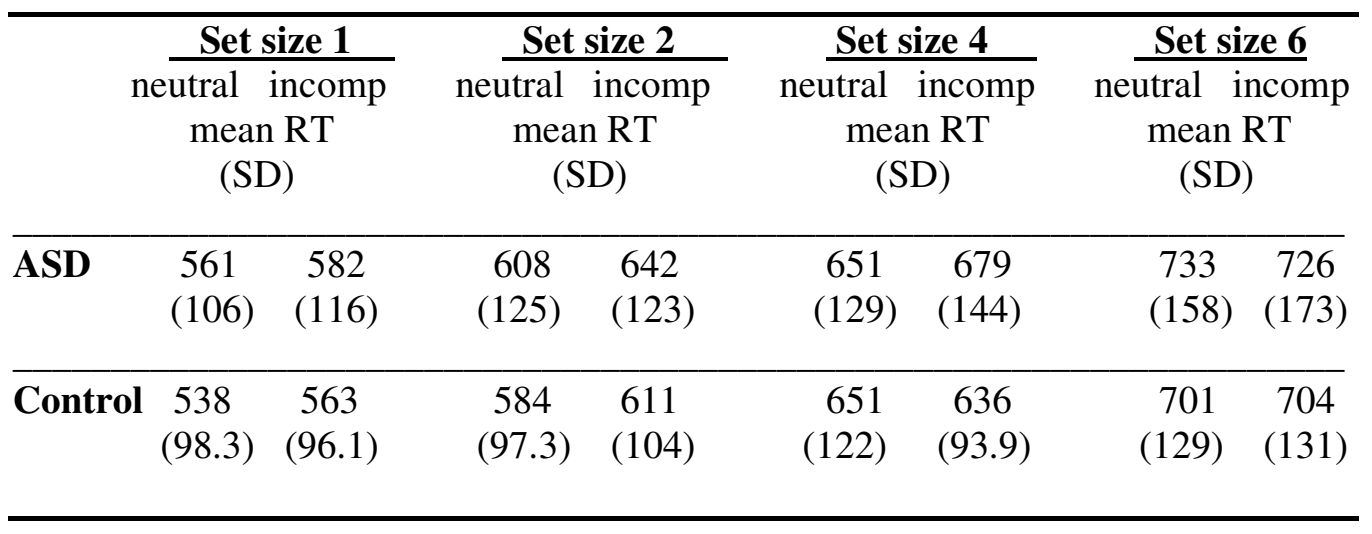

There was a significant main effect of set size indicating that participants were slower to respond to trials with higher set sizes $\left(F(3,105)=93.10, p<0.001, \eta_{\mathrm{p}}{ }^{2}\right.$ $=0.75)$. There was also a main effect of distractor congruency $(F(1,35)=18.09, \mathrm{p}<$ $\left.0.001, \eta_{\mathrm{p}}{ }^{2}=0.34\right)$ reflecting the fact that RTs were faster for neutral trials than for incompatible trials. These two main effects are reassuring as they imply that perceptual load had been effectively manipulated and that participants were engaging with the task in the way that was intended. 
There was no main effect of group $\left(F(1,26)=0.41, p=0.539, \eta_{p}^{2}=0.10\right)$, the reaction times were not significantly slower or faster in either group. This indicates that any differences between the groups are not due to a generalised reduction in processing speed within ASD.

Most importantly, there was a significant interaction between set size and distractor congruency $\left(\mathrm{F}(3,105)=4.23, \mathrm{p}=0.007, \eta_{\mathrm{p}}{ }^{2}=0.11\right)$ and a significant threeway interaction between group, set size and distractor congruency $(F(3,105)=2.79$, $\left.\mathrm{p}=0.044, \eta_{\mathrm{p}}{ }^{2}=0.07\right)$. The two-way interaction implies that the effect of distractor type varies with set size. The three-way interaction suggests that the two groups are showing a different pattern of congruency effects across the set sizes. These results can be seen more clearly in figure 3.2.

Figure 3.2 Difference between RTs for neutral and incompatible trials at each set size

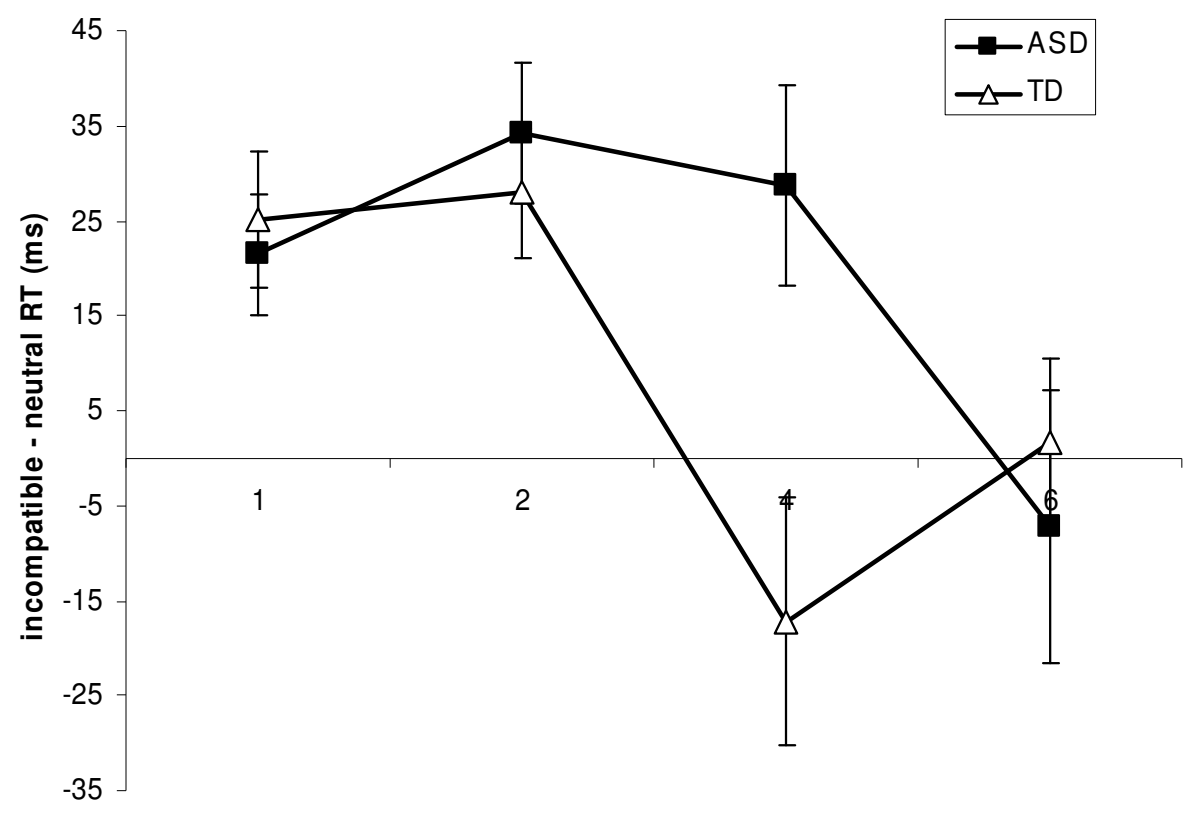

set size 
Using t-tests to explore the interactions, it can be seen that for the control group, there was a significant effect of distractor congruency at set sizes one $(\mathrm{t}(22)=-$ $3.470, \mathrm{p}=0.002)$ and two $(\mathrm{t}(22)=-3.925, \mathrm{p}=0.001)$ but no significant effect at set sizes four $(\mathrm{t}(22)=1.191, \mathrm{p}=0.246)$ and $\operatorname{six}(\mathrm{t}(22)=-0.308, \mathrm{p}=0.761)$. For the ASD group, there was a significant effect of distractor congruency at set sizes one $(t(22)=-$ $3.355, \mathrm{p}=0.005)$, two $(\mathrm{t}(22)=-4.619, \mathrm{p}<0.001)$ and four $(\mathrm{t}(22)=-2.751, \mathrm{p}=0.017)$ but no effect at set size six $(\mathrm{t}(22)=0.499, \mathrm{p}=0.626)$.

The difference between the two groups is therefore based on the lingering interference effect at set size four in the ASD group and the elimination of this effect at set size four in the control group. The latter appears to be due to the reduction in RTs for incompatible distractors within the control group, resulting in equivalent RT's for neutral and incompatible trials at that set size (see figure 3.3).

Figure 3.3 Mean RTs for neutral and incompatible trials at each set size for the control group (figure 3a) and ASD group (figure 3b)

\section{Figure 3.3a - Control Group}

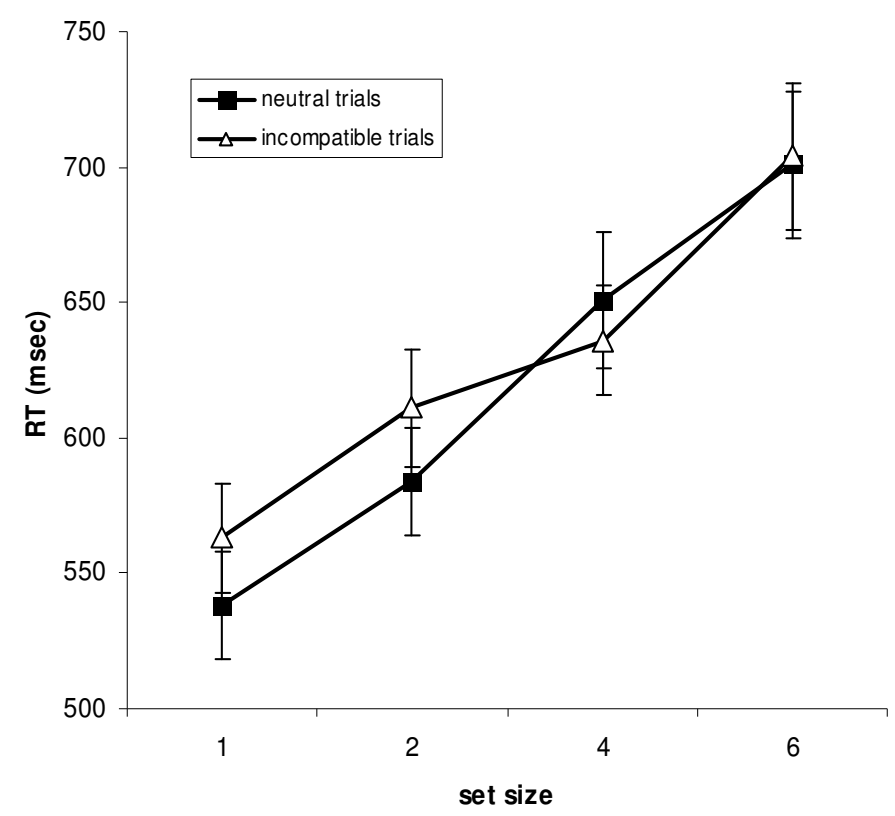


Figure 3.3b - ASD group

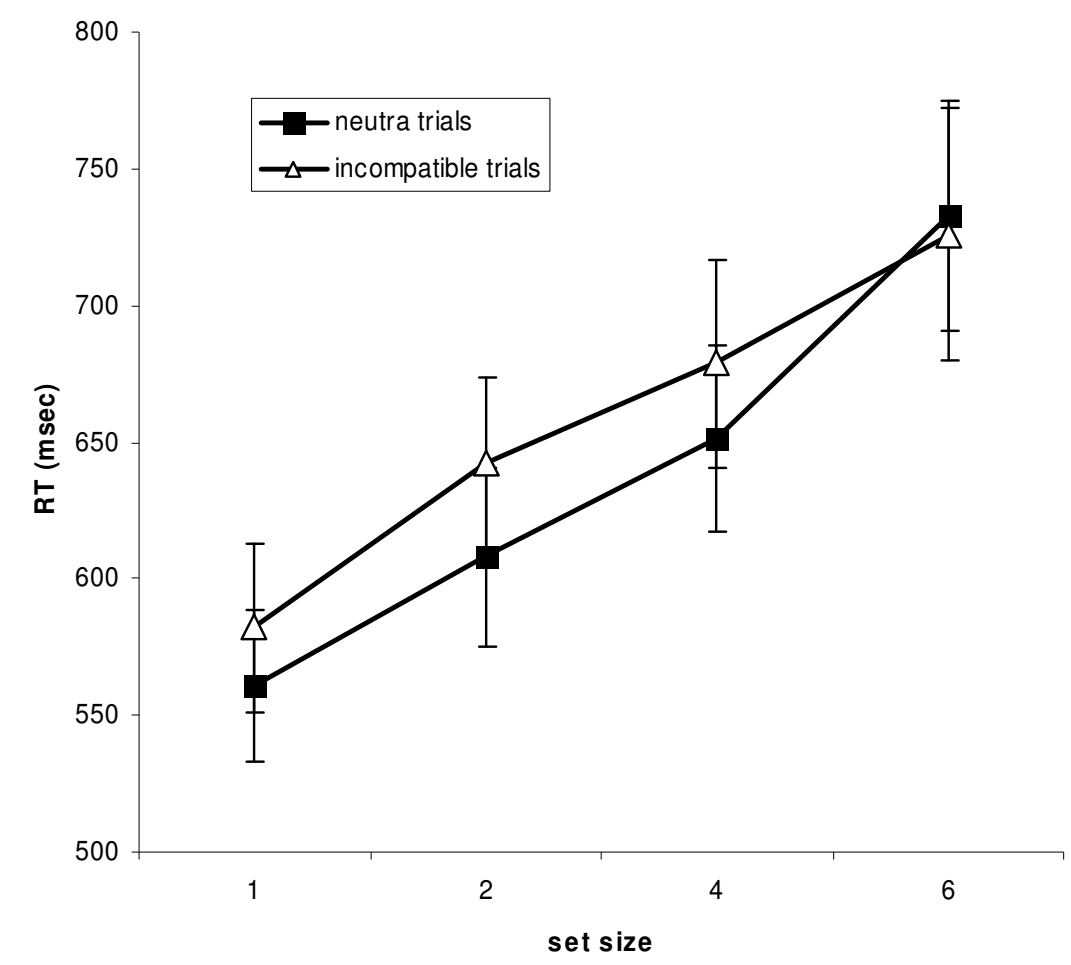

Search Slopes

The data from the neutral condition was examined in order to determine whether the visual search behaviour was different in the two groups (see figure 3.4). The difference in response times between set size one and the other set sizes was used to calculate an average search rate (ms per item). The average search rate for the ASD group was $36.5 \mathrm{~ms}$ per item, and for the control group was $34.8 \mathrm{~ms}$ per item. An independent samples t-test indicated that there was no significant difference between these two rates $(\mathrm{t}(4)=0.17, \mathrm{p}=0.873)$. 
Figure 3.4 Response times for neutral trials at each set size

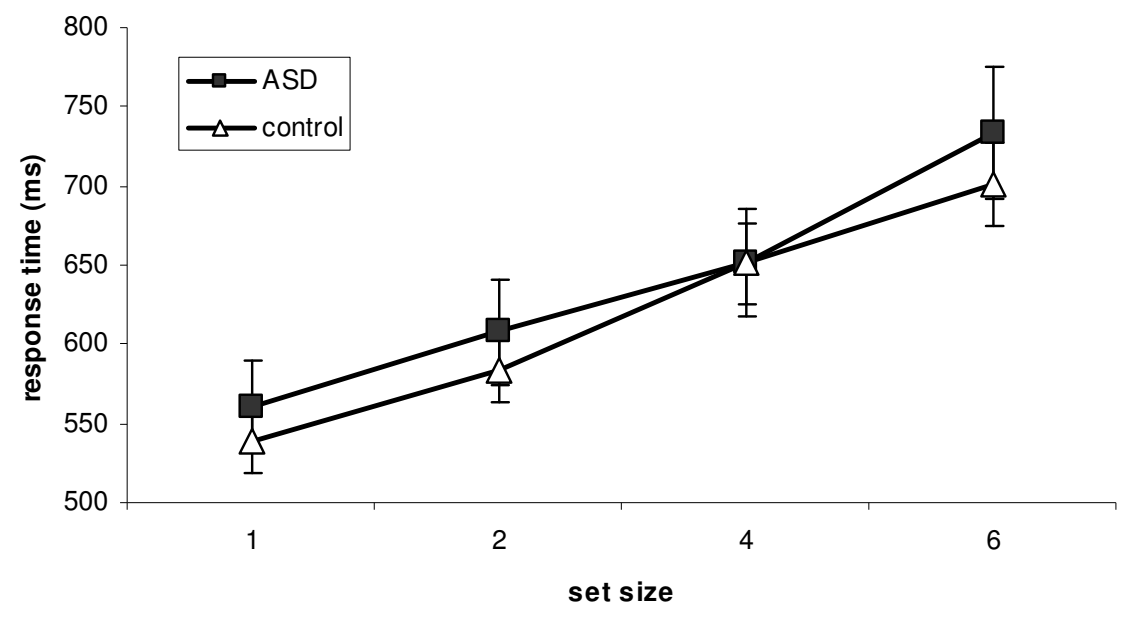

\section{Accuracy data}

Analysis of variance was performed on the number of errors for each group across each condition (see table 3.3 for absolute values).

Table 3.3 Accuracy rates and standard deviations for the two groups under each condition

\begin{tabular}{|c|c|c|c|c|}
\hline \multicolumn{2}{|r|}{ Set size 1} & Set size 2 & Set size 4 & Set size 6 \\
\hline & $\begin{array}{l}\text { neutral incomp. } \\
\text { Accuracy } \\
\text { (SD) }\end{array}$ & $\begin{array}{l}\text { neutral incomp. } \\
\text { Accuracy } \\
\text { (SD) }\end{array}$ & $\begin{array}{l}\text { neutral incomp. } \\
\text { Accuracy } \\
\text { (SD) }\end{array}$ & $\begin{array}{l}\text { neutral incomp. } \\
\text { Accuracy } \\
\text { (SD) }\end{array}$ \\
\hline ASD & $\begin{array}{cc}0.944 & 0.889 \\
(0.048) & (0.091)\end{array}$ & $\begin{array}{cl}0.852 & 0.907 \\
(0.087) & (0.104)\end{array}$ & $\begin{array}{ll}0.815 & 0.815 \\
(0.078) & (0.104)\end{array}$ & $\begin{array}{cc}0.665 & 0.694 \\
(0.057) & (0.103)\end{array}$ \\
\hline on & $\begin{array}{rl}\text { ol } 0.944 & 0.977 \\
(0.048) & (0.098)\end{array}$ & $\begin{array}{cl}0.944 & 0.870 \\
(0.059) & (0.120)\end{array}$ & $\begin{array}{ll}0.852 & 0.778 \\
(0.062) & (0.120)\end{array}$ & $\begin{array}{cc}0.741 & 0.704 \\
(0.091) & (0.127)\end{array}$ \\
\hline
\end{tabular}


There were main effects of set size $\left(F(3,105)=151.82, p<0.001, \eta_{\mathrm{p}}{ }^{2}=0.81\right)$ and distractor congruency $\left(\mathrm{F}(1,35)=8.29, \mathrm{p}=0.007, \eta_{\mathrm{p}}{ }^{2}=0.19\right)$ indicating that more errors are made at higher set sizes and with incompatible distractors. Overall, there was no significant difference between the number of errors made by each group $\left(\mathrm{F}(1,35)=0.48, \mathrm{p}=0.494, \eta_{\mathrm{p}}{ }^{2}=0.01\right)$. There were also no significant interactions between congruency, set size and group.

\section{Discussion}

As predicted, the individuals with ASD seem to be processing the distractors at higher levels of perceptual load than the control adults. There are a number of factors that can influence performance on a selective attention task and give rise to the difference seen in this study. Firstly, the ASD individuals may have a reduced filtering ability. Subjects who cannot effectively assign their attention to the central task and exclude irrelevant information would show this pattern of increased distractibility. Likewise, if the attentional lens was overly diffuse such that individuals with autism were unable to contract it sufficiently to block out the flanking distractor letters, then increased distractibility would be seen. A deficit of this nature which, regardless of perceptual load, prevents an individual from effectively assigning their attention to the central task would be reflected by an overall drop in accuracy and/or speed of performance on the task in question.

Given that there was no significant difference between the reaction times and error rates of the two groups the data from this study does not support either of these conclusions. The results are also not likely to be due to any difference in the pattern of eye movements between the two groups. While no gaze-tracking data was collected, the presentation of the experimental stimuli was brief (100ms) in order to 
preclude voluntary eye movement. The absence of any difference between the visual search rates of the two groups also suggests that the altered pattern of task performance in the ASD group is not due to the superior visual search abilities that have been highlighted within the condition (O'Riordan \& Plaisted, 2001a; O'Riordan et al., 2001; Plaisted et al., 1998c). It is interesting that there was no replication of the enhanced visual search behaviour that has been previously been reported. This may, however, be due to two factors. Firstly, at the low set sizes the task was very easy and both groups were performing at ceiling which would prevent any difference being detected. Secondly, at the higher set sizes when the task became more difficult and a difference in search behaviour could be noted, the individuals with ASD were still processing the distractor letter. This could have offset any increase in search efficiency.

The results presented here seem to suggest perceptual capacity differences between the groups. According to Lavie (1995), if the central task does not exhaust the perceptual capacity then additional information within the visual field would also be processed. A larger perceptual capacity in ASD would therefore result in more distractor processing at high levels of perceptual load - but without a reduction in task performance. This increased distractibility, therefore, could be conceptualised as a reflection of an increased ability rather than a 'deficit' - albeit with potentially detrimental effects in real-life situations.

Increased perceptual capacity could also underlie the superior performance seen on experimental paradigms such as visual search and Embedded Figures tasks. In both feature- and conjunctive-search tasks, participants with ASD were more efficient at detecting the location of a target within an array of non-target elements (O'Riordan et al., 2001), and on the Embedded Figures Task individuals with ASD 
are faster to identify the hidden target shape from within the picture (Jolliffe \& Baron-Cohen, 1997; Shah \& Frith, 1983). Increased perceptual capacity would allow more elements within the visual field to be processed in a parallel fashion, facilitating faster target location and therefore may underlie both these abilities.

Interestingly, the performance of the control group within this study differs slightly from results produced by previous studies. Maylor and Lavie, (1998); Huang-Pollock et al., (2002); and Lavie et al, (2003) have reported that for typically developed adults, the reduction in interference effects is seen between set sizes four and six. The control group within this study shows an earlier reduction in interference (between set sizes two and four). It is unclear why this difference in performance is seen given that similar procedures were used and participants across the studies were equivalent in age and IQ. There may have been some differences in stimulus presentation (for example, exact values for screen contrast, room lighting levels, exact colour of stimuli/background were not reported in previous studies) although it is unclear whether these factors could influence the perceptual load effect (Lavie \& de Fockert, 2003). Is it important to note, however, that all participants in this study (both ASD and control) performed the tasks under the same experimental conditions and therefore the difference between the two groups is still meaningful despite the shift in the absolute values seen for the control group.

This first study, therefore, offers evidence of increased perceptual capacity in ASD. The implications of this finding will be further discussed in chapter seven. 


\section{Chapter 4: Perceptual Load and Conscious Perception in ASD}

\section{Introduction}

In the previous chapter, evidence of increased perceptual capacity in ASD was presented. These conclusions were based on analyses of reaction time data which indicated that individuals with ASD were processing distractor elements at higher levels of perceptual load than control participants. Reaction time (RT) data, however, is an indirect measure of attention. While such measures can demonstrate whether non-target elements are being processed, and therefore affecting task performance, they cannot provide any information regarding whether the elements in question are actually consciously perceived. In the second experiment, I therefore explored whether an increase in perceptual load differentially affects conscious perception of irrelevant distractors in ASD.

Though the concepts of attention and consciousness are intrinsically linked, it is also possible to dissociate the two. Williams (1939) showed participants the very faint outline of a shape and asked them to state the first shape that entered their mind. They were also asked to indicate whether they had seen the shape clearly, had seen something but were not entirely sure which shape they had seen, or had seen nothing at all and therefore were making a pure guess regarding the identity of the shape. The results were greater than chance level - even for cases when the participant reported having seen nothing at all on the screen. In a more extreme example, it was demonstrated that while patients with a V1 lesion cannot consciously perceive, they can catch a ball and make correct guesses concerning visual stimuli that are at a greater level than chance (Weiskranz, Warrington, Sanders \& Marshall, 1974). The 
effect of distractors on RT data could therefore be attributed to unconscious processing of stimulus-response associations rather than conscious perception. With this is mind, Lavie and colleagues have recently begun to explore whether perceptual load modulates the conscious awareness of task-irrelevant stimuli. Cartwright-Finch and Lavie (2007) used an attentional blindness paradigm to assess the awareness of an unexpected stimulus presented on the last trial of an experiment. They presented participants with a series of visual displays, each containing a cross, and asked them to indicate which arm of the cross was longer (high load condition) or which arm of the cross was blue (low load condition). On the final trial, the critical stimulus (a faint shape outline) was also presented next to the cross. Their results demonstrated that awareness of the critical stimulus (indicated by post-test questioning) was linked to the perceptual load of the experimental task: with the rate of awareness significantly dropping (by approximately 40\%) in trials with a high level of perceptual load.

With a retrospective method of awareness such as this, however, inattentional blindness may not be the only reason that the critical stimulus was not reported. It is possible that the critical stimulus was perceived, but that the combination of the surprise question and the unexpected nature of the stimulus led to rapid forgetting (Wolfe, 1999) or weak signal generation (Barber \& Folkard, 1972).

To address this concern, MacDonald and Lavie (2008) employed a dual-task paradigm in which the conscious perception of an expected critical stimulus was measured online - thus negating the influence of memory limitations. Participants performed a central letter-search task while also looking out for a critical stimulus (a small grey figure) that they were told would appear in some trials. The perceptual load of the central task was manipulated to create a high and low load condition. 
MacDonald and Lavie calculated the detection sensitivity (d') and the response bias ( $\beta$ ) for both the high and low load conditions. In line with the predictions from the more indirect methods of their earlier experiments, they showed that the sensitivity to the critical stimulus was significantly reduced in the high perceptual load condition whereas the response bias was unchanged. This confirmed that conscious perception was indeed modulated by perceptual load.

The aim of the experiment reported here is to establish whether the differences between the two groups that were reported the previous chapter reflect the fact that individuals with ASD can consciously perceive more information at any one time. The conclusions made regarding increased perceptual capacity in ASD were based on indirect RT measures which, as discussed earlier, cannot lead to any definite assertions concerning conscious perception. At the higher set sizes, the lingering distractor interference effects in the ASD group may be solely due to the unconscious processing of stimulus-response associations or, conversely, the distractors may have been perceived at all set sizes - with any elimination of the interference effect at the highest set size being a result of post-perceptual response selection processes (Macdonald \& Lavie, 2008).

To resolve this issue, a dual-task paradigm was used to examine the sensitivity to a critical stimulus under four different levels of perceptual load. It was predicted that both the ASD and control individuals would show a reduction in sensitivity to the critical stimulus as the perceptual load of the central task increased. This reduction, however, would occur earlier for the control participants resulting in the ASD individuals showing greater sensitivity than control individuals at the higher levels of perceptual load. This would indicate that the findings from the previous experiment extend to conscious perception - and would be manifest as an increased 
awareness of the critical stimulus at higher levels of perceptual load on the dual-task paradigm.

\section{Experiment Two: Dual-task performance under perceptual load.}

\section{Participants}

Participants in this study were 16 adults with Asperger's Syndrome and 16 typical adults. The participants were diagnosed and recruited in the same manner as in the previous experiment. Two participants with ASD and two control participants were excluded from the sample due to high error rates. The remaining 14 in each group were matched for chronological age and non-verbal IQ using the matrix reasoning sub-scale from the Wechsler Abbreviated Scale for Intelligence (WASI) (Wechsler, 1999) (see table 4.1).

Table 4.1 Descriptive Statistics for each Group

\begin{tabular}{llcccc}
\hline Group & Statistic & $\begin{array}{c}\text { Age } \\
\text { (years: } \\
\text { months) }\end{array}$ & $\begin{array}{c}\text { WASI } \\
\text { vocabulary } \\
\text { subtest }\end{array}$ & $\begin{array}{c}\text { WASI } \\
\text { matrix } \\
\text { reasoning }\end{array}$ & $\begin{array}{c}\text { WASI } \\
\text { full scale IQ } \\
\text { (2 subtests) }\end{array}$ \\
\hline ASD $(\mathrm{n}=14)$ & mean & $24: 1$ & 65.2 & 55.9 & 119 \\
11 males & S.D & $4: 5$ & 6.7 & 9.6 & 13.4 \\
3 females & range & $18: 09-30: 11$ & $54-76$ & $39-67$ & $97-138$ \\
\hline Control (n=14) & mean & $25: 9$ & 66.9 & 56.0 & 121 \\
6 males & S.D. & $3: 0$ & 3.8 & 8.4 & 8.0 \\
8 females & range & $19: 8-30: 1$ & $60-71$ & $41-65$ & $109-131$ \\
\hline
\end{tabular}

Independent samples t-tests indicated that the groups did not differ on any of these measures (all p values $>0.3$ ). 


\section{Methodology}

\section{Apparatus and Stimuli}

Visual basic was used to create computer based stimuli that were presented on a custom built small form desktop computer and displayed on a ProLite 15" flat LCD screen (1280 x 1024 pixel resolution, 2ms response rate). Viewing distance was $60 \mathrm{~cm}$.

The task involved a dual-task paradigm that required participants to identify a target letter $(\mathrm{X}$ or $\mathrm{N})$ and then indicate the presence or absence of a meaningless small grey character (the critical stimulus (CS)) that was presented outside the ring.

Participants were presented with six letters that were placed, equally spaced, around the circumference of an imaginary circle with a radius of $1.7^{\circ}$ visual angles. In each trial, one of the letters was the target letter $(\mathrm{X}$ or $\mathrm{N})$ and, depending on the condition, the other ring positions were occupied by a non-target letter that was perceptually similar to the target $(\mathrm{Z}, \mathrm{H}, \mathrm{K}, \mathrm{Y}$ or $\mathrm{V}$ ) or a small letter $O$ (easy to distinguish from the target-letter). Target and similar non-target elements measured $0.6^{\circ} \times 0.6^{\circ}$ visual angles. The dissimilar non-target element $(O)$ measured $0.2^{\circ} \mathrm{x}$ $0.2^{\circ}$

The perceptual load of the task was manipulated by changing the number of similar non-target letters and $O$ s in the ring to create trials with set size of one (the target letter and five $O$ s in the ring), two (target, one similar non-target and four $O$ s in the ring), four (target, three similar non-target elements and two $O$ s) and six (target and five similar non-target letters).

In $50 \%$ of the trials the CS, a meaningless grey squiggle, measuring $0.3^{\circ} \mathrm{x}$ $0.3^{\circ}$ visual angles was presented, outside the ring of letters, in one of six positions that were arranged on the circumference of an imaginary circle with radius $5.4^{\circ}$. The 
six CS positions were such that each one lay on an imaginary line that passed through the centre of the circle and bisected two adjacent letter locations (see figures 4.1 and 4.2).

Figure 4.1 Examples of Experimental Stimuli

4.1a: Example of a high perceptual load trial with the CS present. In this case the set size is six and the target is $\mathrm{X}$.

4.1b: Example of a low perceptual load trial with a no CS present. In this case the set size is one and the target is $\mathrm{N}$.

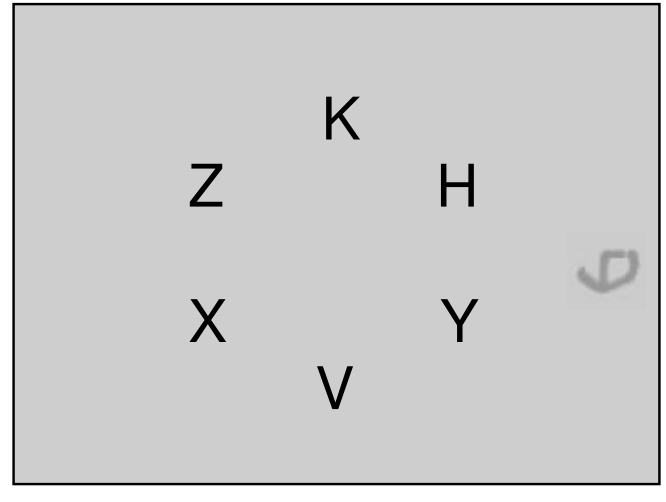

Figure 4.1a

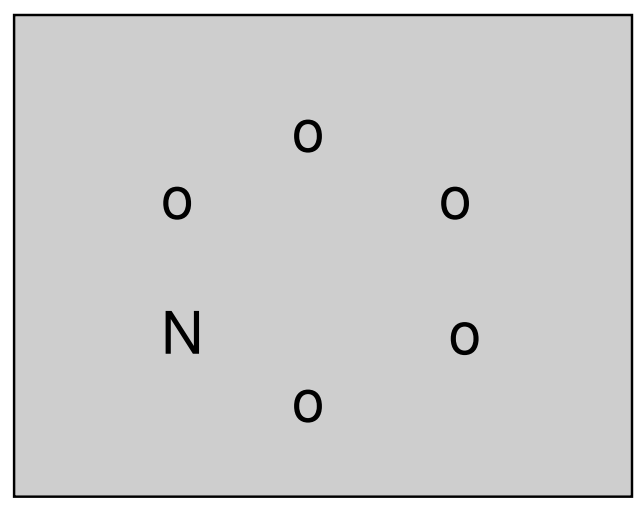

Figure $4.1 b$

Figure 4.2 The positions that the critical stimulus could occupy outside the ring of letters

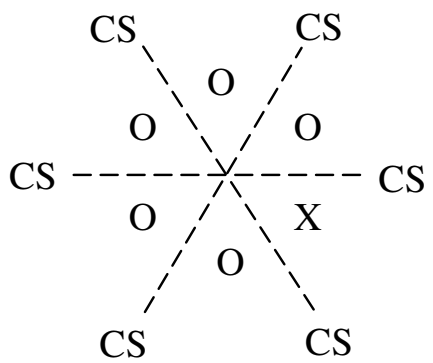


The location of the target, its proximity to the CS and, in set size 4, the target letter's location within the group of similar non-target elements (edge/middle) were counterbalanced across all the trials.

The letters in the ring were presented in black on a light grey background (Red Green Blue (RGB) values: 204,204,204) and the CS was a darker grey (RGB: $153,153,153)$. Following the presentation of the experimental stimulus, a black mesh pattern that covered the entire screen other than the central $9.5^{\circ}$ by $9.5^{\circ}$ square, was presented as a mask. The clear square in the centre was to ensure that the ring of letters was not masked.

72 trials of each set size were created. These were presented as four blocks of each set size, where each block contained 18 trials of the same set size. Participants were permitted to take short breaks between each block. Blocks were presented such that all 72 trials of any one set size were performed before moving on to four blocks of a different set size. The first four blocks presented to each participant contained trials of set size two, the presentation order of the other set sizes was randomly determined by the computer program.

A control block of 72 trials was also created where participants were told not to search for the target letter but only to indicate the presence or absence of the CS. 18 trials of each set size, $50 \%$ containing the CS, were used to create the control block.

\section{Procedure}

Following a fixation cross that was presented for $1000 \mathrm{~ms}$, the experimental display was presented for only $100 \mathrm{~ms}$ in order to preclude voluntary eye movement. 
The mask was then presented for $500 \mathrm{~ms}$, followed by a blank screen for $1.4 \mathrm{~s}$, a question mark for $100 \mathrm{~ms}$ and a final blank screen for 1.9 seconds (see figure 4.3). The duration of the two blank screen phases was such that there was a 2 -second response window for the letter identification and the CS detection tasks. Both these 2-second windows elapsed regardless of the response time. If no response was given within the time limit, the trial was recorded as 'time out' and classed as an error.

Participants were told that they would see a ring made up of letters in the centre of the screen and that one of those letters would be either an X or an N. They were told to press the $\mathrm{Z}$ key if an $\mathrm{X}$ was present and the $\mathrm{X}$ key if an $\mathrm{N}$ was present and to respond as quickly as they could after they saw the ring of letters. Participants were also told that they should look for a little grey squiggle that would appear outside the ring in some of the trials. They were instructed to wait until they saw the question mark and then indicate with a key-press ( $\mathrm{N}$ for present, $\mathrm{M}$ for absent) whether the CS was present or absent in that trial. Stickers were placed over the Z, X, $\mathrm{N}$ and $\mathrm{M}$ keys in order to clarify which key corresponded to which response.

Following a set of practice trials, the participants completed the 16 experimental blocks and were able to take breaks between blocks if required. No difference was noted in the number, or duration, of breaks taken by each group. An incorrect response on the letter detection task elicited a brief computer tone, and participants were informed that this indicated an error had been made. The accuracy and response times for each trial were recorded by the computer program and subsequent comparison of CS detection rates at the various levels set sizes allow the effect of perceptual load on dual-task performance to be ascertained. After completing the experimental trials, participants performed the control block in order to ensure that all the participants were able to detect the CS. This was vital to 
confirm that any failures to detect the CS during the experimental trials were due to the perceptual load of the central letter search task, and not an underlying inability to recognise or perceive the squiggle.

Figure 4.3 Time-course of experimental trials

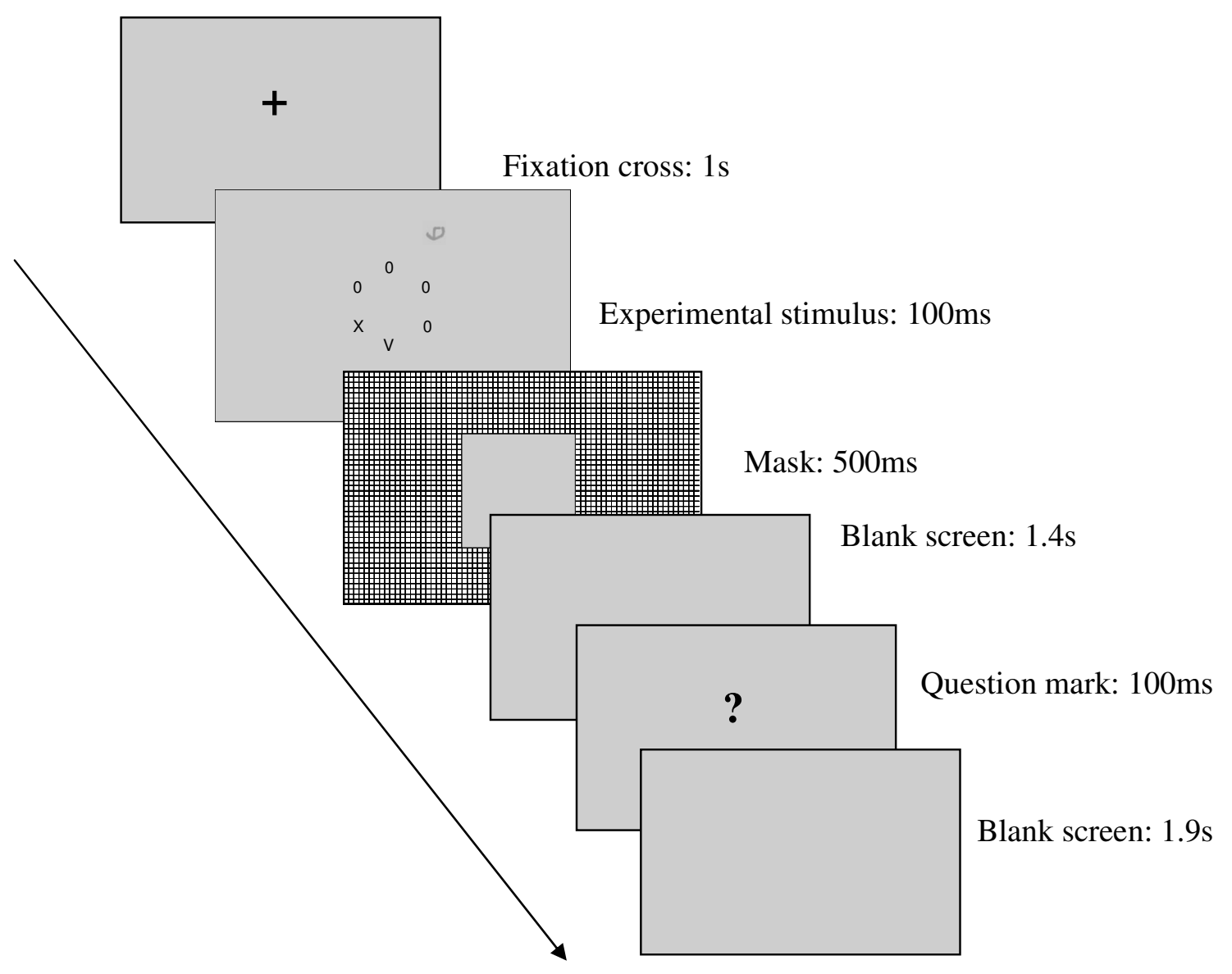

\section{Results}

\section{Letter Search}

All incorrect trials were excluded from further analyses and an analysis of variance (ANOVA) was performed on the median correct RTs with group (ASD vs. control) 
as the between-subjects factor and set size (one, two, four \& six) as the withinsubject factor (see table 4.2).

Table 4.2 Mean reaction times (RT) and standard deviation for each group at each set size

\section{Mean RT (Standard Deviation)}

Set size

ASD

Control

$\begin{array}{lllll}\mathbf{1} & 794 & (140) & 864 & (204) \\ \mathbf{4} & 845 & (226) & 823 & (177) \\ \mathbf{6} & 928 & (205) & 935 & (233) \\ & 990 & (242) & 966 & (225)\end{array}$

There was a significant main effect of set size indicating that participants were slower to respond to trials with higher set sizes $\left(F(3,78)=13.90, \mathrm{p}<0.001, \eta_{\mathrm{p}}{ }^{2}=\right.$ 1.00). There was no main effect of group $\left(F(1,26)=0.01, p=0.933, \eta_{p}{ }^{2}=0.05\right)$ which means that the reaction times were not significantly slower or faster in either group. This indicates that any differences between the groups are not due to a generalised reduction in processing speed within ASD. There was also no significant interaction between set size and group $\left(F(3,78)=1.35, \mathrm{p}=0.264, \eta_{\mathrm{p}}{ }^{2}=0.35\right)$.

\section{Letter search error analysis}

Analysis of the letter search error rates at each set size revealed a main effect

of set size $\left(F(3,78)=60.88, p<0.001, \eta_{p}^{2}=1.00\right)$. Inspection of the means indicated that this is a reflection of the increasing number of errors as perceptual load of the 
central task was increased (see table 4.3). There was no main effect of group ( $\mathrm{F}(1$, 26) $\left.=0.14, \mathrm{p}=0.708, \eta_{\mathrm{p}}{ }^{2}=0.07\right)-$ neither group made significantly more errors - and no significant interaction between group and set size $\left(\mathrm{F}(3,78)=0.18, \mathrm{p}=0.907, \eta_{\mathrm{p}}{ }^{2}=\right.$ $0.08)$.

Table 4.3 Mean error rates (\%) and standard deviation.

\section{Mean error rate (Standard Deviation)}

Set size

ASD

Control

\begin{tabular}{lllll}
\hline $\mathbf{1}$ & 3.6 & $(6.0)$ & 1.9 & $(3.3)$ \\
$\mathbf{2}$ & 5.7 & $(7.6)$ & 5.6 & $(6.0)$ \\
$\mathbf{4}$ & 16.5 & $(12.5)$ & 16.6 & $(7.4)$ \\
$\mathbf{6}$ & 22.3 & $(8.8)$ & 20.6 & $(8.2)$ \\
\hline
\end{tabular}

This main effect of set size in the RT and error analyses confirm that perceptual load had been effectively manipulated and that participants were engaging with the task in the way that was intended.

\section{CS Detection}

All trials with incorrect letter search responses were excluded from the analysis. The percentage detection rate, false alarm rate and sensitivity ( $d$ ') for each participant at each set size was calculated (see table 4.4 for group means). The $d$ ' measure takes into account both the percentage detection rate and the false alarm rate in order to give a true measure of how sensitive an individual is to the presence of the critical stimulus. 
Table 4.4 Mean Percentage CS detection rate, false alarm rate and d' (with standard deviation) for each group under each condition.

\begin{tabular}{lllllll}
\hline & \multicolumn{2}{l}{ Detection Rate (\%) } & \multicolumn{2}{c}{ False Alarm Rate (\%) } & \multicolumn{2}{c}{ d' } \\
Set size & ASD & Control & ASD & Control & ASD & Control \\
\hline $\mathbf{1}$ & 85.7 & 90.1 & 8.49 & 6.34 & 2.91 & 3.21 \\
& $(13.5)$ & $(11.8)$ & $(13.5)$ & $(6.16)$ & $(1.00)$ & $(0.92)$ \\
$\mathbf{2}$ & & & & & & \\
& 86.9 & 87.5 & 12.5 & 8.44 & 2.76 & 2.88 \\
& $(16.8)$ & $(13.6)$ & $(13.3)$ & $(6.00)$ & $(1.08)$ & $(0.82)$ \\
$\mathbf{4}$ & & & & & & \\
& 89.7 & 81.3 & 12.0 & 14.3 & 2.90 & 2.32 \\
& $(14.5)$ & $(18.2)$ & $(12.3)$ & $(13.2)$ & $(0.75)$ & $(0.81)$ \\
$\mathbf{6}$ & 87.2 & 70.2 & 15.8 & 19.7 & 2.62 & 1.74 \\
& $(15.5)$ & $(24.5)$ & $(17.0)$ & $(17.2)$ & $(0.94)$ & $(1.22)$ \\
& & & & & & \\
\hline
\end{tabular}

\section{Percentage Detection}

A repeated measures ANOVA on the percentage detection rate indicated that there was a significant main effect of set size $\left(F(3,78)=3.92, p=0.012, \eta_{p}{ }^{2}=0.81\right)$, reflecting the fact that the CS was detected less often as the perceptual load of the central task increased. There was no significant main effect of group $(\mathrm{F}(1,26)=1.03$, $\left.\mathrm{p}=0.319, \eta_{\mathrm{p}}{ }^{2}=0.17\right)$ - neither group showed an overall difference in the rate of CS detection. There was, however, a significant interaction between group and set size $\left(\mathrm{F}(3,78)=5.10, \mathrm{p}=0.003, \eta_{\mathrm{p}}^{2}=0.91\right)$ suggesting that the two groups were showing a different pattern of sensitivity change across the various set sizes. The nature of this difference can be seen in figure 4.4. 
Figure 4.4 Graph of percentage CS detection

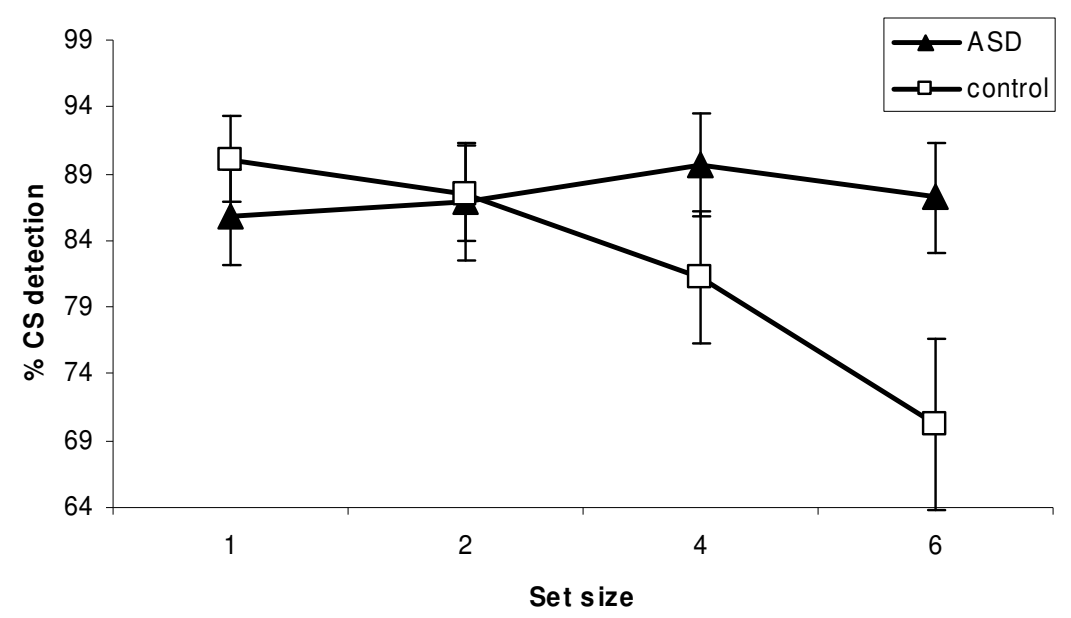

Post-hoc ANOVA for each group demonstrated that this interaction was a result of a greater drop in percentage detection rate in the control group as the set size increased. In the ASD group there was no significant difference between the detection rates at the different set sizes $\left(\mathrm{F}(3,39)=0.48, \mathrm{p}=0.698, \eta_{\mathrm{p}}{ }^{2}=0.14\right)$ whereas in the control group there was a significant effect of set size on the percentage of CS detection $\left(\left(\mathrm{F}(3,39)=6.41, \mathrm{p}=0.001, \eta_{\mathrm{p}}{ }^{2}=0.95\right)\right.$. T-tests showed that there was also a significant difference between the detection rates of the two groups at set size six $(\mathrm{t}(26)=2.19 \mathrm{p}=0.037)$.

\section{False alarm rate}

A repeated measures ANOVA indicated that the main effect of set size was significant $\left(F(3,78)=5.84, p=0.001, \eta_{p}{ }^{2}=0.94\right)$, with the false alarm rate increasing with set size (see figure 4.5). This was expected, as the task becomes more difficult and therefore necessitates a greater level of guessing. Neither the interaction between group and set size $\left(\mathrm{F}(3,78)=1.06, \mathrm{p}=0.089, \eta_{\mathrm{p}}{ }^{2}=0.28\right)$ nor the main effect of group was significant $\left(\mathrm{F}(1,26)=1.42, \mathrm{p}=0.245, \eta_{\mathrm{p}}{ }^{2}=0.21\right)$. 
Figure 4.5 Graph of false alarm rates.

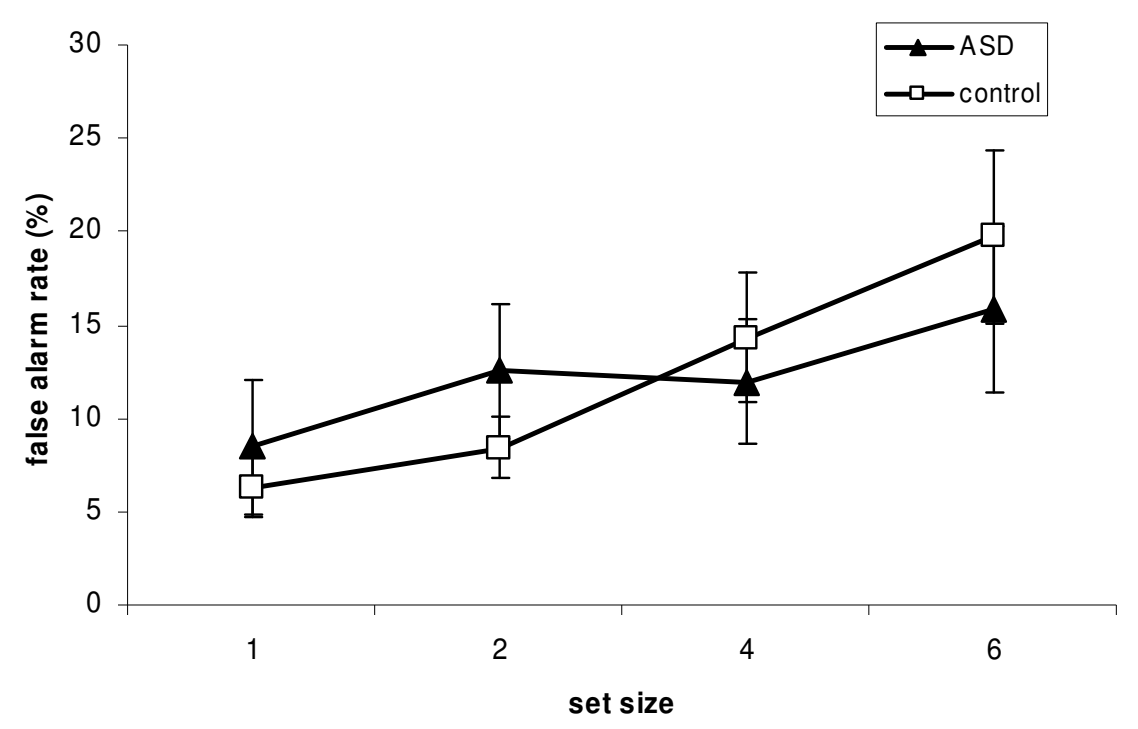

\section{Detection sensitivity}

The d' measure gives a more accurate reflection of task performance as it takes into account both the percentage detection and false alarm rates. Results of the repeated measures ANOVA on the $d$ ' values for each participant at each set size indicated a significant main effect of set size $\left(F(3,78)=9.25, \mathrm{p}<0.001, \eta_{\mathrm{p}}{ }^{2}=0.99\right)$. Inspection of the means showed that sensitivity was lower at the higher set sizes. There was also a significant interaction between group and set size $(F(3,72)=5.17, p$ $\left.=0.003, \eta_{\mathrm{p}}{ }^{2}=0.91\right)$ which suggests that the sensitivity is changing differently for the two groups as the perceptual load increased (see figure 4.6). There was no significant main effect of group $\left(F(1,26)=0.81, p=0.377, \eta_{p}^{2}=0.14\right)-$ there was no overall difference in the sensitivity levels of the two groups. 
Figure 4.6 Graph of detection sensitivity.

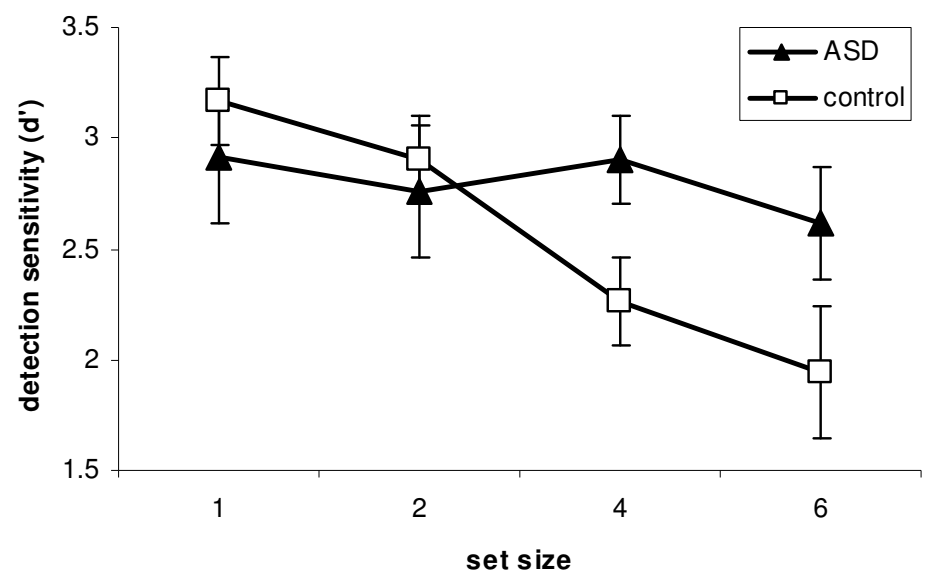

ANOVA within each group showed that there was no effect of set size on the sensitivity of the ASD group $\left(F(3,39)=0.86, p=0.470, \eta_{p}{ }^{2}=0.22\right)$ but that there was a significant main effect of set size in the control group $(\mathrm{F}(3,39)=10.80, \mathrm{p}<$ $\left.0.001, \eta_{\mathrm{p}}^{2}=0.99\right)$.

Post-hoc t-tests indicated that while there was no difference between the groups at set size one and two (both p values $>0.4$ ) there was a significant difference between the d' values of the two groups at set size $6(t(26)=2.14 \mathrm{p}=0.042)$ and the difference at set size four was close to significance $(\mathrm{t}(26)=1.98 \mathrm{p}=0.058)$.

Paired t-tests comparing the means for each set size within the groups showed that for the ASD individuals there was no significant difference between the d' values across the set sizes (all p values $>0.2$ ). Within the control group, however, there was a significant difference between the d' values at set size two and four ( $t$ $(13)=2.81 \mathrm{p}=0.015)$. 


\section{Response Bias}

The response bias $(\beta)$ was also calculated for each participant at each set size:

$$
\beta=\left\{\frac{Z_{S \mid n}^{2}-Z_{S \mid s}^{2}}{2}\right\}
$$

where $\mathrm{Z}_{\text {Sls }}^{2}=$ normal value corresponding to proportion of correct ' $\mathrm{CS}$ present' responses, and $\mathrm{Z}_{\text {SIn }}^{2}=$ normal value corresponding to proportion of incorrect ' $\mathrm{CS}$ present' responses.

The response bias did not change at the various levels of perceptual load $\left(\mathrm{F}(3,78)=0.57, \mathrm{p}=0.639, \eta_{\mathrm{p}}{ }^{2}=0.16\right)$ and did not differ between the groups $(\mathrm{F}(1,26)$ $\left.=0.19, \mathrm{p}=0.664, \eta_{\mathrm{p}}{ }^{2}=0.07\right)$. The interaction between group and set size was also non-significant: $\mathrm{F}(3,78)=1.25, \mathrm{p}=0.297, \eta_{\mathrm{p}}{ }^{2}=0.32$ (see table 4.5 for means).

Table 4.5 Mean response bias $(\beta)$ for each group at each set size.

\section{Response Bias $(\beta)$}

Set size $1 \quad$ Set size $2 \quad$ Set size $4 \quad$ Set size 6

$\begin{array}{lllll}\text { ASD } & 2.5(2.5) & 1.6(1.5) & 1.7(2.5) & 1.3(1.1) \\ \text { Control } & 1.5(1.2) & 1.6(1.6) & 1.6(1.4) & 1.9(2.5)\end{array}$

\section{Error Analysis}

Analysis of the CS detection error rates revealed a main effect of set size $\left(\mathrm{F}(3,78)=15.46, \mathrm{p}<0.001, \eta_{\mathrm{p}}^{2}=1.00\right)$. Inspection of the means indicated that this is a reflection of the increasing number of errors as perceptual load of the central task was increased (see table 4.6). There was no main effect of group $(F(1,26)=0.03$, $\left.\mathrm{p}=0.859, \eta_{\mathrm{p}}^{2}=0.05\right)-$ neither group made significantly more errors - and no 
significant interaction between group and set size $\left(F(3,78)=2.28, \mathrm{p}=0.086, \eta_{\mathrm{p}}{ }^{2}=\right.$ $0.56)$.

Table 4.6 Mean error rates (\%) and standard deviation for each group at each set size.

\begin{tabular}{lllll}
\hline & \multicolumn{4}{l}{ Mean error rate (Standard Deviation) } \\
Set size & \multicolumn{2}{c}{ ASD } & \multicolumn{2}{c}{ Control } \\
\hline $\mathbf{1}$ & 11.5 & $(9.6)$ & 7.3 & $(7.5)$ \\
$\mathbf{2}$ & 13.4 & $(13.0)$ & 9.2 & $(6.0)$ \\
$\mathbf{4}$ & 16.4 & $(14.6)$ & 20.4 & $(7.4)$ \\
$\mathbf{6}$ & 22.5 & $(15.2)$ & 29.8 & $(8.2)$ \\
\hline
\end{tabular}

\section{Control Block}

All the participants achieved a correct response rate of over $84 \%$ on the control block. Analysis of variance indicated that there were no significant effects of set size on the detection rates, and no group differences (all $\mathrm{p}$ values $>0.2$ ). This confirms that any differences seen in the pattern of CS detection under various levels of perceptual load is not due to an underlying inability to recognise or respond to the CS.

\section{Discussion}

The results presented here provide preliminary evidence for the fact that ASD individuals show altered performance on a dual-task paradigm under various levels of perceptual load. 
In line with MacDonald and Lavie (2008), the control individuals in this study demonstrated that their ability to detect the irrelevant stimulus was dependant on the perceptual load of the central search task. Both the percentage detection rate and the sensitivity (d') dropped as the perceptual load increased while response bias ( $\beta$ ) remained constant (implying that the drop in detection rates is not due to a shift in response criteria). These results replicate MacDonald and Lavie's findings regarding the modulation of conscious perception by perceptual load. In their study, two different load conditions were used (high and low, set size 1 and 6 respectively) and the results are very similar to those found for the typical group in my experiment. In the low load condition, MacDonald and Lavie recorded a detection rate of $92 \%$, a false alarm rate of $5 \%$ and a d' of 3.38. Within my study, the detection rate for the control group was $90 \%$, the false alarm rate was $6.3 \%$ and d' was 3.21 .

The pattern of results in the ASD group, however, appears to be different. Both the percentage detection rate and the perceptual sensitivity seem to remain constant as the level of perceptual load increases; individuals detected the presence of the CS more often than the control individuals at the higher levels of perceptual load (set size four and six).

These results varied slightly from the original predictions. It was predicted that the ASD individuals would show higher levels of CS detection at the higher set sizes - something that is indeed seen in the data. It was also predicted, however, that both groups would show an effect of perceptual load on the ability to detect the CS. This variation is not seen for the ASD group: where d' and percentage detection does not change across the various set sizes. These findings and possible explanations will be discussed more fully in the chapter discussion. 


\section{Experiment Three: Dual-task performance under perceptual load - mixed}

trials.

One potentially problematic issue that may have arisen within the previous experiment concerns the use of a blocked design: where each block only contained trials of the same set size. It is possible that an experimental design such as this could allow the use of different strategies for high and low perceptual load blocks. These strategy differences, rather than the level of perceptual load, could be responsible for the effects seen on percentage detection and d' values as the load of the central task was increased. This issue has been addressed in a number of studies (BrandD'Abrescia \& Lavie, 2007; Cartwright-Finch \& Lavie, 2007; Macdonald \& Lavie, 2008) where the procedure is modified to create experimental blocks containing all trial types randomly intermixed.

In order to explore the possibility of strategy effects within the dual-task data presented above, a second version of the task was created - with all trial types mixed evenly across the blocks. It was predicted, in line with the findings of MacDonald and Lavie (2008), that this modification would not alter the results obtained: as the perceptual load of the central task increased, the control group would show a drop in sensitivity while the ASD group would show no change.

\section{Participants}

The participants were the same 28 subjects that had previously taken part in the blocked experiment. 


\section{Methods}

The stimuli and procedures were the same as those of experiment two, except that the blocks contained trials of all four set sizes randomly intermixed. The trials were divided into four blocks of 72 trials, containing 18 trials of each set size that were presented in a random order. Each of these four blocks was then further divided into four smaller blocks. As in experiment two, participants were permitted to take a break after each block.

\section{Results}

\section{Letter Search}

All incorrect trials were excluded from further analyses and an analysis of variance (ANOVA) was performed on the median correct RTs with group (ASD vs. control) as the between-subjects factor and set size (one, two, four $\&$ six) as the within-subjects factor (see table 4.7).

Table 4.7 Mean reaction times (RT) and standard deviation for each group at each set size.

Mean RT (Standard Deviation) (ms)

Set size

ASD

Control

\begin{tabular}{lllll}
\hline $\mathbf{1}$ & 715 & $(195)$ & 694 & $(185)$ \\
$\mathbf{2}$ & 779 & $(216)$ & 756 & $(196)$ \\
$\mathbf{4}$ & 885 & $(246)$ & 883 & $(242)$ \\
$\mathbf{6}$ & 919 & $(243)$ & 924 & $(246)$ \\
\hline
\end{tabular}


As in the previous experiment, there was a significant main effect of set size indicating that participants were slower to respond to trials with higher set sizes $\left(F(3,78)=69.49, \mathrm{p}<0.001, \eta_{\mathrm{p}}{ }^{2}=1.00\right)$. There was no main effect of group $(\mathrm{F}(1,26)$ $\left.=0.02, \mathrm{p}=0.900, \eta_{\mathrm{p}}{ }^{2}=0.05\right)$ which means that the reaction times were not significantly slower or faster in either group. There was also no significant interaction between set size and group $\left(\mathrm{F}(3,78)=0.32, \mathrm{p}=0.814, \eta_{\mathrm{p}}{ }^{2}=0.11\right)$.

Analysis of the letter search error rates at each set size revealed a main effect of set size $\left(F(3,78)=80.02, p<0.001, \eta_{p}^{2}=0.76\right)$. The number of errors increased with the perceptual load of the central task (see table 4.8). There was no main effect of group $\left(\mathrm{F}(1,26)=0.67, \mathrm{p}=0.420, \eta_{\mathrm{p}}{ }^{2}=0.03\right)$ and no significant interaction between group and set size $\left(\mathrm{F}(3,78)=0.23, \mathrm{p}=0.878, \eta_{\mathrm{p}}{ }^{2}=0.01\right)$.

Table 4.8 Mean error rates (\%) and standard deviation for each group at each set siz

\begin{tabular}{lllll}
\hline Set size & \multicolumn{2}{c}{$\begin{array}{l}\text { Mean error rate } \\
\text { ASD }\end{array}$} & $\begin{array}{c}\text { (Standard Deviation) }(\%) \\
\text { Control }\end{array}$ \\
\hline $\mathbf{1}$ & 4.3 & $(8.6)$ & 2.0 & $(2.5)$ \\
$\mathbf{2}$ & 6.1 & $(8.2)$ & 3.0 & $(2.3)$ \\
$\mathbf{4}$ & 14.3 & $(15.5)$ & 12.9 & $(6.3)$ \\
$\mathbf{6}$ & 24.1 & $(14.0)$ & 20.6 & $(9.2)$ \\
\hline
\end{tabular}




\section{CS Detection}

All trials with incorrect letter search responses were excluded from the analysis. The percentage detection rate, false alarm rate and $d$ ' for each participant at each set size was calculated (see table 4.9 for group means).

Table 4.9 Mean Percentage CS detection rate, false alarm rate and d'(and standard deviation) for each group under each condition.

\begin{tabular}{|c|c|c|c|c|c|c|}
\hline \multirow[b]{2}{*}{ Set size } & \multicolumn{2}{|c|}{$\begin{array}{c}\text { Detection Rate (\%) } \\
\text { (S.D.) }\end{array}$} & \multicolumn{2}{|c|}{$\begin{array}{l}\text { False Alarm Rate (\%) } \\
\text { (S.D.) }\end{array}$} & \multicolumn{2}{|c|}{$\begin{array}{c}\text { d' } \\
\text { (S.D.) }\end{array}$} \\
\hline & $\mathrm{ASD}$ & Control & ASD & Control & $\mathrm{ASD}$ & Control \\
\hline 1 & $\begin{array}{l}89.7 \\
(17.0)\end{array}$ & $\begin{array}{l}93.9 \\
(9.2)\end{array}$ & $\begin{array}{l}7.57 \\
(11.2)\end{array}$ & $\begin{array}{l}8.74 \\
(9.1)\end{array}$ & $\begin{array}{l}3.33 \\
(1.2)\end{array}$ & $\begin{array}{l}3.39 \\
(0.9)\end{array}$ \\
\hline 2 & $\begin{array}{l}89.0 \\
(11.5)\end{array}$ & $\begin{array}{l}90.1 \\
(11.9)\end{array}$ & $\begin{array}{r}7.47 \\
(7.3)\end{array}$ & $\begin{array}{l}10.4 \\
(10.3)\end{array}$ & $\begin{array}{l}3.07 \\
(0.9)\end{array}$ & $\begin{array}{l}3.10 \\
(0.9)\end{array}$ \\
\hline 4 & $\begin{array}{l}85.5 \\
(21.5)\end{array}$ & $\begin{array}{l}90.7 \\
(12.9)\end{array}$ & $\begin{array}{r}8.45 \\
(8.5)\end{array}$ & $\begin{array}{l}11.9 \\
(12.2)\end{array}$ & $\begin{array}{l}3.00 \\
(1.0)\end{array}$ & $\begin{array}{r}2.94 \\
(0.9)\end{array}$ \\
\hline 6 & $\begin{array}{l}85.4 \\
(21.3)\end{array}$ & $\begin{array}{l}85.9 \\
(14.3)\end{array}$ & $\begin{array}{c}8.53 \\
(12.9)\end{array}$ & $\begin{array}{l}10.9 \\
(10.1)\end{array}$ & $\begin{array}{l}2.90 \\
(1.2)\end{array}$ & $\begin{array}{l}2.67 \\
(0.8)\end{array}$ \\
\hline
\end{tabular}

\section{Percentage Detection}

A repeated measures ANOVA demonstrated that there was a significant main effect of set size $\left(F(3,78)=3.46, p=0.020, \eta_{p}{ }^{2}=0.76\right)$. The detection rate fell as the perceptual load of the central task was increased. This is in line with the previous experiment, and suggests that mixing the trial types has not eliminated the effect of perceptual load on the level of CS detection, though the effect has been somewhat 
reduced (a reduction in d' of 0.72 compared to a reduction of 1.47 in experiment two) .

In this second experiment however, the interaction between group and set size was not significant $\left(F(3,78)=0.70, \mathrm{p}=0.554, \eta_{\mathrm{p}}^{2}=0.19\right)$.

The main effect of group was also not significant $\left(\mathrm{F}(1,26)=0.26, \mathrm{p}=0.319, \eta_{\mathrm{p}}{ }^{2}=\right.$ $0.08)$.

Figure 4.7 Graph of CS detection rates.

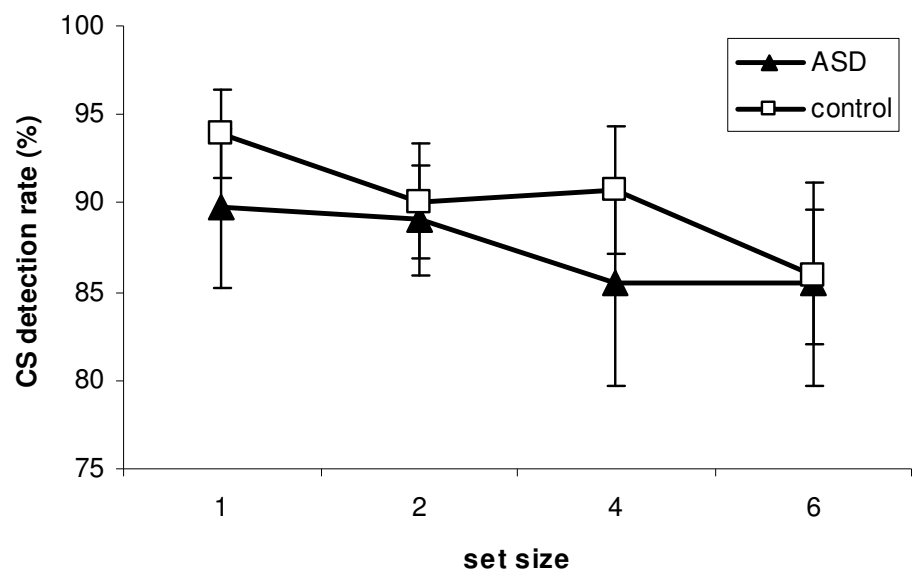

Inspection of the graph indicates that, although not significantly different, the CS detection rates for the control group were consistently higher than for the ASD group - and were higher than the results for the corresponding set sizes in the previous experiment. It seems to be this shift that has led to the elimination of the significant interaction between group and set size. However, this increased CS detection rate cannot be interpreted as an increase in performance without first taking into account the number of false alarm responses made by each group. Increased performance is only true if the number of hits (CS detection rate) rises without a concurrent rise in false alarm rates. 


\section{False alarm rate}

There was no significant main effect of set size $(F(3,78)=1.25, p=0.296$, $\left.\eta_{\mathrm{p}}{ }^{2}=0.32\right)$, main effect of group $\left(\mathrm{F}(1,26)=0.28, \mathrm{p}=0.599, \eta_{\mathrm{p}}{ }^{2}=0.08\right)$ or interaction between the two $\left(\mathrm{F}(3,78)=0.50, \mathrm{p}=0.686, \eta_{\mathrm{p}}{ }^{2}=0.15\right)$. Inspection of the graph, however, shows a generally higher level of false alarm responses in the control group (see figure 4.8). Given this trend, it is more appropriate to consider the d' values (which take into account both detection and false alarm rates) when assessing any difference in behaviour between the two groups.

Figure 4.8 Graph of false alarm rates of each group under each condition.

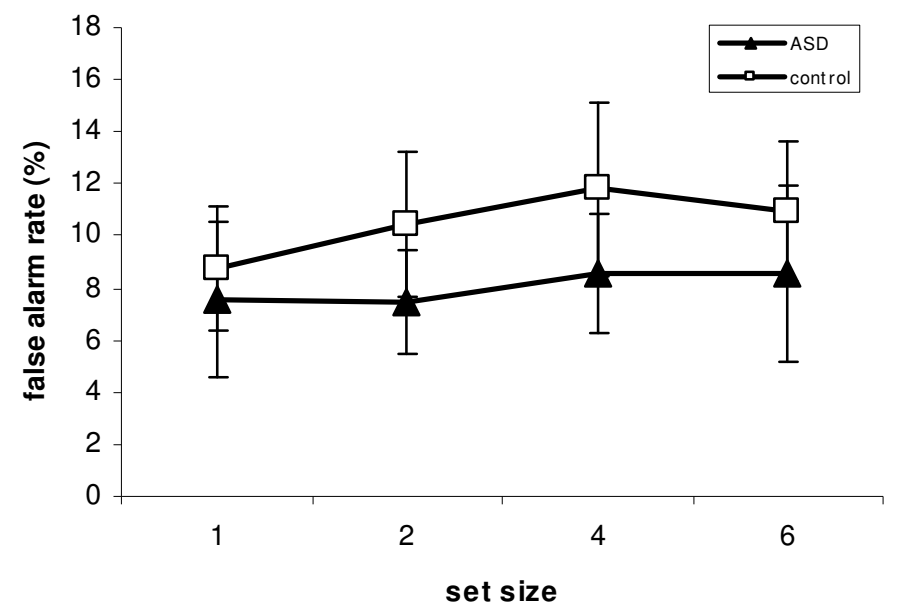

\section{Detection sensitivity (d')}

The main effect of set size was significant $\left(F(3,78)=6.74, p<0.001, \eta_{p}{ }^{2}=\right.$ 0.97) indicating that the sensitivity to the CS dropped as the perceptual load of the central task increased. There was no significant main effect of group $(\mathrm{F}(1,26)=0.06$, $\left.\mathrm{p}=0.802, \eta_{\mathrm{p}}^{2}=0.06\right)$. Both these findings are consistent with experiment two. 
However, the significant interaction between group and set size was no longer there $\left(\mathrm{F}(3,78)=0.53, \mathrm{p}=0.663, \eta_{\mathrm{p}}^{2}=0.15\right)($ see figure 4.9$)$.

Figure 4.9 Graph of detection sensitivity to the CS for each group at each set size

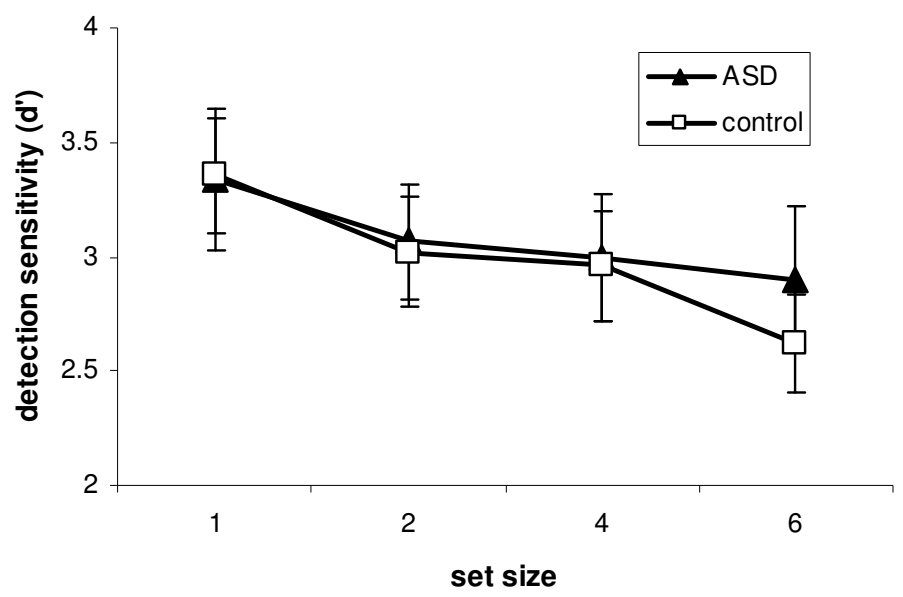

Following the a priori predictions that were made regarding this experiment, one way ANOVA was carried out on each group separately to examine the effect of perceptual load on d'. These analyses showed that there was only a significant effect of set size for the typical group $\left(\mathrm{F}(3,39)=4.79, \mathrm{p}=0.006, \eta_{\mathrm{p}}{ }^{2}=0.87\right)$ and not for the $\operatorname{ASD}$ group $\left(F(3,39)=2.22, \mathrm{p}=0.102, \eta_{\mathrm{p}}^{2}=0.52\right)$.

\section{Response Bias}

The mean response bias was calculated for each group under each condition. The response bias did not change at the various levels of perceptual load $(\mathrm{F}(3,78)=$ $\left.0.95, \mathrm{p}=0.420, \eta_{\mathrm{p}}{ }^{2}=0.04\right)$ and did not differ between the groups $(\mathrm{F}(1,26)=0.47, \mathrm{p}$ $\left.=0.500, \eta_{\mathrm{p}}^{2}=0.02\right)$. The interaction between group and set size was also nonsignificant: $F(3,78)=1.12, p=0.345, \eta_{p}^{2}=0.04$ (see table 4.10 for means). 
Table 4.10 Mean response bias $(\beta)$ and standard deviation for each group

\begin{tabular}{lcccc}
\hline & \multicolumn{4}{c}{ Response Bias (S.D) } \\
Set size 1 & Set size 2 & Set size 4 & Set size 6 \\
\hline \multirow{2}{*}{ Control } & $1.7(2.7)$ & $1.7(1.4)$ & $1.9(2.6)$ & $1.8(2.2)$ \\
& $0.9(0.8)$ & $1.9(2.9)$ & $1.1(1.1)$ & $1.5(1.3)$ \\
\hline
\end{tabular}

Error Analysis

Analysis of the CS detection error rates revealed a main effect of set size $\left(\mathrm{F}(3,78)=11.98, \mathrm{p}<0.001, \eta_{\mathrm{p}}{ }^{2}=0.32\right)$. This main effect is a reflection of the increasing number of errors as perceptual load of the central task was increased (see table 4.11). There was no main effect of group $\left(F(1,26)=0.14, p=0.715, \eta_{p}^{2}=0.01\right)$ and no significant interaction between group and set size $(\mathrm{F}(3,78)=0.20, \mathrm{p}=0.898$, $\left.\eta_{\mathrm{p}}^{2}=0.01\right)$

Table 4.11 Mean error rates (\%) and standard deviation for each group

\begin{tabular}{lllll}
\hline Set size & \multicolumn{2}{l}{$\begin{array}{l}\text { Mean error rate } \\
\text { ASD }\end{array}$} & $\begin{array}{c}\text { (Standard Deviation) } \\
\text { Control }\end{array}$ \\
\hline $\mathbf{1}$ & 9.02 & $(12.2)$ & 7.89 & $(8.13)$ \\
$\mathbf{2}$ & 9.94 & $(7.70)$ & 9.70 & $(8.04)$ \\
$\mathbf{4}$ & 15.1 & $(16.6)$ & 12.8 & $(10.8)$ \\
$\mathbf{6}$ & 19.5 & $(20.7)$ & 16.8 & $(12.0)$ \\
\hline
\end{tabular}




\section{Discussion}

The data from experiment three demonstrate that the pattern of behaviour of the two groups is no longer significantly different when the trial types are presented in mixed blocks. Post-hoc one way ANOVA results showed that only the control group is showing an effect of perceptual load on detection sensitivity and hence, as in

experiment two, that the two groups are showing differing responses. The effect of perceptual load on detection sensitivity in the control group, however, was significantly reduced in comparison to the reduction seen in response to high levels of load in the blocked procedure of experiment two. This is in line with the results reported in Macdonald and Lavie (2008) for the mixed trial paradigm. In my study, this reduction means the effect is no longer sufficient to produce a significant interaction in the repeated measures ANOVA. Consequently, it seems that changing the method of trial presentation has affected the task performance in the typical group.

Given that the modification to the experimental procedure was made in an attempt to rule out any effect of strategy differences that may arise in the blocked design, it is possible that it was strategy differences which were leading to the difference between the two groups in experiment two. To investigate this, a further analysis was carried out - adding experiment type as an additional within-subject factor in the ANOVA. This will establish in what way the presentation methodology is altering task performance and will help elucidate possible strategy influences. 


\section{Comparing the blocked and mixed trial procedures}

A repeated measures ANOVA was carried out on the detection rate, false alarm rate, detection sensitivity and error rate data from each experiment - with set size (1/2/4/6) and experiment (mixed/blocked) as within-subject factors and group as the between-subject factor.

\section{Comparison of search task $R T$ data in experiment two and three}

When the data from the two experiments were combined, ANOVA results indicated that there was a significant main effect of set size $(F(3,78)=38.03$, p < $\left.0.001, \eta_{\mathrm{p}}{ }^{2}=0.59\right)$. Inspection of the means indicated that as the set size increased, the reaction times also increased. There was also a significant main effect of experiment $\left(\mathrm{F}(1,26)=50.84, \mathrm{p}<0.001, \eta_{\mathrm{p}}{ }^{2}=0.66\right):$ the overall RTs are quicker when the trial types were presented in mixed blocks (experiment three).

The interactions between set size and group, experiment and group, set size and experiment and the three way interaction were not significant (all p values > $0.05)$ and there was no significant main effect of group $(F(1,26)=0.03, p=0.867$, $\left.\eta_{\mathrm{p}}^{2}<0.01\right)$

\section{Comparison of $R T$ error rates in experiment two and 3.}

There was a significant main effect of set size $(\mathrm{F}(3,78)=102.88, \mathrm{p}<0.001$, $\left.\eta_{\mathrm{p}}^{2}=0.80\right)$ and the main effect of experiment was just non-significant $(\mathrm{F}(1,26)=$ $\left.3.96, p=0.057, \eta_{p}{ }^{2}=0.13\right)$. The number of search task errors increased with set size and the number of errors made in the mixed experiment was lower than in the blocked design. The interaction between experiment and set size was also significant 
$\left(\mathrm{F}(3,78)=3.33, \mathrm{p}=0.024, \eta_{\mathrm{p}}{ }^{2}=0.11\right)$. The main effect of group and all other interactions were non-significant (all p values $>0.05$ ).

\section{Comparison of detection rates in experiment two and 3.}

There was a significant main effect of set size $\left(F(3,78)=5.47, p=0.002, \eta_{p}{ }^{2}\right.$ $=0.17)$ and a significant interaction between set size and group $(\mathrm{F}(3,78)=3.39, \mathrm{p}<$ $0.022, \eta_{\mathrm{p}}{ }^{2}=0.12$ ). This reflects the results seen in the previous analyses where the detection rate dropped as the set size was increased, and the fact that the perceptual load of the task appears to have less impact on the detection rates of the ASD group. There was a main effect of experiment $\left(F(1,26)=21.72, \mathrm{p}<0.001, \eta_{\mathrm{p}}{ }^{2}=0.46\right)$ with the detection rate appearing higher in the mixed trial experiment. The interaction between experiment and group is significant $\left(F(1,26)=6.63, p=0.016, \eta_{p}{ }^{2}=0.20\right)$ and the three way interaction between set size, group and experiment was also significant $\left(\mathrm{F}(3,78)=4.32, \mathrm{p}=0.007, \eta_{\mathrm{p}}{ }^{2}=0.14\right)$. Inspection of the means suggests that the experiment-group interaction is significant due the typical group showing higher detection rates in experiment three than they do in experiment two. The detection rates for the ASD group are similar in both experiments. This discrepancy between the two groups may well be due to a ceiling effect: the ASD group were already achieving very high detection rates in experiment two and therefore there was no potential for an increase of the same magnitude. The three way interaction reflects both this difference and the fact that in the mixed experiment, the ASD group showed slightly more effect of perceptual load on detection rates than they did in the blocked experiment. 
There was no overall effect of group $\left(F(1,26)=0.15, p=0.706, \eta_{p}^{2}=0.01\right)$ and no interaction between set size and experiment $\left(\mathrm{F}(3,78)=1.13, \mathrm{p}=0.342, \eta_{\mathrm{p}}{ }^{2}=\right.$ 0.04).

As previously explained, it is also important to take the false alarm rates into account in order to truly assess the task performance on each experiment.

\section{Comparison of false alarm rates in experiment two and 3.}

There was a main effect of set size $\left(F(3,78)=6.07, \mathrm{p}=0.001, \eta_{\mathrm{p}}{ }^{2}=0.19\right)$ and a main effect of experiment $\left(F(1,26)=9.61, p=0.005, \eta_{p}{ }^{2}=0.27\right)$. The interaction between the two was also significant $\left(F(3,78)=3.63, \mathrm{p}=0.016, \eta_{\mathrm{p}}{ }^{2}=0.12\right)$. Inspection of the means indicated that the false alarm rates increased with set size and were higher in the blocked experiment. There was less impact of set size on the false alarm rates in the mixed experiment. The main effect of group and all other interactions were not significant (all p values $>0.05$ ).

\section{Comparison of detection sensitivity in experiment two and 3.}

There was no significant main effect of group $\left(F(1,26)=0.07, p=0.796, \eta_{p}^{2}\right.$ $=0.07)$. Overall, neither group was more sensitive to the CS. There was a significant main effect of set size $\left(\mathrm{F}(3,78)=13.86, \mathrm{p}<0.001, \eta_{\mathrm{p}}{ }^{2}=0.35\right)$, a significant interaction between set size and group $\left(\mathrm{F}(3,78)=3.82, \mathrm{p}=0.013, \eta_{\mathrm{p}}{ }^{2}=0.13\right)$ and a significant main effect of experiment $\left(F(1,26)=30.16, \mathrm{p}<0.001, \eta_{\mathrm{p}}{ }^{2}=0.54\right)$. These results reflect the fact that d' dropped as the perceptual load of the central task increased, d' was lower in the blocked design and that the d' levels dropped more for the control group than the ASD group as the set size was increased. The interaction 
between experiment and group was just not significant $\left(F(1,26)=4.09, p=0.053, \eta_{p}^{2}\right.$ $=0.14)$ and there was no significant interaction between set size and experiment $\left(\mathrm{F}(3,78)=1.79, \mathrm{p}=0.156, \eta_{\mathrm{p}}^{2}=0.06\right)$.

There was a significant three way interaction between set size, experiment and group $\left(\mathrm{F}(3,78)=3.15, \mathrm{p}=0.03, \eta_{\mathrm{p}}{ }^{2}=0.11\right)$. This implies that the experiment type is affecting the pattern of d' change across the set sizes in the two groups differently. To investigate this, repeated measured ANOVA was carried out for the two groups separately. There was a significant interaction between set size and experiment in the control group $\left(\mathrm{F}(3,78)=3.31, \mathrm{p}=0.03, \eta_{\mathrm{p}}{ }^{2}=0.20\right)$ but not in the ASD group $\left(F(3,78)=1.01, p=0.398, \eta_{p}^{2}=0.07\right)$. There was also a main effect of set size in the control group $\left(\mathrm{F}(3,78)=12.66, \mathrm{p}<0.001, \eta_{\mathrm{p}}{ }^{2}=0.49\right)$ but not the ASD group $\left(F(3,78)=2.63, p=0.064, \eta_{p}^{2}=0.17\right)$. There was a main effect of experiment in both $\operatorname{ASD}\left(\mathrm{F}(1,26)=12.63, \mathrm{p}=0.004, \eta_{\mathrm{p}}{ }^{2}=0.49\right)$ and control group $(\mathrm{F}(1,26)=$ $\left.18.54, \mathrm{p}=0.001, \eta_{\mathrm{p}}^{2}=0.59\right)$.

\section{Comparison of CS error rates in experiments two and three.}

There was a significant main effect of both set size $(F(3,78)=23.29$, p <

$\left.0.001, \eta_{\mathrm{p}}{ }^{2}=0.47\right)$ and experiment $\left(\mathrm{F}(1,26)=14.44, \mathrm{p}=0.001, \eta_{\mathrm{p}}{ }^{2}=0.36\right)$. The number of errors was greater at the higher set sizes and was also higher in experiment two (blocked design). The set size by experiment interaction was significant $\left(\mathrm{F}(3,78)=3.85, \mathrm{p}=0.013, \eta_{\mathrm{p}}{ }^{2}=0.13\right)$ and reflected the greater increase in errors with increasing set size in experiment two. 


\section{Discussion}

The results of this comparison of the data from the blocked and mixed-trial experiments demonstrate some interesting findings that help elucidate the reasons for the differing patterns in the two studies. Overall, the response times were faster and the number of errors was lower in the mixed-trial experiment (experiment three). The detection rate for the CS was significantly higher for experiment three than for experiment two, but this was mainly due to an increase in the performance level of the typical group (reflected by the significant interaction between experiment and group). The ASD group appear to show more impact of perceptual load on detection rates in the mixed-trial experiment than they did in the blocked experiment (experiment two). With respect to detection sensitivity, the d' values were significantly lower in the blocked-trial experiment, and while the experiment type affected the overall level of $d$ ' in both groups, only the typical group showed a significant effect of perceptual load on detection sensitivity. The interaction between perceptual load and experiment was also significant in the control group; there was less impact of set size on the CS sensitivity in the mixed-trial experiment. As was suggested in the discussion of experiment three, it seems to be this reduction in the effect of perceptual load on detection sensitivity in the control group that eliminated the significant difference in the pattern of task performance between the two groups in the mixed-trial paradigm. Macdonald and Lavie (2008) also found that mixing the trials resulted in a less pronounced effect of perceptual load on CS sensitivity and suggest that this may be due to carry over effects from one level of load to another thus altering the load of the subsequent trial. Alternatively, it may reflect a strategy difference between the two paradigms. Theeuwes et al. (Theeuwes et al., 2004) suggested that when a blocked design is employed, the search strategy is set 
differently for each of the different blocks. If strategy differences were involved, however, it would be predicted that this would offer an advantage in the blocked design. Using expectancies and setting a search strategy for each block is done precisely because it allows the task to be performed more effectively. When the trials are mixed, therefore, one would expect to see an increase in the RTs and the error rates. The data from the comparison analyses, however, indicates that this is not the case. In all cases, the error rates were lower and the RTs were shorter in the mixedtrial experiment. This is incompatible with the hypothesis that removing the influence of strategy by mixing the trials resulted in the elimination of the performance differences seen between the two groups.

After inspecting the data, what seems to be a more plausible explanation is that practice effects are responsible for the discrepancy between the two experiments. Unlike previous studies such as those by Macdonald and Lavie (2008), the same participants performed both tasks - and always performed the blocked experiment first. In retrospect, this was not advisable and seems to have resulted in a vast improvement on the second experiment. While practice effects appear to have caused an increase in the performance level of both groups on the mixed design, the ASD group were already performing at a very high level in experiment two. This may have led to a ceiling effect and meant that any possible increase would be on a smaller scale for this group. As a result, the rise in performance level has caused the control group performance to become closer to the ASD performance and significant differences between the behaviours are eliminated.

The comparison ANOVA results back up this hypothesis. The detection rate and d' comparisons both show that the control group is demonstrating a much bigger rise in performance level in the mixed design. The results also show, although no 
longer significant, the ASD group is still showing less influence of perceptual load on detection sensitivity than the control group.

Given this hypothesis, it may not be appropriate to take the results of experiment two as an accurate reflection of task performance. It is necessary to repeat the mixed-trial design with a new set of participants in order to draw conclusions regarding any potential strategy influences.

\section{Overall Discussion}

Having concluded that data from the mixed-trial experiment may have been affected by practice or carry over effects, the conclusions drawn from this study regarding conscious perception in ASD will be based primarily on the blocked-trial experiment (experiment two).

The results presented here suggest that while the control group's conscious perception of the CS was modulated by perceptual load, the ASD group show consistently high detection sensitivity across all the set sizes. This resulted in the ASD individuals detecting the CS significantly more than the control group at the higher set sizes. This observation is consistent with the findings of experiment one where the ASD group showed increased distractibility at high levels of perceptual load. It appears, therefore, that the increased perceptual capacity in ASD - as determined by the indirect RT comparison methods - is also reflected in conscious perception.

While this increased sensitivity at high set sizes was in line with the original hypothesis, the demonstration that the ASD group did not show a significant effect of perceptual load on the detection sensitivity was more surprising. It had been predicted that both groups would show a drop in d' levels as the perceptual load of 
the central search task was increased, with the ASD group showing the drop in performance at a higher set size than the control group. There are a number of reasons why this trend may not have been seen.

Firstly, it may be the case that perceptual load theory does not apply to conscious perception in ASD. The data presented in chapter three showed a modulation of selective attention by perceptual load and hence it seems unlikely that this pattern is no longer seen in conscious perception. Furthermore, the d' data shows that, although the trend is not significant, there is a slight drop in detection sensitivity for the ASD group as the set size increases.

A more likely explanation is that the increased perceptual capacity means that even at set size six (the highest set size used in this study) individuals with ASD have not exhausted their processing capacity and therefore can continue to effectively detect the CS. This is consistent with the fact that the error rates were no higher in the ASD group - and therefore individuals were still performing the task successfully. It may well be the case that the central task used in the current study did not reach a level of perceptual load that would result in the necessity for individuals with ASD to exhibit early selection (and hence fail to detect the CS) in order to efficiently perform the letter-search task. It is possible that if the set size was increased further, a more significant decline in CS detection would be observed for the ASD group. It is important to now carry out a further experiment with larger set sizes in order to investigate whether an effect of perceptual load on conscious perception can be observed in the ASD group.

As with the results presented from experiment one, the findings from this experiment do not demonstrate enhanced visual search performance. There was no overall difference between the groups in the response times for the central task. 
Again, this may be due to ceiling effects at the low set sizes and an impact of CS processing by the individuals with ASD at the higher set sizes.

The preliminary results presented here, however, offer the first suggestion that increased perceptual capacity in ASD is reflected by the increased ability to detect a critical stimulus at high levels of perceptual load. 


\section{Chapter 5: Visual Short Term Memory Capacity in ASD}

\section{Introduction}

The data presented in the previous experimental chapters suggest that individuals with ASD have an increased perceptual capacity relative to control individuals. This conclusion was drawn from the observation that at high levels of perceptual load, individuals with ASD continue to be distracted by irrelevant distractor stimuli in a selective attention task (chapter three) and continue to detect the critical stimulus (CS) in a dual-task paradigm (chapter four). Load theory states that if a central task does not exhaust perceptual capacity then elements from the surrounding environment will also be processed; the capacity is filled automatically. This reasoning, together with results from a selective attention task under various levels of load, has already been used to glean information regarding the perceptual capacity of subgroups within the typical population. Young children and the elderly have been shown to have reduced capacity compared to young adults (HuangPollock et al., 2002; Maylor \& Lavie, 1998). The pattern of increased distractibility and CS detection under high perceptual load seen for the ASD group in experiments one and two (given that there was no drop in central-task performance) suggests that it took a greater level of load to exhaust the perceptual capacity of the individuals with ASD.

One hypothesis arising from experiments one and two is therefore that individuals with ASD have increased perceptual capacity. The aim of this next experiment was to test this hypothesis by directly measuring a cognitive element that reflects perceptual capacity. Visual short-term memory was chosen as an appropriate 
measure as it provides a value for the amount of visual information that can be encoded at any one time.

\section{Visual Short-Term Memory}

The term visual short-term memory (VSTM) refers to the temporary storage of visual information over an extended period of time and is different from both iconic and long-term memory. Phillips (1974) demonstrated the existence of two distinct classes of visual memory. He observed a process of high capacity sensory storage (iconic memory) which was very brief (100ms storage time), could be disrupted by a stimulus mask and was tied to spatial position. Conversely, the 'shortterm visual memory' he highlighted had a limited capacity, showed a duration of 600ms (followed by a slow degradation over at least nine seconds), was not undermined by masking and was not tied to spatial position.

Visual short-term memory is most commonly assessed using changedetection paradigms. In these experiments, participants are presented with two arrays which are separated by a short temporal interval, and asked to indicate whether the arrays are the same or different. One such procedure that has been widely used by many research teams since its conception is the task devised by Luck and Vogel (1997). Participants were briefly shown an array of coloured squares which varied in size between one and twelve squares. Following a blank interval, the array was shown again. In half the trials, one of the squares had changed colour whereas in the other trials the sample and test array were identical. Participants were required to indicate whether a change had occurred. Results indicated that task performance was at ceiling for arrays of up to two squares, dropped slightly for arrays of three to four 
squares, and fell sharply as the array size was increased further. One would predict that, once the number of squares in the array exceeded short-term memory capacity, the participant would begin to make errors on the change detection task. They therefore concluded, in line with many other studies, that typical adults have the visual capacity to encode approximately four items (Kahneman et al., 1992; Luck \& Vogel, 1997; Sperling, 1960). By modifying the procedure, Luck \& Vogel (1997) and Vogel, Woodman \& Luck (2001) also demonstrated that visual memory stores integrated objects rather than individual features, and that verbal load does not affect task performance. They showed that participants were able to retain both the colour and the orientation of three to four objects, and that objects defined by a conjunction of four features were held in visual working memory just as well as single-feature objects. The capacity of visual working memory appears to increase with development, reaching adults levels by 10 years of age (Riggs et al., 2006).

At this point, it is important to mention terminology. The terms 'short-term memory' and 'working memory' are often used interchangeably, and the distinction between them is frequently unclear due to different researchers using different definitions. What appears to be the case is that they are related concepts with different historical origins (Cowan, 2008). Atkinson and Shiffrin (1968) used 'short term memory' to reflect the cognitive components that can temporarily hold a limited amount of information in an accessible state. Working memory became a more dominant term within the field after Baddeley and Hitch (1974) put forward a multicomponent model for temporary memory. The term 'short-term memory' was used to refer to the single store of information (as in the Modal Model of memory associated with (Atkinson \& Shiffrin, 1971) while 'working memory' was used to refer to the entire multi-component system and processes that hold and manipulate 
information in short-term memory. More recently, Cowan and colleagues (Conway et al., 2002; Cowan, 1995) have aimed to clarify the confusion by asserting that short-term memory is a simple storage buffer whereas working memory consists of both a storage component and an attention component. Working memory is used to maintain memory representations when distraction, attention shifts or concurrent processing threatens to undermine them (Conway et al., 2002). Cowan also asserts, however, that no one task is a 'pure' measure of short-term or working memory. The more that a task requires a participant to perform effortful and controlled processing, the more that task will reflect working memory capacity rather than short-term memory capacity (which would be invoked in a task that required more automatized skills).

Both terms have been used with respect to varieties of the change-detection paradigm (outlined above) that are most commonly used to obtain capacity estimates. While some studies have used the task to investigate visual short-term memory (Todd \& Marois, 2004), others consider that the results of such a task reflect visual working memory (Luck \& Vogel, 1997; Riggs et al., 2006; Rouder et al., 2008). The task used within this thesis aims to provide a measure of visual short-term memory. Participants were asked to perform a change detection task that did not involve any distractors, concurrent processing or loading of working memory. An array of coloured squares (two, five, eight or twelve) was presented briefly, followed by a blank delay, a mask and lastly a single test square. Participants were asked to indicate whether the test square was the same colour as the one that had been in that location within the initial array. Examining the relative performance across the various array sizes can give an indication of how many squares can be processed at 
any one time. We consider this measure analogous to the perceptual capacity that we referred to in experiments one and two.

\section{Calculating visual short-term memory capacity}

While it is possible to obtain a rough estimate for short-term memory capacity by observing the array size at which task performance drops (as explained above), it is also possible to calculate a more precise capacity value using Pashler's probabilistic model for change detection (Pashler, 1988). Pashler reasoned that if an individual has a visual capacity of $k$ items, then performance should be at ceiling for arrays of $n$ squares, where $n \leq k$. When the array size exceeds $k$, the participant can still only encode the same number of squares and therefore the chance that the participant encoded any one particular square and can correctly detect a subsequent change is $k / n$. If the relevant square in the sample array was not encoded then the participant must guess, resulting in either a 'miss' (a change trial where the individuals responds 'no change') or a 'false alarm' (a no change trial where the individual reports a change). Pashler devised a formula that uses the hit rate (proportion of changes correctly detected) and the false alarm rate at any given array size to provide a value for visual short-term memory capacity:

$$
k=(\mathbf{n}(\mathbf{h}-\mathrm{g})) \div(\mathbf{1}-\mathrm{g})
$$

where $\mathrm{k}$ is visual short-term memory, $\mathrm{n}$ is array size, $\mathrm{h}$ is the hit rate and $\mathrm{g}$ is the false alarm rate.

While this equation provides an estimate for capacity, it does not take into account the accuracy of responses in the no-change situations. This may become problematic in situations where an individual has a strong bias towards responding 
'change detected' and therefore achieves a high hit rate that is due to indiscriminate guessing rather than a true reflection of task performance. Cowan (2001) has put forward a revised version of the formula that also incorporates the proportion of 'correct rejections' (accurately responding 'no change' on no change trials). The formula now states that:

$$
k=n(h+c r-1)) \div c r
$$

where $k$ is the visual short-term memory capacity, $n$ is array size, $\mathrm{h}$ is the hit rate and $c r$ is the proportion of correct rejections. Cowan's revised equation that will be used within this study.

\section{Predictions}

It was predicted that individuals with ASD would show superior performance on the short-term memory task when compared to control participants. If, as suggested by the previous experiments within this thesis, the young adults with ASD have a higher perceptual capacity and can take in more visual information at any one time, then one would expect that such individuals would be able to encode more of the squares in the sample array. This would result in more accurate responses to the test square and, consequently, a higher calculated value for visual short-term memory capacity. This hypothesis fits well with existing work on change detection in ASD. It has been shown that individuals with ASD show an increased ability on spot-thedifference tasks (Teunisse et al., 2001). Similarly, in a recent study, Smith and Milne (2009) showed that the participants with autism were less prone to change blindness. Change blindness is the phenomenon whereby unexpected changes in the visual field are overlooked - usually as a result of distraction such as camera angle change in films, luminance change or a visual saccade. When shown videos with continuity 
errors, the adolescents with autism were significantly better at detecting the errors. A study by Fletcher-Watson et al. (Fletcher-Watson et al., 2006), however, failed to show a difference between the ability of control individuals and individuals with ASD to detect changes in visual scenes. Likewise, Burack et al. (2009a) also reported no difference in performance on a change detection task. However, when task performance was related to the developmental level of the participants a group difference emerged. In the typically developing group, the number of errors on the task decreased as the developmental level of the children increased (as measured by a non-verbal, visuo-spatial test of intelligence). Conversely, there was no relationship between task performance and developmental level in the ASD group. Given that the visual short-term memory paradigm used within this thesis involves detecting when the colour of the test square has changed, these previous studies on change perception may add weight to the prediction regarding enhanced visual short-term memory capacity.

\section{Participants}

Participants in this study were 16 adults with Asperger's Syndrome and 16 typical adults. The participants were diagnosed and recruited in the same manner as in the previous experiments and were matched for chronological age and non-verbal IQ using the matrix reasoning sub-scale from the Wechsler Abbreviated Scale for Intelligence (WASI) (Wechsler, 1999) (see table 5.1). 
Table 5.1 Descriptive Statistics for each Group

\begin{tabular}{llcccc}
\hline Group & Statistic & $\begin{array}{c}\text { Age } \\
\text { (years: } \\
\text { months) }\end{array}$ & $\begin{array}{c}\text { WASI } \\
\text { vocabulary } \\
\text { subtest }\end{array}$ & $\begin{array}{c}\text { WASI } \\
\text { matrix } \\
\text { reasoning }\end{array}$ & $\begin{array}{c}\text { WASI } \\
\text { full scale IQ } \\
\text { (2 subtests) }\end{array}$ \\
\hline ASD $(\mathrm{n}=16)$ & mean & $24: 9$ & 64.6 & 54.5 & 117 \\
12 males & S.D & $4: 7$ & 5.7 & 10.2 & 13.5 \\
4 females & range & $18: 09-33: 4$ & $58-72$ & $37-67$ & $95-136$ \\
\hline Control $(\mathrm{n}=16)$ & mean & $26: 9$ & 66.9 & 54.1 & 119 \\
11 males & S.D. & $3: 4$ & 6.0 & 8.3 & 11.4 \\
5 females & range & $21: 5-32: 8$ & $52-74$ & $41-66$ & $101-137$ \\
\hline
\end{tabular}

Independent samples t-tests indicated that there was no difference between the groups on any of the above measures (all $\mathrm{p}$ values $>0.1$ )

\section{Methodology}

\section{Apparatus and Stimuli}

Visual basic was used to create computer based stimuli that were presented on a custom built small form desktop computer and displayed on a ProLite 15" flat LCD screen (1280 x 1024 pixel resolution, 2ms response rate). Viewing distance was $60 \mathrm{~cm}$.

The stimulus arrays were composed of two, five, eight or twelve coloured squares that were presented on a grey background. Each square measured $0.75^{\circ}$ by $0.75^{\circ}$ visual angles and was randomly positioned within a $9.8^{\circ}$ by $7.3^{\circ}$ region of the screen, with no less than $2^{\circ}$ between the centre of each square. No squares were presented within the central $2^{\circ}$ of the screen. The colour of each square was assigned randomly, with replacement, from a choice of black, white, red, light blue, light green, yellow, orange, cyan, purple, dark blue and dark green. 


\section{Procedure}

Participants were told that they would briefly see a set of coloured squares on the centre of the screen. They were informed that these squares would then disappear and be replaced by one square that was in the same location as one from the initial array but which may, or may not, be the same colour as the square that had originally been in that position. They were instructed to indicate, with a key-press, whether the colour of the single square had changed or whether it was the same as that of the corresponding square from the sample array.

Each trial began with the presentation of a fixation cross for $1000 \mathrm{~ms}$, followed by the sample array which remained on the screen for 500ms. This was replaced by a blank screen $(500 \mathrm{~ms})$ and then a mask (same as sample array but with each square filled with a multicoloured checker board pattern) for 500ms and then the test probe: a single square in the same position as one from the sample array with the colour either changed or unchanged (50\% of trials with each condition). Participants were then given three seconds to indicate whether or not the colour had been changed and the response time was recorded. If no response was given within the time limit then the trial was classified as a 'time-out' error. 72 trials of each array size were created and these were randomly mixed and presented in eight blocks of 36 trials - with participants able to take breaks between blocks if required (see figure 5.1).

Prior to performing the computer based task, participants were shown a white piece of paper on which a sample array of five squares had been drawn. They were asked to name the colours (to ensure normal colour vision) and were then asked to memorise the colour and location of the squares. Participants were then shown a second piece of paper on which one unchanged square from the first array was drawn 
and asked to confirm that the colour of this square was the same as in initial array. They were then shown one further example where a colour-change had occurred. Following successful completion of the paper examples, a set of computer based practice trials were performed where participants were given feedback regarding their accuracy. The experimental trials were then run. There was no accuracy feedback on the main experimental task.

Figure 5.1 Time-course of experimental trials

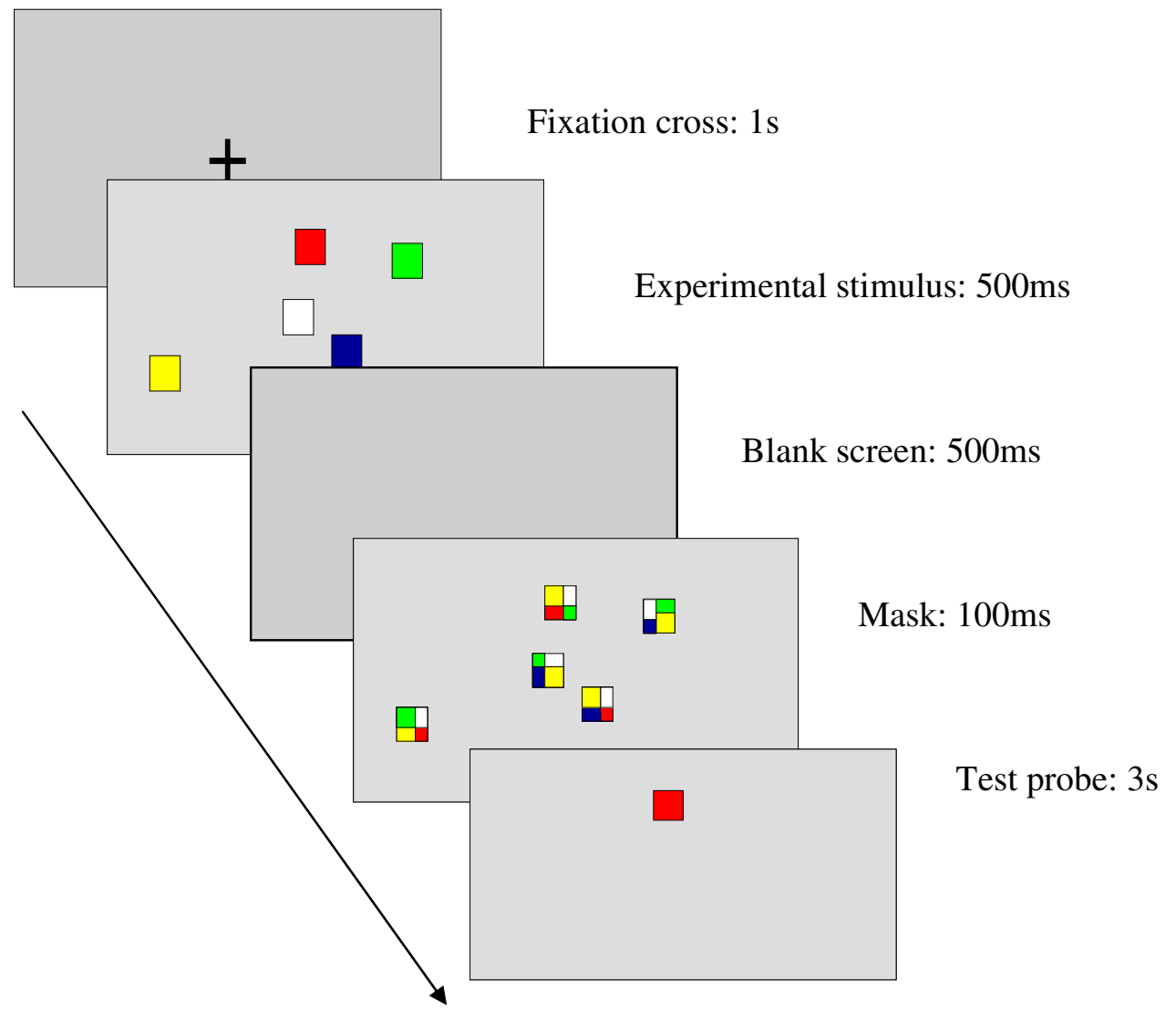




\section{Results}

The number of correct responses on 'change trials' (hits), and 'no change' trials (correct rejections), were calculated for each participant at each array size. These scores were then added together to provide an overall task performance score (see tables 5.2 and 5.3 for mean values). The number of false alarms (reported a change when no change occurred) was also obtained for each participant at each array size. This provided an indication of guessing rate. An ANOVA was then performed on the hit rate, the total correct response rate and the false alarm rate with array size (two, five, eight $\&$ twelve) as the within-subject factor and group (ASD vs. control) as the between subject factor.

\section{Percentage change detection}

Table 5.2 Mean $\%$ of changes detected on change-trials (hit rate) and standard deviation for each group at each array size

\section{Mean hit rate (Standard Deviation)}

\begin{tabular}{lllll} 
Array size & \multicolumn{2}{c}{ ASD } & \multicolumn{2}{c}{ Control } \\
\hline $\mathbf{2}$ & 89.2 & $(0.68)$ & 88.5 & $(6.56)$ \\
$\mathbf{5}$ & 79.9 & $(0.93)$ & 79.0 & $(1.33)$ \\
$\mathbf{8}$ & 77.8 & $(1.33)$ & 72.4 & $(1.37)$ \\
$\mathbf{1 2}$ & 76.0 & $(1.30)$ & 73.1 & $(1.16)$ \\
\hline
\end{tabular}


Figure 5.2 Graph of mean percentage of changes detected.

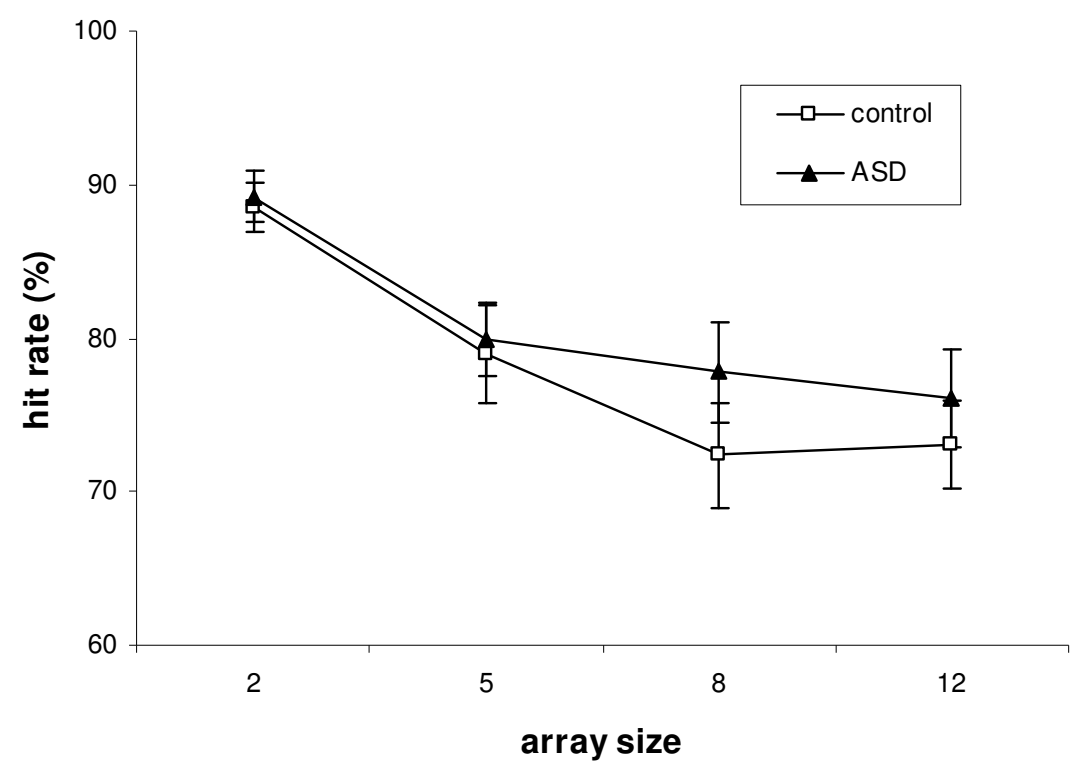

Analysis of the hit rates at each array size revealed a main effect of set size $\left(\mathrm{F}(3,90)=28.56, \mathrm{p}<0.001, \eta_{\mathrm{p}}^{2}=0.45\right)$. Inspection of the means indicated that this is a reflection of the increasing number of errors as the array size was increased (see table 5.2). There was no main effect of group $\left(\mathrm{F}(1,30)=0.08, \mathrm{p}=0.784, \eta_{\mathrm{p}}{ }^{2}<0.01\right)-$ neither group made significantly more errors - and no significant interaction between group and array size $\left(\mathrm{F}(3,90)=0.87, \mathrm{p}=0.459, \eta_{\mathrm{p}}^{2}=0.03\right)$. 


\section{Overall correct response rate}

Table 5.3 Mean \% correct responses and standard deviation for each group at each array size.

\section{Mean correct response rate (Standard Deviation)}

\begin{tabular}{lllll} 
Array size & \multicolumn{2}{c}{ ASD } & \multicolumn{2}{l}{ Control } \\
\hline $\mathbf{2}$ & 87.6 & $(9.34)$ & 91.2 & $(4.60)$ \\
$\mathbf{5}$ & 70.1 & $(12.2)$ & 73.3 & $(9.23)$ \\
$\mathbf{8}$ & 62.5 & $(12.6)$ & 66.3 & $(8.22)$ \\
$\mathbf{1 2}$ & 57.9 & $(5.14)$ & 58.9 & $(8.87)$ \\
\hline
\end{tabular}

Figure 5.3 Graph of mean correct responses.

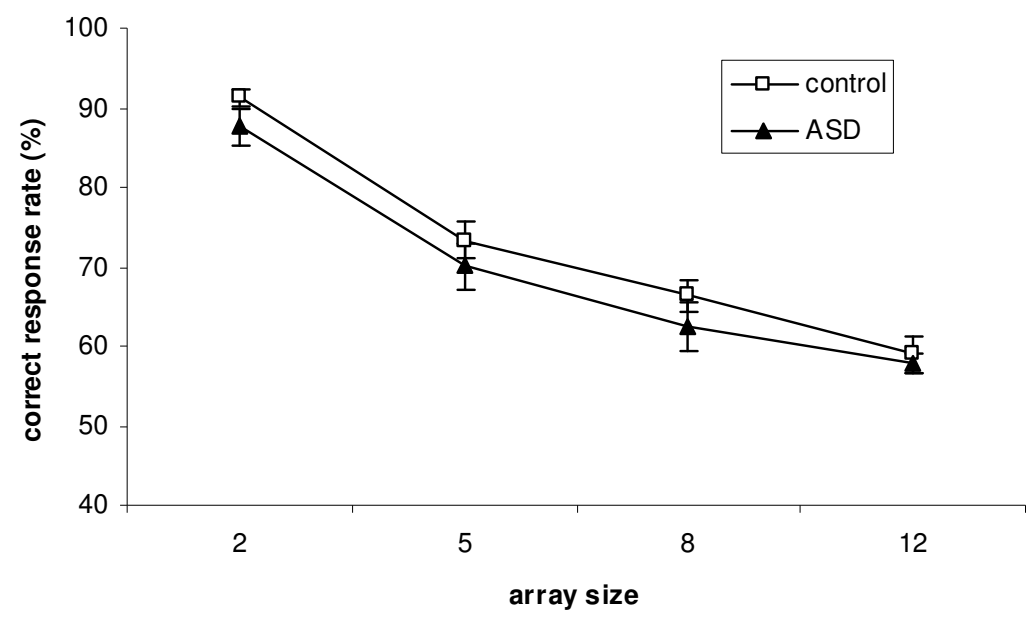


Analysis of the correct response rate for each participant at each array size revealed a main effect of set size $\left(F(3,90)=162.00, p<0.001, \eta_{p}^{2}=0.84\right)$. As with the hit rates, this is a reflection of the increasing number of errors made at the larger array sizes (see table 5.3). There was no main effect of group $(\mathrm{F}(1,30)=1.15$, $\left.\mathrm{p}=0.2914, \eta_{\mathrm{p}}{ }^{2}=0.04\right)$ and no significant interaction between group and array size $\left(\mathrm{F}(3,90)=0.37, \mathrm{p}=0.774, \eta_{\mathrm{p}}^{2}=0.01\right)$.

False Alarm Rate

Table 5.4 Mean \% false alarm rate and standard deviation for each group at each array size.

Mean false alarm rate (Standard Deviation)

\begin{tabular}{lllll} 
Array size & \multicolumn{2}{c}{ ASD } & \multicolumn{2}{c}{ Control } \\
\hline $\mathbf{2}$ & 13.5 & $(16.2)$ & 5.24 & $(3.00)$ \\
$\mathbf{5}$ & 38.7 & $(19.2)$ & 29.1 & $(13.3)$ \\
$\mathbf{8}$ & 51.6 & $(18.7)$ & 39.5 & $(12.1)$ \\
$\mathbf{1 2}$ & 59.2 & $(10.4)$ & 53.4 & $(11.8)$ \\
\hline
\end{tabular}


Figure 5.4 Graph of mean false alarm rate.

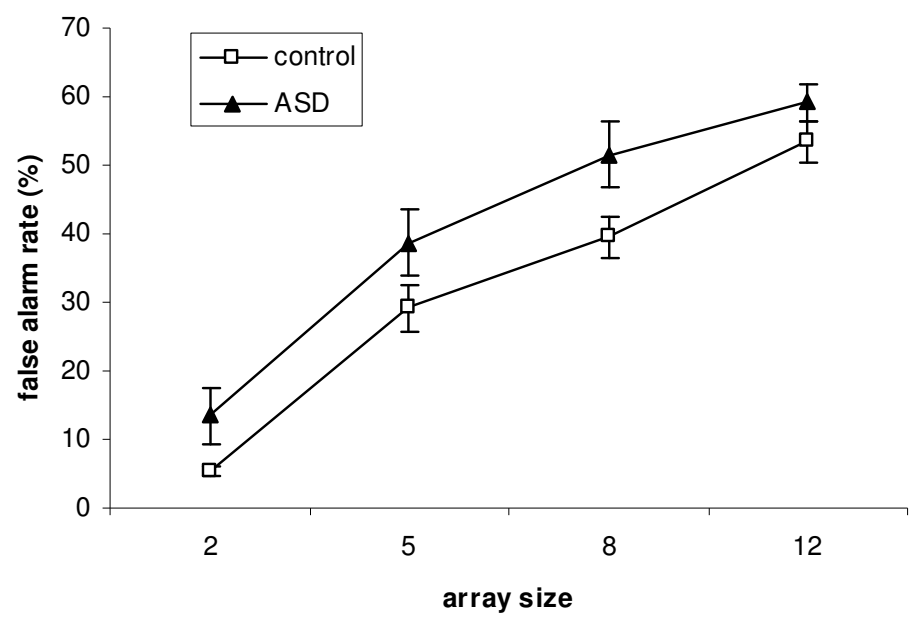

Analysis of the false alarm rate for each participant at each array size revealed a main effect of set size $\left(\mathrm{F}(3,90)=144.36, \mathrm{p}<0.001, \eta_{\mathrm{p}}{ }^{2}=0.83\right)$. Participants seemed to be guessing more as the array size increased (see table 5.4). There was also a main effect of group $\left(\mathrm{F}(1,30)=5.00, \mathrm{p}=0.033, \eta_{\mathrm{p}}{ }^{2}=0.15\right)$. Inspection of the means indicated that this was a reflection of slightly higher guessing rates across all array sizes within the ASD group. The interaction between group and array size was not significant $\left(F(3,90)=0.62, \mathrm{p}=0.605, \eta_{\mathrm{p}}{ }^{2}=0.02\right)$.

\section{Response Bias}

Calculating response bias provides an indication of whether participants have an underlying bias towards responding 'change' or 'no change'. In the present experiment, the colour of the test square was changed in $50 \%$ of the trials and remained the same in the other $50 \%$. Therefore, the tendency to respond 'change' and 'no change' should be equal. Task instructions or demands can, however, affect this equilibrium. By exploring response bias, one can elucidate whether there are 
response criteria that may be influencing the results. Response bias was calculated for each participant at each array size using the formula $\quad \beta=\left\{\frac{Z_{S \mid n}^{2}-Z_{S \mid s}^{2}}{2}\right\}$ (see p.90, chapter four). A value greater than one indicates that a participant has a tendency to respond 'change', whereas a value below one reflects a bias towards responding 'no change'.

Table 5.5 Mean response bias $(\beta)$ and standard deviation for each group at each array size.

Mean response bias (Standard Deviation)

\begin{tabular}{llllll} 
Array size & \multicolumn{2}{c}{ ASD } & \multicolumn{2}{c}{ Control } \\
\hline $\mathbf{2}$ & 1.20 & $(0.94)$ & 0.75 & $(0.60)$ \\
$\mathbf{5}$ & 1.43 & $(0.69)$ & 1.46 & $(0.65)$ \\
$\mathbf{8}$ & 1.86 & $(1.86)$ & 1.27 & $(0.46)$ \\
$\mathbf{1 2}$ & 1.73 & $(1.16)$ & 1.27 & $(0.35)$
\end{tabular}

Analysis of the response bias values indicated that the main effect of set size was not significant $\left(F(3,90)=2.71, p=0.077, \eta_{p}^{2}=0.08\right)$. This is, however, close to significance and appears to reflect a tendency to respond 'change' slightly more often at the higher array sizes. There was no significant main effect of group $(\mathrm{F}(1$, 30) $\left.=3.88, \mathrm{p}=0.058, \eta_{\mathrm{p}}{ }^{2}=0.11\right)$ but again the difference is close to significance. The ASD group seems a little more likely to respond 'change'. There was no significant interaction between group and array size $\left(\mathrm{F}(3,90)=0.72, \mathrm{p}=0.542, \eta_{\mathrm{p}}{ }^{2}=0.02\right)$. 


\section{Capacity}

Cowan's revised formula (see p.116) was used to calculate capacity estimates for each participant at each array size. The mean capacity calculations for each group are displayed in table 5.6 and figure 5.5.

Table 5.6 Mean visual short-term memory capacity and standard deviation for each group at each array size.

\section{Mean capacity (Standard Deviation)}

Array size

ASD

Control

2

$1.50 \quad(0.37)$

$1.72(0.19)$

5

$2.01 \quad(1.22)$

$2.36 \quad(1.95)$

8

$2.00 \quad(2.01)$

$2.81 \quad(1.39)$

12

$1.90 \quad(1.23)$

$2.36 \quad(1.95)$

Figure 5.5 Graph of mean values for visual short-term memory capacity.

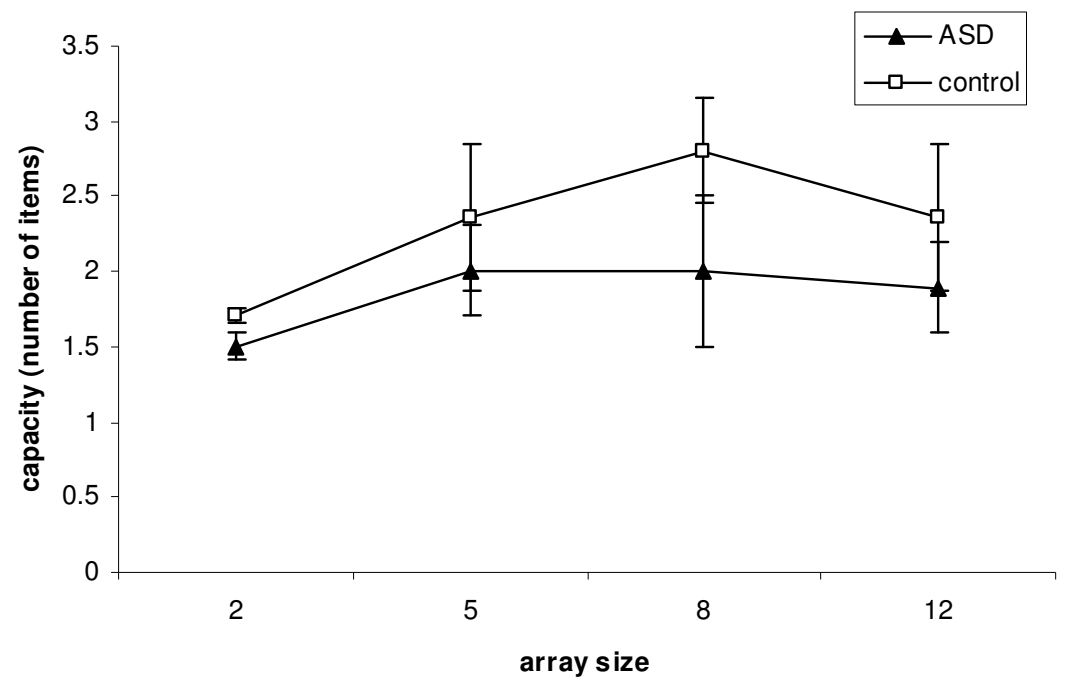


Using ANOVA to analyse the capacity estimates highlighted a main effect of set size $\left(\mathrm{F}(3,90)=3.81, \mathrm{p}=0.013, \eta_{\mathrm{p}}{ }^{2}=0.11\right)$. This is due to the fact that the capacity value rose as the array size was increased. Once the number of items in the array exceeds capacity then the value will not increase any further and this plateau indicates the true visual short-term memory capacity. There was no main effect of group $(F(1,30)$ $\left.=1.9, \mathrm{p}=0.179, \eta_{\mathrm{p}}{ }^{2}=0.06\right)$ and no significant interaction between group and array size $\left(F(3,90)=0.52, \mathrm{p}=0.669, \eta_{\mathrm{p}}^{2}=0.02\right)$.

In order to compare the overall capacities of the two groups, it is necessary to obtain one value for each participant. According to Cowan, the formula should yield a constant result once the array size exceeds a participant's capacity. By examining the graphs displaying percentage hits and overall correct responses, and noting the reduction in task performance as the array size increased, it could be deduced that an array of eight squares was greater than the visual short term memory capacity for both participant groups. Therefore, the value calculated for each participant at this array size was used as the overall capacity estimate (ASD capacity $=2.0$, control capacity $=2.8$, see table 5.6). An independent samples t-test indicated that there was no significant difference between the capacity estimates of two groups $(t(30)=-1.3$, $\mathrm{p}=0.197)$. 


\section{Discussion}

This experiment investigated, for the first time, the extent of visual short-term memory capacity in adults with ASD. In the results presented here, no evidence was found of any difference between the capacity of individuals with ASD and IQmatched controls. This is similar to the findings of Fletcher-Watson et al. (2006) who demonstrated that the ability to detect changes was equivalent in both ASD and control groups, and may also be consistent with Burack's work (Burack et al., 2009a) that suggests differences will only be seen when developmental level is taken into account. The absence of any increased level of visual short-term memory capacity would suggest that the increased distraction at high perceptual load seen in ASD in experiments one and two is not due to increased capacity.

An alternative suggestion is that the increased distractibility was due to increased working memory load in the ASD group. Lavie and colleagues have extended load theory to include the impact of working memory load and cognitive control on selective attention. They suggest that in order to direct attention appropriately, individuals must maintain stimulus priorities in working memory - i.e. to remember which stimuli are relevant. Therefore, loading working memory processes should result in reduced differentiation between high- and low-priority stimuli (targets and distractors) and lead to the opposite effect from that of perceptual load; as working memory load increases, the extent of distractor processing will also increase (de Fockert et al., 2001; Lavie et al., 2004c). Given the absence of an increased in VSTM capacity, it could therefore be suggested that the increased effect of distractors seen in experiments one and two was due to task demands disproportionately loading the working memory processes of the individuals with ASD. Indeed it has been suggested that working memory is one of the components of 
the executive system that are impaired in ASD (Bennetto et al., 1996; Russell et al., 1996). This will be discussed more fully in chapter seven.

However, we need to be cautious before ruling out the perceptual capacity explanation on the basis of the result regarding VSTM. The VSTM task used within this thesis, and change detection paradigms in general, are extremely sensitive to small manipulations in procedure. For example, the conflict between the results found by Smith and Milne (2009) that those of Fletcher-Watson et al. (2006) and Burack et al. (2009a) may be due to procedural differences. The paradigms used by both Fletcher-Watson and Burack involved the presentation of picture pairs and participants were asked to identify what alteration had been made. It was therefore known that a change must be found on each trial. On such tasks, no difference was found between ASD and control groups. Conversely, Smith and Milne used film clips, only some of which contained continuity errors. Participants were informed that some of the clips may contain a mistake and, following stimulus presentation, were then asked if they had noticed anything unusual. On this task, the individuals with ASD performed better than control individuals; spotting more of the continuity errors.

Bearing in mind the impact of relatively small task manipulations in these previous studies, there are a number of possible explanations for my null result found on experiment four. Firstly, it may be due to differing response criteria in the individuals with ASD. The difference in response bias between the two groups was close to significance, with the ASD group showing more of a tendency to respond 'change' and the ASD group made a significantly higher number of false alarm errors (responding 'change' in 'no change' conditions). This is accounted for in Cowan's equation, and leads to a reduction in the capacity estimate. This altered bias 
may have arisen from the way in which a single probe square, rather than the whole array, was used to test VSTM within the task. The previous studies are split with regard to this aspect. Some present the whole array at the test phase (Luck \& Vogel, 1997; Riggs et al., 2006) while others present only the relevant square (Rouder et al., 2008; Todd \& Marois, 2004). The latter was selected for the task presented within this thesis as it ensures that the amount of decision making is kept constant throughout the trial types. If the whole array is presented as the probe, then participants must consider each square before being able to indicate whether any colour change has occurred. This means that the amount of decision making increases with array size and the difficulty of the task increases exponentially. Using a single-probe test also removes any 'grouping' advantage that might arise in the higher set sizes. It is my belief, however, that in selecting the single probe test method, the nature of the task is radically changed and this has implications for its use with a clinical population such as ASD. This method relies on being able to understand that, although the single square is in a different context and presentation style, it corresponds to one that was presented in the sample array. The individuals with ASD may have been overly literal and responded 'change' because there was only one square in the test phase- rather than several squares as in the sample array.

Secondly, the difference between the higher array sizes may have been too great. Due to the prediction regarding increased VSTM capacity in ASD, it was important to include trials with a large array. A failure to do this may have resulted in the ASD group performing at ceiling and therefore a true capacity value would be impossible to determine. The maximum array size was therefore set at 12 squares. It is also important to maximise the number of trials of each array size (in order to increase statistical power and negate the effect of sample variance as far as possible) but if the 
overall number if trials is too great then fatigue can ensue. For this reason, the number of different array sizes included in the experiment was kept to a minimum (two, five, eight and twelve). In hindsight, however, it seems that an array size of eight is sufficient to exceed the capacity of both groups. It may also be the case that differences between the groups may have been evident at the point at which the capacity limits are just being reached. For this reason, it would be important to repeat the experiment using a more fine-tuned set of array sizes such as two, three, four and six.

Lastly, a recent study has presented data concerning abnormal colour perception in ASD. Franklin and colleagues (Franklin et al., 2008) compared children with ASD and typically developing children on visual search and matchingto-sample and discrimination tasks that involved colour perception. In the visual search task, participants were shown a grid of coloured squares and asked to identify the odd one out, while in the delayed matching-to-sample task the participants were shown the target design and then subsequently asked to identify the initial stimulus from a pair of possible stimuli. The results demonstrated that the children in the clinical group were less accurate at colour memory, search and chromatic targetdetection than the controls. They conclude that the 'enhanced perceptual functioning' that has been observed in ASD does not extend to the domain of colour. This finding was unexpected as the few existing studies in this area suggest that individuals with ASD process colour more readily and that colour cues can be detected more easily (Brian et al., 2003; Kovattana \& Kraemer, 1974). Franklin's findings however, have ramifications for the data presented within this chapter. A central requirement of the visual short-term memory task employed here was to memorise and discriminate between different colours and therefore indicate whether the colour of the test square 
was the same as the corresponding square in the sample array. If processing colour is problematic for individuals with ASD then the task would be rendered disproportionately difficult, and any perceptual advantage derived from enhanced capacity would be undermined. The absence of any significant difference between the groups fits well with this idea that, for the individuals with ASD, increased ability and deficit are cancelling each other out to result in performance which matches that of typical adults. If a true measure of visual short-term memory capacity is to be obtained for individuals with ASD, a task that does not involve colour perception must be used. Such a task could involve encoding a sample array of different shapes or squares with different fill patterns.

In order to address the various drawbacks and concerns with the existing methodology before concluding that perceptual capacity is not enhanced in ASD, a modified version of the experiment should be performed. Rather than using coloured squares, a set of lines - defined by orientation - would overcome any issue of abnormal colour processing. Array sizes of two, three, four and six would also allow more detailed information regarding the capacity of each group to be ascertained.

In conclusion, the findings from the thesis to this point suggest that perceptual capacity may be enhanced in ASD. This increased level is seen both in unconscious and conscious tasks of attention, and fits well with anecdotal and experimental reports of superior performance in certain areas within the condition. In the second part of this thesis, the research will focus on the question of selective attention and perceptual load in the social domain. Given that social processing is a fundamental impairment in ASD, this is a vitally important area of investigation. 


\section{Chapter 6: Selective Attention and Perceptual Load with}

\section{Face Distractors}

\section{Introduction}

The research within this thesis can be split into two parts: the first dealing with socially-meaningless stimuli, the second with social attention - in particular attention to faces. Face perception is a crucial process that enables us to interact with those around us (Ellis \& Young, 1988). Processing the faces of others involves recognition, an evaluation of the degree of familiarity, the gender, the emotional state, lip-reading and extraction of a great deal of other socially valuable information. The essential nature of successful face perception and processing is highlighted by the debilitating consequences when this process is impaired. As discussed earlier in this thesis, one of the fundamental deficits with ASD is a problem with social behaviours. Individuals seem to fail to use information from the face such as eye gaze and expression, and therefore show inappropriate, if any, social interaction (see Spezio et al., 2007). The reason for these altered behaviours has been extensively debated within the literature but no consensus has yet been reached. Existing findings point to a dysfunction of face processing regions in the brain (Baron-Cohen et al., 2000; Dawson et al., 2005) and/or a lack of interest in social stimuli in early childhood (Dawson et al., 2005; Grelotti et al., 2002; Schultz, 2005). The latter is clearly intrinsically linked to attention - with individuals with ASD assigning less attentional resources to social stimuli than typical controls (Klin et al., 2002; Swettenham et al., 1998). Anecdotally, this is well documented by parents who 
report that children with ASD pay a great deal of attention to certain toys or objects but not to family members or contemporaries.

With this in mind, it is important to extend the work on selective attention and perceptual load to include social stimuli. In an interesting manipulation of the perceptual load procedure, Lavie, Ro and Russell (2003) carried out a further experiment using famous faces as the distractors to explore whether, with such socially salient items, the extent of distractor processing would still depend on the perceptual load of the task. They reasoned that if face processing is automatic and mandatory (Farah et al., 1995; Kanwisher et al., 1997) then faces may continue to be effective distractors even when perceptual load is high.

Lavie et al. (2003) designed a task that involved searching for a name (target) among a list of non-words (non-target elements) while ignoring famous faces (distractors) that were presented at the side of the list. The target word was a name of a pop star or politician and the participant was asked to classify the name and press a button to indicate their choice. The faces presented could either be congruent (e.g. the face of a pop star presented concurrently with the name of a pop star) or incongruent (e.g. the face of a pop star presented with the name of a politician) with equal probability. The perceptual load of the task was manipulated by altering the number of non-words flanking the target (see figure 6.1). 
Figure 6.1 Example of stimuli used by Lavie et al, 2003.

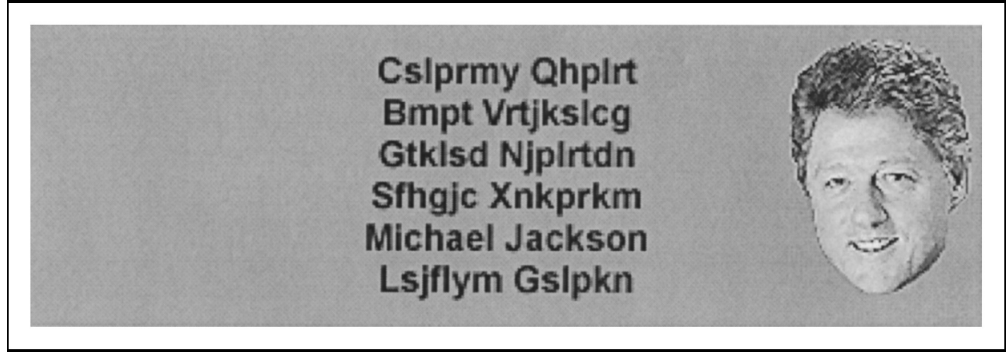

This is an example of a high perceptual load, incongruent trial

The results of this study demonstrated that, unlike the task with the letter distractors (Lavie, 1995and see experiment 1 in this thesis), there was an effect of congruency at all set sizes. This indicated that the faces were being processed at all levels of perceptual load. The hypothesis that faces hold a special status in attention was reinforced by a second study that employed the same paradigm but participants were asked to classify names of fruits and musical instruments. The results with these meaningful non-face items were similar to those found when using the original letter stimuli - the distractor processing was eliminated at high levels of perceptual load (Lavie et al., 2003).

Within this thesis, the behaviour of individuals with ASD on a task of selective attention and perceptual load with face distractor stimuli was investigated. The relevant literature on face processing will now be outlined, before discussing the issue of face processing abnormalities in ASD. Given that the central concern within this experiment is the question of whether individuals with ASD prioritise faces in the same way as typical adults, the bulk of the literature discussed will focus on the debate regarding whether faces are indeed a 'special' class of visual stimuli and what implications this has for ASD. 


\section{Face Processing in Typical Individuals}

\section{The Development of Face Processing Abilities}

In typical individuals, face processing is evident early in life. The propensity to look at faces in preference to other visual stimuli is evident nine minutes after birth (Goren et al., 1975; Johnson et al., 1991; Valenza et al., 1996). There are two main hypotheses regarding the mechanism underlying this seemingly innate ability. The 'structural hypothesis' (Johnson \& Morton, 1991) argues that babies are born with a reflex-like mechanism based on an inborn representation of the human face that contains information regarding the configuration of the main facial elements. They assert that there are two sub-cortical systems: 'Conspec', a primitive and experience-independent mechanism that causes infants to preferentially attend to faces, and 'Conlern', a system that is modified by visual input to allow competent processing of faces leading to recognition and discrimination. In contrast, the 'sensoric hypothesis' (Banks \& Salapatek, 1981; Kleiner \& Banks, 1987) refutes the claim that there is any innate knowledge of faces and states that babies prefer faces because the physical properties of faces are perfectly matched to the sensoric abilities of newborns. Hence, faces are highly interesting compared to other visual patterns. A set of experiments by Valenza and colleagues (Valenza et al., 1996) examined the respective roles that the structural information and distribution of spatial frequencies play in face preference. They showed that even when the distribution of spatial frequencies was optimal, the structural information was the factor that determined the infants' preference, thus providing evidence in favour of the structural hypothesis.

Though very young infants already have remarkable face processing skills, these abilities develop throughout childhood. It has been demonstrated that 
performance on a task of face recognition increases between the ages of 2 and 10 years (Blaney \& Winograd, 1978; Carey et al., 1980; Carey \& Diamond, 1977; Chung \& Thomson, 1995; Flin, 1980; Schwarzer, 2000). The methods of identification also evolve. Two to three year olds analyse single features and focus alternately on different features, five year olds consistently focus on a specific feature and, from age six to adulthood, there is a shift from analytical to holistic processing (Carey \& Diamond, 1994; Tanaka et al., 1998). It seems that during childhood, the single facial features become integrated into the face as a whole; allowing better categorisation and recognition. This shift may be due to the vast number of facial representations that are acquired - leading children to discover that holistic processing is the best method to facilitate differentiation between the many different faces that they encounter. This dependence on configural, rather than featural, information in adulthood was clearly demonstrated by Young, Hellawell and Hay (1987) who constructed 'chimeric' face stimuli by joining the top and bottom halves of different famous faces (see figure 6.2). They found that when the halves were aligned, participants found it hard to name the identity of the two individuals. Conversely, when the halves were misaligned, identification was a great deal easier. It is thought that when the two halves are aligned, they produce a new configuration which is processed holistically and therefore undermines the ability to extract the information from the separate halves that is necessary for recognition of the famous faces. 
Figure 6.2 Example of a chimeric face of Johnny Depp and Tom Cruise.

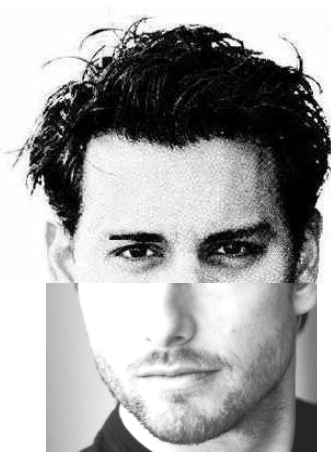

6.2a: aligned face

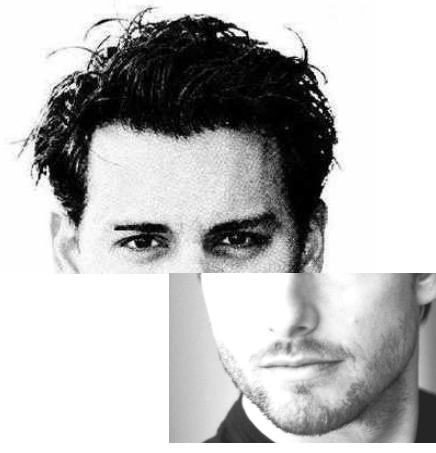

6.2b: misaligned face

\section{Are Faces Special?}

Much of the research into the cognitive neuroscience of face processing is devoted to one central question: is face processing carried out by specialised domainspecific modules or by domain-general systems that can also process non-face visual stimuli? Studies from behavioural, neuropsychology, neuroimaging and neurophysiology research have addressed this issue.

At the behavioural level, demonstration of an inversion effect appears to suggest domain specificity. Yin(1969) showed that inverting stimuli hinders the identification of faces to a greater degree than is seen with objects. It also appears that by inverting a face, one is forced to use a featural style of analysis rather than the configural process that is generally applied to upright faces. The 'Thatcher Illusion', where the face is inverted but the eyes and mouth remain upright, is clear evidence of this. The altered face seems almost normal until it is rotated to its canonical orientation, at which point it appears grotesque and extremely altered (Thompson, 1980). This inversion effect is generally taken as evidence that faces engage mechanisms that are not used when processing non-face stimuli. 
Compelling evidence for a specific face processing system is found within neuropsychological literature regarding deficits in face processing. Prosopagnosia is a syndrome where the ability to recognise and identify previously familiar faces is compromised, yet the ability to recognise objects is preserved (Bodamer, 1947). Prosopagnosia most commonly occurs after neurological trauma (usually bilateral damage to the inferior aspect of the temporal cortex, in the region of the fusiform gyrus (Benton, 1980; Farah, 1990) but can also result from a developmental disorder. Moreover, a double dissociation is seen; Moskovitch, Wincour and Behrmann (1997) present $\mathrm{CK}$, an individual who demonstrated intact face processing skills but whose ability to recognise objects was impaired. Such lesion studies also play a role in the debate, outlined above, regarding whether any innate face processing ability exists in newborn infants. Farah and colleagues (Farah et al., 2000) report the case of Adam, a 16 year old boy who, following bilateral infarction in the occipital lobes one day after birth, displayed prosopagnosia. The selective impairment in face recognition led the authors to conclude that this case was evidence for an innate face processing mechanism which cannot be compensated for by other neural structures - despite the fact that there had been many years to develop alternative strategies.

The domain-specific hypothesis is also supported by neuroimaging and electrophysiological ERP studies that have shown a difference in the responses elicited by face and non-face stimuli in typical individuals. Positron emission tomography (PET) and fMRI studies have highlighted a region in the fusiform gyrus of the extra-striate visual cortex (usually on the right side) that is activated in response to faces - and is activated at least twice as strongly for face stimuli as it is for objects (Haxby et al., 1994; Kanwisher et al., 1997; Kanwisher, 2000; McCarthy et al., 1997; Tong et al., 2000). Termed the 'fusiform face area' (FFA), there is a 
great deal of evidence that this is the primary locus of face processing. In support of this, Vignal and colleagues (Vignal et al., 2000) demonstrated that direct electrical stimulation of the right anterior inferior frontal gyrus can lead to hallucinations of faces.

Using electrophysiological studies, Jeffreys and Tukmachi (1992) found that an early positive ERP (P190) is elicited in response to faces, whereas the responses to non-face stimuli were smaller and later. A negative peak (N200) that appears to be specific to face processing has also been identified - and is localised over regions of the inferior occipito-temporal cortex (Allison et al., 1994). This is consistent with the areas of activation observed in PET and fMRI studies.

The nature of this proposed specific neural system has also been explored. Bruce and Young (1986) suggest that the system has multiple components and may be subdivided into subsystems (underpinned by different neural loci) that are responsible for various tasks involved in face processing. They emphasise the distinction between processes that work on the recognition of identity, and those that work on recognition of expression and speech-related movement. This distinction is backed up by studies that seem to show that recognition of identity and expression can proceed independently (Ellis et al., 1990; Young et al., 1986b). These studies showed that familiarity and repetition priming facilitates face identity processing but not face-expression processing. In line with this componential hypothesis, ERP studies have identified a marker of face processing called the N170 (Bentin et al., 1996) and shown that while this response is unaffected by the familiarity of the face presented, later ERPs (250ms to $500 \mathrm{~ms}$ after stimulus onset) are influenced by familiarity. Furthermore, an imaging study by Moscovitch and colleagues (Moscovitch et al., 1997) revealed a right anterior region on the border of the 
fusiform and parahippocampal gyri that is activated in response to face recognition but not when only simple face discrimination is required. These findings suggest that there is an early visual analysis phase followed by recognition mechanisms.

Aside from the FFA, additional neural regions have also been linked to face processing. fMRI and intracranial recordings have shown face-related activation in right posterior lateral temporal areas around the superior temporal sulcus (STS) (Haxby et al., 2000; Pelphrey et al., 2003; Puce et al., 1998). This area is suggested to play a role in eye-gaze processing (Puce et al., 1998; Wicker et al., 1998), extracting socially relevant information such as theory of mind judgments (Fletcher et al., 1995; Gallagher et al., 2000) intention (Allison et al., 2000; Frith \& Frith, 1999) and trustworthiness (Winston et al., 2002). The anterior middle temporal gyrus and the orbitofrontal cortex have also been implicated: there is increased activation in response to famous and personally familiar faces (Gorno-Tempini et al., 1998; Gorno-Tempini \& Price, 2001; Nakamura et al., 2000; Sergent \& Signoret, 1992).

The amygdala, insula, limbic stystem, sensori-motor cortex and inferior frontal cortex (IFC) are also thought to play a role in the emotional evaluation of facial expressions (Johnson, 2005; Nakamura et al., 1999). Both the STS and the IFC belong to the mirror neuron system, a system that generates internal representations of the actions performed by oneself or others (Gallese et al., 2004).

Taken together, these neuropsychological, imaging and electrophysiological studies provide a convincing argument for a specific distributed neural system that underlies face processing. Proponents of this idea go on to suggest that this specific face processing system is subject to its own capacity limits (Boutet \& Chaudhuri, 2001; Palermo \& Rhodes, 2002). Jenkins et al. (Jenkins et al., 2003) showed that distraction from face distractors could only be reduced by adding an additional face 
(rather than extra non-face stimuli) - indicating that face processing capacity is not filled by non-face items. This may explain how the face distractors in Lavie's perceptual load study (Lavie et al., 2003) were able to be processed at even the high levels of perceptual load.

However, the debate about faces being special is far from over. It has been suggested that rather than the FFA being a module devoted to face processing, it is responsible for 'expert-level' categorisation (Gauthier et al., 1999; Gauthier et al., 2000; Tarr \& Gauthier, 2000). It may be that our vast exposure to, and hence extensive knowledge of, faces that leads to an extremely high level of expertise that sets faces apart from other visual stimuli. Studies have shown that the fusiform gyrus is activated when bird and car experts view examples from within their category of expertise (Gauthier et al., 2000). With this hypothesis in mind, however, the question still remains as to why alphanumeric stimuli (for which we have a level of expertise similar to that for faces) fail to activate the proposed face-selective regions. Furthermore, Duchaine et al. (Duchaine et al., 2004) were able to successfully train a patient with severe developmental prosopagnosia to obtain a high level of expertise in the recognition and discrimination of 'Greebles' (face like stimuli) - suggesting that Greeble and face recognition rely on separate mechanisms. It seems that, at present, the evidence indicates that domain-specific accounts are more likely.

\section{Faces processing as a mandatory and automatic process}

Linked to the idea of a specialised neural network is the concept that faces are processed automatically. Fodor (1983) initially described specialised modules as a system characterised by the fact that it responds in an automatic and mandatory manner whenever the triggering input is presented. Subsequently, neuropsychologists 
have argued that the dedicated face module responds whenever a face is presented (Allison et al., 1995; Farah et al., 1995; Puce et al., 1996). However, Wojciulik et al. (Wojciulik et al., 1998) showed that the neural responses were somewhat modulated by attention; with less FFA activation recorded when the stimuli were presented in the periphery.

The apparent automaticity of face processing means that, for typically developing individuals, faces seem to capture attention more above and beyond other visual stimuli (Ro et al., 2001; Theeuwes \& Van der Stigchel, 2006). This, together with the idea of a separate capacity, seems to explain why individuals taking part in Lavie's selective attention study (Lavie et al., 2003) were unable to ignore distractor faces even when they were irrelevant and may have hindered task performance (Jenkins et al., 2002; Lavie et al., 2003; Young et al., 1986a).

Within this chapter, we explore whether this automatic and mandatory processing of faces is evident in ASD. In order to approach this issue, the existing literature on face processing deficits in ASD will be examined.

\section{Face Processing in ASD}

A deficit in face processing is commonly highlighted as a component of the impaired social behaviours that are displayed by individuals with ASD. As mentioned above, it is thought to result from a dysfunction of face processing neural regions such as the fusiform gyrus (Dawson et al., 2005) or amygdala (Baron-Cohen et al., 2000) and/or a lack of interest in social stimuli early in life (Dawson et al., 2005; Grelotti et al., 2002; Schultz, 2005). Parental reports and retrospective analysis of home videos indicate poor eye contact, delayed, reduced or absent gaze-following, 
reduced orienting to faces, lack of responsiveness to parents' voice and lack of spontaneous imitation (see Jemel et al., 2006).

\section{Behavioural Studies}

Behavioural techniques have been used to investigate a number of aspects of face processing within ASD and findings have highlighted problems with recognition of previously familiar faces (Boucher et al., 1998), matching facial expressions (Celani et al., 1999; Gepner et al., 2001) and immediate recognition of novel faces (Boucher \& Lewis, 1992; Hauck et al., 1998). Klin et al. (1999) found a deficit in face recognition in children with ASD when compared to both typical children and children with pervasive developmental disorder not otherwise specified (PDD-NOS) - even when the groups were matched on both non-verbal and verbal mental age. This led the authors to conclude that the observed abnormalities in ASD cannot be due to verbal or non-verbal deficits, task demands or visual memory deficits. While such studies propose the presence of an absolute deficit, others suggest that though face processing is abnormal and may well involve altered mechanisms, performance can be at normal levels. In an influential study, Langdell (1978) asked participants to identify the partially obscured faces of their peers. He varied which facial areas were masked and showed that the children with autism were poorer than controls when the lower regions were obscured but outperformed controls when the upper face regions were obscured. Therefore, despite there being no overall difference in task accuracy, there was an altered pattern of correct responses. This finding may indicate that individuals with ASD have a deficit in configural face processing; relying more heavily on featural part-based encoding. This possibility is backed up by the observation that the inversion effect is not as strong, or is absent, in ASD. Hobson et 
al. (Hobson et al., 1988) showed that adolescents with ASD were superior to controls in identifying expression and identity of upside down faces and Tantam et al. showed an absence of the inversion effect (Tantam et al., 1989). More recent studies, however, have questioned these findings and have produced evidence of a typical face inversion effect in ASD (Joseph \& Tanaka, 2003; Lahaie et al., 2006; Teunisse \& de Gelder, 2003) and a typical response to the Thatcher illusion (Rouse et al., 2004). Other studies show that ASD individuals are capable of extracting the holistic representation of faces (Joseph \& Tanaka, 2003; Lopez et al., 2004). Lopez and colleagues used cued and non-cued tasks of face matching that involved both wholeface and part-face stimuli. In the cued condition, both the group of adolescents with ASD and the control group showed a face recognition advantage after viewing face features in context rather than isolation, and showed an effect of configural cueing. In the non-cued condition, however, the individuals with ASD failed to process the faces holistically. These results go against the suggestion that there is an overall deficit in holistic processing in ASD (e.g. Mottron \& Belleville, 1993) and rather conclude that holistic processing can be elicited under appropriate testing conditions.

\section{Neuroimaging and electrophysiological Studies}

Given that several neural correlates of face processing have been suggested, neuroimaging is a powerful tool with which to investigate face processing in ASD.

Schultz and colleagues were the first to use neuroimaging studies to provide evidence of atypical face processing within this clinical population (Schultz et al., 2000). They scanned high functioning individuals with ASD and age and IQmatched control participants while they performed a within-category discrimination task that involved face and object stimuli. Overall, there was a different pattern of 
activation in response to the face stimuli in the ASD individuals when compared to the control group, whereas the pattern in response to objects was similar across all participants. In the face task, the individuals with ASD showed reduced fusiform gyrus activation and greater inferior temporal gyrus (ITG) activation. This may again be evidence that individuals with ASD have a tendency to use alternative part based featural strategies to process faces and, as a result, recruit neural regions that are usually used for non-face object perception in typical adults (Schultz et al., 2000). Three other studies have also reported similar reduced fusiform gyrus activation in ASD (Critchley et al., 2000; Hubl et al., 2003; Pierce et al., 2001). It is important to note, however, that unlike patients with fusiform damage (who also have reduced/ absent FFA activation), individuals with ASD are generally not prosopagnosic. The abnormal face processing behaviour seems to not simply be a reflection of a basic deficit in face identification.

Hadjikhani and colleagues (Hadjikhani et al., 2007) also observed differences in the activation levels of several areas belonging to the face perception network in ASD. These included parts of the mirror neuron system (the inferior frontal cortex and STS), the amygdala, somatosensory cortex (S1) and premotor cortex (PM). These regions are particularly associated with emotion perception. The mirror neuron system has been suggested to be the neural basis of 'mind reading' and empathy (Gallese, 2003; Leslie et al., 2004), and therefore it has been hypothesised that it is the basis of some of the social deficits that are seen in ASD (Williams et al., 2001; Williams et al., 2006). Both anatomical and functional abnormalities of the mirror neuron system have been recently highlighted in ASD (Dapretto et al., 2006; Hadjikhani et al., 2006). Given that strong correlations have been found between the STS and other areas of the face-processing network (amygdala, FFA, PM), it has also 
be hypothesised that face-processing is modulated by the mirror neuron system (Hadjikhani et al., 2007). Overall, it seems that although neuroimaging studies have shown that activation of areas involved in face processing can be found in ASD, this is generally accompanied by hypoactivation of other regions of the wider face perception system.

Electrophysiological studies have added to the literature regarding face processing in ASD. The differences in ERP responses to faces observed between ASD and control individuals have included delayed latency (McPartland et al., 2004; O'Connor et al., 2005), smaller amplitude of responses overall (Bailey et al., 2005; O'Connor et al., 2005), atypical distribution across the scalp (Bailey et al., 2005), an absence of right-hemisphere lateralisation (Dawson et al., 2005; McPartland et al., 2004; Senju et al., 2005), no influence of familiarity or expression on evoked responses (Dawson et al., 2002; Dawson et al., 2004b) and lack of a differential response to upright and inverted faces (Grice et al., 2001; McPartland et al., 2004).

The delayed N170 face-sensitive peak in ASD has been taken as evidence of a reduced speed of early structural encoding of faces (McPartland et al., 2004). O'Connor et al. (2005) looked at the N170, P1 and occipital P2 responses elicited by emotional face stimuli in children and adults with ASD. The results indicated longer latencies of P1 and N170 and smaller N170 amplitude in adults with ASD when compared to an age-matched control group but no difference between the groups of children.

The atypical scalp distribution has been used as evidence of abnormal cortical specialisation for faces in ASD (Dawson et al., 2005). Most noticeably, the absence of the right hemisphere lateralisation was highlighted. In contrast, however, a MEG 
study showed right-sided activation to faces in ASD during a categorisation and identification task (Bailey et al., 2005).

The absence of the inversion effect has also been indicated by Grice et al, (2001) who reported no difference in the spectral gamma responses (oscillations in EEG within a 30-70 $\mathrm{Hz}$ frequency band) to inverted and upright faces.

\section{Gaze Fixation}

Investigation of gaze patterns has been used to shed light on what seems to be an altered pattern of face processing. Abnormal gaze behaviour when viewing face stimuli, whether the face appears in isolation (Dalton et al., 2005), within a social scene picture (Riby \& Hancock, 2008) or movie extract (Klin et al., 2002; Speer et al., 2007) appears to be evident in ASD. While watching videos of social interaction between actors, individuals with ASD spent significantly less time fixating on the eye than the mouth region. They fixated equally on the eyes and bodies, and least on the surrounding objects. In contrast, the typical adults generally focussed on the eyes of the actors. The ASD individuals also fixated to a greater extent on 'unimportant' face regions such as an ear, chin or hairline, than on internal features such as the eyes, nose and mouth (Pelphrey et al., 2002). Klin and colleagues showed participants video sequences and found that individuals with ASD looked at the actors' mouths more than at their eyes while the opposite pattern was seen for control participants (Klin et al., 2002). Dalton et al. (2005) suggests that the differing pattern is due to a reduced interest in the eye region. They asked participants to perform tasks of face expression and familiarity judgements and found that while there was no difference in the amount of fixation to the mouth, the individuals with ASD spent less time looking at the eye region than the control participants. This lack of 
spontaneous gaze fixation towards the eyes has been echoed in a number of other studies (Dalton et al., 2005; Klin et al., 2002; Pelphrey et al., 2002; Riby \& Hancock, 2008). It may be the case, therefore, that the reduced FFA activation (cited above) is due to a failure to adequately inspect the areas of the face that are necessary for face identification and discrimination. Indeed, in the study by Hadjikhani et al. (2004) where a fixation point was used to ensure that all participants were attending to the stimuli in the same manner, the fusiform gyrus was activated normally in the individuals with ASD.

The abnormal gaze behaviour in ASD may well play a role in the proposed inability to interpret information from the faces of others - such as the observed deficits in joint-attention and following gaze cues (Swettenham et al., 1998).

\section{Emotion Processing}

Emotion processing in ASD has received a great deal of attention within the literature and a full review of the extensive literature on this topic is beyond the scope of the thesis. Instead, a brief outline is given in order to allow a complete picture of face processing deficits in ASD to be obtained. Gross (2004) and Hobson (1986a) have put forward the hypothesis that a problem with understanding others' emotion and an inability to perceive social cues in faces is the basis of the social deficit in ASD. Various studies have highlighted impairments in recognition and understanding of facial expressions in ASD (Celani et al., 1999; Gross, 2004; Hobson, 1986a; Hobson, 1986b; Tantam et al., 1989). Low-functioning children with

autism were shown to have a difficulty matching photographs and videos of emotional faces with appropriate drawings of the facial expressions (Celani et al., 1999; Deruelle et al., 2004). More specifically, Pelphrey et al.(2002) found that the deficits were primarily evident in the processing of fearful faces, while Adolphs and 
colleagues (Adolphs et al., 2001) showed that judgement of trustworthiness and approachability proved the most challenging. Conversely, a number of studies have shown intact face expression processing in ASD (Castelli, 2005; Grossman et al., 2000; Pelphrey et al., 2002). When participants were matched on verbal IQ, the deficit disappeared. Ozonoff et al.(Ozonoff et al., 1990) showed that the ability to sort and match emotional faces was equivalent to that of control children when the groups were matched on verbal mental age. This suggests that the problems previously reported may have been due to the verbal demands of a task of emotion recognition. Many researchers have also failed to find any impairment in the recognition of basic emotions (Baron-Cohen et al., 1993; Baron-Cohen et al., 1997; Grossman et al., 2000).

\section{Aetiology of abnormal face processing in ASD}

When considering a possible cause of the altered face processing behaviour in $\mathrm{ASD}$, there is a debate regarding the extent to which environment contributes to the face processing impairment and whether or not there is an innate deficit. For example, it could be that an innate lack of prioritisation of attention for face stimuli leads to less experience looking at faces, and consequently less specialisation of the face-processing mechanism. Researchers have analysed the early home videos of infants and found that the children who were later diagnosed with autism failed to attend to faces (Baranek, 1999; Osterling \& Dawson, 1994). Moreover, Osterling et al. (Osterling et al., 2002) used a similar methodology to show that, at one year of age, infants who were later diagnosed with autism could even be differentiated from infants with mental retardation on the basis of how much time they spent looking at other people. In favour of the hypothesis that it is this lack of attention to faces in 
ASD that could lead to a later impairment is the 'experience expectant' model of face processing by Nelson (2001). This theory proposes that humans are born with a neural system that is predisposed to specialise in face processing, but that only through exposure to faces does this specialisation occur. If the system does not receive the necessary input, then there may be no development of a specific face processing network (Marcus \& Nelson, 2001). It has also been suggested that early infancy is a sensitive period during which this experience of faces must occur in order to set up the neural mechanisms necessary for future face processing ability. Pascalis, De Hann and Nelson (2002) examined the ability of 6-month olds, 9-month olds and adults to discriminate between pairs of human and monkey face photographs. They found that the youngest group could distinguish between both human and monkey face-pairs but by the age of nine months the ability to discriminate monkey faces had been lost. This indicates that without experience of the relevant social stimuli in early infancy, the ability to expertly process faces may not develop. This finding parallels the work regarding a critical period for language acquisition and the observation that the ability to distinguish between phonemes from foreign languages is lost if there is no relevant input in the months after birth. The importance of early exposure has also been demonstrated by Le Grand and colleagues (Le Grand et al., 2001) who measured featural and configural face processing in adolescents who, due to bilateral congenital cataracts, had been deprived of visual exposure to faces during the first few months of life. They demonstrated that, when compared to an age-matched control group, these participants were performing at normal levels on the tasks that required the processing of featural information but were significantly impaired on the tasks that involved processing configural face information. Crucially, this configural 
processing deficit was specific to faces - no problem was observed in encoding configural information present in geometric patterns. Le Grand et al's findings indicate that exposure to faces in early months of life is critical for an individual's propensity to process faces configurally. Given that a number of studies (outlined above) have highlighted a reduced tendency to process faces configurally in ASD, Le Grand's finding has particular implications for the question of altered face processing in ASD.

If a reduced attraction to faces during infancy in ASD is indeed responsible for the face processing deficits, the question still remains as to why this diminished interest exists. One suggestion is that individuals with ASD have an aversion to looking at the rapid motion that is inherent in faces. Grandin (1986) and Williams (1992), both authors with high-functioning ASD, have expressed that the darting movement of the eyes is particularly overwhelming for them to process. Consequently, the developing neural systems are denied the visual input that is necessary for long-term face processing competency (Sasson, 2006). It seems, therefore, that current literature supports a synthesis of the nature and nurture hypotheses outlined above: neural abnormalities may lead to the failure of individuals with ASD to pay preferential attention to faces, which in turn prevents the development of specialised face processing systems.

\section{Attention to social stimuli - are faces special in ASD?}

The most relevant question to this thesis is whether faces are 'special' within ASD. The behaviour shown by typical adults on tasks of selective attention and perceptual load in the presence of social stimuli (Lavie et al., 2003) demonstrates that face distractors are processed in an automatic and mandatory fashion - and capture 
the attention even at the expense of central task performance. With respect to ASD, however, social attention appears to be altered. It has been suggested that individuals show an overly selective response to social stimuli (Schriebman \& Lovaas, 1973). More recently, Leekam and Moore (2001) have highlighted an impairment in exogenous orienting of attention to social cues but not to objects in individuals with ASD. In an fMRI study, Bird et al. (Bird et al., 2006) showed that there was a lack of attentional modulation of the neural responses to face stimuli in the ASD group (i.e. attention to the face did not enhance FFA activation levels). With house stimuli, however, attention modulated the neural response in house-selective areas for both participant groups. The authors hypothesised the absence of attentional modulation to social stimuli was due to weaker connectivity between V1 and extrastriate areas in ASD. As mentioned above, it has also been shown that individuals with ASD assign less of their attention to social stimuli, and instead show a preference for non-social stimuli (Dawson et al., 2004a; Klin et al., 2002; Swettenham et al., 1998). In this chapter, these suggestions of altered social attention in ASD will be further examined within the framework of the perceptual load theory of selective attention and cognitive control.

\section{Summary}

Though the evidence regarding a face processing in ASD is mixed, it seems clear that altered strategies and behaviours are employed by individuals with the condition. Crucially, there does not seem to be any evidence that individuals with ASD show the same prioritised attention for social stimuli. With respect to my own studies, it was predicted, therefore, that for individuals with ASD, the capacity for processing socially meaningless stimuli would be dissociable from the capacity to 
process social stimuli. In contrast to the neutral stimuli used in earlier studies, it was

predicted that the proposed increase in perceptual capacity within ASD (as suggested by the results of experiments one and two) would not confer an advantage with face stimuli. It was also predicted that the individuals with ASD would not show the automatic and mandatory processing of the distractor face stimuli that was evident in the typical group at high levels of perceptual load. As a result, it is expected that the individuals with ASD will show a reduced congruency effect of the distractor faces at the higher set sizes. This prediction will be explored in the following experiments.

\section{Experiment Five - The Effect of Non-Famous Faces as Distractors in Perceptual Load Tasks}

As discussed above, Lavie, Ro and Russell (2003) demonstrated that famous distractor faces are processed at all levels of perceptual load. While this is compelling evidence for the 'special' status of faces, it is possible that the distractor faces influenced attention specifically because they were familiar, famous faces. The familiarity of a face has been shown to affect the way it is processed and Rossion and colleagues (Rossion et al., 1999) have demonstrated this at a neural level. They examined event related potentials (ERPs) to face processing and found that while the N170 (a marker of face-specific processing) is unaffected by familiarity of the face, there are later markers (between $250 \mathrm{~ms}$ and $500 \mathrm{~ms}$ after stimulus onset) that do relate to familiarity. On a behavioural level it was also seen that individuals show faster visual search for their own face (Tong \& Nakayama, 1999).

In order to address this issue, the present study used a variation of the flanker task used by Lavie et al. (2003) but with anonymous faces. The effect of perceptual 
load on processing non-famous distractor faces could therefore be assessed.

Participants were asked to perform a central task that involved classifying names based on gender while ignoring male and female distractor faces.

\section{Methods}

Participants.

20 young adults between the ages of 21 and 29 years took part in the study. All had normal, or corrected to normal vision.

Table 6.1 Descriptive Statistics for Participants

\begin{tabular}{lccccc}
\hline Statistic & $\begin{array}{c}\text { Age } \\
\text { (years: } \\
\text { months) }\end{array}$ & $\begin{array}{c}\text { WASI } \\
\text { vocabulary } \\
\text { subtest }\end{array}$ & $\begin{array}{c}\text { WASI } \\
\text { matrix } \\
\text { reasoning }\end{array}$ & $\begin{array}{c}\text { WASI } \\
\text { full scale IQ } \\
\text { (2 subtests) }\end{array}$ \\
\hline$[\mathrm{n}=20]$ & mean & $26: 5$ & 65.7 & 58.8 & 122 \\
6 females & S.D & $2: 4$ & 6.9 & 6.1 & 10.4 \\
14 males & range & $21: 5-29: 9$ & $52-74$ & $47-67$ & $101-137$ \\
\hline
\end{tabular}

\section{Stimuli.}

As with the previous experiments, Visual Basic was used to create computer based stimuli that were presented on a custom built small form desktop computer and displayed on a ProLite 15 " flat LCD screen (1280 x 1024 pixel resolution, $2 \mathrm{~ms}$ response rate). Viewing distance was $60 \mathrm{~cm}$.

Stimuli were presented in black against a grey background. Following the presentation of a fixation cross in the centre of the screen for $500 \mathrm{~ms}$, a target name was presented in centre of the screen. In order to manipulate the perceptual load of the task, the name would be presented alone (set size one) or among a list of one, 
three or five non-words (set sizes two, four and six respectively). In each trial, a distractor face measuring $4.1^{\circ} \times 3.3^{\circ}$ visual angles was presented at the side of the list of words, $5^{\circ}$ from fixation. These distractor faces were either congruent (same sex as the target name) or incongruent (opposite sex as target name). Four blocks of 192 trials were created with each set size and distractor condition appearing equally. For set sizes two and four, the position of the target within the list was counterbalanced. A set of six male and six female forenames were used as the target words and a set of twelve non-words were used as the non-target elements. The names and non-words were matched in length and varied between four and seven letters (see appendix II).

Figure 6.3 Examples of stimuli used in experiment five

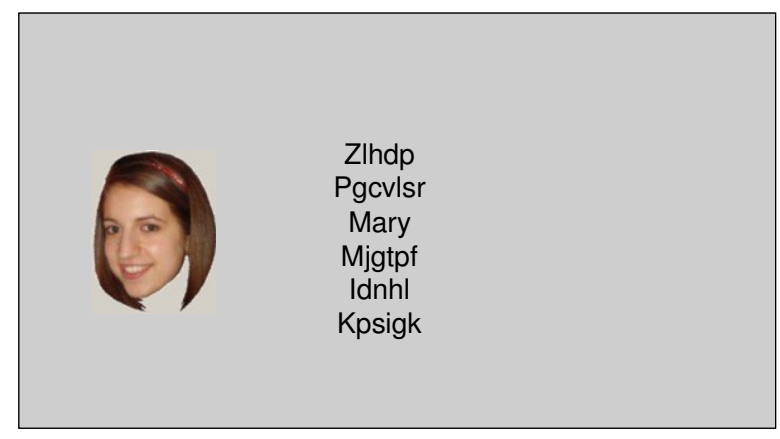

Example of a compatible trial with high perceptual load.

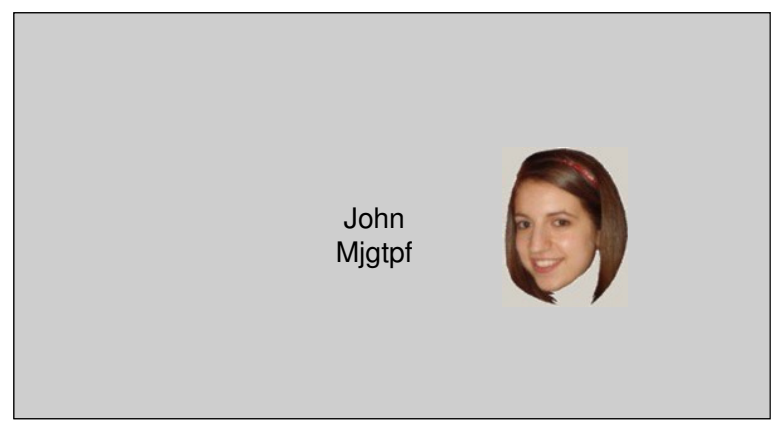

Example of an incongruent trial with low perceptual load. 


\section{Procedure.}

Participants were instructed to ignore the faces and to indicate whether the name presented was male or female by pressing a key ( $\mathrm{X}$ or $\mathrm{M}$ respectively). The relevant buttons were labelled accordingly. Following a set of five practice trials, with feedback, the participants completed the four experimental blocks and were able to take breaks between blocks if required. Every response was followed by a $1000 \mathrm{~ms}$ inter-trial interval and incorrect responses elicited a brief computer tone. The accuracy and response time for each trial was recorded by the computer program and subsequent comparison of reaction times for the two distractor trial types within high and low load conditions allowed the influence of distractors to be ascertained (see figure 6.4).

Figure 6.4 Time course of experimental trials.

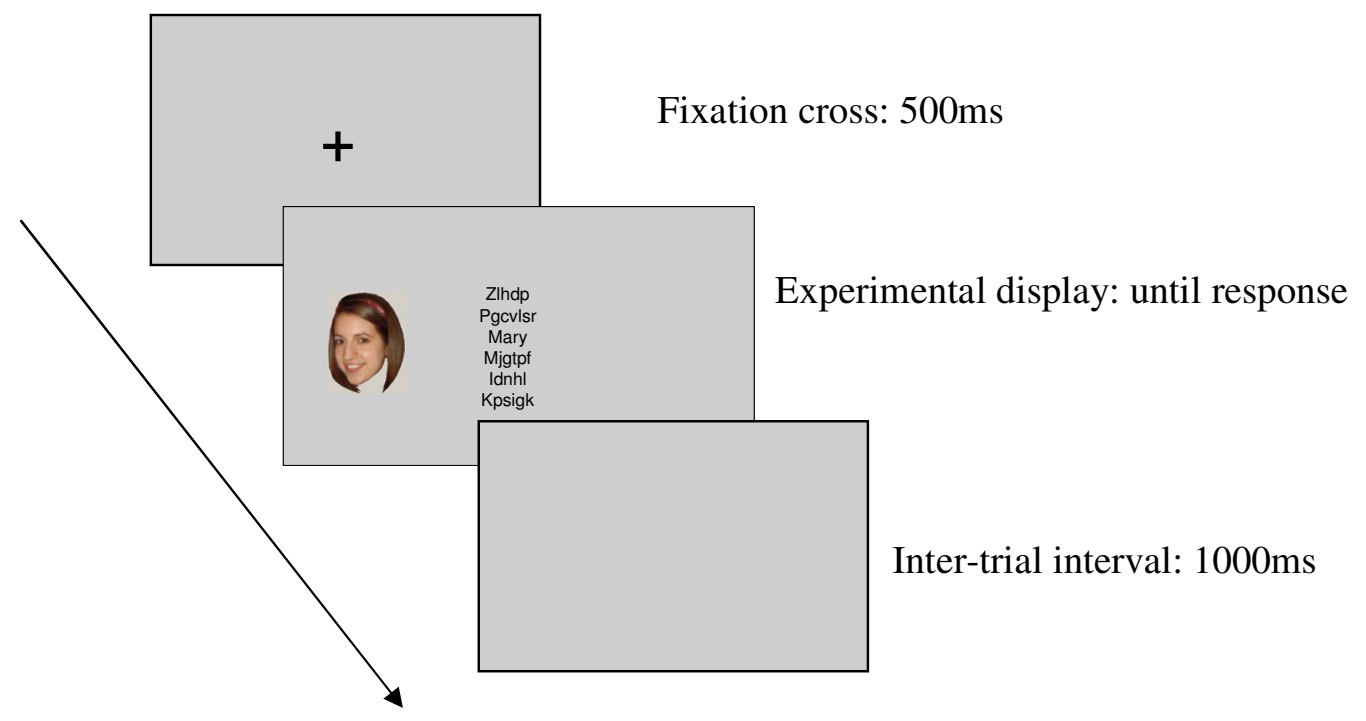

\section{Results}

For each participant, the median reaction times for correct responses to congruent and incongruent trials at each set size were calculated (see table 6.2). 
Table 6.2 Overall mean median RTs (ms) and standard deviations (SD) under congruent (cong.) and incongruent (incong.) distractor conditions at each set size

\begin{tabular}{|c|c|c|c|c|}
\hline & $\frac{\text { Set size } 1}{\text { cong incong. }}$ & $\frac{\text { Set size } 2}{\text { cong incong. }}$ & $\frac{\text { Set size } 4}{\text { cong incong. }}$ & $\frac{\text { Set size 6 }}{\text { cong incong. }}$ \\
\hline $\begin{array}{l}\text { Mean RT } \\
\text { (SD) }\end{array}$ & $\begin{array}{cc}569 & 596 \\
(50) & (59)\end{array}$ & $\begin{array}{r}634 \\
(58)\end{array}$ & $\begin{array}{rr}826 & 869 \\
(101) & (117)\end{array}$ & $\begin{array}{cc}1064 & 1098 \\
(122) & (113)\end{array}$ \\
\hline
\end{tabular}

A repeated measures ANOVA was then performed on these values. There was a main effect of set size $\left(F(3,57)=369.05, p<0.001, \eta_{p}{ }^{2}=0.95\right)$ and a main effect of distractor congruency $\left(F(1,19)=13.95, p=0.001, \eta_{p}{ }^{2}=0.42\right)$. Inspection of these results revealed that the significant effects reflect the fact that reaction times increased with set size (as the task became more difficult) and were longer for incongruent trials (see figure 6.5).

Figure 6.5 Median reaction times for each trial condition.

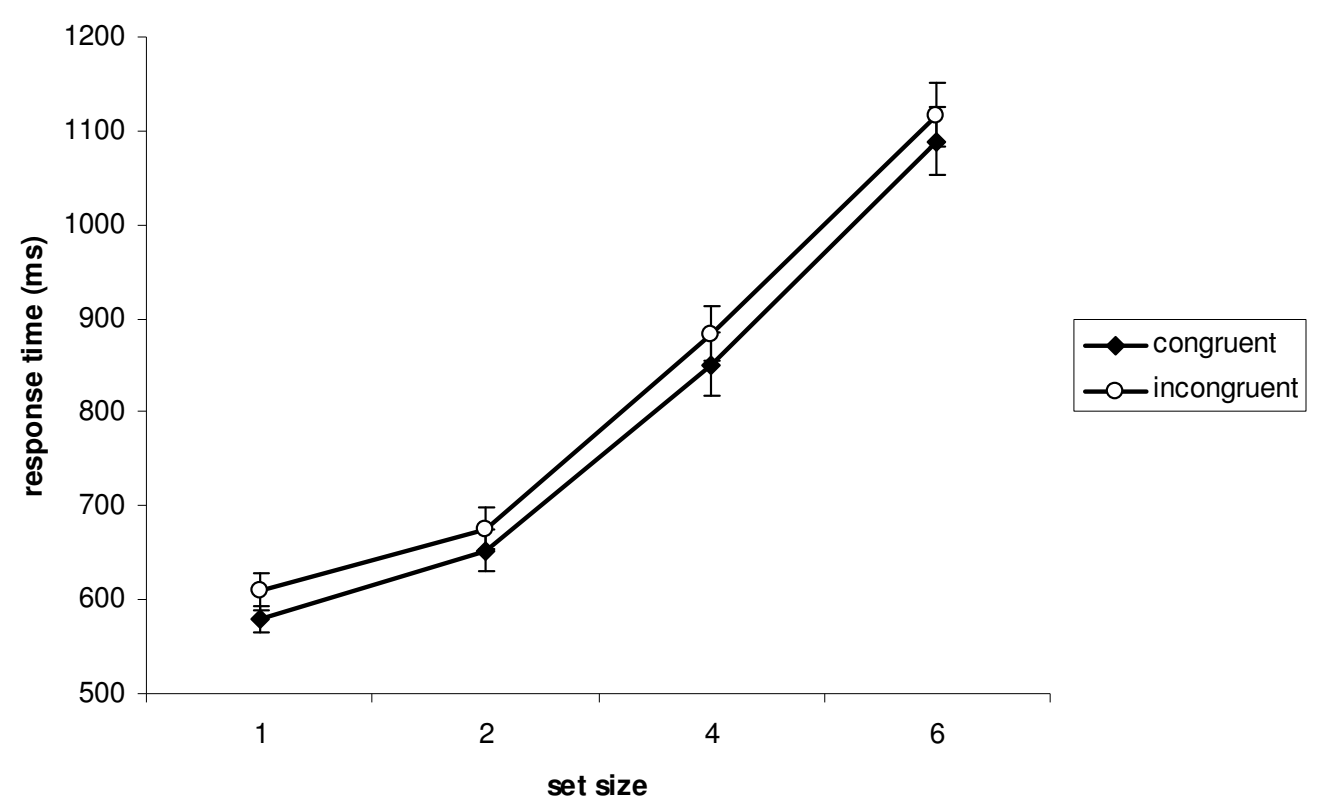


There was no interaction between set size and distractor congruency ( $\mathrm{F}$ $\left.(3,57)=0.40, \mathrm{p}=0.673, \eta_{\mathrm{p}}{ }^{2}=0.02\right)$ indicating that the congruency effect did not vary with set size (see figure 6.6).

Figure 6.6 Difference in reaction times between incongruent and congruent trials (congruency effect) for each set size.

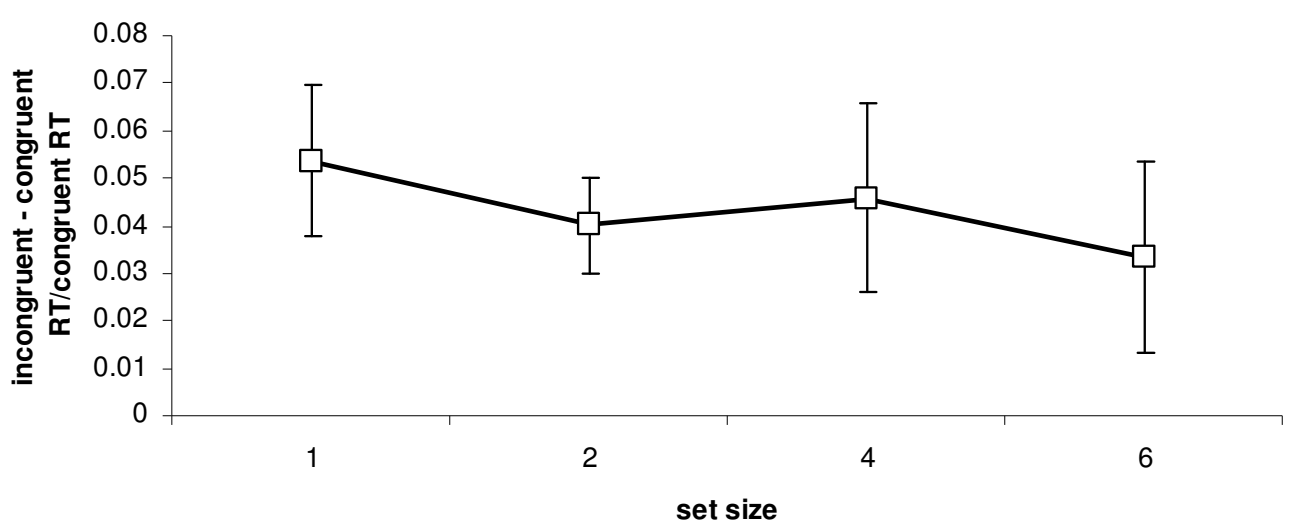

Post-hoc t-tests indicated that there was a significant congruency effect at all set sizes (set size one $(\mathrm{t}(19)=3.27, \mathrm{p}=0.004)$ set size two $(\mathrm{t}(19)=3.98, \mathrm{p}=0.001)$ set size four $(\mathrm{t}(19)=2.98, \mathrm{p}=0.008)$ set size $\operatorname{six}(\mathrm{t}(19)=2.48, \mathrm{p}=0.023))$.

\section{Discussion}

The results echo those of Lavie et al. (2003) where familiar famous faces were used. Our findings show that even with unfamiliar faces, distractor faces continued to be processed in high perceptual load situations.

This study has demonstrated that when anonymous faces are used as distractors in a flanker experiment, the extent to which they are processed is not modulated by the perceptual load of the central task. In even the high load conditions 
(set size four and six) there is a congruency effect - with incongruent trials eliciting longer response times than congruent trials. This confirms that the pattern of results seen by Lavie et al. (2003) was not simply an artefact of the use of famous faces. The data from this study also adds to the body of evidence indicating that faces are not processed in the same way as other stimuli. Given that previous research has shown that meaningful non-face distractor items do not affect reaction times at set sizes of four and six, it seems that irrelevant distractor faces are processed even in situations within which there would be no interference from non-face items.

The adaptation of the famous face flanker task into one involving anonymous faces also has useful implications. The task no longer has a world-knowledge component. This makes it more accessible to participants who may have a reduced level of world-knowledge. This task can therefore be used to assess the nature of face-processing with respect to perceptual load in individuals with ASD.

\section{Experiment Six - Faces as distractor elements in a task of selective attention with ASD.}

In the previous experiment, it was established that anonymous face distractors are processed at all levels of perceptual load in typical adults. This is similar to the findings by Lavie et al.(2003) concerning the processing of famous faces under various levels of perceptual load. Given that the new paradigm developed in Experiment five no longer has the world-knowledge component, and is therefore suitable for use with a clinical population, it was used to examine selective attention and perceptual load in ASD. Based on the observed social deficits within the condition, it was predicted that individuals with ASD would not show an increased 
capacity for face distractors when compared with control participants. This would be in contrast to the increased capacity for neutral stimuli that was highlighted in experiments one, two and three.

\section{Methods}

\section{Participants.}

16 young adults with ASD and 16 control adults took part in the study. Participants were matched on non-verbal IQ (WASI) and reading ability was verified using the National Adult Reading Test (NART).

Table 6.3 Descriptive Statistics for each Group

\begin{tabular}{llccccc}
\hline Group & Statistic & $\begin{array}{c}\text { Age } \\
\text { (years: } \\
\text { months) }\end{array}$ & $\begin{array}{c}\text { WASI } \\
\text { vocabulary } \\
\text { subtest }\end{array}$ & $\begin{array}{c}\text { WASI } \\
\text { matrix } \\
\text { reasoning }\end{array}$ & $\begin{array}{c}\text { WASI } \\
\text { full scale IQ } \\
\text { (2 subtests) }\end{array}$ & NART \\
\hline $\begin{array}{l}\text { ASD } \\
(\mathrm{n}=16)\end{array}$ & mean & $23: 8$ & 63.1 & 52.1 & 113 & 18.7 \\
10 males & S.D & $4: 0$ & 5.7 & 9.1 & 12.0 & 5.8 \\
6 females & range & $18: 8-33: 7$ & $54-72$ & $37-67$ & $95-136$ & $10-28$ \\
\hline $\begin{array}{l}\text { Control } \\
\text { (n=16) }\end{array}$ & mean & $26: 8$ & 64.6 & 57.9 & 120 & 15.7 \\
11 males & S.D & $2: 5$ & 7.4 & 6.6 & 11.6 & 6.4 \\
5 females & range & $21: 5-29: 9$ & $52-74$ & $41-67$ & $101-138$ & $7-27$ \\
\hline
\end{tabular}

Independent samples t-tests showed that the WASI and NART scores of the two groups were not significantly different (all $\mathrm{p}$ values $>0.05$ ).

\section{Stimuli and Procedure}

The stimuli and procedure were identical to those used in Experiment five. 


\section{Results}

The median correct RT and error rates for each distractor condition at set size one, two, four and six were calculated. All incorrect trials were excluded from further analyses. For each participant, the median reaction times for correct responses to congruent and incongruent trials at each set size were calculated (see table 6.4)

Table 6.4 Overall mean median RTs (ms) and standard deviations (SD) for the two groups under congruent (cong.) and incongruent (incong.) distractor conditions at each set size.

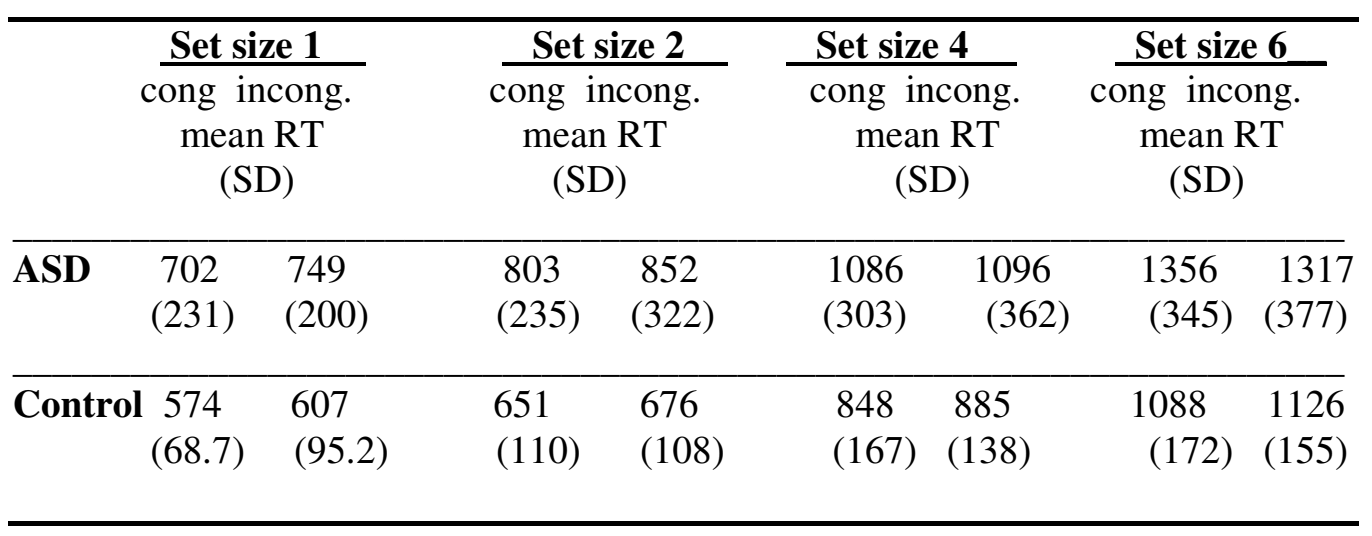

An analysis of variance (ANOVA) was performed on the mean median correct RTs with group (ASD vs. control) as the between-subjects factor and distractor congruency (congruent vs. incongruent) and set size (one, two, four \& six) as within-subject factors.

There was a main effect of set size $\left(F(3,90)=369.69, p<0.001, \eta_{\mathrm{p}}{ }^{2}=0.93\right)$ and a main effect of distractor congruency $\left(F(1,30)=11.94, p=0.002, \eta_{p}^{2}=0.29\right)$. Inspection of these results revealed that reaction times increased with set size (as the 
task became more difficult) and were longer for incongruent trials. The main effect of congruency did not interact with set size $\left(F(3,90)=2.03, p=0.116, \eta_{p}{ }^{2}=0.06\right.$ or group $\left(\mathrm{F}(3,90)=1.27, \mathrm{p}=0.268, \eta_{\mathrm{p}}{ }^{2}=0.04\right)$. There was also no significant interaction between set size and group $\left(F(3,90)=3.09, \mathrm{p}=0.063, \eta_{\mathrm{p}}{ }^{2}=0.09\right)$.

There was a main effect of group $\left(\mathrm{F}(1,30)=10.88, \mathrm{p}=0.003, \eta_{\mathrm{p}}{ }^{2}=0.27\right)$ due to generally slower reaction times of the ASD participants.

The three-way interaction between group, set size and distractor congruency was also significant $\left(\mathrm{F}(3,90)=3.22, \mathrm{p}=0.039, \eta_{\mathrm{p}}{ }^{2}=0.10\right)$ indicating that the two groups are showing different congruency effects at the various set sizes (see figure 6.7).

Figure 6.7 Congruency effects of each group at each set size

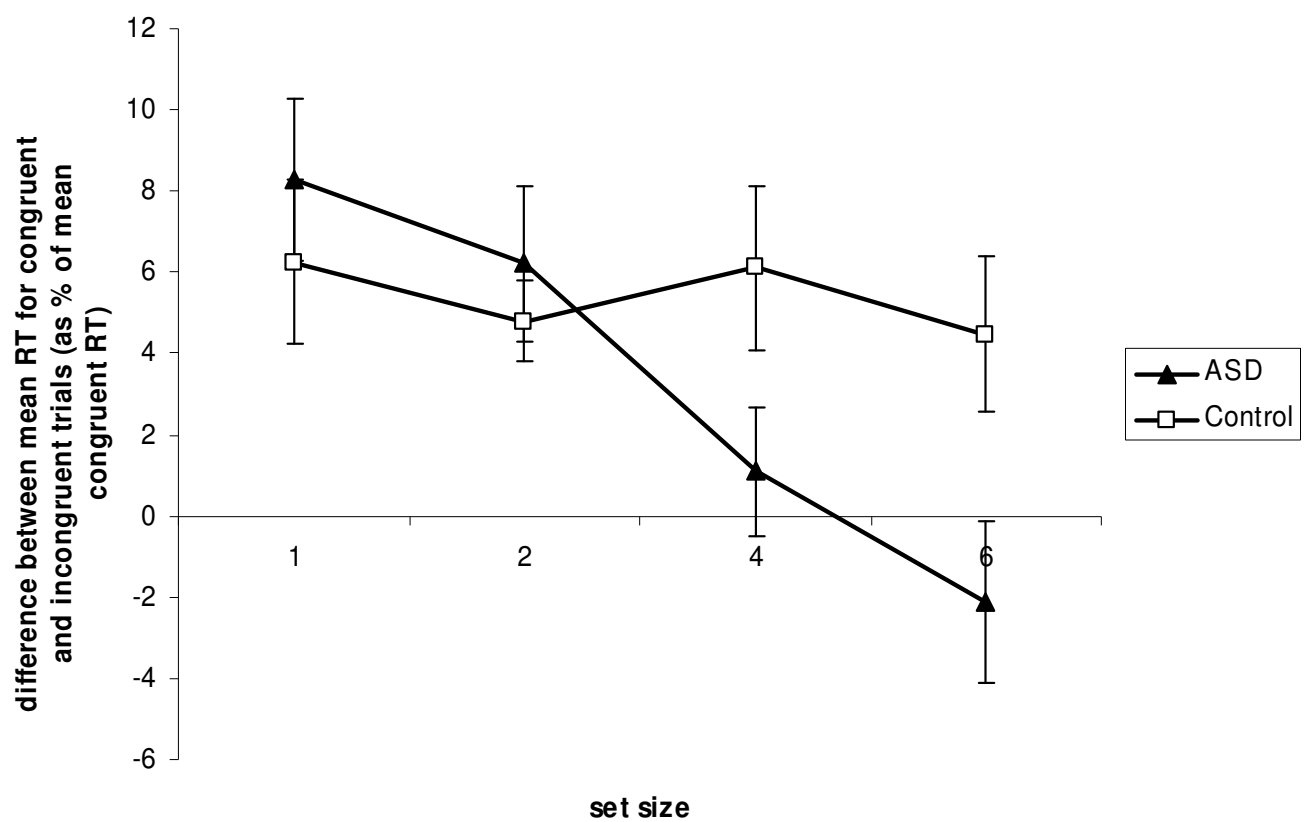

Post hoc t-tests were used to further investigate the significant three way interaction. In the control group, there was a significant effect of congruency at all 
set sizes (set size one $(\mathrm{t}(15)=3.06, \mathrm{p}=0.008)$ set size two $(\mathrm{t}(15)=4.32, \mathrm{p}=0.01)$ set size four $(\mathrm{t}(15)=2.5, \mathrm{p}=0.026)$ set size $\operatorname{six}(\mathrm{t}(15)=2.27, \mathrm{p}=0.039))$.

In the ASD group, there was significant effect of congruency at set size one $(\mathrm{t}(15)=3.97, \mathrm{p}=0.001)$ and two $(\mathrm{t}(15)=3.26, \mathrm{p}=0.005)$ but not for set size four $(\mathrm{t}(15)=0.68, \mathrm{p}=0.509)$ or $\operatorname{six}(\mathrm{t}(15)=-0.84, \mathrm{p}=0.414)$.

\section{Accuracy Data}

Analysis of variance was performed on the number of errors for each group across each condition (see table 6.5 for absolute values).

Table 6.5 Accuracy rates (proportion correct) and standard deviations (SD) for the two groups under congruent (cong.) and incongruent (incong.) distractor conditions at each set size.

\begin{tabular}{|c|c|c|c|c|}
\hline & Set size 1 & Set size 2 & Set size 4 & Set size 6 \\
\hline & $\begin{array}{l}\text { cong. incong. } \\
\text { Accuracy } \\
\text { (SD) }\end{array}$ & $\begin{array}{l}\text { cong. incong. } \\
\text { Accuracy } \\
\text { (SD) }\end{array}$ & $\begin{array}{l}\text { cong. incong. } \\
\text { Accuracy } \\
\text { (SD) }\end{array}$ & $\begin{array}{l}\text { cong. incong. } \\
\text { Accuracy } \\
\text { (SD) }\end{array}$ \\
\hline ASD & $\begin{array}{cc}0.948 & 0.943 \\
(0.047) & (0.052)\end{array}$ & $\begin{array}{rr}0.948 & 0.945 \\
(0.054) & (0.069)\end{array}$ & $\begin{array}{cc}0.979 & 0.956 \\
(0.043) & (0.058)\end{array}$ & $\begin{array}{rr}0.956 & 0.971 \\
(0.044) & (0.039)\end{array}$ \\
\hline Control & $\begin{array}{rr}0.927 & 0.945 \\
(0.076) & (0.045)\end{array}$ & $\begin{array}{cc}0.956 & 0.919 \\
(0.041) & (0.086)\end{array}$ & $\begin{array}{rr}0.948 & 0.943 \\
(0.047) & (0.037)\end{array}$ & $\begin{array}{cc}0.961 & 0.948 \\
(0.057) & (0.202)\end{array}$ \\
\hline
\end{tabular}

There were no significant main effects or interactions between the error rates for both groups under each condition (all $\mathrm{p}$ values $>0.05$ ). The error rates are consistently low across all conditions for both participants with ASD and control participants. 


\section{Discussion}

The results from this experiment show that, in a task involving selective attention and perceptual load, the impact of distractor faces is very different for typical adults and adults with ASD. Whereas typical adults continue to be distracted at all set sizes, ASD individuals show the same pattern with face distractors as was seen with neutral letter distractors in experiment one. When the perceptual load of the central task is increased, the individuals with ASD seem not to be processing the face distractors; there is no congruency effect observed.

It appears, however, that the participants with ASD are not ignoring the faces entirely. They show an effect of distractor congruency on response times at low set sizes but this effect disappears at the higher set sizes. The implications of these results will be discussed in the overall chapter discussion.

\section{Experiment Seven - Selective Attention and Perceptual Load with Musical Instrument Distractors.}

In order to truly determine whether any a 'special' response is seen to face distractors at various levels of perceptual load, the results from the previous experiment must be compared to data from an equivalent task involving meaningful non-face distractors. The paradigm in experiment one (using neutral letter distractors) is not sufficient for this purpose as the task demands and procedure were very different from those used in experiment six. Instead, a control task identical in structure to experiments five and six but that uses pictures of musical instruments was employed. Participants were asked to classify the name of an instrument (string or wind) while ignoring pictures of the instruments that flanked the words. By using photographs of musical instruments, the distractors are meaningful, of similar visual 
complexity to faces and require subordinate category discrimination. In this way, the impact of face stimuli on selective attention can be more accurately ascertained.

\section{Methods}

\section{Participants}

14 young adults with ASD and 14 control adults took part in the study. Participants were matched on non-verbal IQ (WASI) and reading ability was verified using the National Adult Reading Test (NART) (see table 6.6).

Table 6.6 Descriptive Statistics for each Group

\begin{tabular}{lllcccc}
\hline Group & Statistic & $\begin{array}{c}\text { Age } \\
\text { (years: } \\
\text { months) }\end{array}$ & $\begin{array}{c}\text { WASI } \\
\text { vocabulary } \\
\text { subtest }\end{array}$ & $\begin{array}{c}\text { WASI } \\
\text { matrix } \\
\text { reasoning }\end{array}$ & $\begin{array}{c}\text { WASI } \\
\text { full scale IQ } \\
\text { (2 subtests) }\end{array}$ & NART \\
\hline $\begin{array}{l}\text { ASD } \\
(\mathrm{n}=14)\end{array}$ & mean & $24: 8$ & 64.4 & 54.4 & 117 & 16.6 \\
11 males & S.D & $4: 9$ & 6.9 & 10.5 & 14.9 & 5.5 \\
3 females & range & $19: 8-33: 7$ & $54-76$ & $37-67$ & $95-138$ & $8-25$ \\
\hline $\begin{array}{l}\text { Control } \\
\text { (n=16) }\end{array}$ & mean & $26: 1$ & 65.0 & 52.1 & 116 & 15.7 \\
10 males & S.D & $2: 9$ & 6.7 & 8.2 & 11.3 & 6.9 \\
4 females & range & $21: 5-31: 2$ & $59-71$ & $41-65$ & $101-133$ & $7-27$ \\
\hline
\end{tabular}

Independent samples t-tests verified that there was no difference between the groups on these measures (all $\mathrm{p}$ values $>0.4$ ).

\section{Stimuli and Procedure}

The experimental paradigm was similar to that of experiment six, but the male and female names and face distractors were replaced by the names of wind or 
stringed musical instruments and corresponding pictures. Stimuli were presented in black against a grey background. Following the presentation of a fixation cross in the centre of the screen for $500 \mathrm{~ms}$, then name of a target instrument was presented in centre of the screen. The target could be one of six wind instruments (clarinet, horn, saxophone, trombone, trumpet, tuba) or one of six stringed instruments (banjo, bass, cello, guitar, harp, violin). In order to manipulate the perceptual load of the task, the name would be presented alone (set size one) or among a list of one, three or five non-words (set sizes two, four and six respectively). A set of twelve non-words were used as the non-target elements. The names and non-words were matched in length. In each trial, a distractor instrument measuring $4.1^{\circ}$ x $3.3^{\circ}$ visual angles (identical size to face distractor from experiment six) was presented at the side of the list of words, $5^{\circ}$ from fixation. These distractor instruments were either congruent (same instrument class as the target name) or incongruent (opposite instrument class to the target name). Four blocks of 192 trials were created with each set size and distractor condition appearing equally. For set sizes two and four, the position of the target within the list was counterbalanced (see figure 6.8). Following the completion of the experimental trails, the pictures of the instruments were presented to each participant and they were asked to both name the instrument and indicate which group (string or wind) it belonged to. This was to ensure that all participants had the relevant knowledge to meaningfully complete the task (see appendix III full set of experimental stimuli). 
Figure 6.8 Example of stimuli used in Experiment seven

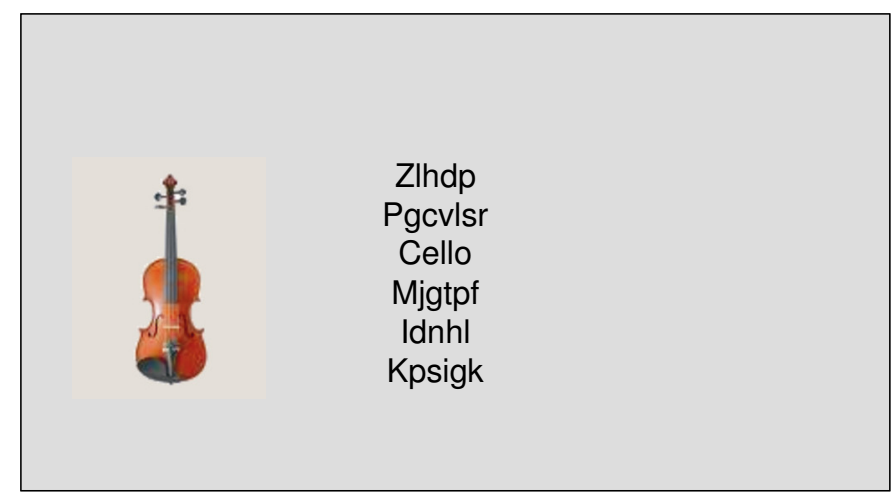

Example of a congruent trial with high perceptual load

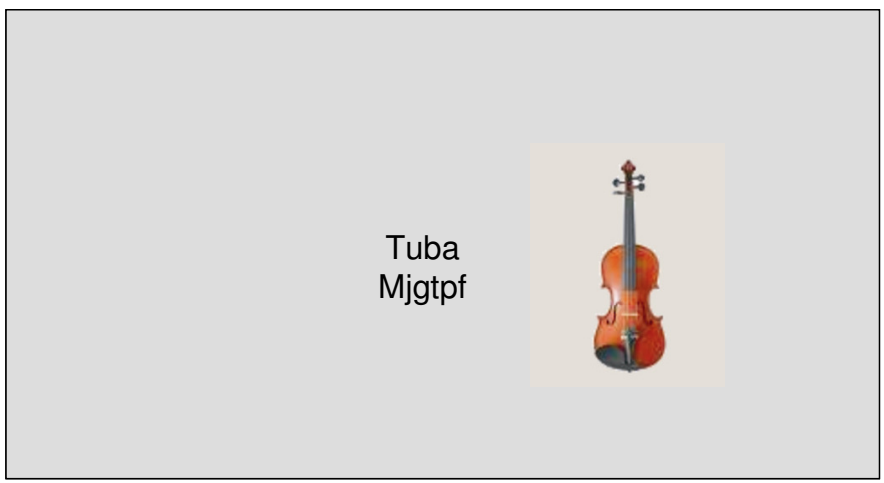

Example of an incongruent trial with low perceptual load

\section{Results}

The median correct RT and error rates for each distractor condition at set size one, two, four and six were calculated. All incorrect trials were excluded from further analyses. For each participant, the median reaction times for correct responses to congruent and incongruent trials at each set size were calculated (see table 6.7) 
Table 6.7 Overall mean RTs (ms) and standard deviations (SD) for the two groups under congruent (cong.) and incongruent (incong.) distractor conditions at each set size.

\begin{tabular}{|c|c|c|c|c|c|c|c|c|}
\hline \multicolumn{3}{|c|}{$\begin{array}{c}\text { Set size 1 } \\
\text { cong incong. } \\
\text { mean RT } \\
(\mathrm{SD})\end{array}$} & \multicolumn{2}{|c|}{$\begin{array}{c}\text { Set size 2 } \\
\text { cong incong. } \\
\text { mean RT } \\
\text { (SD) }\end{array}$} & \multicolumn{2}{|c|}{$\begin{array}{l}\text { Set size } 4 \\
\text { cong incong. } \\
\text { mean RT } \\
\text { (SD) }\end{array}$} & \multicolumn{2}{|c|}{$\begin{array}{l}\text { Set size 6 } \\
\text { cong incong. } \\
\text { mean RT } \\
\text { (SD) }\end{array}$} \\
\hline ASD & $\begin{array}{r}730 \\
(103)\end{array}$ & $\begin{array}{c}827 \\
(107)\end{array}$ & $\begin{array}{l}897 \\
(211)\end{array}$ & $\begin{array}{c}924 \\
(159)\end{array}$ & $\begin{array}{l}1154 \\
(272)\end{array}$ & $\begin{array}{l}1151 \\
(196)\end{array}$ & $\begin{array}{c}1420 \\
(273)\end{array}$ & $\begin{array}{l}1436 \\
(253)\end{array}$ \\
\hline Control & $\begin{array}{l}\text { I } 731 \\
(143)\end{array}$ & $\begin{array}{r}841 \\
(206)\end{array}$ & $\begin{array}{c}822 \\
(139)\end{array}$ & $\begin{array}{c}856 \\
(139)\end{array}$ & $\begin{array}{l}1060 \\
(170)\end{array}$ & $\begin{array}{l}1109 \\
(198)\end{array}$ & $\begin{array}{l}1344 \\
(205)\end{array}$ & $\begin{array}{r}1382 \\
(244)\end{array}$ \\
\hline
\end{tabular}

An analysis of variance (ANOVA) was performed on the median correct RTs with group (ASD vs. control) as the between-subjects factor and distractor congruency (congruent vs. incongruent) and set size (one, two, four \& six) as withinsubject factors.

There was a main effect of set size $\left(F(3,84)=322.50, p<0.001, \eta_{p}^{2}=0.92\right)$ and a main effect of distractor congruency $\left(\mathrm{F}(1,28)=31.88, \mathrm{p}<0.001, \eta_{\mathrm{p}}{ }^{2}=0.53\right)$. These significant effects reflect the fact that reaction times increased with set size (as the task became more difficult) and were longer for incongruent trials. The main effect of congruency did not interact with group $\left(F(3,84)=0.18, p=0.671, \eta_{p}{ }^{2}=0.01\right)$. There was also no significant interaction between set size and group $(F(3,84)=0.94$, $\left.\mathrm{p}=0.427, \eta_{\mathrm{p}}{ }^{2}=0.03\right)$. There was, however, a significant interaction between set size and congruency $\left(\mathrm{F}(3,84)=3.11, \mathrm{p}=0.031, \eta_{\mathrm{p}}{ }^{2}=0.10\right)$. As in experiment one, this is a reflection of the fact that there is a congruency effect at low set sizes (when the perceptual load of the task is low) but not at higher set sizes. 
There was no main effect of group $\left(\mathrm{F}(1,28)=0.76, \mathrm{p}=0.392, \eta_{\mathrm{p}}{ }^{2}=0.03\right)$; overall, both groups were performing the task at a similar speed.

The three-way interaction between group, set size and distractor congruency was not significant $\left(\mathrm{F}(3,84)=0.05, \mathrm{p}=0.984, \eta_{\mathrm{p}}{ }^{2}<0.01\right)$ indicating that the two groups are showing the same pattern of congruency effects at the various set sizes (see figure 6.9).

Figure 6.9 Graph of congruency effects for each group at each set size

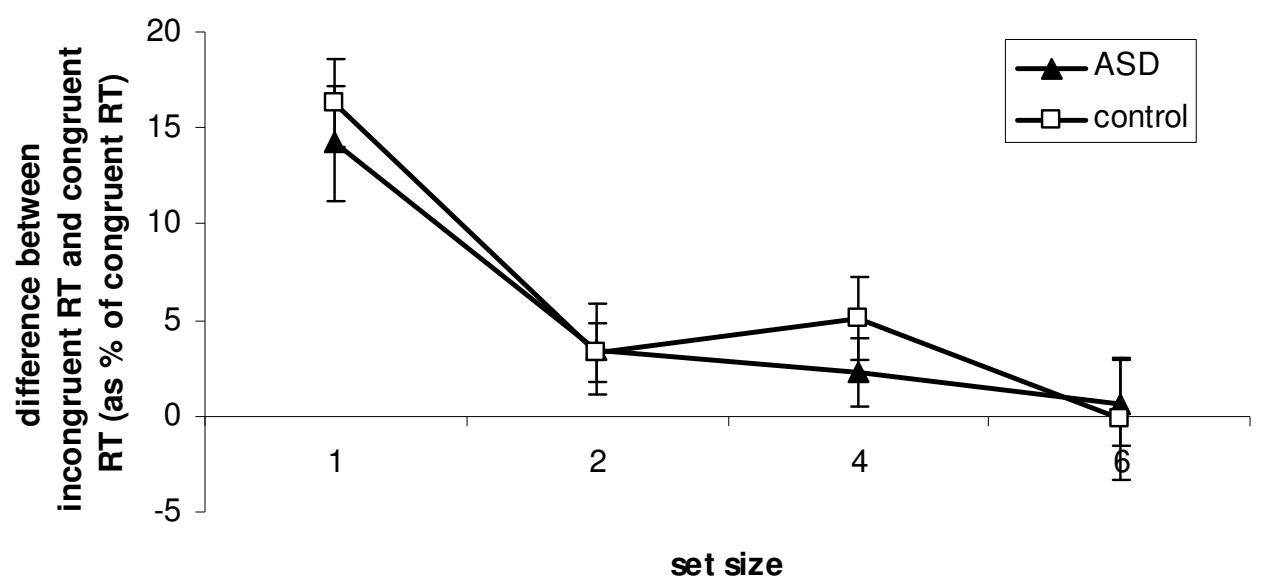

It appears that both groups display an effect of distractor type at the lower set sizes, which then disappears as the perceptual load of the task increased.

\section{Accuracy Data}

Analysis of variance was performed on the number of errors for each group across each condition (see table 6.8 for absolute values). 
Table 6.8 Accuracy rates (proportion correct) and standard deviations (SD) for the two groups under congruent (cong.) and incongruent (incong.) distractor conditions at each set size.

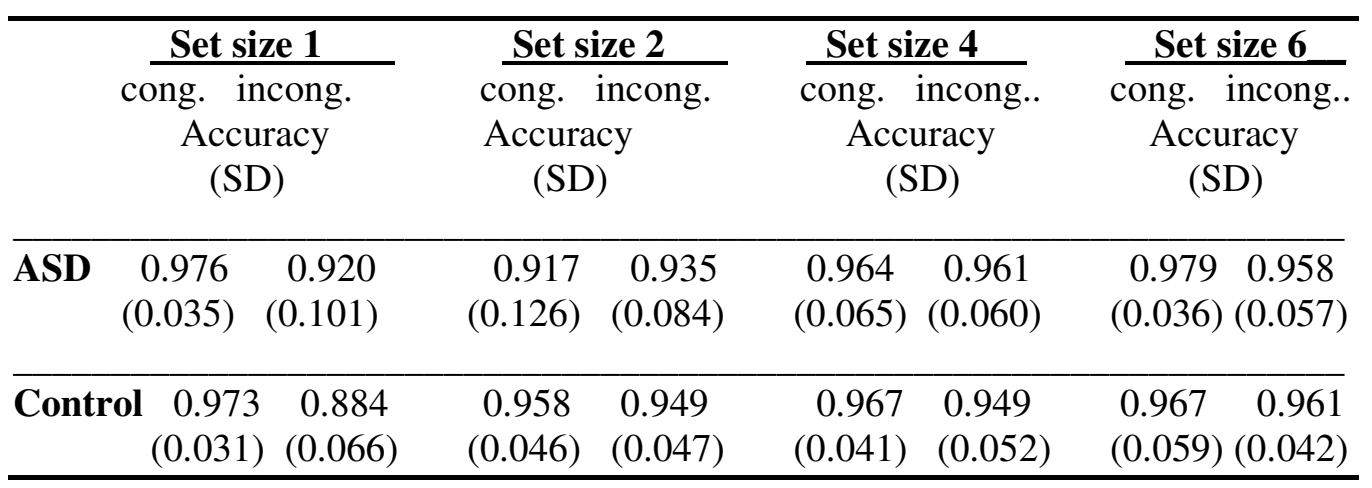

An ANOVA was performed on the accuracy data and revealed a main effect of set size $\left(\mathrm{F}(3,78)=5.83, \mathrm{p}=0.001, \eta_{\mathrm{p}}{ }^{2}=0.18\right)$ and a main effect of distractor congruency $\left(F(1,26)=9.43, p=0.005, \eta_{p}^{2}=0.27\right)$. Inspection of these results revealed that the error rates were higher for the lower set sizes and for incongruent trials. The latter observation is logical, while the idea of more errors being made at lower set sizes is a little counterintuitive. It may be that because the incongruent distractors are being processed at the lower set sizes, they are negatively influencing the responses. There was a significant interaction between congruency and set size $(\mathrm{F}(3,78)=7.90$, $\left.\mathrm{p}<0.001, \eta_{\mathrm{p}}{ }^{2}=0.23\right)$. In line with the reasoning regarding the main effect of set size, this reflects a greater number of errors in the incongruent trials vs. congruent trials at the lower set sizes. There was no significant interaction between set size and group $\left(\mathrm{F}(3,78)=2.85, \mathrm{p}=0.055, \eta_{\mathrm{p}}{ }^{2}=0.10\right.$ or between congruency and group $(\mathrm{F}(1,26)=0.98$, $\left.\mathrm{p}=0.331, \eta_{\mathrm{p}}^{2}=0.04\right)$. 
There was no main effect of group $\left(\mathrm{F}(1,26)<0.01, \mathrm{p}>0.999, \eta_{\mathrm{p}}{ }^{2}<0.01\right)$ due to generally slower reaction times of the ASD participants.

The three-way interaction between group, set size and distractor congruency was not significant $\left(\mathrm{F}(3,78)=0.76, \mathrm{p}=0.520, \eta_{\mathrm{p}}{ }^{2}=0.03\right)$ indicating that the two groups are not showing a different pattern of error rates across the various conditions.

\section{Comparing distractor interference from face and instrument distractors}

The results from this last experiment can be displayed along side the results from experiment six to illustrate the relative performance on the tasks involving face and musical instrument distractors. For each group (ASD and control) the results from the 16 individuals who participated in experiment six (face distractor task) and the results from the 14 individuals who participated in experiment seven (instrument distractor task) were compared.

ASD group

The behaviour of the ASD group was similar for both the face and musical instrument distractor tasks. There was an effect of both distractor types at the lower set sizes but not at the high set sizes (see figure 6.10). 
Figure 6.10 Graph to show congruency effect of face and instrument distractors in ASD group.

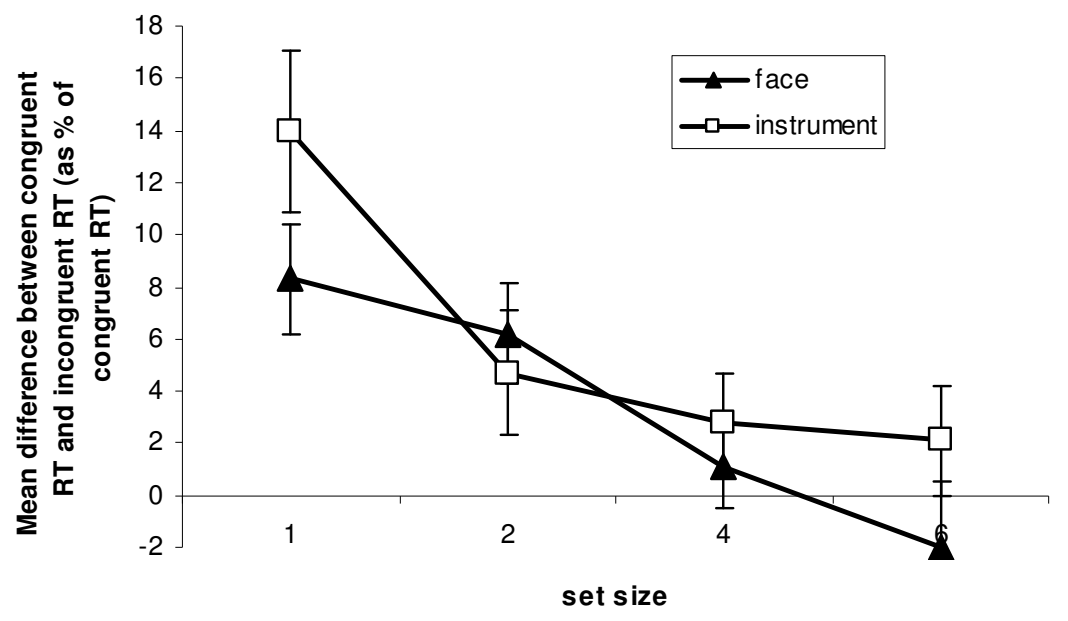

It appears from the graph (and the analysis of the previous experiments) that the behaviour of the participants with ASD is similar for both the face and instrument tasks. In order to confirm this statistically, an ANOVA was performed on the median correct RTs with task (face vs. instrument) as the between-subjects factor and distractor congruency (congruent vs. incongruent) and set size (one, two, four \& six) as within-subject factors.

There was a main effect of set size $\left(F(3,84)=260.30, p<0.001, \eta_{\mathrm{p}}{ }^{2}=0.90\right)$ and a main effect of distractor congruency $\left(F(1,28)=11.70, p=0.002, \eta_{p}^{2}=0.30\right)$. Inspection of these results revealed that reaction times increased with set size (as the task became more difficult) and were longer for incongruent trials. There was a significant interaction between set size and congruency $\left(F(3,84)=4.34, p=0.007, \eta_{p}^{2}\right.$ $=0.13)$. This reflects the fact that there was an effect of distractor congruency at the lower set sizes but not at the higher ones. There was no significant interaction between set size and task $\left(\mathrm{F}(3,84)=0.49, \mathrm{p}=0.691, \eta_{\mathrm{p}}{ }^{2}=0.02\right.$, or between congruency and task $\left(\mathrm{F}(3,84)=2.27, \mathrm{p}=0.143, \eta_{\mathrm{p}}{ }^{2}=0.08\right)$. 
There was no main effect of task $\left(F(1,28)=0.47, p=0.501, \eta_{p}{ }^{2}=0.02\right)$ due to generally slower reaction times of the ASD participants.

The three-way interaction between group, set size and distractor congruency was also not significant $\left(\mathrm{F}(3,84)=1.38, \mathrm{p}=0.254, \eta_{\mathrm{p}}{ }^{2}=0.05\right)$ confirming that the ASD participants are not showing different congruency effects at the various set sizes on the two separate tasks.

\section{Control group}

Comparison of control group's performance on the face and instrument distractor tasks highlights the markedly different behaviour that in seen in response to these two classes of stimuli (See figure 6.11). There is a congruency effect across all set sizes when faces are used as distractors, but when musical instruments were used the congruency effect that was present at low set sizes was eliminated as the perceptual load of the task increased.

Figure 6.11 Graph of congruency effects seen in the control group for face and instrument distractor tasks. 


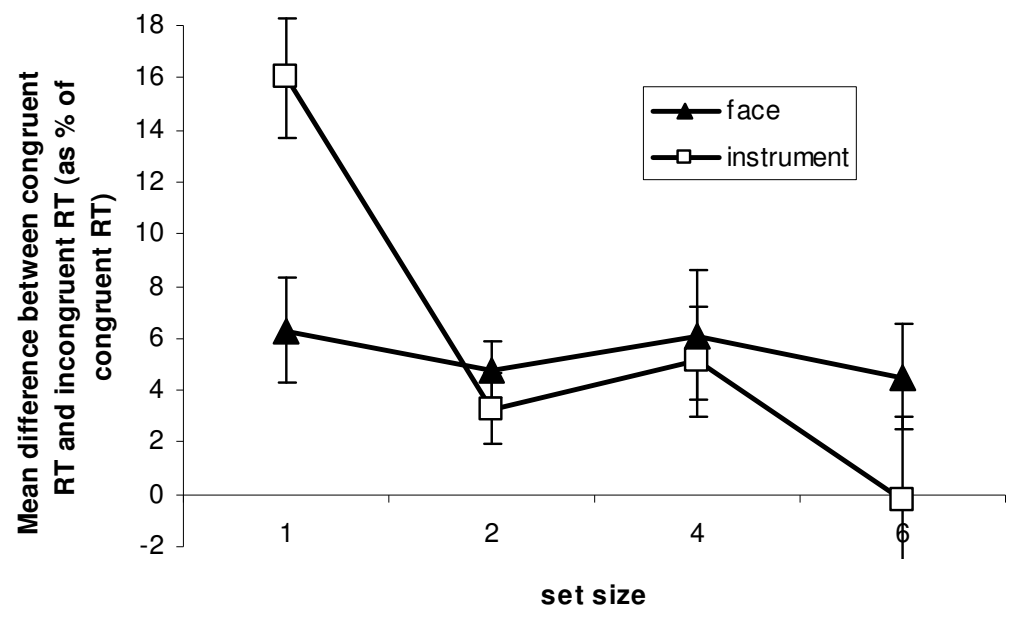

As with the ASD group, an ANOVA was performed on the median correct RTs with task (face vs. instrument) as the between-subjects factor and distractor congruency (congruent vs. incongruent) and set size (one, two, four \& six) as withinsubject factors.

There was a main effect of set size $\left(\mathrm{F}(3,87)=581.60, \mathrm{p}<0.001, \eta_{\mathrm{p}}{ }^{2}=0.95\right)$ and a main effect of distractor congruency $\left(F(1,29)=28.00, \mathrm{p}<0.001, \eta_{\mathrm{p}}{ }^{2}=0.49\right)$. Overall, for both face and instrument tasks, the reaction times increased with set size (as the task became more difficult) and were longer for incongruent trials. There was a significant interaction between set size and task $\left(\mathrm{F}(3,87)=3.57, \mathrm{p}=0.017, \eta_{\mathrm{p}}{ }^{2}=0.11\right.$ or group $\left(\mathrm{F}(3,90)=1.27, \mathrm{p}=0.268, \eta_{\mathrm{p}}^{2}=0.04\right)$. There was also a significant interaction between set size and distractor congruency $\left(\mathrm{F}(3,87)=3.71, \mathrm{p}=0.015, \eta_{\mathrm{p}}{ }^{2}=\right.$ 0.11). Overall, across the tasks there was a greater congruency effect at the lower set sizes. There was no interaction between congruency and task $(F(3,87)=0.62$, $\mathrm{p}=0.436, \eta_{\mathrm{p}}^{2}=0.02$ ); incongruent trials were responded to more slowly than the congruent trials in both tasks. 
There was a main effect of task type $\left(F(1,29)=32.35, \mathrm{p}<0.001, \eta_{\mathrm{p}}{ }^{2}=0.53\right)$. Inspection of the means showed that this is due to the response times on the instrument task being longer than those on the face task.

Crucially, the three-way interaction between group, set size and distractor congruency was also significant $\left(\mathrm{F}(3,87)=4.27, \mathrm{p}=0.007, \eta_{\mathrm{p}}{ }^{2}=0.13\right)$. This reflects what can be seen on the graph (see figure 6.11) and indicates that there is a significantly different pattern of congruency across the various set sizes on the two tasks.

Post hoc ANOVA indicated that this three-way interaction is due to the fact that there is an interaction between set size and congruency in the instrument task $(\mathrm{F}$ $(3,39)=4.28, \mathrm{p}=0.011, \eta_{\mathrm{p}}{ }^{2}=0.25$ but not in the face task $(\mathrm{F}(3,48)=0.68, \mathrm{p}=0.569$, $\left.\eta_{\mathrm{p}}^{2}=0.04\right)$

\section{Discussion}

The findings from this experiment, where pictures of meaningful non-social stimuli were used as distractor stimuli, provide crucial information that can be used to reference behaviour on tasks involving social stimuli. On the musical instrument task the typical adults showed an effect of distractor congruency that was modulated by perceptual load of the central task. While the pictures were processed at the lower set sizes, they appear to be ignored as the perceptual load of the task increased. This pattern of responses was also seen in the ASD group, where individuals showed a distractor congruency effect that was extinguished at higher set sizes.

The real value of this data, however, is as a control baseline with which to contrast the results from experiment six (face distractor task). A distinct difference is seen between the behaviour of the typical participants on the face and instrument 
tasks. On the latter, individuals seem able to disregard the distractors when the perceptual load of the task is sufficiently high. Conversely, the face distractors are processed - and impact on response times - irrespective of perceptual load level. This disparity was shown to be statistically significant.

The same distinction between the impact of social and non-social distractors is not seen within the ASD group. On both tasks, individuals with ASD seem to only process the distractor stimuli when the perceptual load of the central task is low. These findings will be fully discussed subsequently.

\section{Overall Discussion}

The results from the three experiments presented within this chapter can be used to draw a number of conclusions regarding the processing of social distractor stimuli. Firstly, it was confirmed that anonymous faces capture the attention of typical adults at all levels of perceptual load. This is consistent with the findings that Lavie and colleagues (Lavie et al., 2003) report concerning selective attention under various levels of perceptual load in the presence of famous face distractors.

Within this thesis, by using a paradigm with anonymous faces and asking participants to classify names based only on gender rather than identity, the worldknowledge component of the task was removed. This allows it to be used with a clinical population such as ASD where a typical level of world knowledge cannot be assumed.

In experiments 6 and 7, the performance of individuals with ASD and typical individuals on this task and a control task involving musical instrument distractors was examined. The results of the typical adults were similar to those reported by Lavie et al. (2003); the congruency effect of instrument distractors was eliminated at 
high levels of perceptual load whereas face distractors were processed at all set sizes. In stark contrast, the individuals with ASD showed the same pattern of results with both the face and instrument distractors. Both were processed at low set sizes but appear to be ignored as the perceptual load increased.

This finding provides evidence that faces do not capture attention in the same manner as for typical adults. In mainstream attention research, the inability to ignore distractor faces at high levels of load is used in support of the idea that face processing is an automatic and mandatory process and is not subject to general capacity limits (Lavie et al., 2003). The altered behaviour of the ASD group on the tasks presented here calls into question whether faces play a special role in attention for this clinical population. A distractor face, though irrelevant, may contain socially important information that is detrimental to ignore. The ability of individuals with ASD to disregard such stimuli clearly demonstrates the lack of importance that is placed on such details.

Interestingly, the faces are not completely ignored by the individuals with ASD; they are just treated in the same manner as any other non-social stimulus. This suggests that individuals with ASD are not averse to looking at faces entirely. One question that still remains, however, is whether the faces are being looked at, but are then discarded, or whether they are entirely disregarded at the higher set sizes. Unlike the brief presentation time in experiments one, two and three (where there was no time for voluntary eye movement), in experiments six and seven the stimuli remained on the screen for much longer. At the higher set sizes it may have been the case, therefore, either that the individuals with ASD did not fixate on the faces at all, or that they were looking at the faces but were then able to inhibit any impact that an incongruent face would have on the response time. If only the control individuals 
were looking at the faces at higher set sizes, one would expect that they would be slower to respond than the ASD individuals. This pattern was not seen; instead the participants in the ASD group were slower to respond at all the set sizes. This difference may, however, be due to problems with decision making or difficulty with the reading component of the task (although all participants reached typical levels on the National Adult Reading Test). To explore these issues, further research using eye-tracking equipment could be carried out to investigate to what extent the faces were fixated on by the individuals with ASD, and to identify any group differences in the gaze patterns. Additionally, it would be meaningful to carry out a similar experiment using fMRI. By asking participants to perform a selective attention task in the presence of flanking distractor faces within the scanner, the extent of FFA activation could be ascertained. This would indicate whether, and in which conditions, the distractor faces were being processed

The findings discussed thus far are in line with the original predictions made regarding the processing of distractor faces in ASD. Though it seems that there is evidence of an increased perceptual capacity (experiments one, two and three) it was hypothesized that not only would this not confer an advantage on a task that involved social stimuli, but that the propensity to process irrelevant face distractors would be reduced in comparison to typical controls.

It had also been expected, however, that on the task of non-social stimuli (musical instruments) there would be some evidence of the increased perceptual capacity in ASD; yet the results of experiment seven do not provide any indication of a significant difference in task performance between the two groups. There are a number of possible reasons for this. The most likely explanation concerns the task demands - which were very different from the earlier experiments that involved 
identification of a target letter. As raised above, the instrument task involved a reading component and, while all participants reached the required level on the National Adult Reading Test (Nelson, 1982), it is possible that this component placed disproportionately large cognitive demands on the ASD participants. This would have eradicated any superior performance that may have arisen due to increased capacity.

Overall, the results from experiments five, six and seven suggest that for individuals with ASD, faces do not capture attention in an automatic mandatory fashion. Both the social and non-social distractor items are treated in the same manner - and seem to be subject to the same capacity limits. Within mainstream attention literature, the observation that face distractors are processed irrespective of perceptual load (Lavie et al., 2003) has been taken as evidence for a separate processing capacity for faces. This is reinforced by the work of Jenkins and colleagues who demonstrate that the congruency effect of face distractors can only be diluted by the addition of other face stimuli and not by additional non-face items (Jenkins et al., 2003). It is possible that within ASD there is no such separate capacity, resulting in the observed impact of the perceptual load of the central task on the processing of the distractor faces. Alternatively, it may be that this separate capacity in typical adults is not innately assigned for faces but result from an interest in faces which leads to a great deal of experience and expertise (Gauthier \& Logothetis, 2000). The separate processing store may be reserved for a specialised interest, which in typical adults is social stimuli, but in ASD may be an object class such as trains or an ability to perform calculations or draw detailed pictures of visual scenes. This could explain the obsessive interests and savant abilities that are seen within the condition. 


\section{Chapter 7 - Discussion}

As highlighted in chapter two, there are many reports of altered attention as part of the behavioural profile of ASD. A review of the existing literature revealed a variety of findings concerning autism and selective attention. Studies have proposed abnormal levels of arousal (Dawson \& Lewy, 1989; Hutt et al., 1964; Ornitz \& Ritvo, 1968) and deficits in selectivity and filtering (Burack, 1994; Lovaas et al., 1979; Mann \& Walker, 2003; Rincover \& Ducharme, 1987). The nature of such abnormalities and deficits are, however, diverse and contradictory.

After studying mainstream attention research literature, the perceptual load theory of selective attention was identified as a key area for research. Within this thesis, the perceptual load theory of attention was applied to ASD in order to investigate whether this theory offers an explanation for the varying pattern of attentional behaviour seen in ASD. To date, there is no existing research on this area.

It was hypothesised that the pattern of distractor processing modulated by perceptual load that is seen in typical adults (Lavie, 1995) would be observed in ASD, but that within this clinical group, the impact of irrelevant distractors would persist at higher levels of perceptual load.

The first experiment in this thesis applied a response competition paradigm (Maylor \& Lavie, 1998) to assess selective attention at various levels of perceptual load. Participants were required to identify a target letter from within a ring of nontarget elements, while ignoring distractor letters that were presented offset from the central task. The results indicated, in line with the initial prediction, that the interference effect of the distractor stimuli was evident at higher levels of perceptual load in the ASD group. 
Given that there was no significant difference between the response times and accuracy rates of the two groups, it cannot be the case that the differing results are due to a general filtering deficit in ASD. An overall deficit of this nature which, irrespective of perceptual load level, prevents an individual from effectively assigning their attention to the central task would be accompanied by a drop in task performance (speed and/or accuracy).

This finding of increased distractor processing appears, therefore, to be evidence of increased perceptual capacity within ASD - i.e. when performing the central task the individuals with ASD have spare processing capacity which spills over to process the irrelevant distractors. Indeed, the extent of distractor processing has previously been used as an index of perceptual capacity in subgroups of the general population such as young children and the elderly (Huang-Pollock et al., 2002; Maylor \& Lavie, 1998).

This conclusion regarding increased perceptual capacity was based on the analysis of reaction time data which suggested that individuals with ASD were continuing to process flanking distractor stimuli at higher levels of perceptual load than control individuals. Measuring reaction times, however, is an indirect method of assessing attention and is unable to demonstrate whether the elements in question are actually being consciously perceived.

In order to address this issue, and to replicate the initial findings, the second study within this thesis employed a more direct measure to assess the impact of perceptual load on selective attention in ASD. In a dual-task paradigm, participants were required to both carry out a central letter-search task (of varying levels of perceptual load) and also to indicate the presence or absence of an expected critical stimulus. It was predicted that both the ASD and control groups would show a 
reduction in sensitivity to the critical stimulus as the perceptual load of the central task was increased, but that within the ASD group this reduction would occur at higher levels of load than for the typical adults. In the first of two experiments, the trials were presented in blocks of the same set size (i.e. same level of perceptual load). The results demonstrated that for the typical group the percentage of CS detection and perceptual sensitivity dropped as the level of perceptual load increased, whereas for the individuals with ASD these two measures seemed to remain constant across all levels of perceptual load. At the high levels of load, therefore, the individuals with ASD demonstrate a greater perceptual sensitivity to the CS than the control individuals - i.e. they have a greater ability to successfully detect the CS.

While this increased ability was in line with the predictions, it had also been hypothesised that the CS detection of the ASD group would also show some degree of modulation by perceptual load. This was not evident in the results obtained and may be an indication that the highest set size used in the task did not raise perceptual load to a high enough level to exhaust perceptual capacity for the individuals with ASD. This conclusion is consistent with the observation that the error rates of the two groups were equivalent: individuals with ASD were still performing the central task efficiently while detecting the CS.

In Experiment Three within this study, the trials were mixed in an attempt to eliminate any effects of strategy differences between high and low load trials (i.e. each experimental block contained trials of various perceptual load levels). As in the study by MacDonald and Lavie (2008), the effect of perceptual load on CS detection in the typical group was reduced, possibly due to carry-over effects. This resulted in the difference between the ASD and control groups no longer being significant. While this finding may suggest that the two groups are carrying out the task in the 
same way, the potential criticisms of the methodology (see chapter 4) mean that it would be unwise to read too much into the loss of the group difference.

Having used findings from response competition and dual-task paradigms to infer that perceptual capacity in ASD is enhanced, the next step was to find a direct measure of perceptual capacity that could be used to confirm the accuracy of the conclusions that had been drawn. Having examined the literature on visual short term memory (VSTM) and the findings by Riggs et al. (Riggs et al., 2006) regarding the changes in VSTM across childhood, it was proposed that VSTM may be akin to perceptual capacity. In order to evaluate this, Experiment four used a change detection paradigm that is commonly used to assess VSTM capacity (Luck \& Vogel, 1997; Rouder et al., 2008; Vogel et al., 2001). Participants were shown an array of coloured squares $(2,5,8$ or 12$)$ and then indicate whether the colour of a test square (presented subsequently) was the same as that of the corresponding square from the initial array. It was predicted that, if VSTM did indeed reflect perceptual capacity, the individuals with ASD would show enhanced task performance, detecting more of the colour changes - particularly at the larger array sizes.

The results, however, did not appear to show any significant difference between the two groups on either the percentage of changes detected or the VSTM capacity values. Inspection of the data also revealed that the trend appeared to be in the opposite direction - with the ASD group performing slightly worse than the control group. This was surprising not only as it goes against my own prediction, but also because it is not in keeping with the existing data regarding superior changedetection and reduced change-blindness in ASD (Smith \& Milne, 2009). The ASD group also seemed to be showing a higher number of false alarms (responding ‘change' on no-change trials) and also appear to have different response criteria from 
the control group. Though the difference in values was only close to significance ( $p=$ 0.058) the trend indicates that the individuals with ASD have a greater tendency to respond 'change' than the typical adults.

While the results found in this study may indicate that there are no differences in VSTM capacity within ASD, the observation regarding response bias may well shed light on an alternative possible explanation for the absence of group differences in the measures of VSTM calculated within this task. The paradigm used involved a single probe square - rather than displaying the entire array at the test phase. As discussed in chapter 5, this is important in order to keep the amount of decision-making constant across trials of different array sizes and to minimise grouping strategies that may facilitate memory in the larger array sizes. It is possible, however, that the individuals with ASD took the task instructions too literally and, when seeing a single square that they were unsure about, would respond 'change' because, in a sense, every probe display is a change from the initial display (i.e. a number of squares have been replaced by one square).

A recent surprising finding by Franklin and colleagues regarding colour perception in ASD may also have implications for the results (Franklin et al., 2008). In contrast with anecdotal reports and existing experimental data that suggests enhanced sensitivity and attention to colour in autism (Brian et al., 2003; Greenaway \& Plaisted, 2005), Franklin et al.'s study demonstrated that children with ASD were less accurate at colour memory, search and chromatic target-detection than the controls. Given that colour memory and discrimination was a central requisite of the VSTM task used within this thesis, the ASD group may have been disproportionately disadvantaged. 
The large array sizes used may also have masked any potential difference in task performance between the two groups. The maximum array size (12) was a great deal higher than that of previous studies. Based on the prediction that individuals with ASD were likely to outperform the typical adults, this was in order to prevent a ceiling effect - where the individuals with ASD were able to detect all the changes even at the higher set sizes. Having now established that no such superior performance was evident, it may be the case that subtle group differences were lost in the leap between array sizes (two to five, five to eight and eight to twelve).

In order to more meaningfully assess VSTM capacity in ASD, a more appropriate procedure for this clinical group should be employed. One such design may be a change-detection paradigm that requires participants to discriminate between squares based on differing fill patterns (stripes/spots) rather than colour. Arrays of two, three, four and six would be used and the entire sample array would be presented at the test phase. The results from such a study would help further elucidate the magnitude of VSTM capacity in ASD and any relationship between this and perceptual capacity.

Having examined the impact of perceptual load on selective attention to nonsocial letter stimuli, part two of this thesis was devoted to the study of selective attention in the presence of distractor faces. Within mainstream attention research, it has been established that irrelevant face distractors are processed irrespective of perceptual load levels and, as such, they appear to have a 'special status' (Lavie et al., 2003). As discussed in chapter one, social impairments form a core part of the symptom profile of ASD and it was therefore important to examine whether a similar pattern of attention capture by faces is seen for individuals with ASD. Many studies have reported a dysfunction of neural regions of face processing in ASD (Baron- 
Cohen et al., 2000; Dawson et al., 2005) and altered patterns of gaze fixation when looking at faces (Pelphrey et al., 2002). It has been suggested that reduced interest in social stimuli during early childhood, and therefore a lack of looking at faces, may lead to the subsequent face processing and social deficits (Dawson et al., 2005; Grelotti et al., 2002; Schultz, 2005).

In light of such evidence, it was hypothesised that the capacity for processing socially meaningless stimuli would be dissociable from the capacity for processing social stimuli such as faces - i.e. the pattern of greater distractor interference at high set sizes within the ASD group when compared to the typical adults (as was seen in experiments one and two) would not be evident in the task that involved the use of face distractors.

The initial experiment used to examine selective attention and perceptual load in the presence of social distractor stimuli required participants to search for a famous name among a list of non-words and indicate whether it was a pop star or politician. The use of famous faces assumes that all participants have a certain level of world knowledge that will allow them to recognise the identity of the celebrities; however this assumption cannot be made when considering a condition such as ASD. Furthermore, it may have been the case that the distractor faces used in Lavie's task (Lavie et al., 2003) could not be ignored specifically because they were familiar, famous faces. Given that it has been shown that familiarity of a face affects the manner in which it is processed (Rossion et al., 1999; Tong \& Nakayama, 1999), it was important to verify that non-famous faces would also be processed irrespective of perceptual load levels. In order to resolve both these issues, a modified version of Lavie's task was developed. Participants were instructed to classify an anonymous name based on gender while ignoring anonymous male and female distractor faces. 
Results from a group of typical adults (experiment five) demonstrated that the anonymous distractor faces affected task performance even at very high levels of perceptual load; a distractor congruency effect was evident across all the set sizes. This is consistent with Lavie et al's findings regarding attention to famous faces and confirms that the pattern of results they observed was not simply an artefact of the familiar nature of the faces.

Given that the paradigm no longer contained an element of world knowledge, and is therefore suitable for use with a clinical population, it was used to examine selective attention in a group of individuals with ASD. The results from experiment six showed that there was an effect of the irrelevant distractor faces at the lower set sizes but not as the perceptual load of the central task increased. Conversely, as in experiment five, the control individuals showed an effect of distractor type across all the set sizes. It seems that individuals with ASD show the same pattern with face distractors as they did with the non-social letter stimuli (experiments 1-3). However, the task demands of experiments one, two and three (letter stimuli) were very different from those of experiments five and six (face stimuli). In order to more accurately determine whether any 'special' behaviour is seen in response to face distractor stimuli, a task that had identical structure to experiment five and six, but with musical instrument names and pictures was used (experiment seven). By using photographs of instruments and asking participants to classify instrument names into 'wind' or 'string' categories, both tasks involved subordinate category discrimination and a similar level of visual complexity.

Both the ASD and control groups showed a distractor congruency effect at the lower levels of perceptual load that was then eliminated as the perceptual load of the central task increased. By comparing the results from experiments six and seven, 
it could be clearly seen that while the behaviour of the typical adults was markedly different on the two tasks, the individuals with ASD seemed to treat both distractor types in the same way. It appears that for the ASD group, faces do not capture attention in the same way that they do for typical adults. Interestingly, the faces were not completely ignored by individuals with ASD (a congruency effect was seen at the lower set sizes) which suggests that they are not averse to looking at faces entirely. However, the ability to disregard the faces at higher set sizes - something which can be socially detrimental in real-life situations, clearly shows the lack of importance that is placed on such stimuli.

\section{Implications of increased perceptual capacity}

The findings presented within this thesis fit well with the anecdotal and clinical reports of ASD symptomatology, and may well map onto the observed behaviours. Indeed, the prediction concerning increased perceptual capacity originated from an observation of increased distractibility (Burack, 1994; Ciesielski et al., 1990). Though the increased perceptual capacity demonstrated in experiments one, two and three seems to be an ability rather than a disability, and confers an advantage on the tasks within this thesis, in the real world it may well be detrimental. Being able to take in more information at any one time may lead to over arousal in certain situations. Temple Grandin, an author with autism, reports that "when two people are talking at once, it is difficult for me to screen out one voice and listen to the other. My ears are like microphones picking up all sounds with equal intensity. In a noisy place I can't understand speech, because I can't screen out the background noise" (Grandin, 1995). This over-arousal has previously been reported in ASD (Hermelin \& O'Connor, 1970; e.g. Hutt et al., 1964; Hutt et al., 1965; Raymaekers et 
al., 2004). Many of those reporting over-arousal in ASD attributed it to an inability to effectively filter out irrelevant information. The work within this thesis suggests that it may instead be due to the increased perceptual capacity, which results in far more visual information being processed simultaneously before the capacity is saturated.

This finding has important practical implications for individuals with ASD. Forster and Lavie (2007) showed that individual differences in perceptual load effects are correlated with day-to-day distractibility levels. Individuals who scored highly on the Cognitive Failures Questionnaire (CFQ, Broadbent et al., 1982) showed greater distractor interference effects at the low set sizes than those who obtained a low score on the CFQ. Interestingly, Forster and Lavie showed that at the high levels of perceptual load the distraction from irrelevant stimuli was eliminated for both the high and low scoring CFQ groups. They conclude that high levels of perceptual load make everyone equal and suggest that increasing perceptual load may be a useful tool in the quest to reduce the level of distractibility in everyday tasks such as driving and computer work. In the experiment presented within this chapter, the highest set size did not equate the groups, but it is possible that introducing a higher level of perceptual load would lead to elimination of CS processing in both groups. Finding this level, above which early selection is exhibited in both groups, would be clinically beneficial. Though the increased perceptual capacity seems to offer an advantage in the dual-task paradigm, it is clear that in real-world situations processing a great deal of visual stimuli simultaneously could be overwhelming and debilitating. Perceptual load levels may be manipulated in order to help individuals with ASD perform more effectively on tasks where distractibility is a hindering factor. 
Increased perceptual capacity may also go some way to explain savant abilities in ASD: where individuals seem to be able to process an extremely large amount of information at any one time. For example, the artist Stephen Wiltshire can look at a complex visual scene and then draw it from memory - with an extremely high level of detail (i.e. the correct number of windows in each building, the correct layout of buildings etc). It is definitely tempting to conclude that such artists have an increased perceptual capacity. Indeed in a future study it would be interesting to test an individual with savant abilities on a task of selective attention and perceptual load. It is important to mention that neither savant abilities, nor reports of over-arousal, seem restricted to the visual domain. There is evidence of auditory hyperacuity in ASD (Ney, 1979; Talay-Ongan \& Wood, 2000) which may indicate over arousal in the auditory domain and there are also a number of musical savants with ASD. This raises the question: to what extent can the visual abnormalities that have been found within this thesis be generalised to the auditory domain? While hyperacuity could be related to the frequency of the sound, it may also be due to an increased amount of auditory information being processed, or an inability to ignore distracting sounds. It would be interesting, therefore, to explore selective attention and perceptual load in the auditory domain. A set of experiments have been carried out on selective attention in the auditory domain within ASD (Hismjatullina, 2006). On a dichotic listening task, she found that while the overall response times were equivalent between the two groups, the ASD group made more errors- i.e. they were distracted by, and processed, the unattended stream to a greater extent. The authors mention that they are unsure as to how the reaction times can be unaffected while the error rates are higher in the ASD group. The idea of increased perceptual capacity would offer a plausible explanation for this finding. Hismjatullina also looked at social 
aspects of auditory selective attention by examining whether individuals with ASD would orient as much to their own name as typical adults. She found that, as with the typical group, the ASD individuals were distracted by their own names and personal items. This was taken as evidence for intact covert social orienting, but it is possible that this was only seen due to the low perceptual load of the task as Hismjatullina mentions that her task is similar to the 'easy-condition' of Teder-Salejarvi et al. (Teder-Salejarvi et al., 2005). It would be meaningful to take this research one step further by introducing the issue of perceptual load. In the dichotic listening task, conditions with different levels of load should be created to examine the effect on task performance of both groups. On the social task, it would be predicted that one could raise the perceptual load of the task to levels where the individuals with ASD are ignoring their own name but the typical individuals are not.

Aside from the relevance to everyday behaviour seen in ASD, how do the findings from the experiments in this thesis fit with current theories of cognition in ASD such as weak central coherence (WCC), enhanced perceptual functioning (EPF) and visual search behaviour? As discussed in chapter 1, the theory of WCC suggests that individuals with ASD either have a deficit in extracting the overall meaning of a stimulus or visual scene, or have a cognitive style that tends towards processing the local, rather than global, aspects of stimuli (Frith, 1989; Happe, 1999). The superior performance on tasks such as the Embedded Figures Task and IQ subtests involving block design and pattern construction has been used as evidence for WCC. Following the demonstration that global processing is not always altered in ASD (e.g. Plaisted et al., 1999), the EPF model was put forward as an alternative to WCC (Mottron \& Burack, 2001). This framework suggests an enhanced perceptual flow of information in ASD, leading to positive symptoms such as enhanced memory for visual patterns 
and negative impacts such as a difficulty in controlling perceptual processes (Mottron et al., 2006). The observations upon which these two theories are based may, however, also be explained by enhanced perceptual capacity. An increased capacity would allow an individual to take in more information from the visual field at any given time. While this cannot explain why individuals with ASD may prefer to process the local aspects of stimuli, it suggests that such individuals are able to process additional details that typical individuals ignore due to capacity limits. In addition, if an individual can process a greater number of elements within a visual search task, then they will be able to detect the target faster than those who can only detect a small number of items simultaneously. Increased perceptual capacity would also offer an explanation for the reduced change-blindness that has been reported in ASD (Smith \& Milne, 2009). The authors suggest that the enhanced sensitivity to change that was seen in their ASD group, when compared to a control group, was due to a greater number of items entering visual awareness. Though they then attribute this to a deficit in attentional gating, it may be that increased perceptual capacity is responsible. Furthermore, the conflicting reports regarding unaltered change-detection abilities (Burack et al., 2009b; Fletcher-Watson et al., 2006) may be explained within the perceptual load framework. These two studies used paradigms that involved participants detecting changes within pairs of pictures, whereas Smith and Milne required participants to detect continuity errors in film clips. The latter is arguably a more difficult task with a higher level of perceptual load. It is therefore in keeping with the findings of this thesis that a difference between the abilities of individuals with ASD and control individuals was only evident under this higher level of perceptual load 
While the findings of this thesis do not indicate whether increased perceptual capacity causes the observed behaviours mentioned above, it is clearly a concept that may well be related and should be further explored.

While these models of WCC and EPF suggest that greater perceptual capacity may be linked to enhanced attention to detail, it is also possible that a lack of topdown control of attention is responsible for the pattern of results seen in part one of this thesis. Attention can be split into top-down and bottom-up processing. Top-down attention refers to a situation where higher-cognitive processes such as working memory, context and expectation influence the attentional set and therefore affect what stimuli are attended to. For example, if you are searching for a flower then the flowers in the visual field will be attended to in preference to other available stimuli. Conversely, bottom-up processing is driven by the salience of the stimuli. For example, if a flower in a field is very distinctive then it will draw ones attention to it, regardless of whether it was important to the current situation. Lavie and colleagues have extended load theory to include the impact of working memory load and cognitive control on selective attention. They suggest that in order to direct attention appropriately, individuals must maintain stimulus priorities in working memory - i.e. to remember which stimuli are relevant. Therefore, loading working memory processes should result in reduced differentiation between high- and low-priority stimuli (targets and distractors) and lead to the opposite effect from that of perceptual load; as working memory load increases, the extent of distractor processing will also increase (Lavie, 2000; Lavie et al., 2004b). This was investigated by presenting participants with a selective attention task that was preceded by a memory set (a string of digits) and followed by a memory probe digit (participants must indicate whether the digit was part of the memory set). The working memory of the task was 
manipulated by altering the number of digits in the memory set. The results demonstrated that, in line with their original prediction, the influence of distractors in the selective attention task was greater under conditions of high working memory load (Lavie et al., 2004a). It could therefore be suggested that the increased effect of distractors, seen in experiments one and two, was a result of the working memory load of the task disproportionately affecting the individuals with ASD, resulting in a lack of the top-down control that is necessary to prevent distractor interference. If this was the case, however, then you would expect to see an effect of increased working memory load across all set sizes and conditions (given that the task instructions remain constant for the entire experiment). This would be manifest as an increased distractor effect at all levels of perceptual load. Furthermore, it can be seen from the results of Lavie's experiments that the loading of working memory also detrimentally affected task performance. As mentioned above, the distractor effect (i.e. the difference between the compatible and incompatible trials) in Lavie et al. (2004a) was greater for the high working memory load condition. The response times to both the compatible and incompatible trials were also both slower for the high working memory load condition. If the pattern of results in my experiments was due to high working memory load demands on the individuals with ASD then you would expect to see a general difference in the response times between the two groups something which is not seen. Though it seems that the idea of an attentional-control deficit does not underlie the results observed within this thesis, it will be important in future research to explore the issue of working memory and perceptual load in ASD. Since ASD is known to involve impaired executive functioning (Ozonoff et al., 1991) which may include a deficit in working memory (Bennetto et al., 1996; Russell et al., 1996), it is hypothesised that lower levels of working memory load would be 
sufficient to result in increased distractor processing in ASD. I propose to investigate this predicted dissociation between superior performance on selective attention tasks with high perceptual load and inferior performance with high working memory load in a subsequent study.

This proposed reduction in working memory capacity may also offer an alternative explanation for the differing reports of change-blindness in ASD. In Smith and Milne's task (2009), participants were asked to watch a video clip of a chef baking buns, and were then questioned about the clip: both regarding any continuity errors that were observed, but also regarding the scene itself (e.g. how many eggs were used). This places a much higher demand on working memory (the details of the clip must be retained while searching for errors) than the picture comparison tasks used in the other two studies of change detection in ASD (Burack et al., 2009b; Fletcher-Watson et al., 2006). The higher working memory load may make the individuals with ASD more susceptible to distraction from the details within the scene which, on this task, facilitates detection of the continuity errors and results in superior task performance.

Finally, before moving on to discuss the implications of the experiments with social distractors, I will explore whether the concept of increased perceptual capacity, and resulting pattern of increased distractibility at high levels of load, can account for the varying attentional abnormalities in ASD. The findings regarding increased perceptual capacity and the perceptual load model would predict that cases where individuals with ASD were seen to show a greater influence of distractors (previously taken as evidence of poor filtering ability) would involve tasks with a moderately high perceptual load - such that only the individuals with ASD would have spare processing capacity left over for distractor processing. 
Conversely, it would be predicted that the studies where no difference between groups was detected would involve either very high or very low levels of load. At very high levels of load, the central task would exhaust the perceptual capacity of both groups; therefore eliminating distractor interference, while at very low levels of load (likely to be indexed by ceiling effects in both groups) all individuals would show an effect of distractors.

The study by Burack (1994) is an example of a study that reports underselectivity and filtering deficits i.e. increased distractibility in ASD. His task involved target detection $(\mathrm{O}$ or + ) in the presence of two or four non-target shapes (\# $* \Delta$ or $\left.{ }^{\wedge}\right)$. The target was presented with either zero, two or four distractors - and these distractors were either $2^{\circ}$ visual angles (close condition) or $6^{\circ}$ visual angles (far condition) away from the target. This would be around the level of perceptual load for which we would expect ASD individuals to show a greater interference effect. In line with this, the results indicated that the individuals with ASD do show a greater interference effect of the distractor shapes but do not show increased error rates. Ciesielski et al.(1990) also suggest filtering deficits in ASD. Participants were presented with stimuli that had both a visual and auditory component and were told to selectively attend to only one domain. Within each domain there were rare events embedded among a stream of more frequent events (a high tone among low tones and a green flash among red flashes). Participants were told which modality they should attend to and were required to indicate, with a key-press, when they observed the rare event. Though the paradigm is not entirely analogous to those used within this thesis, the results from the visual domain may be relevant. Though the sensitivity to the rare target (d'), response criteria $(\beta)$ and percentage of correct target detections were not significantly different from those of the control group, the individuals with 
ASD responded more often to the rare events in the unattended modality (a striking increase from $4.5 \%$ in controls to $32 \%$ in the ASD group). This finding is very much in line with the idea of increased perceptual capacity in ASD.

In contrast, Iarocci \& Burack (2004) found no difference between the behaviour of children with ASD and typical children on a detection task in the presence and absence of distractors. In this paradigm, the perceptual load of the task seemed to be very low; i.e. in the distractor-present condition the target was flanked by two unrelated distractor symbols such that the three symbols were in a line with the target always presented in the middle. The task performance was at ceiling for both the ASD and control groups (error rates of $0.5 \%$ and $0.8 \%$ respectively). At this level of load, one would expect that the perceptual capacity of the typical participants would not have been filled and therefore both groups would show equivalent distractor interference effects. This was indeed the case: the difference in RTs between the two distractor conditions was investigated and the authors found that both groups showed equivalent increase of RT in the distractor-present condition.

When it comes to looking at reports of over-selectivity in ASD within the context of perceptual load, the issue is slightly more complex. Load theory and the idea of increased perceptual capacity in ASD would not predict over-selectivity at any level of load. Within this thesis, however, there was evidence of over-selectivity with respect to social stimuli and it seems that many of the studies suggesting overselectivity in ASD involve a social component. Moreover, the anecdotal accounts of over-focussed behaviour in ASD most commonly refer to situations where individuals have fixated on a random aspect of the environment to such an extent that there is no response to social attention bids such as own name calling. 
The early studies of overly focussed attention (Schriebman \& Lovaas, 1973)

involved social cues. More recently Garretson, Fein and Waterhouse (1990) only found differences in selectivity between the groups in the social reward condition, and Pierce et al.(Pierce et al., 1997) presented evidence of over-selectivity in ASD but used a task that involved one to four social cues.

The other studies concerning over-selectivity in ASD that were mentioned in chapters two and three did not contain a social aspect, but they also did not involve the blocking out of distractor stimuli. Such studies (Lovaas et al., 1971; Lovaas et al., 1979; Rincover \& Ducharme, 1987) drew findings based on the behaviour in response to multi-componential stimuli and a failure to integrate these components. It seems that tasks of this type have different demands and results may reflect different abilities from those tested on more classic filtering tasks.

While not all studies fit the pattern, there does seem to be an interesting trend whereby many reports of over selectivity involve social stimuli, those reporting no difference between control and ASD groups involve tasks with low load and those that demonstrate reduced filtering use high load paradigms.

\section{Implications of reduced interference by social distractors}

There are a number of implications of the finding that individuals with ASD do not appear to assign special salience to face distractors and are therefore able to ignore them under high levels of perceptual load. The tasks used within this thesis offer an alternative to experimental paradigms which require participants to actively attend to faces. While results from the latter have given rise to evidence for a variety of impairments in face processing, the set-up used here in experiments four and five creates a situation that exposes whether faces naturally capture attention in ASD - 
even when participants are explicitly instructed to ignore them. As mentioned above, it was clear from the results that only the typical group processed these social stimuli in a mandatory and automatic fashion.

This finding may help to elucidate the reasons underlying the abnormal social interaction and altered face processing abilities seen in the condition. Studies of early behaviours report that, when compared to typically developing children, children with ASD spend less time looking at faces (see Riby \& Hancock, 2008). The absence of special salience to faces and evidence that faces do not have preferential access to attention in ASD (as observed in experiment five) may be the cause of these altered early behaviours. Alternatively, the reduced salience may be a consequence of the lack of experience processing faces at a young age. Certainly, a lack of attention to faces would have serious consequences for social communicative development. As discussed in chapter 6 , the subsequent specialization of the face-processing network may well rely on adequate social input during the critical period of early infancy. To investigate this possible causal relationship, it is important to now devise studies which can assess attention with respect to social stimuli in children with ASD; allowing the developmental trajectories of any altered attentional behaviour to be explored. Such tasks could employ eye-tracking procedures which, though not equivalent to attention, may well map on to similar processes. This would render them accessible to a much younger population by removing task demands.

The findings from this research on social attention may also have practical application for the treatment of individuals with ASD. The results suggest the importance of interventions which simply promote looking/orienting to faces. This would be particularly important early in development - with the logic that, given the 
relevant input, the face-processing system would then become specialised without further intervention.

\section{Further study}

There are a number of potential studies that have been mentioned in this thesis which should be performed in order to allow the field to move forwards. Firstly, following on from the idea of increased perceptual capacity in ASD, the issue of selective attention and working memory load should be explored. Working memory is used to maintain current stimulus processing priorities thus minimizing the processing of irrelevant stimuli. Therefore, in contrast with perceptual load, high working memory load increases distractor processing. Since ASD is known to involve impaired executive functioning it is hypothesised that lower levels of working memory load would be sufficient to result in increased distractor processing. This predicted dissociation between superior performance on selective attention tasks with high perceptual load and inferior performance with high working memory load should be investigated by carrying out a selective attention task under various levels of working memory load.

Secondly, a more appropriate test of VSTM should be employed to directly assess the proposed increased perceptual capacity in ASD. Rather than using coloured squares, a version that used a set of lines - defined by orientation - would overcome the potential issue of abnormal colour processing. Using array sizes of two, three, four and six (rather than two, five, eight and 12) would also allow more detailed information regarding the capacity of each group to be ascertained.

Thirdly, it would be interesting to explore the issue of selective attention and perceptual load in the auditory domain. Dichotic listening tasks with varying levels 
of load could begin to investigate whether an enhanced perceptual capacity is specific to the visual domain or extends across modalities.

Fourthly, neuroimaging techniques should be used to advance the findings of this thesis. Signals in the inferior parietal sulcus (implicated in selective attention) could be used to gain further insight into the nature of perceptual capacity in ASD, while FFA activation could be used to more conclusively establish to what extent the face distractors are processed on tasks of selective attention and perceptual load.

Lastly, it should be highlighted that all the experiments in this thesis were performed with an adult population. It is currently not known whether the same pattern of increased distractor processing at high levels of perceptual load, and reduced attention to faces is seen within childhood or whether this develops later in life. It is therefore important to also consider this issue developmentally.

\section{Conclusion}

The work presented here has, for the first time, used the Load Theory of Attention and Cognitive Control to investigate selective attention in ASD. The findings offer preliminary evidence for increased perceptual capacity in ASD - with respect to both unconscious and conscious processes. This has implications for our understanding of some of the non-social abnormalities in ASD such as savant-like abilities (in some individuals), and the ability to notice seemingly irrelevant details in the environment. The thesis also presents new evidence that attention to faces differs in ASD. Most previous work on face processing in ASD has highlighted differences in recognition of emotion and/or feature processing of faces; the work in this thesis shows that regardless of face processing skills, faces are less salient for individuals with ASD and therefore are not assigned the high priority that is seen in typical 
adults. This second part of the thesis brings together findings on selective attention with work on social processing, in an attempt to find basic abnormalities which might be fundamental in explaining the disorder.

While the majority of findings regarding ASD focus on deficits within the condition, the research presented here suggests that in some circumstances these altered behaviours can be usefully employed to reduce distraction while focusing attention on tasks (experiment five) and increase detection of necessary details within a visual scene (experiment two). The findings of this work could also potentially provide individuals with ASD an important insight, which would enable them to maximise their potential by exploiting these strengths and minimising the impact of any weaknesses, as well as explaining their condition to others. Finally, it is hoped that a greater understanding of what characterizes ASD individuals' unusual attentional and perceptual profile may ultimately help in the design of educational and therapeutic programs, which capitalize on their unique cognitive profile. 


\section{Appendix I}

Participant involvement across all studies - ASD group

\begin{tabular}{|c|c|c|c|c|c|c|c|}
\hline Participant ID & $\operatorname{Exp} 1$ & $\operatorname{Exp} 2$ & $\operatorname{Exp} 3$ & $\operatorname{Exp} 4$ & Exp5 & Exp6 & Exp7 \\
\hline 51 & $\checkmark$ & $\checkmark$ & $\checkmark$ & $\checkmark$ & & $\checkmark$ & $\checkmark$ \\
\hline 52 & $\checkmark$ & $\checkmark$ & $\checkmark$ & $\checkmark$ & & $\checkmark$ & $\checkmark$ \\
\hline 53 & $\checkmark$ & $\checkmark$ & $\checkmark$ & $\checkmark$ & & $\checkmark$ & $\checkmark$ \\
\hline 54 & $\checkmark$ & & & & & & \\
\hline 55 & $\checkmark$ & $\checkmark$ & $\checkmark$ & $\checkmark$ & & $\checkmark$ & $\checkmark$ \\
\hline 56 & $\checkmark$ & & & & & $\checkmark$ & \\
\hline 57 & $\checkmark$ & $\checkmark$ & $\checkmark$ & $\checkmark$ & & $\checkmark$ & $\checkmark$ \\
\hline 58 & $\checkmark$ & $\checkmark$ & $\checkmark$ & $\checkmark$ & & $\checkmark$ & $\checkmark$ \\
\hline 59 & $\checkmark$ & $\checkmark$ & $\checkmark$ & $\checkmark$ & & $\checkmark$ & $\checkmark$ \\
\hline 60 & $\checkmark$ & & & & & $\checkmark$ & \\
\hline 61 & $\checkmark$ & & & & & $\checkmark$ & \\
\hline 62 & $\checkmark$ & $\checkmark$ & $\checkmark$ & $\checkmark$ & & $\checkmark$ & $\checkmark$ \\
\hline 63 & $\checkmark$ & $\checkmark$ & $\checkmark$ & $\checkmark$ & & $\checkmark$ & $\checkmark$ \\
\hline 64 & $\checkmark$ & & & & & & \\
\hline 65 & $\checkmark$ & & & & & $\checkmark$ & \\
\hline 66 & $\checkmark$ & & & & & & \\
\hline 67 & $\checkmark$ & & & & & $\checkmark$ & \\
\hline 68 & $\checkmark$ & $\checkmark$ & $\checkmark$ & $\checkmark$ & & $\checkmark$ & $\checkmark$ \\
\hline 69 & $\checkmark$ & $\checkmark$ & $\checkmark$ & $\checkmark$ & & $\checkmark$ & $\checkmark$ \\
\hline 70 & & $\checkmark$ & $\checkmark$ & $\checkmark$ & & & $\checkmark$ \\
\hline \multicolumn{8}{|l|}{71} \\
\hline 72 & & $\checkmark$ & $\checkmark$ & $\checkmark$ & & & $\checkmark$ \\
\hline 73 & & $\checkmark$ & $\checkmark$ & $\checkmark$ & & & $\checkmark$ \\
\hline 74 & & $\checkmark$ & $\checkmark$ & $\checkmark$ & & & $\checkmark$ \\
\hline 75 & & $\checkmark$ & $\checkmark$ & $\checkmark$ & & & $\checkmark$ \\
\hline 76 & & $\checkmark$ & $\checkmark$ & $\checkmark$ & & & $\checkmark$ \\
\hline
\end{tabular}


Participant involvement across all studies - control group

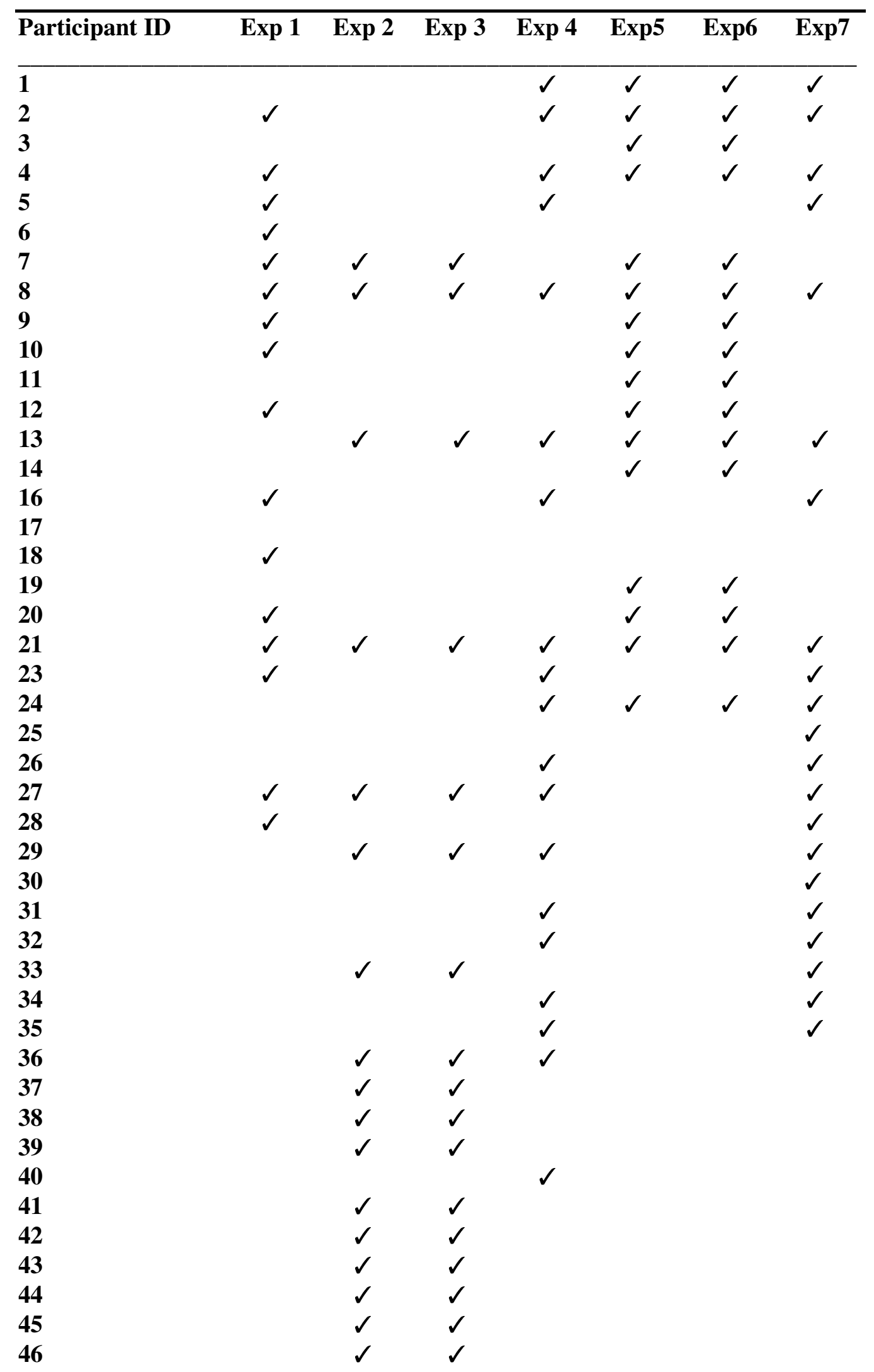




\section{Appendix II}

Materials used for experiments five and six: selective attention and perceptual load in the presence of face distractor stimuli

Face Distractors:
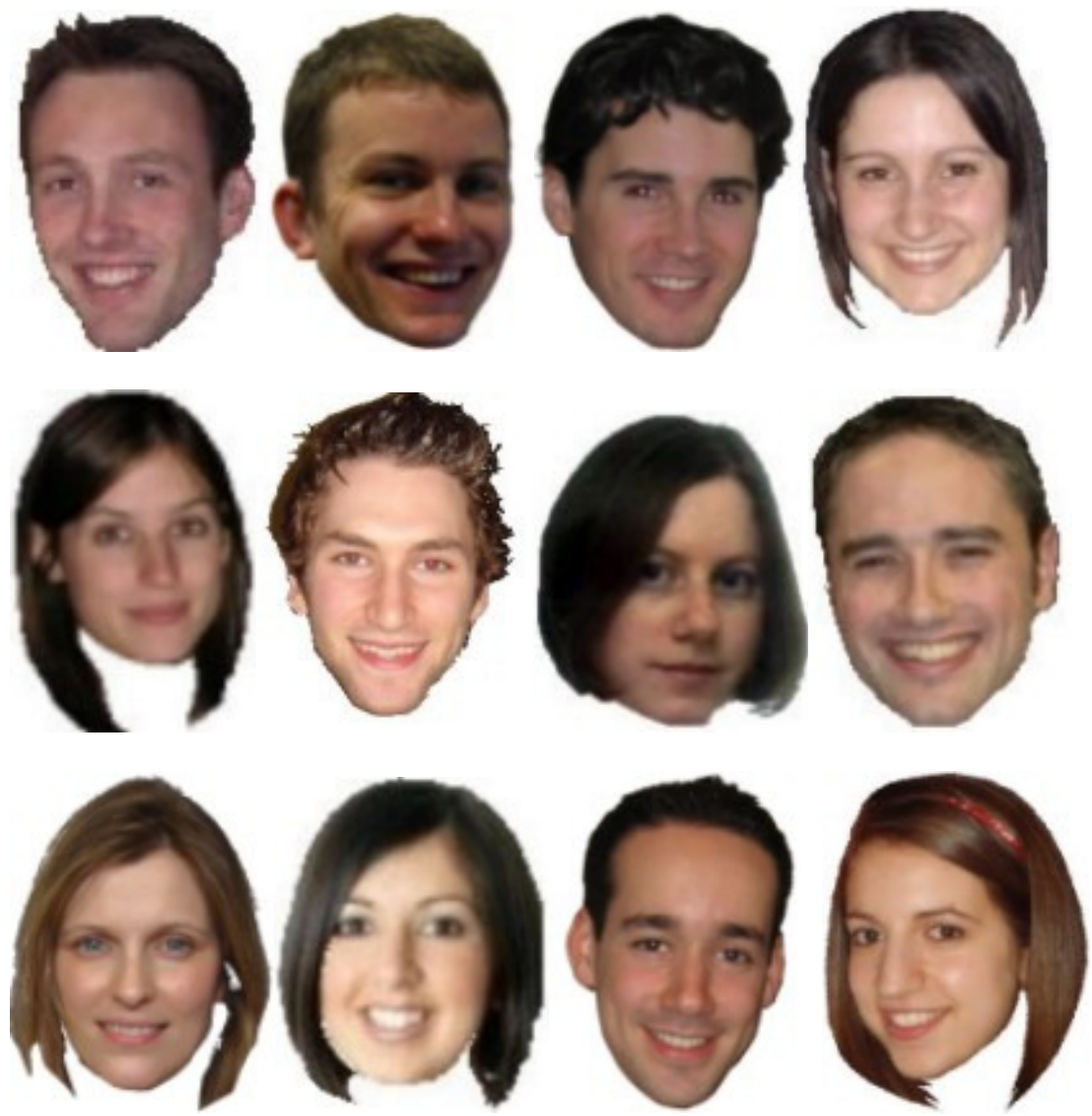

Names used:

Non-words:

Male Female

$\begin{array}{llll}\text { John } & \text { Lucy } & \text { Gydhs } & \text { Zlhdp } \\ \text { James } & \text { Mary } & \text { Sxfdret } & \text { Pgcvlsr } \\ \text { David } & \text { Sarah } & \text { Blkp } & \text { Jfpgxw } \\ \text { Henry } & \text { Jane } & \text { Dmhso } & \text { Blwcy } \\ \text { Thomas } & \text { Katie } & \text { Ifgbter } & \text { Mjgtpf } \\ \text { Michael } & \text { Sophie } & \text { Kpsigk } & \text { Idnhl }\end{array}$




\section{Appendix III}

Materials used for experiment seven: selective attention and perceptual load in the presence of musical instrument distractor stimuli

Face Distractors:
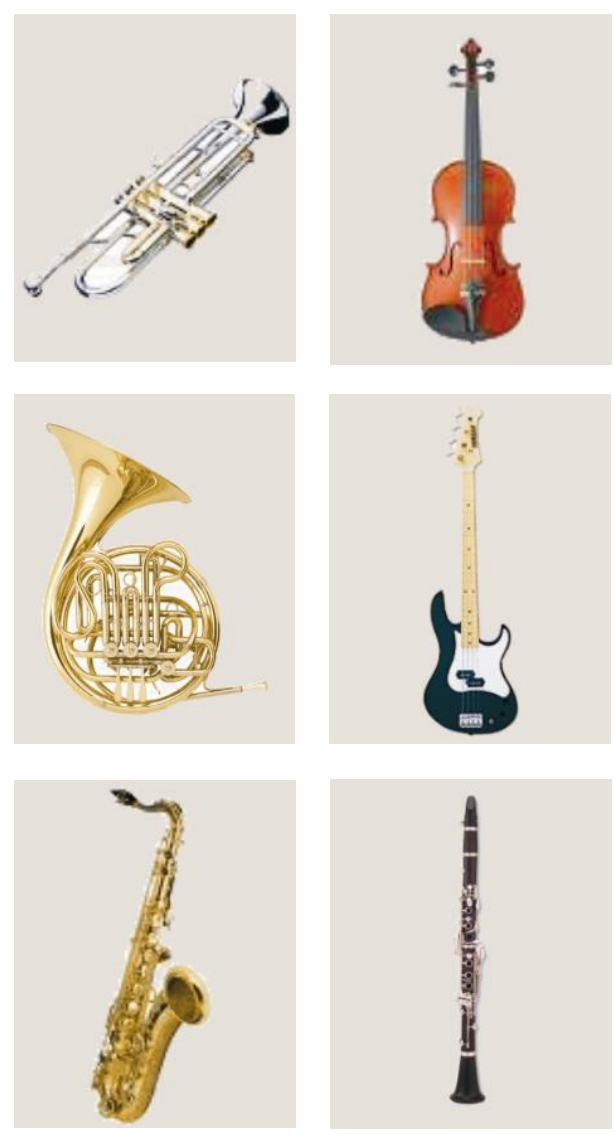

Names used:

$\begin{array}{ll}\text { String } & \text { Wind } \\ \text { Cello } & \text { Trumpet } \\ \text { Violin } & \text { Clarinet } \\ \text { Bass } & \text { Trombone } \\ \text { Harp } & \text { Tuba } \\ \text { Guitar } & \text { Saxophone } \\ \text { Banjo } & \text { Horn }\end{array}$
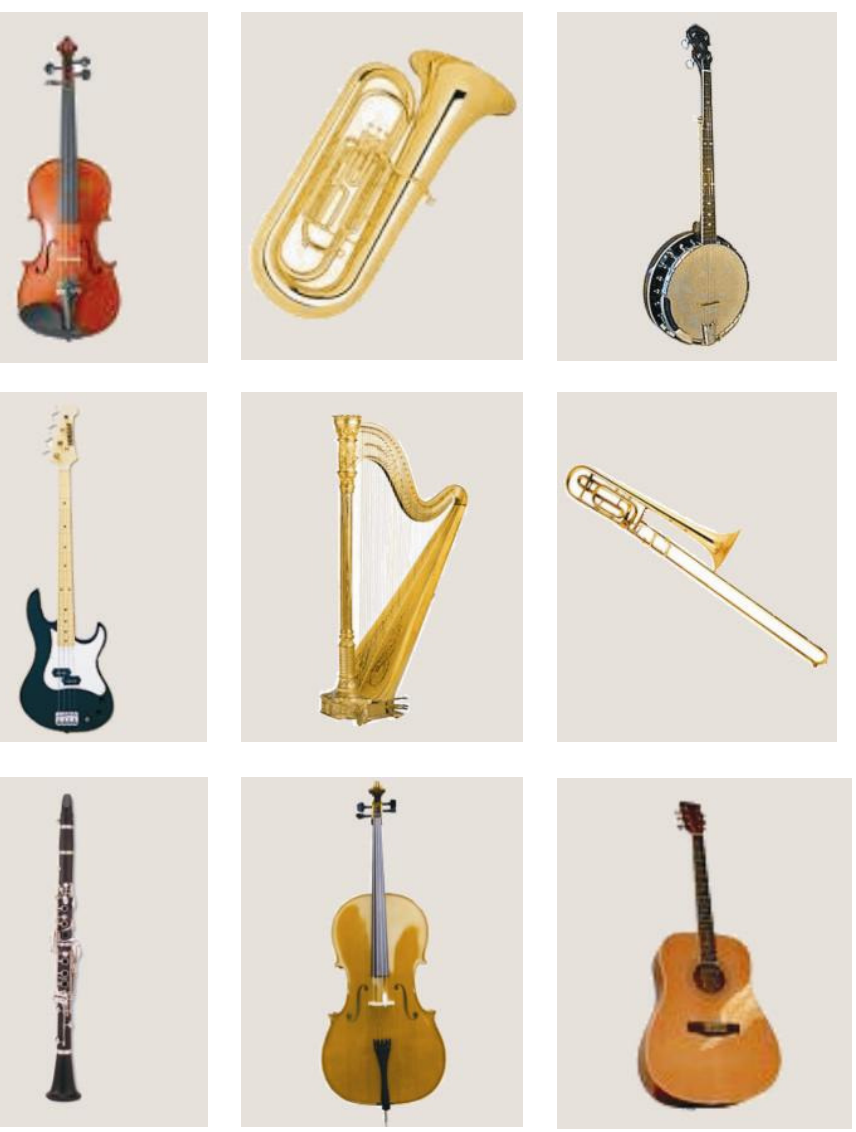

Non-words:

$\begin{array}{ll}\text { Gydhs } & \text { Zlhdp } \\ \text { Sxfdret } & \text { Pgcvlsr } \\ \text { Blkp } & \text { Jfpgxw } \\ \text { Dmhso } & \text { Blwcy } \\ \text { Ifgbter } & \text { Mjgtpf } \\ \text { Kpsigk } & \text { Idnhl }\end{array}$




\section{References}

Abell, F., Happe, F., \& Frith, U. (2000). Do triangles play tricks? Attribution of mental states to animated shapes in normal and abnormal development. Journal of Cognitive Development, 15, 1-20.

Adolphs, R., Sears, L., \& Piven, J. (2001). Abnormal processing of social information from faces in autism. J.Cogn Neurosci., 13, 232-240.

Allen, G. \& Courchesne, E. (2001). Attention Function and Dysfunction in Autism. Frontiers in Bioscience, 6, 105-119.

Allison, T., Ginter, H., McCarthy, G., Nobre, A. C., Puce, A., Luby, M. et al. (1994). Face recognition in human extrastriate cortex. J.Neurophysiol., 71, 821-825.

Allison, T., McCarthy, G., Belger, A., Puce, A., Luby, M., Kim, J. et al. (1995). What is a face? Electrophysiological responsiveness of human extrastriate visual cortex to human and animal faces, face components, and complex objects. Proc.Cognit.Neurosci.Soc. 2, 49.

Ref Type: Abstract

Allison, T., Puce, A., \& McCarthy, G. (2000). Social perception from visual cues: role of the STS region. Trends Cogn Sci., 4, 267-278.

Aman, C. J., Roberts, R. J., Jr., \& Pennington, B. F. (1998). A neuropsychological examination of the underlying deficit in attention deficit hyperactivity disorder: frontal lobe versus right parietal lobe theories. Dev.Psychol., 34, 956-969.

American Psychiatric Association (1994). DSM-IV Diagnostic and Statistical Manual of Mental Disorders. (4th ed.) Washington DC: American Psychiatric Association.

Anderson, L. T., Campbell, M., Grega, D. M., Perry, R., Small, A. M., \& Green, W. H. (1984). Haloperidol in the treatment of infantile autism: effects on learning and behavioral symptoms. Am.J.Psychiatry, 141, 1195-1202.

Asperger, H. (1944). Die "Autistischen Psychopathen" im Kindesalter. Archiv fur Psychiatrie und Nervenkrankheiten, 117, 76-136.

Astington, J. \& Baird, J. (2005). Why language matters for theory of mind. Oxford, UK: Oxford University Press.

Atkinson, R. C. \& Shiffrin, R. M. (1968). Human memory: A proposed system and its control processes. In K.W.Spence \& J. T. Spence (Eds.), The Psychology of Learning and Motivation: Advances in Research and Theory (Vol. 2) (pp. 89-195). Academic Press.

Atkinson, R. C. \& Shiffrin, R. M. (1971). The control of short-term memory. Sci.Am., 225, 82-90. 
Attwood, A., Frith, U., \& Hermelin, B. (1988). The understanding and use of interpersonal gestures by autistic and Down's syndrome children. J.Autism Dev.Disord., 18, 241-257.

Auyeung, B., Baron-Cohen, S., Ashwin, E., Knickmeyer, R., Taylor, K., \& Hackett, G. (2008). Fetal testosterone and autistic traits. Br.J.Psychol., 100, 1-22.

Axelrod, B. N., Goldman, R. S., Tompkins, L. M., \& Jiron, C. C. (1994). Poor differential performance on the Wisconsin Card Sorting Test in schizophrenia, mood disorder, and traumatic brain injury. Neuropsychiatry, Neuropsychology and Behavioural Neurology, 7, 20-24.

Baddeley, A. D. \& Hitch, G. J. L. (1974). Working memory. In G.A.Bower (Ed.), The psychology of learning and motivation: advances in research and theory (pp. 47-89). New York: Academic Press.

Bailey, A., Le, C. A., Gottesman, I., Bolton, P., Simonoff, E., Yuzda, E. et al. (1995). Autism as a strongly genetic disorder: evidence from a British twin study. Psychol.Med., 25, 63-77.

Bailey, A. J., Braeutigam, S., Jousmaki, V., \& Swithenby, S. J. (2005). Abnormal activation of face processing systems at early and intermediate latency in individuals with autism spectrum disorder: a magnetoencephalographic study. Eur.J.Neurosci., 21, 2575-2585.

Baird, G., Simonoff, E., Pickles, A., Chandler, S., Loucas, T., Meldrum, D. et al. (2006). Prevalence of disorders of the autism spectrum in a population cohort of children in South Thames: the Special Needs and Autism Project (SNAP). Lancet, $368,210-215$.

Banks, M. S. \& Salapatek, P. (1981). Infant pattern vision: a new approach based on the contrast sensitivity function. J.Exp.Child Psychol., 31, 1-45.

Baranek, G. T. (1999). Autism during infancy: a retrospective video analysis of sensory-motor and social behaviors at 9-12 months of age. J.Autism Dev.Disord., 29, 213-224.

Baron-Cohen, S. (1989). The autistic child's theory of mind: a case of specific developmental delay. J.Child Psychol.Psychiatry, 30, 285-297.

Baron-Cohen, S. (1990). Autism: a specific cognitive disorder of "mindblindness". Int.Rev.Psychiatry, 2, 79-88.

Baron-Cohen, S. (2002). The extreme male brain theory of autism. Trends Cogn Sci., 6, 248-254.

Baron-Cohen, S. (2006). The hyper-systemizing, assortative mating theory of autism. Prog.Neuropsychopharmacol.Biol.Psychiatry, 30, 865-872.

Baron-Cohen, S. \& Hammer, J. (1997). Is autism an extreme form of the male brain? Advances in Infancy Research, 11, 193-217. 
Baron-Cohen, S., Jolliffe, T., Mortimore, C., \& Robertson, M. (1997). Another advanced test of theory of mind: evidence from very high functioning adults with autism or asperger syndrome. J.Child Psychol.Psychiatry, 38, 813-822.

Baron-Cohen, S., Leslie, A. M., \& Frith, U. (1985). Does the autistic child have a "theory of mind"? Cognition, 21, 37-46.

Baron-Cohen, S., Lutchmaya, S., \& Knickmeyer, R. (2004). Prenatal testosterone in mind: amniotic fluid studies. Cambridge, MA: MIT Press.

Baron-Cohen, S., Ring, H., Moriarty, J., Schmitz, B., Costa, D., \& Ell, P. (1994). Recognition of mental state terms. Clinical findings in children with autism and a functional neuroimaging study of normal adults. Br.J.Psychiatry, 165, 640649.

Baron-Cohen, S., Ring, H. A., Bullmore, E. T., Wheelwright, S., Ashwin, C., $\&$ Williams, S. C. (2000). The amygdala theory of autism. Neurosci.Biobehav.Rev., $24,355-364$.

Baron-Cohen, S., Spitz, A., \& Cross, P. (1993). Can children with autism recognize surprise? Cognition and Emotion, 7, 507-516.

Baron-Cohen, S., Wheelwright, S., Hill, J., Raste, Y., \& Plumb, I. (2001). The "Reading the Mind in the Eyes" Test revised version: a study with normal adults, and adults with Asperger syndrome or high-functioning autism. J.Child Psychol.Psychiatry, 42, 241-251.

Bauman, M. L. (1996). Brief report: neuroanatomic observations of the brain in pervasive developmental disorders. J.Autism Dev.Disord., 26, 199-203.

Beech, A., Powell, T., McWilliam, J., \& Claridge, G. (1989). Evidence of reduced 'cognitive inhibition' in schizophrenia. Br.J.Clin.Psychol., 28 ( Pt 2), 109116.

Belmonte, M. K. (2000). Abnormal attention in autism shown by steady-state visual evoked potentials. Autism, 4, 269-285.

Belmonte, M. K., Cook, E. H., Jr., Anderson, G. M., Rubenstein, J. L., Greenough, W. T., Beckel-Mitchener, A. et al. (2004). Autism as a disorder of neural information processing: directions for research and targets for therapy. Mol.Psychiatry, 9, 646-663.

Bennetto, L., Pennington, B. F., \& Rogers, S. J. (1996). Intact and impaired memory functions in autism. Child Dev., 67, 1816-1835.

Bentin, S., Allison, T., Perez, E., Puce, A., \& McCarthy, G. (1996). Electrophysiological studies of face perception in humans. J.Cogn Neurosci., 8, 551565 .

Benton, A. L. (1980). The neuropsychology of facial recognition. Am.Psychol., 35, 176-186. 
Berger, H. J., van Spaendonck, K. P., Horstink, M. W., Buytenhuijs, E. L., Lammers, P. W., \& Cools, A. R. (1993). Cognitive shifting as a predictor of progress in social understanding in high-functioning adolescents with autism: a prospective study. J.Autism Dev.Disord., 23, 341-359.

Birch, S. A. \& Bloom, P. (2003). Children are cursed: an asymmetric bias in mental-state attribution. Psychol.Sci., 14, 283-286.

Bird, G., Catmur, C., Silani, G., Frith, C., \& Frith, U. (2006). Attention does not modulate neural responses to social stimuli in autism spectrum disorders.

Neuroimage., 31, 1614-1624.

Bishop, D. V. (1993). Annotation: autism, executive functions and theory of mind: a neuropsychological perspective. J.Child Psychol.Psychiatry, 34, 279-293.

Blaney, R. L. \& Winograd, E. (1978). Developmental differences in children's recognition memory for faces. Dev.Psychol., 14, 441-442.

Blomquist, H. K., Bohman, M., Edvinsson, S. O., Gillberg, C., Gustavson, K. H., Holmgren, G. et al. (1985). Frequency of the fragile X syndrome in infantile autism. A Swedish multicenter study. Clin.Genet., 27, 113-117.

Bloom, P. \& German, T. P. (2000). Two reasons to abandon the false belief task as a test of theory of mind. Cognition, 77, B25-B31.

Bodamer, J. (1947). Die-Prosop-agnosie. Arch.Psychiat.Nerv., 179, 6-54.

Bolton, P., Macdonald, H., Pickles, A., Rios, P., Goode, S., Crowson, M. et al. (1994). A case-control family history study of autism. J.Child Psychol.Psychiatry, 35, 877-900.

Bolton, P. F. \& Griffiths, P. D. (1997). Association of tuberous sclerosis of temporal lobes with autism and atypical autism. Lancet, 349, 392-395.

Boucher, J. \& Lewis, V. (1992). Unfamiliar face recognition in relatively able autistic children. J.Child Psychol.Psychiatry, 33, 843-859.

Boucher, J., Lewis, V., \& Collis, G. (1998). Familiar face and voice matching and recognition in children with autism. J.Child Psychol.Psychiatry, 39, 171-181.

Boutet, I. \& Chaudhuri, A. (2001). Multistability of overlapped face stimuli is dependent upon orientation. Perception, 30, 743-753.

Bowler, D. (2007). Autism Spectrum Disorders: Psychological Theory and Research. Chichester: Wiley.

Brand-D'Abrescia, M. \& Lavie, N. (2007). Distractor effects during processing of words under load. Psychon.Bull.Rev., 14, 1153-1157.

Brian, J. A., Tipper, S. P., Weaver, B., \& Bryson, S. E. (2003). Inhibitory mechanisms in autism spectrum disorders: typical selective inhibition of location versus facilitated perceptual processing. J.Child Psychol.Psychiatry, 44, 552-560. 
Broadbent, D. (1958). Perception and communication. Oxford: Oxford University Press.

Broadbent, D., Cooper, P. F., FitzGerald, P., \& Parkes, K. R. (1982). The cognitive failures questionnaire and its correlates. Br.J.Clin.Psychol., 21, 1-16.

Bruce, V. \& Young, A. (1986). Understanding face recognition. Br.J.Psychol., 77 ( Pt 3), 305-327.

Bryson, S. E., Wainwright-Sharp, J. A., \& Smith, L. M. (1990). Autism: A developmental spatial neglect syndrome? In J.Enns (Ed.), The development of attention: research and theory (pp. 405-427). North Holland: Elsevier.

Buchsbaum, M. S., Siegel, B. V., Jr., Wu, J. C., Hazlett, E., Sicotte, N., Haier, R. et al. (1992). Brief report: attention performance in autism and regional brain metabolic rate assessed by positron emission tomography. J.Autism Dev.Disord., 22, 115-125.

Burack, J., Enns, J., Stauder, J. E., Mottron, L., \& Randolf, B. (1997). Attention and autism: behavioral and electrophysical evidence. In D.J.Cohen \& F. R. Volkmar (Eds.), Handbook of autism and pervasive developmental disorders (pp. 226-247). New York: John Wiley \& Sons, Inc. autism. In.

Burack, J. \& Iarocci, G. (1995). Visual filtering and covert orienting in

Burack, J., Joseph, S., Russo, N., Shore, D. I., Porporino, M., \& Enns, J. (2009a). Change detection in naturalistic pictures among children with autism. J.Autism Dev.Disord., 39, 471-479.

Burack, J. A. (1994). Selective attention deficits in persons with autism: preliminary evidence of an inefficient attentional lens. J.Abnorm.Psychol., 103, 535543.

Burack, J. A., Iarocci, G., Mottron, L., Stauder, J. E., Robaey, P., \& Brennan, J. M. (1996). Visual filtering in persons with autism: A developmental perspective. In 26th International Congress of Psychology.

Burack, J. A., Joseph, S., Russo, N., Shore, D. I., Porporino, M., \& Enns, J. T. (2009b). Change detection in naturalistic pictures among children with autism.

J.Autism Dev.Disord., 39, 471-479.

Carey, S. \& Diamond, R. (1977). From piecemeal to configurational representation of faces. Science, 195, 312-314.

Carey, S. \& Diamond, R. (1994). Are faces perceived as configurations more by adults than by children? Visual Cognition, 1, 253-274.

Carey, S., Diamond, R., \& Woods, B. (1980). Development of face recognition - A maturational component? Dev.Psychol., 16, 257-269. 
Carper, R. A. \& Courchesne, E. (2000). Inverse correlation between frontal lobe and cerebellum sizes in children with autism. Brain, 123 ( Pt 4), 836-844.

Carper, R. A. \& Courchesne, E. (2005). Localized enlargement of the frontal cortex in early autism. Biol.Psychiatry, 57, 126-133.

Cartwright-Finch, U. \& Lavie, N. (2007). The role of perceptual load in inattentional blindness. Cognition, 102, 321-340.

Casey, B. J., Gordon, C. T., Mannheim, G. B., \& Rumsey, J. M. (1993). Dysfunctional attention in autistic savants. J.Clin.Exp.Neuropsychol., 15, 933-946.

Castelli, F. (2005). Understanding emotions from standardized facial expressions in autism and normal development. Autism, 9, 428-449.

Celani, G., Battacchi, M. W., \& Arcidiacono, L. (1999). The understanding of the emotional meaning of facial expressions in people with autism. J.Autism Dev.Disord., 29, 57-66.

Charman, T. \& Swettenham, J. (2001). Repetitive behaviours and socialcommunicative impairments in autism: implications for developmental theory and diagnosis. In J.Burack, T. Charman, N. Yirmiya, \& P. D. Zelazo (Eds.), The Development of Autism: Perspectives from Theory and Research (pp. 325-345). Hillsdale, New Jersey: Lawrence Erlbaum.

Chelune, G. J., Ferguson, W., Koon, R., \& Dickey, T. O. (1986). Frontal lobe disinhibition in attention deficit disorder. Child Psychiatry Hum.Dev., 16, 221-234.

Cherry, E. C. (1953). Some experiments on the recognition of speech with one and with two ears. Journal of the Acoustical Society of America, 25, 975-979.

Chung, M. S. \& Thomson, D. M. (1995). Development of face recognition. Br.J.Psychol., 86 ( Pt 1), 55-87.

Ciesielski, K. T., Courchesne, E., \& Elmasian, R. (1990). Effects of focused selective attention tasks on event-related potentials in autistic and normal individuals. Electroencephalogr.Clin.Neurophysiol., 75, 207-220.

Ciesielski, K. T., Knight, J. E., Prince, R. J., Harris, R. J., \& Handmaker, S. D. (1995). Event-related potentials in cross-modal divided attention in autism. Neuropsychologia, 33, 225-246.

Cohen, I. L., Sudhalter, V., Pfadt, A., Jenkins, E. C., Brown, W. T., \& Vietze, P. M. (1991). Why are autism and the fragile-X syndrome associated? Conceptual and methodological issues. Am.J.Hum.Genet., 48, 195-202.

Conway, A. R., Cowan, N., Bunting, M. F., Therriault, D. J., \& Minkoff, S. R. B. (2002). A latent variable analysis of working memory capacity, short term memory capacity, processing speed, and general fluid intelligence. Intelligence, 30, 163-183. 
Cook, E. H. \& Leventhal, B. L. (1996). The serotonin system in autism. Curr.Opin.Pediatr., 8, 348-354.

Corteen, R. S. \& Dunn, D. (1974). Shock-associated words in a nonattended message: a test for momentary awareness. J.Exp.Psychol., 102, 1143-1144.

Corteen, R. S. \& Wood, B. (1972). Autonomic responses to shock-associated words in an unattended channel. J.Exp.Psychol., 94, 308-313.

Courchesne, E. (1997). Brainstem, cerebellar and limbic neuroanatomical abnormalities in autism. Curr.Opin.Neurobiol., 7, 269-278.

Courchesne, E., Press, G. A., \& Yeung-Courchesne, R. (1993). Parietal lobe abnormalities detected with MR in patients with infantile autism. AJR

Am.J.Roentgenol., 160, 387-393.

Courchesne, E., Townsend, J., Akshoomoff, N. A., Saitoh, O., YeungCourchesne, R., Lincoln, A. J. et al. (1994). Impairment in shifting attention in autistic and cerebellar patients. Behav.Neurosci., 108, 848-865.

Courchesne, E., Yeung-Courchesne, R., Press, G. A., Hesselink, J. R., \& Jernigan, T. L. (1988). Hypoplasia of cerebellar vermal lobules VI and VII in autism. N.Engl.J.Med., 318, 1349-1354.

Cowan, N. (1995). Sensory memory and its role in information processing. Electroencephalogr.Clin.Neurophysiol.Suppl, 44, 21-31.

Cowan, N. (2001). The magical number 4 in short-term memory: a reconsideration of mental storage capacity. Behav.Brain Sci., 24, 87-114.

Cowan, N. (2008). What are the differences between long-term, short-term, and working memory? Prog.Brain Res., 169, 323-338.

Critchley, H. D., Daly, E. M., Bullmore, E. T., Williams, S. C., van, A. T., Robertson, D. M. et al. (2000). The functional neuroanatomy of social behaviour: changes in cerebral blood flow when people with autistic disorder process facial expressions. Brain, 123 ( Pt 11), 2203-2212.

Dalton, K. M., Nacewicz, B. M., Johnstone, T., Schaefer, H. S., Gernsbacher, M. A., Goldsmith, H. H. et al. (2005). Gaze fixation and the neural circuitry of face processing in autism. Nat.Neurosci., 8, 519-526.

Damasio, A. R. \& Maurer, R. G. (1978). A neurological model for childhood autism. Arch.Neurol., 35, 777-786.

Dapretto, M., Davies, M. S., Pfeifer, J. H., Scott, A. A., Sigman, M., Bookheimer, S. Y. et al. (2006). Understanding emotions in others: mirror neuron dysfunction in children with autism spectrum disorders. Nat.Neurosci., 9, 28-30.

Davis, G. \& Driver, J. (1998). Kanizsa subjective figures can act as occluding surfaces at parallel stages of visual search. J.Exp.Psychol.Hum.Percept.Perform., 24, 169-184. 
Dawson, G. \& Lewy, A. (1989). Arousal, Attention, and the Socioemotional Impairments of Individuals with Autism. In G.Dawson (Ed.), Autism, Nature, Diagnosis \& Treatment (pp. 49-74). New York: The Guilford Press.

Dawson, G., Toth, K., Abbott, R., Osterling, J., Munson, J., Estes, A. et al. (2004a). Early social attention impairments in autism: social orienting, joint attention, and attention to distress. Dev.Psychol., 40, 271-283.

Dawson, G., Webb, S., Schellenberg, G. D., Dager, S., Friedman, S., Aylward, E. et al. (2002). Defining the broader phenotype of autism: genetic, brain, and behavioral perspectives. Dev.Psychopathol., 14, 581-611.

Dawson, G., Webb, S. J., Carver, L., Panagiotides, H., \& McPartland, J. (2004b). Young children with autism show atypical brain responses to fearful versus neutral facial expressions of emotion. Dev.Sci., 7, 340-359.

Dawson, G., Webb, S. J., \& McPartland, J. (2005). Understanding the nature of face processing impairment in autism: insights from behavioral and electrophysiological studies. Dev.Neuropsychol., 27, 403-424.

de Fockert, J. W., Rees, G., Frith, C. D., \& Lavie, N. (2001). The role of working memory in visual selective attention. Science, 291, 1803-1806.

Dennis, M. (1991). Frontal lobe function in childhood and adolescence : a heuristic for assessing attention regulation, executive control, and the intentional states important for social discourse. Dev.Neuropsychol., 7, 327-358.

Deruelle, C., Rondan, C., Gepner, B., \& Tardif, C. (2004). Spatial frequency and face processing in children with autism and Asperger syndrome. J.Autism Dev.Disord., 34, 199-210.

Deutsch, J. A. \& Deutsch, D. (1963). Attention: some theoretical considerations. Psychological Review, 87, 272-300.

Duchaine, B. C., Dingle, K., Butterworth, E., \& Nakayama, K. (2004). Normal greeble learning in a severe case of developmental prosopagnosia. Neuron, 43, 469-473.

Duncan, J. (1986). Disorganization of behaviour after frontal lobe damage. Cognit.Neuropsychol., 3, 271-290.

Ellis, A. W. \& Young, A. W. (1988). Human Cognitive Neuropsychology. Hove,UK: Erlbaum.

Ellis, A. W., Young, A. W., \& Flude, B. M. (1990). Repetition priming and face processing: priming occurs within the system that responds to the identity of a face. Q.J.Exp.Psychol.A, 42, 495-512.

Enns, J. T. \& Akhtar, N. (1989). A developmental study of filtering in visual attention. Child Dev., 60, 1188-1199. 
Enright, S. J. \& Beech, A. R. (1993). Reduced cognitive inhibition in obsessive-compulsive disorder. Br.J.Clin.Psychol., 32 ( Pt 1), 67-74.

Eriksen, B. A. \& Eriksen, C. W. (1974). Effects of noise letters upon the identification of a target letter in a non search task. Percept.Psychophys., 16, 143149.

Farah, M. J. (1990). Visual agnosia: disorders of object recognition and what they tell us about normal vision. Cambridge,MA: MIT Press.

Farah, M. J., Rabinowitz, C., Quinn, G., \& Liu, G. T. (2000). Early committment of neural substrates for face recognition. Cognit.Neuropsychol., 1-3, 117-123.

Farah, M. J., Wilson, K. D., Drain, H. M., \& Tanaka, J. R. (1995). The inverted face inversion effect in prosopagnosia: evidence for mandatory, facespecific perceptual mechanisms. Vision Res., 35, 2089-2093.

Fletcher, P. C., Happe, F., Frith, U., Baker, S. C., Dolan, R. J., Frackowiak, R. S. et al. (1995). Other minds in the brain: a functional imaging study of "theory of mind" in story comprehension. Cognition, 57, 109-128.

Fletcher-Watson, S., Leekam, S. R., Turner, M. A., \& Moxon, L. (2006). Do people with autistic spectrum disorder show normal selection for attention? Evidence from change blindness. Br.J.Psychol., 97, 537-554.

Flin, R. H. (1980). Age effects in children's memory for unfamiliar faces. Dev.Psychol., 16, 374.

Fodor, J. (1983). The modularity of mind. Cambridge,MA: MIT Press.

Folstein, S. \& Rutter, M. (1977). Infantile autism: a genetic study of 21 twin pairs. J.Child Psychol.Psychiatry, 18, 297-321.

Fombonne, E. (2003). The prevalence of autism. JAMA, 289, 87-89.

Fombonne, E. (2009). Epidemiology of pervasive developmental disorders. Pediatr.Res., 65, 591-598.

Fombonne, E., Roge, B., Claverie, J., Courty, S., \& Fremolle, J. (1999). Microcephaly and macrocephaly in autism. J.Autism Dev.Disord., 29, 113-119.

Forster, S. \& Lavie, N. (2007). High perceptual load makes everybody equal: eliminating individual differences in distractibility with load. Psychol.Sci., 18, 377381.

Franklin, A., Sowden, P., Burley, R., Notman, L., \& Alder, E. (2008). Color perception in children with autism. J.Autism Dev.Disord., 38, 1837-1847.

Frith, C. D. \& Frith, U. (1999). Interacting minds--a biological basis. Science, 286, 1692-1695. 
Frith, U. (1989). A new look at language and communication in autism. Br.J.Disord.Commun., 24, 123-150.

Frith, U. \& Snowling, M. (1983). Reading for meaning and reading for sound in autistic and dyslexic children. British Journal of Developmental Psychology, 1, 329-342.

Gaffney, G. R., Kuperman, S., Tsai, L. Y., \& Minchin, S. (1989). Forebrain structure in infantile-autism. Journal of the American Academy of Child and Adolescent Psychiatry, 28, 534-537.

Gallagher, H. L., Happe, F., Brunswick, N., Fletcher, P. C., Frith, U., \& Frith, C. D. (2000). Reading the mind in cartoons and stories: an fMRI study of 'theory of mind' in verbal and nonverbal tasks. Neuropsychologia, 38, 11-21.

Gallese, V. (2003). The roots of empathy: the shared manifold hypothesis and the neural basis of intersubjectivity. Psychopathology, 36, 171-180.

Gallese, V., Keysers, C., \& Rizzolatti, G. (2004). A unifying view of the basis of social cognition. Trends Cogn Sci., 8, 396-403.

Garretson, H. B., Fein, D., \& Waterhouse, L. (1990). Sustained attention in children with autism. J.Autism Dev.Disord., 20, 101-114.

Gauthier, I. \& Logothetis, N. K. (2000). Is face recognition not so unique after all? Cognit.Neuropsychol., 17, 125-142.

Gauthier, I., Skudlarski, P., Gore, J. C., \& Anderson, A. W. (2000). Expertise for cars and birds recruits brain areas involved in face recognition. Nat.Neurosci., 3, 191-197.

Gauthier, I., Tarr, M. J., Anderson, A. W., Skudlarski, P., \& Gore, J. C. (1999). Activation of the middle fusiform 'face area' increases with expertise in recognizing novel objects. Nat.Neurosci., 2, 568-573.

George, M., Costa, D., Kouris, K., \& Ring, H. E. P. (1992). Cerebral blood flow abnormalities in adults with infantile autism. Journal of Nervous and Mental Diseases, 188, 413-417.

Gepner, B., Deruelle, C., \& Grynfeltt, S. (2001). Motion and emotion: a novel approach to the study of face processing by young autistic children. J.Autism Dev.Disord., 31, 37-45.

Gillberg, C. (1983). Identical triplets with infantile autism and the fragile-X syndrome. Br.J.Psychiatry, 143, 256-260.

Gillberg, C. (1991). The treatment of epilepsy in autism. J.Autism Dev.Disord., 21, 61-77.

Gillberg, C. (1998). Chromosomal disorders and autism. J.Autism Dev.Disord., 28, 415-425. 
Gillberg, C. \& Coleman, M. (1992). The biology of autistic syndromes. Oxford: MacKeith.

Gillberg, C. \& Wahlstrom, J. (1985). Chromosome abnormalities in infantile autism and other childhood psychoses: a population study of 66 cases.

Dev.Med.Child Neurol., 27, 293-304.

Gillberg, C., Wahlstrom, J., \& Hagberg, B. (1984a). Infantile autism and Rett's syndrome: common chromosomal denominator. Lancet, 2, 1094-1095.

Gillberg, C., Wahlstrom, J., \& Hagberg, B. (1985). A "new" chromosome marker common to the Rett syndrome and infantile autism? The frequency of fragile sites at X p22 in 81 children with infantile autism, childhood psychosis and the Rett syndrome. Brain Dev., 7, 365-367.

Gillberg, C., Winnergard, I., \& Wahlstrom, J. (1984b). The sex chromosomes--one key to autism? An XYY case of infantile autism.

Appl.Res.Ment.Retard., 5, 353-360.

Gladstone, M., Carter, A. S., Schultz, R. T., Riddle, M., Schahill, L., \& Pauls, D. L. (1993). Neuropsychological functioning of children affected with Tourette's Syndrome and obsessive-compulsive disorder. In International Neuropsychological Society Galveston, TX: International Neuropsychological Society.

Gold, M. S. \& Gold, J. R. (1974). Autism and attention: theoretical considerations and a pilot study using set reaction time. Child Psychiatry and Human Development, 6, 68-80.

Goldberg, T. E., Maltz, A., Bow, J. N., Karson, C. N., \& Leleszi, J. P. (1987). Blink rate abnormalities in autistic and mentally retarded children: relationship to dopaminergic activity. J.Am.Acad.Child Adolesc.Psychiatry, 26, 336-338.

Goldstein, G., Johnson, C. R., \& Minshew, N. J. (2001). Attentional processes in autism. J.Autism Dev.Disord., 31, 433-440.

Goodman, R. (1989). Infantile autism: a syndrome of multiple primary deficits? J.Autism Dev.Disord., 19, 409-424.

Goren, C. C., Sarty, M., \& Wu, P. Y. (1975). Visual following and pattern discrimination of face-like stimuli by newborn infants. Pediatrics, 56, 544-549.

Gorno-Tempini, M. L. \& Price, C. J. (2001). Identification of famous faces and buildings: a functional neuroimaging study of semantically unique items. Brain, 124, 2087-2097.

Gorno-Tempini, M. L., Price, C. J., Josephs, O., Vandenberghe, R., Cappa, S. F., Kapur, N. et al. (1998). The neural systems sustaining face and proper-name processing. Brain, 121 (Pt 11), 2103-2118.

Grandin, T. (1986). Emergence: Labelled Autistic. Novato,CA: Arena Press. 
Grandin, T. (1995). Thinking in pictures and other reports from my life with autism. New York: Doubleday.

Grant, D. \& Berg, E. (1948). A Behavioral Analysis of Degree of Reinforcement and Ease of Shifting to New Responses in a Weigl-Type Card-Sorting Problem. J.Exp.Psychol., 38, 404-411.

Grattan, L. M. \& Eslinger, P. J. (1989). Empirical study of empathy. Am.J.Psychiatry, 146, 1521-1522.

Grattan, L. M. \& Eslinger, P. J. (1992). Long-term psychological consequences of childhood frontal lobe lesion in patient DT. Brain Cogn, 20, 185195.

Graveling, R. A. \& Brooke, J. D. (1978). Hormonal and cardiac response of autistic children to changes in environmental stimulation. J.Autism Child Schizophr., $8,441-455$.

Greenaway, R. \& Plaisted, K. (2005). Top-down attentional modulation in autistic spectrum disorders is stimulus-specific. Psychol.Sci., 16, 987-994.

Grelotti, D. J., Gauthier, I., \& Schultz, R. T. (2002). Social interest and the development of cortical face specialization: what autism teaches us about face processing. Dev.Psychobiol., 40, 213-225.

Grice, SJ., Spratling, M. W., Karmiloff-Smith, A., Halit, H., Csibra, G., de Haan, M. et al. (2001). Disordered visual processing and oscillatory brain activity in autism and Williams syndrome. Neuroreport, 12, 2697-2700.

Gross, T. F. (2004). The perception of four basic emotions in human and nonhuman faces by children with autism and other developmental disabilities. J.Abnorm.Child Psychol., 32, 469-480.

Grossman, J. B., Klin, A., Carter, A. S., \& Volkmar, F. (2000). Verbal bias in recognition of facial emotions in children with Asperger syndrome. J.Child Psychol.Psychiatry, 41, 369-379.

Haas, R. H., Townsend, J., Courchesne, E., Lincoln, A. J., Schreibman, L., \& Yeung-Courchesne, R. (1996). Neurologic abnormalities in infantile autism. J.Child Neurol., 11, 84-92.

Hadjikhani, N., Joseph, R. M., Snyder, J., Chabris, C. F., Clark, J., Steele, S. et al. (2004). Activation of the fusiform gyrus when individuals with autism spectrum disorder view faces. Neuroimage., 22, 1141-1150.

Hadjikhani, N., Joseph, R. M., Snyder, J., \& Tager-Flusberg, H. (2006). Anatomical differences in the mirror neuron system and social cognition network in autism. Cereb.Cortex, 16, 1276-1282.

Hadjikhani, N., Joseph, R. M., Snyder, J., \& Tager-Flusberg, H. (2007). Abnormal activation of the social brain during face perception in autism. Hum.Brain Mapp., 28, 441-449. 
Hallmayer, J., Hebert, J. M., Spiker, D., Lotspeich, L., McMahon, W. M., Petersen, P. B. et al. (1996). Autism and the X chromosome. Multipoint sib-pair analysis. Arch.Gen.Psychiatry, 53, 985-989.

Happe, F. (1991). Theory of Mind and Communication in Autism. $\mathrm{PhD}$ University of London.

Happe, F. (1999). Autism: cognitive deficit or cognitive style? Trends Cogn Sci., 3, 216-222.

Happe, F., Ronald, A., \& Plomin, R. (2006). Time to give up on a single explanation for autism. Nat.Neurosci., 9, 1218-1220.

Happe, F. G. (1994). An advanced test of theory of mind: understanding of story characters' thoughts and feelings by able autistic, mentally handicapped, and normal children and adults. J.Autism Dev.Disord., 24, 129-154.

Happe, F. G. (1995). The role of age and verbal ability in the theory of mind task performance of subjects with autism. Child Dev., 66, 843-855.

Harris, N. S., Courchesne, E., Townsend, J., Carper, R. A., \& Lord, C. (1999). Neuroanatomic contributions to slowed orienting of attention in children with autism. Brain Res.Cogn Brain Res., 8, 61-71.

Hashimoto, T., Tayama, M., Mori, K., Fujino, K., Miyazaki, M., \& Kuroda, Y. (1989). Magnetic resonance imaging in autism: preliminary report.

Neuropediatrics, 20, 142-146.

Hauck, M., Fein, D., Maltby, N., Waterhouse, L., \& Feinstein, C. (1998). Memory for faces in children with autism. Child Neuropsychol., 4, 187-198.

Haxby, J. V., Hoffman, E. A., \& Gobbini, M. I. (2000). The distributed human neural system for face perception. Trends Cogn Sci., 4, 223-233.

Haxby, J. V., Horwitz, B., Ungerleider, L. G., Maisog, J. M., Pietrini, P., \& Grady, C. L. (1994). The functional organization of human extrastriate cortex: a PET-rCBF study of selective attention to faces and locations. J.Neurosci., 14, 63366353.

Hayes, R. (1987). Training for work. In D.J.Cohen \& A. M. Donnellan (Eds.), Handbook of Autism and Pervasive Developmental Disorders ( Silver Springs, MD: Winston.

Head, D., Bolton, D., \& Hymas, N. (1989). Deficit in cognitive shifting ability in patients with obsessive-compulsive disorder. Biol.Psychiatry, 25, 929-937.

Heh, C. W., Smith, R., Wu, J., Hazlett, E., Russell, A., Asarnow, R. et al. (1989). Positron emission tomography of the cerebellum in autism. Am.J.Psychiatry, 146, 242-245. 
Heilman, K., Watson, R., \& Valenstein, E. (1993). Neglect and related disorders. In K.Heilman \& E. Valenstein (Eds.), Clinical Neuropsychology (3rd ed., New York: Oxford University Press.

Hermelin, B. \& O'Connor, N. (1970). Psychological experiments with autistic children. Oxford: Pergamon Press.

Hill, E. L. \& Bird, C. M. (2006). Executive processes in Asperger syndrome: patterns of performance in a multiple case series. Neuropsychologia, 44, 2822-2835.

Hismjatullina, A. (2006). A study of selective attention in young autistic subjects. PhD University of Missouri.

Hobson, R. P. (1986a). The autistic child's appraisal of expressions of emotion. J.Child Psychol.Psychiatry, 27, 321-342.

Hobson, R. P. (1986b). The autistic child's appraisal of expressions of emotion: a further study. J.Child Psychol.Psychiatry, 27, 671-680.

Hobson, R. P., Ouston, J., \& Lee, A. (1988). What's in a face? The case of autism. Br.J.Psychol., 79 ( Pt 4), 441-453.

Hoon, A. H., Jr. \& Reiss, A. L. (1992). The mesial-temporal lobe and autism: case report and review. Dev.Med.Child Neurol., 34, 252-259.

Horowitz, T., Wolfe, J., Keehn, B., Connolly, C., \& Joseph, R. (2007). Is superior visual search in autism due to memory in search? Journal of Vision 7[9], 712a.

Ref Type: Abstract

Hossein Fatemi, S., Halt, A. R., Realmuto, G., Earle, J., Kist, D. A., Thuras, P. et al. (2002). Purkinje cell size is reduced in cerebellum of patients with autism. Cellular and Molecular Neurobiology, 22, 171-175.

Huang, L. \& Pashler, H. (2005). Attention capacity and task difficulty in visual search. Cognition, 94, B101-B111.

Huang-Pollock, C. L., Carr, T. H., \& Nigg, J. T. (2002). Development of selective attention: perceptual load influences early versus late attentional selection in children and adults. Dev.Psychol., 38, 363-375.

Hubl, D., Bolte, S., Feineis-Matthews, S., Lanfermann, H., Federspiel, A., Strik, W. et al. (2003). Functional imbalance of visual pathways indicates alternative face processing strategies in autism. Neurology, 61, 1232-1237.

Hughes, C. \& Russell, J. (1993). Autistic children's difficulties with mental disengagement from an object: Its implications for theories of Autism. Dev.Psychol., $29,498-510$.

Hughes, C., Russell, J., \& Robbins, T. W. (1994). Specific planning deficit in autism: Evidence for a central executive dysfunction. Neuropsychologia, 32, 477492. 
Hutt, C., Hutt, S. J., Lee, D., \& Ounsted, C. (1964). Arousal and childhood autism. Nature, 204, 908-909.

Hutt, S. J., Hutt, C., Lee, D., \& Ounsted, C. (1965). A behavioural and electroencephalographic study of autistic children. J.Psychiatr.Res., 3, 181-197.

Iarocci, G. \& Burack, J. (2005). The visual orienting system in people with autism: Clues about how it functions. In.

Iarocci, G. \& Burack, J. A. (2004). Intact covert orienting to peripheral cues among children with autism. J.Autism Dev.Disord., 34, 257-264.

James, W. A. (1890). The priciples of psychology. New York: Dover.

Jeffreys, D. A. \& Tukmachi, E. S. (1992). The vertex-positive scalp potential evoked by faces and by objects. Exp.Brain Res., 91, 340-350.

Jemel, B., Mottron, L., \& Dawson, M. (2006). Impaired face processing in autism: fact or artifact? J.Autism Dev.Disord., 36, 91-106.

Jenkins, R., Burton, A. M., \& Ellis, A. W. (2002). Long-term effects of covert face recognition. Cognition, 86, B43-52.

Jenkins, R., Lavie, N., \& Driver, J. (2003). Ignoring famous faces: categoryspecific dilution of distractor interference. Percept.Psychophys., 65, 298-309.

Johnson, M. H. (2005). Subcortical face processing. Nature Rev.Neurosci., 6, 766-774.

Johnson, M. H., Dziurawiec, S., Ellis, H., \& Morton, J. (1991). Newborns' preferential tracking of face-like stimuli and its subsequent decline. Cognition, 40, 119.

Johnson, M. H. \& Morton, J. (1991). Biology and cognitive development: The case of face recognition. Oxford,UK: Blackwell.

Johnston, J. C. \& McClelland, J. L. (1974). Perception of letters in words: seek not and ye shall find. Science, 184, 1192-1194.

Jolliffe, T. \& Baron-Cohen, S. (1997). Are people with autism and Asperger syndrome faster than normal on the Embedded Figures Test? J.Child Psychol.Psychiatry, 38, 527-534.

Joseph, R., Keehn, B., Connolly, C., Wolfe, J., \& Horowitz, T. (2009). Why is visual search superior in autism spectrum disorder? Dev.Sci., [Epub ahead of print].

Joseph, R. M. \& Tager-Flusberg, H. (2004). The relationship of theory of mind and executive functions to symptom type and severity in children with autism. Dev.Psychopathol., 16, 137-155. 
Joseph, R. M. \& Tanaka, J. (2003). Holistic and part-based face recognition in children with autism. J.Child Psychol.Psychiatry, 44, 529-542.

Just, M. A., Cherkassky, V. L., Keller, T. A., Kana, R. K., \& Minshew, N. J. (2007). Functional and anatomical cortical underconnectivity in autism: evidence from an FMRI study of an executive function task and corpus callosum morphometry. Cereb.Cortex, 17, 951-961.

Kahneman, D., Treisman, A., \& Gibbs, B. J. (1992). The reviewing of object files: object-specific integration of information. Cogn Psychol., 24, 175-219.

Kanner, L. (1943). Autistic disturbances of affective contact. Nervous Child, 2, 217-250.

Kanwisher, N. (2000). Domain specificity in face perception. Nat.Neurosci., 3, 759-763.

Kanwisher, N., McDermott, J., \& Chun, M. M. (1997). The fusiform face area: a module in human extrastriate cortex specialized for face perception. J.Neurosci., 17, 4302-4311.

Kemner, C., van Ewijk, L., van Engeland, H., \& Hooge, I. (2008). Brief report: Eye movements during visual search tasks indicate enhanced stimulus discriminability in subjects with PDD. Journal of Autism and Developmental Disorders, 38, 553-557.

Kemper, T. L. \& Bauman, M. L. (1993). The contribution of neuropathologic studies to the understanding of autism. Neurol.Clin., 11, 175-187.

Kinsbourne, M. (1987). Brain mechanisms and memory. Hum.Neurobiol., 6, $81-92$.

Kleiner, K. A. \& Banks, M. S. (1987). Stimulus energy does not account for 2-month-olds' face preferences. J.Exp.Psychol.Hum.Percept.Perform., 13, 594-600.

Klin, A., Jones, W., Schultz, R., Volkmar, F., \& Cohen, D. (2002). Visual fixation patterns during viewing of naturalistic social situations as predictors of social competence in individuals with autism. Arch.Gen.Psychiatry, 59, 809-816.

Klin, A., Sparrow, S. S., de, B. A., Cicchetti, D. V., Cohen, D. J., \& Volkmar, F. R. (1999). A normed study of face recognition in autism and related disorders. J.Autism Dev.Disord., 29, 499-508.

Kovattana, P. M. \& Kraemer, H. C. (1974). Response to multiple visual cues of color, size, and form by autistic children. J.Autism Child Schizophr., 4, 251-261.

Kuperman, S., Beeghly, J. H., Burns, T. L., \& Tsai, L. Y. (1985). Serotonin relationships of autistic probands and their first-degree relatives. J.Am.Acad.Child Psychiatry, 24, 186-190.

LaBerge, D. (1983). Spatial extent of attention to letters and words. J.Exp.Psychol.Hum.Percept.Perform., 371-379. 
Lahaie, A., Mottron, L., Arguin, M., Berthiaume, C., Jemel, B., \& Saumier, D. (2006). Face perception in high-functioning autistic adults: evidence for superior processing of face parts, not for a configural face-processing deficit.

Neuropsychology., 20, 30-41.

Lainhart, J. E., Bigler, E. D., Bocian, M., Coon, H., Dinh, E., Dawson, G. et al. (2006). Head circumference and height in autism: a study by the Collaborative Program of Excellence in Autism. Am.J.Med.Genet.A, 140, 2257-2274.

Landry, R. \& Bryson, S. E. (2004). Impaired disengagement of attention in young children with autism. J.Child Psychol.Psychiatry, 45, 1115-1122.

Langdell, T. (1978). Recognition of faces: An approach to the study of autism. J.Child Psychol.Psychiatry, 19, 255-268.

Lavie, N. (1995). Perceptual load as a necessary condition for selective attention. J.Exp.Psychol.Hum.Percept.Perform., 21, 451-468.

Lavie, N. (2000). Selective attention and cognitive control: dissociating attentional functions through different types of load. In S.Monsell \& J. Driver (Eds.), Control of cognitive processes. Attention and perfomance XVIII (pp. 175-194). Cambridge,MA: The MIT Press.

Lavie, N. \& Cox, S. (1997). On the efficiency of visual selective attention: efficient visual search leads to inefficient distractor rejection. Psychol.Sci., 8, 395398.

Lavie, N. \& de Fockert, J. W. (2003). Contrasting effects of sensory limits and capacity limits in visual selective attention. Percept.Psychophys., 65, 202-212.

Lavie, N., Hirst, A., de Fockert, J. W., \& Viding, E. (2004a). Load theory of selective attention and cognitive control. J.Exp.Psychol.Gen., 133, 339-354.

Lavie, N., Hirst, A., de Fockert, J. W., \& Viding, E. (2004b). Load theory of selective attention and cognitive control. J.Exp.Psychol.Gen., 133, 339-354.

Lavie, N., Hirst, A., de Fockert, J. W., \& Viding, E. (2004c). Load theory of selective attention and cognitive control. J.Exp.Psychol.Gen., 133, 339-354.

Lavie, N., Ro, T., \& Russell, C. (2003). The role of perceptual load in processing distractor faces. Psychol.Sci., 14, 510-515.

Lavie, N. \& Tsal, Y. (1994). Perceptual load as a major determinant of the locus of selection in visual attention. Percept.Psychophys., 56, 183-197.

Le Grand, R., Mondloch, C. J., Maurer, D., \& Brent, H. P. (2001). Neuroperception. Early visual experiment and face processing. Nature, 410, 890.

Leekam, S. \& Moore, C. (2001). The development of attention and joint attention in children with autism. In J.Burack, T. Charman, N. Yirmiya, \& P. D. Zelazo (Eds.), The development of autism (pp. 105-130). Mawah,NJ: Erlbaum. 
Leekam, S. R., Lopez, B., \& Moore, C. (2000). Attention and joint attention in preschool children with autism. Dev.Psychol., 36, 261-273.

Leslie, A. M. \& Frith, U. (1987). Metarepresentation and autism: how not to lose one's marbles. Cognition, 27, 291-294.

Leslie, A. M. \& Happe, F. (1989). Autism and ostensive communication: The relevance of metarepresentation. Development and Psychopathology, 1, 205-212.

Leslie, K. R., Johnson-Frey, S. H., \& Grafton, ST. (2004). Functional imaging of face and hand imitation: towards a motor theory of empathy.

Neuroimage., 21, 601-607.

Liu, J., Nyholt, D. R., Magnussen, P., Parano, E., Pavone, P., Geschwind, D. et al. (2001). A genomewide screen for autism susceptibility loci. Am.J.Hum.Genet., $69,327-340$.

Lopez, B., Hadwin, J. A., Donnelly, N., \& Leekam, S. (2004). Face processing in high-functioning adolescents with autism: Evidence for weak central coherence? Visual Cognition, 11, 673-688.

Lopez, B. \& Leekam, S. R. (2003). Do children with autism fail to process information in context? J.Child Psychol.Psychiatry, 44, 285-300.

Lord, C. (1993). The Complexity of Social Behaviour in Autism. In S.BaronCohen, H. Tager-Flusberg, \& D. J. Cohen (Eds.), Understanding Other Minds: Perspectives from Autism (pp. 292-316). Oxford: Oxford University Press.

Lord, C., Rutter, M., DiLavore, P. C., \& Risi, S. (2002). Autism Diagnostic Observational Schedule. Los Angeles, CA: Western Psychological Services.

Lovaas, O. I., Koegel, R. L., \& Schreibman, L. (1979). Stimulus overselectivity in autism: a review of research. Psychol.Bull., 86, 1236-1254.

Lovaas, O. I., Schreibman, L., Koegel, R., \& Rehm, R. (1971). Selective responding by autistic children to multiple sensory input. J.Abnorm.Psychol., 77, 211-222.

Luck, S. J. \& Vogel, E. K. (1997). The capacity of visual working memory for features and conjunctions. Nature, 390, 279-281.

Macdonald, J. S. \& Lavie, N. (2008). Load induced blindness. J.Exp.Psychol.Hum.Percept.Perform., 34, 1078-1091.

Mann, T. A. \& Walker, P. (2003). Autism and a deficit in broadening the spread of visual attention. J.Child Psychol.Psychiatry, 44, 274-284.

Marcus, D. J. \& Nelson, C. A. (2001). Neural bases and development of face recognition in autism. CNS.Spectr., 6, 36-59.

Maylor, E. A. \& Lavie, N. (1998). The influence of perceptual load on age differences in selective attention. Psychol.Aging, 13, 563-573. 
Mazzocco, M. M., Kates, W. R., Baumgardner, T. L., Freund, L. S., \& Reiss, A. L. (1997). Autistic behaviors among girls with fragile X syndrome. J.Autism Dev.Disord., 27, 415-435.

McCarthy, G., Puce, A., Gore, J. C., \& Allison, T. (1997). Face-specific processing in the human fusiform gyrus. J.Cogn Neurosci., 9, 605-610.

McDougle, C. J., Naylor, S. T., Cohen, D. J., Aghajanian, G. K., Heninger, G. R., \& Price, L. H. (1996). Effects of tryptophan depletion in drug-free adults with autistic disorder. Arch.Gen.Psychiatry, 53, 993-1000.

McPartland, J., Dawson, G., Webb, S. J., Panagiotides, H., \& Carver, L. J. (2004). Event-related brain potentials reveal anomalies in temporal processing of faces in autism spectrum disorder. J.Child Psychol.Psychiatry, 45, 1235-1245.

Minshew, N. J., Luna, B., \& Sweeney, J. A. (1999). Oculomotor evidence for neocortical systems but not cerebellar dysfunction in autism. Neurology, 52, 917922.

Moray, N. P. (1959). Attention in dichotic listening: affective cues and the influence of instructions. Quarterly Journal of Experimental Psychology, 11, 56-60.

Moscovitch, M., Winocur, G., \& Behrmann, M. (1997). What is special about face recognition? Nineteen experiments on a person with visual object agnosia and dyslexia but normal face recognition. J.Cogn Neurosci., 9, 555-604.

Mottron, L. \& Belleville, S. (1993). A study of perceptual analysis in a highlevel autistic subject with exceptional graphic abilities. Brain Cogn, 23, 279-309.

Mottron, L. \& Burack, J. (2001). Enhanced perceptual functioning in the development of autism. In J.Burack, T. Charman, N. Yirmiya, \& P. D. Zelazo (Eds.), The Development of Autism: Perspectives from Theory and Research (pp. 131-148). Mahwah, NJ: Erlbaum.

Mottron, L., Burack, J. A., Iarocci, G., Belleville, S., \& Enns, J. T. (2003). Locally oriented perception with intact global processing among adolescents with high-functioning autism: evidence from multiple paradigms. J.Child Psychol.Psychiatry, 44, 904-913.

Mottron, L., Burack, J. A., Stauder, J. E., \& Robaey, P. (1999). Perceptual processing among high-functioning persons with autism. J.Child Psychol.Psychiatry, 40, 203-211.

Mottron, L., Dawson, M., Soulieres, I., Hubert, B., \& Burack, J. (2006). Enhanced perceptual functioning in autism: an update, and eight principles of autistic perception. J.Autism Dev.Disord., 36, 27-43.

Nagy, A. L. \& Sanchez, R. R. (1990). Critical color differences determined with a visual search task. J.Opt.Soc.Am.A, 7, 1209-1217. 
Nakamura, K., Kawashima, R., Ito, K., Sugiura, M., Kato, T., Nakamura, A. et al. (1999). Activation of the Right Inferior Frontal Cortex During Assessment of Facial Emotion. J.Neurophysiol., 82, 1610-1614.

Nakamura, K., Kawashima, R., Sato, N., Nakamura, A., Sugiura, M., Kato, T. et al. (2000). Functional delineation of the human occipito-temporal areas related to face and scene processing. A PET study. Brain, 123 ( Pt 9), 1903-1912.

Neisser, U. (1976). Cognition and reality: principles and implications of cognitive psychology. San Francisco: W.H. Freeman.

Nelson, C. A. (2001). The development and neural bases of face recognition. Inf.Child.Dev., 10, 3-18.

Nelson, H. (1982). The National Adult Reading Test. Windsor: NFER.

Ney, P. (1979). A psychopathogenesis of autism. Child Psychiatry and Human Development, 9, 195-205.

O'Connor, K., Hamm, J. P., \& Kirk, I. J. (2005). The neurophysiological correlates of face processing in adults and children with Asperger's syndrome. Brain Cogn, 59, 82-95.

O'Riordan, M. \& Plaisted, K. (2001a). Enhanced discrimination in autism. Q.J.Exp.Psychol.A, 54, 961-979.

O'Riordan, M. \& Plaisted, K. (2001b). Enhanced discrimination in autism. Q.J.Exp.Psychol.A, 54, 961-979.

O'Riordan, M. A. (2004). Superior visual search in adults with autism. Autism, 8, 229-248.

O'Riordan, M. A., Plaisted, K. C., Driver, J., \& Baron-Cohen, S. (2001). Superior visual search in autism. J.Exp.Psychol.Hum.Percept.Perform., 27, 719-730.

Onishi, K. H. \& Baillargeon, R. (2005). Do 15-month-old infants understand false beliefs? Science, 308, 255-258.

Ornitz, E. M. \& Ritvo, E. R. (1968). Neurophysiologic mechanisms underlying perceptual inconstancy in autistic and schizophrenic children. Archives of General Psychology, 18, 76-98.

Osterling, J. \& Dawson, G. (1994). Early recognition of children with autism: a study of first birthday home videotapes. J.Autism Dev.Disord., 24, 247-257.

Osterling, J. A., Dawson, G., \& Munson, J. A. (2002). Early recognition of 1year-old infants with autism spectrum disorder versus mental retardation. Dev.Psychopathol., 14, 239-251.

Owen, A. M., Roberts, A. C., Hodges, J. R., Summers, B. A., Polkey, C. E., $\&$ Robbins, T. W. (1995). Dopamine-dependent fronto-striatal planning deficits in early Parkinson's disease. Neuropsychology, 9, 126-140. 
Ozonoff, S., Cook, I., Coon, H., Dawson, G., Joseph, R. M., Klin, A. et al. (2004). Performance on Cambridge Neuropsychological Test Automated Battery subtests sensitive to frontal lobe function in people with autistic disorder: evidence from the Collaborative Programs of Excellence in Autism network. J.Autism Dev.Disord., 34, 139-150.

Ozonoff, S. \& McEvoy, R. E. (1994). A longitudinal study of executive function and theory of mind development in autism. Dev.Psychopathol., 6, 415-431.

Ozonoff, S. \& Miller, J. N. (1995). Teaching theory of mind: a new approach to social skills training for individuals with autism. J.Autism Dev.Disord., 25, 415433.

Ozonoff, S., Pennington, B. F., \& Rogers, S. (1990). Are there emotion perception deficits in young autistic children? J.Child Psychol.Psychiatry, 31, 343361.

Ozonoff, S., Pennington, B. F., \& Rogers, S. J. (1991). Executive function deficits in high-functioning autistic individuals: relationship to theory of mind. J.Child Psychol.Psychiatry, 32, 1081-1105.

Ozonoff, S. \& Strayer, D. L. (1997). Inhibitory function in nonretarded children with autism. J.Autism Dev.Disord., 27, 59-77.

Ozonoff, S., Strayer, D. L., McMahon, W. M., \& Filloux, F. (1994). Executive function abilities in autism and Tourette syndrome: an information processing approach. J.Child Psychol.Psychiatry, 35, 1015-1032.

Palermo, R. \& Rhodes, G. (2002). The influence of divided attention on holistic face perception. Cognition, 82, 225-257.

Pascalis, O., de Haan, M., \& Nelson, C. A. (2002). Is face processing speciesspecific during the first year of life? Science, 296, 1321-1323.

Pascualvaca, D. M., Fantie, B. D., Papageorgiou, M., \& Mirsky, A. F. (1998). Attentional capacities in children with autism: is there a general deficit in shifting focus? J.Autism Dev.Disord., 28, 467-478.

Pashler, H. (1988). Familiarity and visual change detection. Percept.Psychophys., 44, 369-378.

Pasto, L. \& Burack, J. A. (1995). A developmental study of visual filtering: Can windows facilitate filtering efficiency? In Annual meeting of the Canadian Psychological Association.

Pelphrey, K. A., Mack, P. B., Song, A., Guzeldere, G., \& McCarthy, G. (2003). Faces evoke spatially differentiated patterns of BOLD activation and deactivation. Neuroreport, 14, 955-959.

Pelphrey, K. A., Sasson, N. J., Reznick, J. S., Paul, G., Goldman, B. D., \& Piven, J. (2002). Visual scanning of faces in autism. J.Autism Dev.Disord., 32, 249261. 
Pennington, B. F. \& Ozonoff, S. (1996). Executive functions and developmental psychopathology. J.Child Psychol.Psychiatry, 37, 51-87.

Perner, J. (1995). The many faces of belief: reflections on Fodor's and the child's theory of mind. Cognition, 57, 241-269.

Perner, J., Frith, U., Leslie, A. M., \& Leekam, S. R. (1989). Exploration of the autistic child's theory of mind: knowledge, belief, and communication. Child Dev., 60, 688-700.

Perner, J. \& Wimmer, H. (1985). John thinks that Mary thinks that... Attribution of second-order beliefs by 5-to 10-year-old children. J.Exp.Child Psychol., 39, 437-471.

Phillips, W. A. (1974). On the distinction between sensory storage and shortterm visual memory. Percept.Psychophys., 16, 283-290.

Pickles, A., Bolton, P., Macdonald, H., Bailey, A., Le, C. A., Sim, C. H. et al. (1995). Latent-class analysis of recurrence risks for complex phenotypes with selection and measurement error: a twin and family history study of autism. Am.J.Hum.Genet., 57, 717-726.

Pierce, K., Glad, K. S., \& Schreibman, L. (1997). Social perception in children with autism: an attentional deficit? J.Autism Dev.Disord., 27, 265-282.

Pierce, K., Muller, R. A., Ambrose, J., Allen, G., \& Courchesne, E. (2001). Face processing occurs outside the fusiform 'face area' in autism: evidence from functional MRI. Brain, 124, 2059-2073.

Piven, J., Arndt, S., Bailey, J., Havercamp, S., Andreasen, N. C., \& Palmer, P. (1995). An MRI study of brain size in autism. Am.J.Psychiatry, 152, 1145-1149.

Piven, J., Tsai, G. C., Nehme, E., Coyle, J. T., Chase, G. A., \& Folstein, S. E. (1991). Platelet serotonin, a possible marker for familial autism. J.Autism Dev.Disord., 21, 51-59.

Plaisted, K., O'Riordan, M., \& Baron-Cohen, S. (1998a). Enhanced discrimination of novel, highly similar stimuli by adults with autism during a perceptual learning task. J.Child Psychol.Psychiatry, 39, 765-775.

Plaisted, K., O'Riordan, M., \& Baron-Cohen, S. (1998b). Enhanced visual search for a conjunctive target in autism: a research note. J.Child Psychol.Psychiatry, 39, 777-783.

Plaisted, K., O'Riordan, M., \& Baron-Cohen, S. (1998c). Enhanced visual search for a conjunctive target in autism: a research note. J.Child Psychol.Psychiatry, 39, 777-783.

Plaisted, K., Swettenham, J., \& Rees, L. (1999). Children with autism show local precedence in a divided attention task and global precedence in a selective attention task. J.Child Psychol.Psychiatry, 40, 733-742. 
Posner, M. I. \& Petersen, S. E. (1990). The attention system of the human brain. Annu.Rev.Neurosci., 13, 25-42.

Premack, D. \& Woodruff, G. (1978). Chimpanzee problem-solving: a test for comprehension. Science, 202, 532-535.

Prior, M. \& Hoffman, W. (1990). Brief report: Neuropsychological testing of autistic children through an exploration with frontal lobe tests. J.Autism Dev.Disord., 20, 581-590.

Puce, A., Allison, T., Asgari, M., Gore, J. C., \& McCarthy, G. (1996). Differential sensitivity of human visual cortex to faces, letterstrings, and textures: a functional magnetic resonance imaging study. J.Neurosci., 16, 5205-5215.

Puce, A., Allison, T., Bentin, S., Gore, J. C., \& McCarthy, G. (1998). Temporal cortex activation in humans viewing eye and mouth movements. J.Neurosci., 18, 2188-2199.

Raymaekers, R., van der, M. J., \& Roeyers, H. (2004). Event-rate manipulation and its effect on arousal modulation and response inhibition in adults with high functioning autism. J.Clin.Exp.Neuropsychol., 26, 74-82.

Rees, G., Frith, C. D., \& Lavie, N. (1997). Modulating irrelevant motion perception by varying attentional load in an unrelated task. Science, 278, 1616-1619.

Rees, G., Russell, C., Frith, C. D., \& Driver, J. (1999). Inattentional blindness versus inattentional amnesia for fixated but ignored words. Science, 286, 2504-2507.

Reiss, A. L., Feinstein, C., \& Rosenbaum, K. N. (1986). Autism and genetic disorders. Schizophr.Bull., 12, 724-738.

Riby, D. M. \& Hancock, P. J. (2008). Viewing it differently: social scene perception in Williams syndrome and autism. Neuropsychologia, 46, 2855-2860.

Riggs, K. J., McTaggart, J., Simpson, A., \& Freeman, R. P. (2006). Changes in the capacity of visual working memory in 5- to 10-year-olds. J.Exp.Child Psychol., 95, 18-26.

Rimland, B. (1964). Infantile Autism. New York: Appleton-Century-Crofts.

Rincover, A. \& Ducharme, J. M. (1987). Variables influencing stimulus overselectivity and "tunnel vision" in developmentally delayed children. Am.J.Ment.Defic., 91, 422-430.

Rinehart, N. J. (2001). A deficit in shifting attention present in highfunctioning autism but not Asperger's disorder. Autism, 5, 67-80.

Ro, T., Russell, C., \& Lavie, N. (2001). Changing faces: a detection advantage in the flicker paradigm. Psychol.Sci., 12, 94-99.

Rock, I. \& Gutman, D. (1981). The effect of inattention on form perception. J.Exp.Psychol.Hum.Percept.Perform., 7, 275-285. 
Ronald, A., Happe, F., Bolton, P., Butcher, L. M., Price, T. S., Wheelwright, S. et al. (2006a). Genetic heterogeneity between the three components of the autism spectrum: a twin study. J.Am.Acad.Child Adolesc.Psychiatry, 45, 691-699.

Ronald, A., Happe, F., \& Plomin, R. (2005). The genetic relationship between individual differences in social and nonsocial behaviours characteristic of autism. Dev.Sci., 8, 444-458.

Ronald, A., Happe, F., Price, T. S., Baron-Cohen, S., \& Plomin, R. (2006b). Phenotypic and genetic overlap between autistic traits at the extremes of the general population. J.Am.Acad.Child Adolesc.Psychiatry, 45, 1206-1214.

Rossion, B., Campanella, S., Gomez, C. M., Delinte, A., Debatisse, D., Liard, L. et al. (1999). Task modulation of brain activity related to familiar and unfamiliar face processing: an ERP study. Clin.Neurophysiol., 110, 449-462.

Rouder, J. N., Morey, R. D., Cowan, N., Zwilling, C. E., Morey, C. C., \& Pratte, M. S. (2008). An assessment of fixed-capacity models of visual working memory. Proc.Natl.Acad.Sci.U.S.A, 105, 5975-5979.

Rouse, H., Donnelly, N., Hadwin, J. A., \& Brown, T. (2004). Do children with autism perceive second-order relational features? The case of the Thatcher illusion. J.Child Psychol.Psychiatry, 45, 1246-1257.

Russell, J. (1997). Autism as an Executive Disorder. Oxford: Oxford University Press.

Russell, J., Jarrold, C., \& Henry, L. (1996). Working memory in children with autism and with moderate learning difficulties. J.Child Psychol.Psychiatry, 37, 673-686.

Rutter, M. (1968). Infantile psychosis. Br.J.Psychiatry, 114, 648-649.

Salmond, C. H., Ashburner, J., Connelly, A., Friston, K. J., Gadian, D. G., \& Vargha-Khadem, F. (2005). The role of the medial temporal lobe in autistic spectrum disorders. Eur.J.Neurosci., 22, 764-772.

Sasson, N. J. (2006). The development of face processing in autism. J.Autism Dev.Disord., 36, 381-394.

Saxe, R., Carey, S., \& Kanwisher, N. (2004). Understanding other minds: linking developmental psychology and functional neuroimaging. Annu.Rev.Psychol., $55,87-124$.

Scherer, S. W., Cheung, J., MacDonald, J. R., Osborne, L. R., Nakabayashi, K., Herbrick, J. A. et al. (2003). Human chromosome 7: DNA sequence and biology. Science, 300, 767-772.

Schriebman, L. \& Lovaas, O. I. (1973). Overselective response to social stimuli by autistic children. J.Abnorm.Child Psychol., 1, 152-168. 
Schroer, R. J., Phelan, M. C., Michaelis, R. C., Crawford, E. C., Skinner, S. A., Cuccaro, M. et al. (1998). Autism and maternally derived aberrations of chromosome 15q. Am.J.Med.Genet., 76, 327-336.

Schultz, R. T. (2005). Developmental deficits in social perception in autism: the role of the amygdala and fusiform face area. Int.J.Dev.Neurosci., 23, 125-141.

Schultz, R. T., Gauthier, I., Klin, A., Fulbright, R. K., Anderson, A. W., Volkmar, F. et al. (2000). Abnormal ventral temporal cortical activity during face discrimination among individuals with autism and Asperger syndrome.

Arch.Gen.Psychiatry, 57, 331-340.

Schwarzer, G. (2000). Development of face processing: The effect of face inversion. Child Dev., 71, 391-401.

Senju, A., Tojo, Y., Yaguchi, K., \& Hasegawa, T. (2005). Deviant gaze processing in children with autism: an ERP study. Neuropsychologia, 43, 1297-1306.

Sergent, J. \& Signoret, J. L. (1992). Varieties of functional deficits in prosopagnosia. Cereb.Cortex, 2, 375-388.

Shah, A. \& Frith, U. (1983). An islet of ability in autistic children: a research note. J.Child Psychol.Psychiatry, 24, 613-620.

Shah, A. \& Frith, U. (1993). Why do autistic individuals show superior performance on the block design task? J.Child Psychol.Psychiatry, 34, 1351-1364.

Shallice, T. (1982). Specific impairments of planning. Philos.Trans.R.Soc.Lond B Biol.Sci., 298, 199-209.

Shao, Y., Wolpert, C. M., Raiford, K. L., Menold, M. M., Donnelly, S. L., Ravan, S. A. et al. (2002). Genomic screen and follow-up analysis for autistic disorder. Am.J.Med.Genet., 114, 99-105.

Siegel, B. V., Jr., Asarnow, R., Tanguay, P., Call, J. D., Abel, L., Ho, A. et al. (1992). Regional cerebral glucose metabolism and attention in adults with a history of childhood autism. J.Neuropsychiatry Clin.Neurosci., 4, 406-414.

Smith, H. \& Milne, E. (2009). Reduced change blindness suggests enhanced attention to detail in individuals with autism. J.Child Psychol.Psychiatry, 50, 300306.

Southgate, V., Senju, A., \& Csibra, G. (2007). Action anticipation through attribution of false belief by 2-year-olds. Psychol.Sci., 18, 587-592.

Speer, L. L., Cook, A. E., McMahon, W. M., \& Clark, E. (2007). Face processing in children with autism: effects of stimulus contents and type. Autism, 11, 265-277.

Sperling, G. (1960). The information available in brief visual presentations. Psychological Monograohs, 74, 1-29. 
Spezio, M. L., Adolphs, R., Hurley, R. S., \& Piven, J. (2007). Analysis of face gaze in autism using "Bubbles". Neuropsychologia, 45, 144-151.

Steffenburg, S. (1990). Neurobiological correlates of autism. M.D. University of Göteborg.

Steffenburg, S., Gillberg, C., Hellgren, L., Andersson, L., Gillberg, I. C., Jakobsson, G. et al. (1989). A twin study of autism in Denmark, Finland, Iceland, Norway and Sweden. J.Child Psychol.Psychiatry, 30, 405-416.

Stroop, J. R. (1935). Studies of interference in serial verbal reactions. J.Exp.Psychol., 18, 643-662.

Swettenham, J., Baron-Cohen, S., Charman, T., Cox, A., Baird, G., Drew, A. et al. (1998). The frequency and distribution of spontaneous attention shifts between social and nonsocial stimuli in autistic, typically developing, and nonautistic developmentally delayed infants. J.Child Psychol.Psychiatry, 39, 747-753.

Tager-Flusberg, H. (1981). On the nature of linguistic functioning in early infantile autism. J.Autism Dev.Disord., 11, 45-56.

Tager-Flusberg, H. (1985). Basic level and superordinate level categorization by autistic, mentally retarded, and normal children. J.Exp.Child Psychol., 40, 450469.

Tager-Flusberg, H. (1992). Autistic children's talk about psychological states: deficits in the early acquisition of a theory of mind. Child Dev., 63, 161-172.

Tager-Flusberg, H. (2003). Exploring the relationships between theory of mind and social-communicative functioning in children with autism. In B.Repacholi $\&$ V. Slaughter (Eds.), Individual differences in theory of mind: Implications for typical and atypical development (pp. 197-212). London: Psychology Press.

Talay-Ongan, A. \& Wood, K. (2000). Unusual sensory sensitivities in autism: a possible crossroads. International Journal of Disability, Development and Education, 47, 201-212.

Tanaka, J., Kay, J., Grinnell, E., Stanfield, B., \& Szechter, L. (1998). Face recognition in young children: When the whole is greater than the sum of its parts. Visual Cognition, 5, 479-496.

Tantam, D., Monaghan, L., Nicholson, H., \& Stirling, J. (1989). Autistic children's ability to interpret faces: a research note. J.Child Psychol.Psychiatry, 30, 623-630.

Tarr, M. J. \& Gauthier, I. (2000). FFA: a flexible fusiform area for subordinate-level visual processing automatized by expertise. Nat.Neurosci., 3, 764769 .

Teder-Salejarvi, W., Pierce, K., Courchesne, E., \& Hillyard, S. (2005). Auditory spatial localization and attentional deficits in adults with autism. Cognitive Brain Research, 23, 221-234. 
Teunisse, J. P., Cools, A. R., van Spaendonck, K. P., Aerts, F. H., \& Berger, H. J. (2001). Cognitive styles in high-functioning adolescents with autistic disorder. J.Autism Dev.Disord., 31, 55-66.

Teunisse, J. P. \& de Gelder, B. (2003). Face processing in adolescents with autistic disorder: the inversion and composite effects. Brain Cogn, 52, 285-294.

Theeuwes, J., Kramer, A. F., \& Belopolsky, A. V. (2004). Attentional set interacts with perceptual load in visual search. Psychon.Bull.Rev., 11, 697-702.

Theeuwes, J. \& Van der Stigchel, S. (2006). Faces capture attention: Evidence from inhibition of return. Visual Cognition, 13, 657-665.

Thompson, P. (1980). Margaret Thatcher: a new illusion. Perception, 9, 483484

Tipper, S. P. (1985). The negative priming effect: inhibitory effects of ignored primes. Quarterly Journal of Experimental Psychology, 37A, 571-590.

Todd, J. J. \& Marois, R. (2004). Capacity limit of visual short-term memory in human posterior parietal cortex. Nature, 428, 751-754.

Tong, F. \& Nakayama, K. (1999). Robust representations for faces: evidence from visual search. J.Exp.Psychol.Hum.Percept.Perform., 25, 1016-1035.

Tong, F., Nakayama, K., Moscovitch, M., Weinrib, O., \& Kanwisher, N. (2000). Response properties of the human fusiform face area. Cognit.Neuropsychol., 17, 257-279.

Townsend, J. \& Courchesne, E. (1994). Parietal damage and narrow 'spotlight' spatial attention. J.Cogn Neurosci., 6, 220-232.

Townsend, J., Courchesne, E., \& Egaas, B. (1996a). Slowed orienting of covert visual-spatial attention in autism: Specific deficits associated with cerebellar and parietal abnormality. Development and Psychopathology, 8, 563-584.

Townsend, J., Singer-Harris, N., \& Courchesne, E. (1996b). Visual attention abnormalities in autism: Delayed orienting to a location. Journal of the International Neuropsychological Society, 2, 1-10.

Treisman, A. M. (1964). Selective attention in man. British Medical Bulletin, 20, 12-16.

Treisman, A. M. (1969). Strategies and models of selective attention. Psychol.Rev., 76, 282-299.

Treisman, A. M. \& Gelade, G. (1980). A feature-integration theory of attention. Cognit.Psychol., 12, 97-136.

Turner, M. (1997). Towards an Executive Dysfunction Account of Repetitive Behavior in Autism. In J.Russell (Ed.), (pp. 57-100). Oxford: Oxford University Press. 
Turner, M. (1999a). Annotation: Repetitive behaviour in autism: a review of psychological research. J.Child Psychol.Psychiatry, 40, 839-849.

Turner, M. A. (1999b). Generating novel ideas: fluency performance in highfunctioning and learning disabled individuals with autism. J.Child

Psychol.Psychiatry, 40, 189-201.

Valenza, E., Simion, F., Cassia, V. M., \& Umilta, C. (1996). Face preference at birth. J.Exp.Psychol.Hum.Percept.Perform., 22, 892-903.

Verte, S., Geurts, H. M., Roeyers, H., Oosterlaan, J., \& Sergeant, J. A. (2005). Executive functioning in children with autism and Tourette syndrome. Dev.Psychopathol., 17, 415-445.

Vignal, J. P., Chauvel, P., \& Halgren, E. (2000). Localized face-processing by the human prefrontal cortex: Stimulation-evoked hallucinations of faces.

Cognit.Neuropsychol., 17, 281-291.

Vogel, E. K., Woodman, G. F., \& Luck, S. J. (2001). Storage of features, conjunctions and objects in visual working memory.

J.Exp.Psychol.Hum.Percept.Perform., 27, 92-114.

Von Wright, J. M. (1970). On selection in visual immediate memory. In A.F.Sanders (Ed.), Attention and Performance (pp. 280-292). Amsterdam: North Holland.

Wainwright, J. A. \& Bryson, S. E. (1996). Visual-spatial orienting in autism. J.Autism Dev.Disord., 26, 423-438.

Wainwright-Sharp, J. A. \& Bryson, S. E. (1993). Visual orienting deficits in high-functioning people with autism. J.Autism Dev.Disord., 23, 1-13.

Webb, S., Nalty, T., Munson, J., Brock, C., Abbott, R., \& Dawson, G. (2007). Rate of head circumference growth as a function of autism diagnosis and history of autistic regression. J.Child Neurol., 22, 1182-1190.

Wechsler, D. (1999). Wechsler Abbreviated Scale for Intelligence. San Antonio, TX: Psychological Corporation.

Wicker, B., Michel, F., Henaff, M. A., \& Decety, J. (1998). Brain regions involved in the perception of gaze: a PET study. Neuroimage., 8, 221-227.

Williams, D. (1992). Nobody Nowhere: The Extraordinary Autobiography of an Autistic. New York: Random House.

Williams, J. H., Waiter, G. D., Gilchrist, A., Perrett, D. I., Murray, A. D., \& Whiten, A. (2006). Neural mechanisms of imitation and 'mirror neuron' functioning in autistic spectrum disorder. Neuropsychologia, 44, 610-621.

Williams, J. H., Whiten, A., Suddendorf, T., \& Perrett, D. I. (2001). Imitation, mirror neurons and autism. Neurosci.Biobehav.Rev., 25, 287-295. 
Wimmer, H. \& Perner, J. (1983). Beliefs about beliefs: representation and constraining function of wrong beliefs in young children's understanding of deception. Cognition, 13, 103-128.

Wing, L. \& Gould, J. (1979). Asperger Syndrome: a clinical account. Journal of Autism and Developmental Disorders, 9, 11-29.

Winston, J. S., Strange, B. A., O'Doherty, J., \& Dolan, R. J. (2002). Automatic and intentional brain responses during evaluation of trustworthiness of faces. Nat.Neurosci., 5, 277-283.

Witkin, H. A., Oltman, P. K., Raskin, E., \& Karp, S. (1971). A Manual for the Embedded Figures Test. California: Consulting Psychology Press.

Wojciulik, E., Kanwisher, N., \& Driver, J. (1998). Covert visual attention modulates face-specific activity in the human fusiform gyrus: fMRI study. J.Neurophysiol., 79, 1574-1578.

Wulff, S. B. (1985). The symbolic and object play of children with autism: a review. J.Autism Dev.Disord., 15, 139-148.

Yin, R. K. (1969). Looking at upside-down faces. J.Exp.Psychol., 81, 141145.

Young, A. W., Ellis, A. W., Flude, B. M., McWeeny, K. H., \& Hay, D. C. (1986a). Face-name interference. J.Exp.Psychol.Hum.Percept.Perform., 12, 466-475.

Young, A. W., Hellawell, D., \& Hay, D. C. (1987). Configurational information in face perception. Perception, 16, 747-759.

Young, A. W., McWeeny, K. H., Hay, D. C., \& Ellis, A. W. (1986b). Matching familiar and unfamiliar faces on identity and expression. Psychol.Res., 48, 63-68.

Zilbovicius, M., Garreau, B., Samson, Y., Remy, P., Barthelemy, C., Syrota, A. et al. (1995). Delayed maturation of the frontal-cortex in childhood autism. American Journal of Psychiatry, 152, 248-252. 